\title{
A Structure Set in Stone: Designing Rigid Linkers to Control the Efficiency of Intramolecular Singlet Fission
}

Lukas Ahrens, ${ }^{1, \#}$ Nikolaus Wollscheid, ${ }^{2,4, \#}$ Jie Han, ${ }^{3,4, \S}$ Oskar Kefer, ${ }^{2,4}$ Frank Rominger, ${ }^{1}$ Ashkan Roozbeh, ${ }^{2,4}$ Jan Freudenberg ${ }^{1, *}$, Andreas Dreuw ${ }^{3,4^{*}}$, Uwe H. F. Bunz,,$^{2,4, *}$ Tiago Buckup ${ }^{2,4^{*}}$

${ }^{1}$ Organisch-Chemisches Institut, Ruprecht-Karls-Universität, D-69120 Heidelberg, Germany.

${ }^{2}$ Physikalisch Chemisches Institut, Ruprecht-Karls-Universität, D-69120 Heidelberg, Germany.

${ }^{3}$ Interdisziplinäres Zentrum für Wissenschaftliches Rechnen, Ruprecht-Karls-Universität Heidelberg, D69120, Heidelberg, Germany

${ }^{4}$ Centre for Advanced Materials, Ruprecht-Karls-Universität, D-69120 Heidelberg, Germany.

§Present Address: State Key Laboratory of Coordination Chemistry, Jiangsu Key Laboratory of Advanced Organic Materials, Chemistry and Biomedicine Innovation Center (ChemBIC), School of Chemistry and Chemical Engineering, Nanjing University, Nanjing 210023, China.

\# These authors contributed equally to this work.

*Corresponding Authors:

tiago.buckup@pci.uni-heidelberg.de, uwe.bunz@oci.uni-heidelberg.de, dreuw@uni-heidelberg.de, freudenberg@oci.uni-heidelberg.de 


\section{Contents}

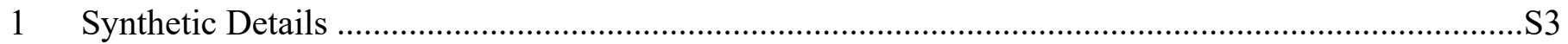

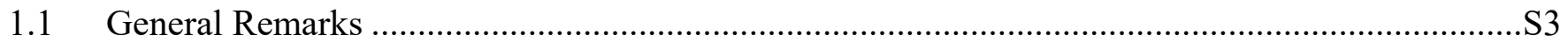

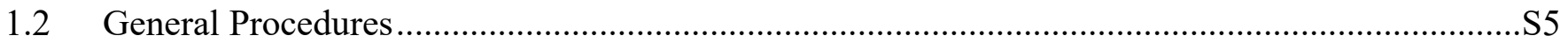

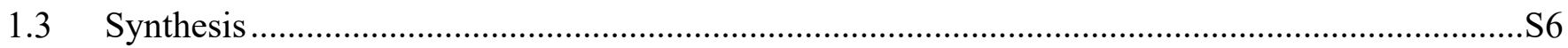

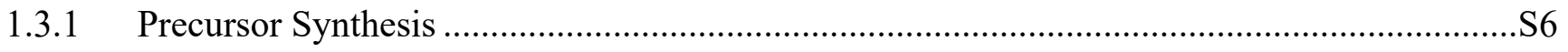

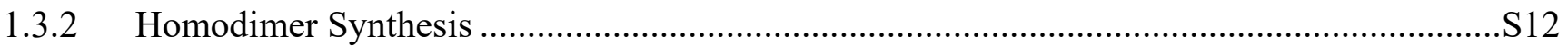

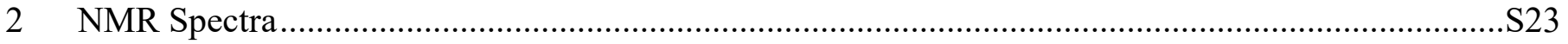

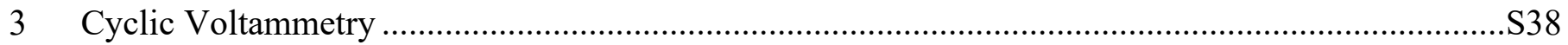

4 Crystal Structures and Optimized Geometries .....................................................................................

5 Comparison of Experimental and Calculated Spectra.........................................................................

5.1 Comparison of Steady-State Absorption Spectra and Calculated Ground-State Transitions.........S49

5.2 Comparison of SADS and Calculated Excited-State Transitions...................................................S50

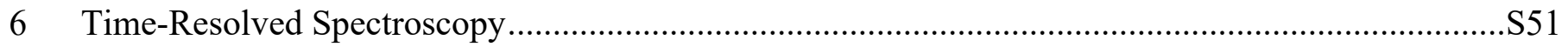

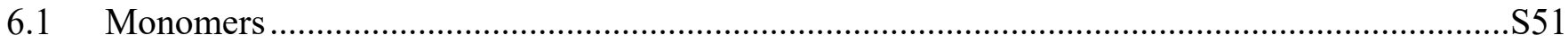

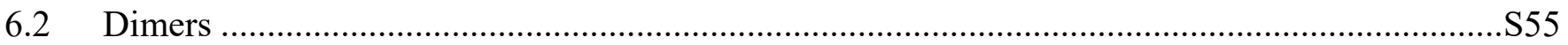

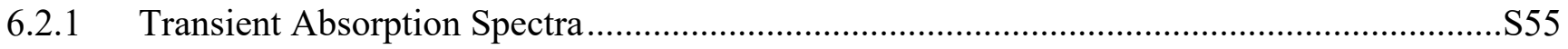

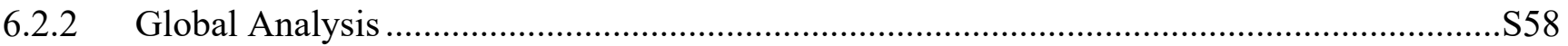

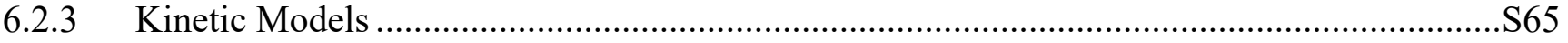

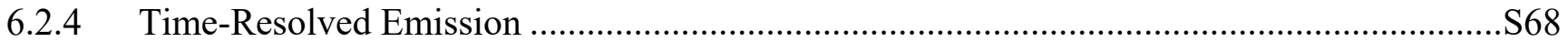

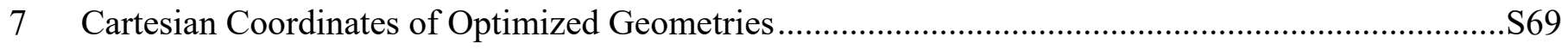

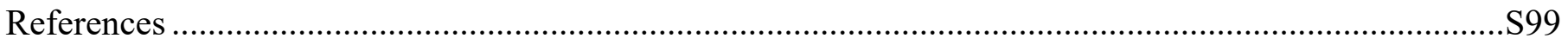




\section{Synthetic Details}

\subsection{General Remarks}

\section{Reagents and solvents for synthesis}

All reagents were obtained from commercial suppliers (ABCR, ACROS, ALFA AESAR, MERCK and TCI) and were used without further purification if not otherwise stated. Anhydrous solvents were dispensed from the solvent purification system MBRAUN MB SPS 800. Deuterated solvents were bought from MERCK (Darmstadt, Germany). All reactions requiring exclusion of oxygen and moisture were carried out in heatgun dried glassware under a dry and oxygen free nitrogen or argon atmosphere using Schlenk techniques.

\section{Thin layer chromatography (TLC)}

$R_{\mathrm{f}-\mathrm{values}}$ were determined by polyester sheets coated with silica gel produced by MACHERY-NAGEL (Polygram ${ }^{\circledR}$ SIL G/UV 254 ). To visualize the substances, the TLC-plate was exposed to ultraviolet light (254 and $365 \mathrm{~nm})$.

\section{Column chromatography}

Column chromatography was performed using silica gel from MACHEREY, NAGEL \& Co. KG (Düren, Germany) (particle size: $0.040-0.063 \mathrm{~mm}$ ) as stationary phase. Before column chromatography the crude product was mixed with Celite 545 and dichloromethane to make a slurry. The solvent was removed by rotary evaporation to get a dry powder.

\section{Gel permeation chromatography (GPC)}

Preparative GPC was performed on Bio-Beads ${ }^{\circledR}$ (S-X1 Beads, 200 - 400 Mesh, crosslinked polystyrene), purchased from BIO-RAD LABORATORIES, Inc., using toluene as eluent.

\section{${ }^{1} \mathrm{H}$ NMR spectra and ${ }^{13} \mathrm{C}$ NMR spectra}

All spectra were recorded at room temperature at the chemistry department of the University of Heidelberg under the direction of Dr. J. Graf on a BRUKER Avance III $600\left({ }^{1} \mathrm{H}: 600 \mathrm{MHz},{ }^{13} \mathrm{C}: 151 \mathrm{MHz}\right)$, BRUKER Avance III $400\left({ }^{1} \mathrm{H}: 400 \mathrm{MHz},{ }^{13} \mathrm{C}: 101 \mathrm{MHz}\right)$ or BRUKER Avance III $300\left({ }^{1} \mathrm{H}: 301 \mathrm{MHz}\right) .{ }^{13} \mathrm{C}$ NMR spectra were measured proton decoupled. NMR spectra were integrated and processed using the Software TopSpin 3.5pl5 (BRUKER). For calibration the residual solvent peaks were referenced ( $\mathrm{CDCl}_{3}: 7.26 \mathrm{ppm} / 77.16 \mathrm{ppm}$; 
$\mathrm{CD}_{2} \mathrm{Cl}_{2}$ : $5.32 \mathrm{ppm} / 53.84$ ppm; DMSO-d 6 : $2.50 \mathrm{ppm} / 39.52 \mathrm{ppm}$, THF-d8: $\left.1.72 \mathrm{ppm} / 25.31 \mathrm{ppm}\right){ }^{1}$ Chemical shifts $\delta$ are reported in ppm and coupling constants $J$ in Hz. The following abbreviations describe the observed multiplicities: $\mathrm{s}=$ singlet, $\mathrm{bs}=$ broad singlet, $\mathrm{d}=$ doublet, $\mathrm{t}=$ triplet, $\mathrm{m}=$ multiplet.

\section{IR spectra}

All spectra were recorded neat at room temperature on a JASCO FT/IR-4100 LE. The most important signals are reported in wavenumbers $\left(\mathrm{cm}^{-1}\right)$.

\section{Mass spectrometry}

Mass spectra were recorded at the chemical department of the University of Heidelberg under the direction of Dr. J. Gross using the following instruments: JEOL JMS 700 (EI), BRUKER ApexQe hybrid 9.4 T FT-ICR (DART, ESI, MALDI); BRUKER AutoFlex Speed time-of-flight (DART, ESI, MALDI).

\section{Melting points}

Melting points were determined in open glass capillaries with a Melting Point Apparatus MEL-TEMP (Electrothermal, Rochford, UK).

\section{X-ray single-crystal structure analysis}

X-ray single-crystal structure analyses were measured on a BRUKER Smart APEX-II Quazar Area Detector diffractometer or a STOE Stadivari diffractometer under the direction of Dr. F. Rominger (Heidelberg University). Diffraction intensities were corrected for Lorentz and polarization effects. An empirical absorption correction was applied using SADABS based on the Laue symmetry of reciprocal space. Heavy atom diffractions were solved by direct methods and refined against $\mathrm{F}^{2}$ with the full matrix least square algorithm. Hydrogen atoms were either isotropically refined or calculated. The structures were solved and refined using the SHELXTL software package.

\section{IUPAC names and formulas}

All mentioned IUPAC names were determined by ACD/ChemSketch 2012 of ADVANCED CHEMISTRY DeVelopment Inc. and all formulas drawn by ChemDraw Professional 16.0. 


\subsection{General Procedures}

GP1: Oxidation of biscatechols with sodium periodate

In a flask was dissolved biscatechol (1.00 equiv.) in ethanol and was then diluted with dichloromethane. A solution of sodium periodate (2.20 equiv.) in distilled water was added and the reaction mixture stirred at room temperature for $1 \mathrm{~h}$. The phases were separated, and the aqueous layer was extracted with dichloromethane. The combined organic phases were washed with brine, dried over magnesium sulfate and filtrated. The solvent was removed under reduced pressure and the oxidation product was isolated.

GP2: Condensation of bis-ortho-quinones with ortho-diamines

In a heatgun dried Schlenk tube under an atmosphere of argon was added the bis-ortho-quinone (1.00 equiv.) and the ortho-diamine (2.00 equiv.). Then chloroform and acetic acid were added and the reaction mixture stirred at $50^{\circ} \mathrm{C}$ for $15 \mathrm{~h}$. The mixture was cooled to room temperature and diluted with water $(10 \mathrm{~mL})$. The phases were separated, and the aqueous layer was extracted with dichloromethane $(3 \times 10 \mathrm{~mL})$. The combined organic phases were washed with sodium bicarbonate solution $(10 \mathrm{~mL})$, dried over magnesium sulfate and filtrated. The solvent was removed under reduced pressure and the crude product was absorbed on Celite. After flash column chromatography (petroleum ether/diethyl ether) and gel permeation chromatography (toluene) the condensation product was isolated.

GP3: In situ oxidation of biscatechols and condensation with ortho-diamines

In a flask was dissolved either the biscatechol (1.00 equiv.) in ethanol and was then diluted with dichloromethane. A solution of either sodium periodate (2.20 equiv.) in distilled water was added and the reaction mixture stirred at room temperature for either $1 \mathrm{~h}$. The phases were separated, and the aqueous layer was extracted with dichloromethane (as little as possible). The product was used without further purification.

In a heatgun dried Schlenk tube under an atmosphere of argon was added the ortho-diamine (2.00 equiv.). Then in situ prepared ortho-quinone in dichloromethane was added, followed by acetic acid ( $1: 1 \mathrm{v} / \mathrm{v})$ and the

reaction mixture stirred at $50{ }^{\circ} \mathrm{C}$ for $15 \mathrm{~h}$. The mixture was cooled to room temperature and diluted with water $(10 \mathrm{~mL})$. The phases were separated, and the aqueous layer was extracted with dichloromethane $(3 \times 10 \mathrm{~mL})$. The combined organic phases were washed with sodium bicarbonate solution $(10 \mathrm{~mL}), \mathrm{dried}$ over magnesium sulfate and filtrated. The solvent was removed under reduced pressure and the crude product 
was absorbed on Celite. After flash column chromatography (petroleum ether/diethyl ether) and gel permeation chromatography (toluene) the condensation product was isolated.

\subsection{Synthesis}

3,3,3',3',7,7'-Hexamethyl-2,2',3,3'-tetrahydro-1,1'-spirobi[indene]-5,5',6,6'-tetrol (S1) ${ }^{2} \quad\{[$ 7,7'-bis(bromomethyl)-3,3,3',3'-tetramethyl-2,2',3,3'-tetrahydro-1,1'-spirobi[indene]-5,5',6,6'-tetrayl]tetrakis(oxy)\} tetrakis[tert-butyl(dimethyl)silane] (S3), ${ }^{2}$ [(10,10,13,13-tetramethyl-10,11,12,13-tetrahydro-4H,6H-diindeno[7,1$c d: 1^{\prime}, 7^{\prime}$-ef] oxocine-2,3,7,8-tetrayl)tetrakis(oxy)]tetrakis[tert-butyl(dimethyl)silane] (S4), ${ }^{2} \quad$ 1,4-bis((triisopropylsilyl)ethynyl)anthracene-2,3-diamine (7a), ${ }^{3}$ 1,4-bis((triisopropylsilyl)ethynyl)phenazine-2,3-diamine (7b) ${ }^{3} \quad$ 4,7-bis((triisopropylsilyl)ethynyl)benzo[c][1,2,5]thiadiazole-5,6-diamine $\quad(\mathbf{7 c}),{ }^{4} \quad$ 6,13-bis((triisopropylsilyl)ethynyl)naphtho[2,3-b]phenazine (8a) ${ }^{5}$ and 6,13-bis((triisopropylsilyl)ethynyl)quinoxalino[2,3-b]phenazine (8b) $)^{6}$ and 4,11-bis((triisopropylsilyl)ethynyl)-[1,2,5] thiadiazolo[3,4-b]phenazine $(\mathbf{8 c})^{7}$ were synthesized according to literature procedures.

\subsubsection{Precursor Synthesis}

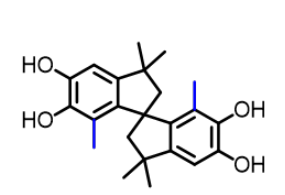

s1
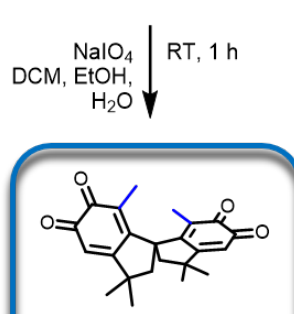

$4,96 \%$

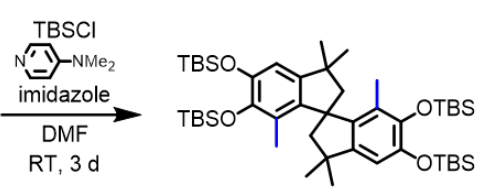

TBS $=1-\mathrm{Si}_{1}^{\mathrm{i}} \mathrm{t}$

S2, $77 \%$
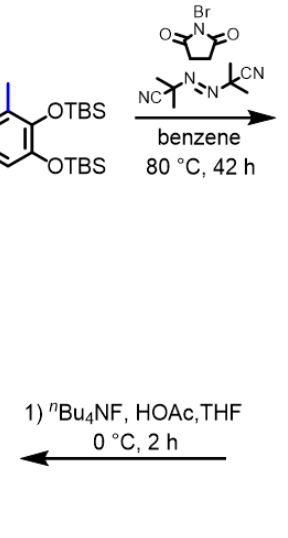

S5, $99 \%$

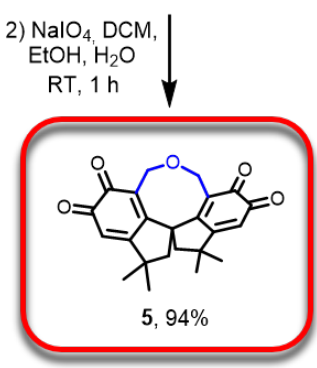

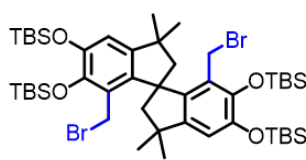

S3, $92 \%$
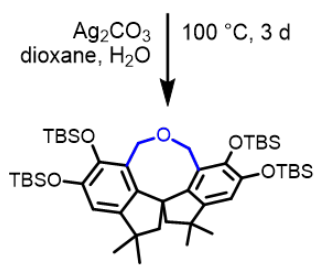

S4, $41 \%$
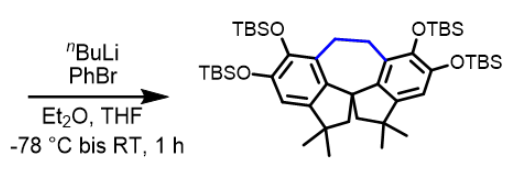

S6, 93\%

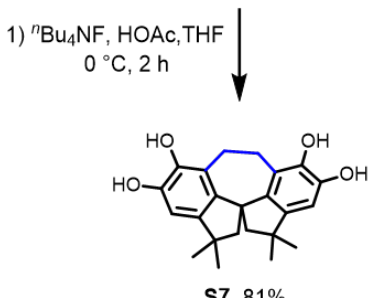

s7, $81 \%$

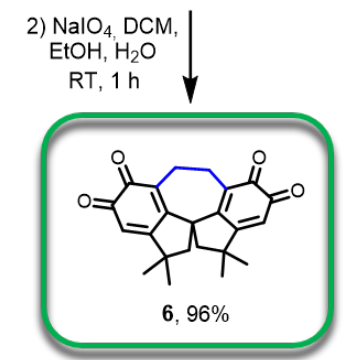

Scheme S1: Synthesis of spiro-linked ortho-quinones 4 - 6. 


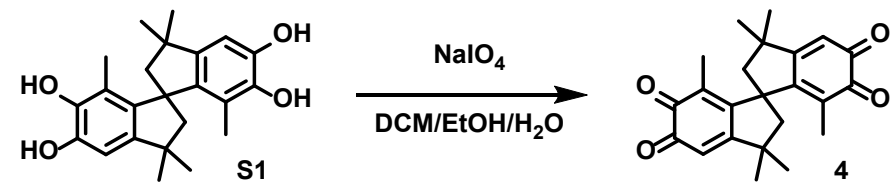

GP1 was applied to racemic 3,3,3',3',7,7'-hexamethyl-2,2',3,3'-tetrahydro-1,1'-spirobi[1H-indene]-5,5',6,6'tetrol (S1) (2.00 g, $8.81 \mathrm{mmol}, 1.00$ equiv.) and sodium periodate ( $2.55 \mathrm{~g}, 11.9 \mathrm{mmol}, 2.20$ equiv.) in $30 \mathrm{~mL}$ ethanol, $100 \mathrm{~mL}$ dichloromethane and $30 \mathrm{~mL}$ distilled water. After workup, racemic bis-ortho-quinone $\mathbf{4}$ was isolated as a red solid $(1.89 \mathrm{~g}, 5.20 \mathrm{mmol}, 96 \%)$.

$\mathbf{R}_{f}=0.20\left(\mathrm{SiO}_{2} ;\right.$ petroleum ether/ethyl acetate $\left.2: 1, \mathrm{v} / \mathrm{v}\right)$.

Mp: $\geq 211^{\circ} \mathrm{C}$ (decomposition).

${ }^{1} \mathbf{H}$ NMR $\left(\mathrm{CDCl}_{3}, 300 \mathrm{MHz}, \mathrm{rt}\right): \delta=6.23(\mathrm{~s}, 2 \mathrm{H}), 2.22-2.38(\mathrm{~m}, 4 \mathrm{H}), 1.78(\mathrm{~s}, 6 \mathrm{H}), 1.41(\mathrm{~s}, 6 \mathrm{H}), 1.39(\mathrm{~s}, 6 \mathrm{H})$ ppm.

${ }^{13} \mathbf{C}\left\{{ }^{1} \mathbf{H}\right\}$ NMR $\left(\mathrm{CDCl}_{3}, 101 \mathrm{MHz}, \mathrm{rt}\right): \delta=179.5,178.7,169.8,158.1,133.6,120.3,54.0,43.5,31.5,28.0$, $12.9 \mathrm{ppm}$.

IR (ATR): $\tilde{\boldsymbol{v}}=2965,2929,2868,1646,1587,1335,1287,1031,671,423 \mathrm{~cm}^{-1}$.

HRMS (EI $) m / z:[\mathrm{M}]^{+}$: calcd. for $\left[\mathrm{C}_{23} \mathrm{H}_{24} \mathrm{O}_{4}\right]^{+}: 364.1669$; found 364.1659; correct isotope distribution.

10,10,13,13-Tetramethyl-10,11,12,13-tetrahydro-4H,6H-diindeno[7,1-cd:1',7'-ef] oxocine-2,3,7,8-tetrol (S5)

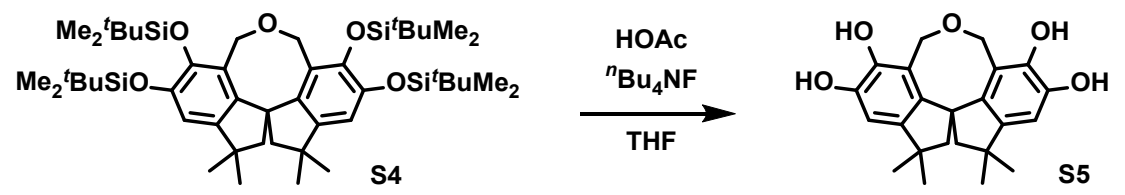

In a Schlenk tube under an atmosphere of argon was dissolved S4 (420 mg, $500 \mu \mathrm{mol}, 1.00$ equiv.) in $4.00 \mathrm{~mL}$ anhydrous tetrahydrofuran. Acetic acid $(120 \mu \mathrm{L}, 126 \mathrm{mg}, 2.10 \mathrm{mmol}, 4.20$ equiv.) and thereafter tetra- $n$-butylammonium fluoride ( $1.0 \mathrm{~mol} \mathrm{~L}^{-1}$ in tetrahydrofuran, $2.10 \mathrm{~mL}, 549 \mathrm{mg}, 2.10 \mathrm{mmol}, 4.20$ equiv.) were added dropwise at $0{ }^{\circ} \mathrm{C}$ and the reaction mixture was stirred at $0{ }^{\circ} \mathrm{C}$ for $2 \mathrm{~h}$. The solvent was removed 
under reduced pressure, the crude product was dissolved in $15 \mathrm{~mL}$ ethyl acetate and washed with water $(5 \times 10 \mathrm{~mL})$. The organic phases were dried over magnesium sulfate and filtrated. The solvent was removed under reduced pressure and the crude product was absorbed on Celite. After flash column chromatography (dichloromethane/methanol 50:1 v/v -> 30:1) the product S5 was isolated as a colorless solid (190 mg, $498 \mu \mathrm{mol}, 99 \%)$.

$\mathbf{R}_{\boldsymbol{f}}=0.41\left(\mathrm{SiO}_{2} ;\right.$ dichloromethane/methanol 10:1 v/v).

Mp: $\geq 260{ }^{\circ} \mathrm{C}$ (decomposition).

${ }^{1}$ H NMR (DMSO-d6, $\left.300 \mathrm{MHz}, \mathrm{rt}\right): \delta=9.04(\mathrm{~s}, 2 \mathrm{H}), 8.03(\mathrm{~s}, 2 \mathrm{H}), 6.55(\mathrm{~s}, 2 \mathrm{H}), 4.52$ (d, J=11.5 Hz, 2H), $3.95(\mathrm{~d}, J=11.5 \mathrm{~Hz}, 2 \mathrm{H}), 2.28(\mathrm{~d}, J=12.5 \mathrm{~Hz}, 2 \mathrm{H}), 1.74(\mathrm{~d}, J=12.5 \mathrm{~Hz}, 2 \mathrm{H}), 1.37(\mathrm{~s}, 6 \mathrm{H}), 1.15(\mathrm{~s}, 6 \mathrm{H})$ ppm.

${ }^{13} \mathbf{C}\left\{{ }^{1} \mathbf{H}\right\}$ NMR (DMSO-d6, $\left.101 \mathrm{MHz}, \mathrm{rt}\right): \delta=145.1,142.8,141.1,140.2,116.1,108.9,57.5,57.2,55.4,41.1$, $32.3,30.0 \mathrm{ppm}$.

IR (ATR): $\tilde{\boldsymbol{v}}=3330,2955,2866,1600,1456,1294,1235,1203,988,856 \mathrm{~cm}^{-1}$.

HRMS (DART-) $m / z:[\mathrm{M}-\mathrm{H}]^{-}:$calcd. for $\left[\mathrm{C}_{23} \mathrm{H}_{25} \mathrm{O}_{5}\right]^{-:}$: 381.1707; found 381.1711; correct isotope distribution.

10,10,13,13-Tetramethyl-10,11,12,13-tetrahydro-3H,6H-diindeno[7,1-cd:1',7'-ef]oxocine-2,3,-7,8(4H)tetrone (5)
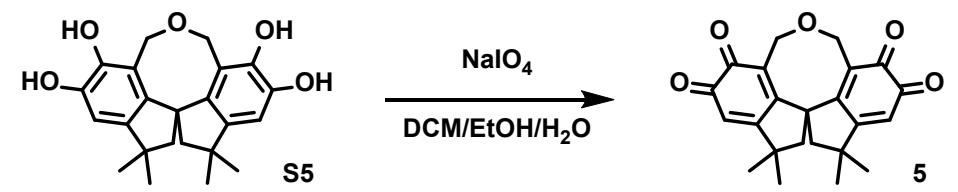

GP1 was applied to racemic biscatechol S5 (190 mg, $497 \mu \mathrm{mol}, 1.00$ equiv.) and sodium periodate (234 mg, 1.09 mmol, 2.20 equiv.) in $1 \mathrm{~mL}$ ethanol, $3 \mathrm{~mL}$ dichloromethane and $4 \mathrm{~mL}$ distilled water. After workup, racemic bis-ortho-quinone 5 was isolated as a red solid (177 $\mathrm{mg}, 468 \mu \mathrm{mol}, 94 \%$ ).

$\mathbf{R}_{\boldsymbol{f}}=0.17\left(\mathrm{SiO}_{2} ;\right.$ petroleum ether/ethyl acetate $\left.2: 1, \mathrm{v} / \mathrm{v}\right)$.

Mp: $\geq 234{ }^{\circ} \mathrm{C}$ (decomposition). 
${ }^{1} \mathbf{H}$ NMR $\left(\mathrm{CDCl}_{3}, 300 \mathrm{MHz}, \mathrm{rt}\right): \delta=6.35(\mathrm{~s}, 2 \mathrm{H}), 4.87(\mathrm{~d}, J=13.0 \mathrm{~Hz}, 2 \mathrm{H}), 4.12(\mathrm{~d}, J=13.0 \mathrm{~Hz}, 2 \mathrm{H}), 2.55$ (d, $J=13.3 \mathrm{~Hz}, 2 \mathrm{H}), 1.98$ (d, $J=13.3 \mathrm{~Hz}, 2 \mathrm{H}), 1.54$ (s, 6H), 1.39 (s, 6H) ppm.

${ }^{13} \mathbf{C}\left\{{ }^{1} \mathbf{H}\right\}$ NMR $\left(\mathrm{CDCl}_{3}, 101 \mathrm{MHz}, \mathrm{rt}\right): \delta=178.4,177.6,167.8,163.2,124.8,123.5,57.3,56.0,53.6,42.2$, $31.5,30.5 \mathrm{ppm}$.

IR (ATR): $\tilde{\boldsymbol{v}}=2961,2923,2871,1656,1652,1362,1334,1235,1208,997,868,661 \mathrm{~cm}^{-1}$.

HRMS $\left(\mathrm{ESI}^{+}\right) m / z:[\mathrm{M}]^{++}$: calcd. for $\left[\mathrm{C}_{23} \mathrm{H}_{22} \mathrm{NaO}_{5}\right]^{++}: 401.1359$; found 401.1369; correct isotope distribution.

\section{Crystal data}

Single crystalline specimen were obtained by slow diffusion of methanol into a chloroform solution of 5:

Orange crystal (plank), dimensions $0.124 \times 0.113 \times 0.023 \mathrm{~mm}^{3}$, crystal system monoclinic, space group $\mathrm{C} 2 / \mathrm{c}, Z=20, a=42.861(2) \AA, b=9.1509(3) \AA, c=25.3723(12) \AA, \alpha=90^{\circ}, \beta=96.835(4)^{\circ}, \gamma=90^{\circ}$, $V=9880.6(7) \AA^{3}, \rho=1.316 \mathrm{~g} / \mathrm{cm}^{3}, T=200(2) \mathrm{K}, \Theta_{\max }=66.591^{\circ}, 40195$ reflections measured, 8667 unique $\left(\mathrm{R}_{\text {int }}=0.0561\right), 5420$ observed $(\mathrm{I}>2 \sigma(\mathrm{I})), \mu=0.78 \mathrm{~mm}^{-1}, T_{\min }=0.72, T_{\max }=1.24,667$ parameters refined, hydrogen atoms were treated using appropriate riding models, goodness of fit 1.02 for observed reflections, final residual values $\mathrm{R} 1(\mathrm{~F})=0.058, \mathrm{wR}\left(\mathrm{F}^{2}\right)=0.136$ for observed reflections, residual electron density -0.68 to $0.88 \mathrm{e}^{-3}$.

$[(2,2,11,11$-Tetramethyl-1,2,6,7,11,12-hexahydrobenzo[cd]indeno[7,1-hi]azulene-4,5,8,9-tetrayl)tetrakis(oxy)]tetrakis[tert-butyl(dimethyl)silane] (S6)
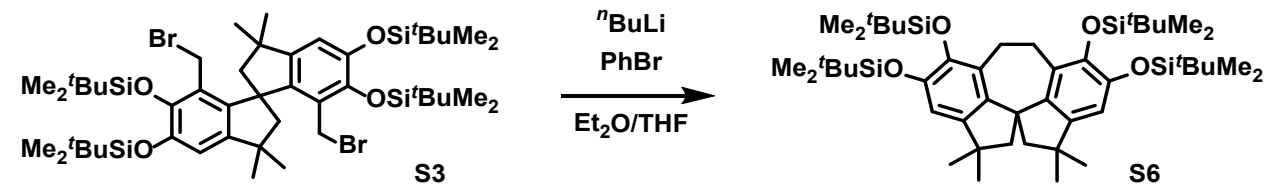

In a heatgun dried Schlenk tube under an atmosphere of argon was dissolved bromobenzene $(639 \mu \mathrm{L}, 958 \mathrm{mg}$, $6.10 \mathrm{mmol}, 1.50$ equiv. $)$ in $8.00 \mathrm{~mL}$ anhydrous diethyl ether. $n$-Butyllithium $\left(2.5 \mathrm{~mol} \mathrm{~L}^{-1}\right.$ in hexanes, $2.44 \mathrm{~mL}, 391 \mathrm{mg}, 6.10 \mathrm{mmol}, 1.50$ equiv.) was added dropwise at $-78{ }^{\circ} \mathrm{C}$, the reaction mixture was stirred at $-78^{\circ} \mathrm{C}$ for $10 \mathrm{~min}$ and thereafter at room temperature for $1 \mathrm{~h}$. In a second heatgun dried Schlenk tube under an atmosphere of argon was dissolved S3 (4.00 g, $4.07 \mathrm{mmol}, 1.00$ equiv.) in $16.0 \mathrm{~mL}$ anhydrous diethyl ether and $16.0 \mathrm{~mL}$ anhydrous tetrahydrofuran and then previously made phenyllithium solution was quickly 
added at room temperature. The resulting reaction mixture was stirred at room temperature for $1.5 \mathrm{~h}$ and then diluted with water $(20 \mathrm{~mL})$. The phases were separated, and the aqueous layer was extracted with dichloromethane $(3 \times 20 \mathrm{~mL})$. The combined organic phases were dried over magnesium sulfate and filtrated. The solvent was removed under reduced pressure and the crude product was absorbed on Celite. After flash column chromatography (petroleum ether/dichloromethane 500:1 v/v -> 250:1 -> 100:1) the product S6 was isolated as a colorless solid (3.12 g, $3.78 \mathrm{mmol}, 93 \%)$.

$\mathbf{R}_{\boldsymbol{f}}=0.25\left(\mathrm{SiO}_{2}\right.$; petroleum ether).

Mp: $142^{\circ} \mathrm{C}$.

${ }^{1} \mathbf{H}$ NMR $\left(\mathrm{CDCl}_{3}, 600 \mathrm{MHz}, \mathrm{rt}\right): \delta=6.67(\mathrm{~s}, 2 \mathrm{H}), 4.17(\mathrm{~d}, J=9.50 \mathrm{~Hz}, 2 \mathrm{H}), 3.86(\mathrm{~d}, J=9.50 \mathrm{~Hz}, 2 \mathrm{H}), 2.71$ $(\mathrm{d}, J=13.8 \mathrm{~Hz}, 2 \mathrm{H}), 2.22(\mathrm{~d}, J=13.8 \mathrm{~Hz}, 2 \mathrm{H}), 1.40(\mathrm{~s}, 6 \mathrm{H}), 1.28(\mathrm{~s}, 6 \mathrm{H}), 1.01(\mathrm{~s}, 18 \mathrm{H}), 0.95(\mathrm{~s}, 18 \mathrm{H}), 0.30$ $(\mathrm{s}, 6 \mathrm{H}), 0.27(\mathrm{~s}, 6 \mathrm{H}), 0.19(\mathrm{~s}, 6 \mathrm{H}),-0.02(\mathrm{~s}, 6 \mathrm{H}) \mathrm{ppm}$.

${ }^{13} \mathbf{C}\left\{{ }^{1} \mathbf{H}\right\}$ NMR $\left(\mathrm{CDCl}_{3}, 151 \mathrm{MHz}, \mathrm{rt}\right): \delta=146.1,146.0,141.1,138.8,134.1,111.9,59.5,57.3,41.8,34.0$, $29.7,29.4,26.4,26.3,18.9,18.6,-3.1,-3.5,-3.8,-4.3 \mathrm{ppm}$.

IR (ATR): $\tilde{\boldsymbol{v}}=2953,2929,2857,1464,1337,1251,1218,1108,1002,828,777 \mathrm{~cm}^{-1}$.

HRMS $\left(\mathrm{MALDI}^{+}\right) \mathrm{m} / \mathrm{z}:[\mathrm{M}]^{+}$: calcd. for $\left[\mathrm{C}_{47} \mathrm{H}_{82} \mathrm{O}_{4} \mathrm{Si}_{4}\right]^{++}$: 822.5285; found 822.5295; correct isotope distribution.

\section{Crystal data}

Single crystalline specimen were obtained by slow diffusion of methanol into a chloroform solution of S6:

Colorless crystal (plate), dimensions $0.098 \times 0.067 \times 0.012 \mathrm{~mm}^{3}$, crystal system monoclinic, space group $\mathrm{P} 2{ }_{1} / \mathrm{n}, Z=4, a=10.3973(11) \AA, b=34.436(5) \AA, c=14.5834(16) \AA, \alpha=90^{\circ}, \beta=95.946(9)^{\circ}, \gamma=90^{\circ}$, $V=5193.3(10) \AA^{3}, \rho=1.053 \mathrm{~g} / \mathrm{cm}^{3}, T=200(2) \mathrm{K}, \Theta_{\max }=42.548^{\circ}, 15540$ reflections measured, 3583 unique $\left(\mathrm{R}_{\text {int }}=0.2178\right), 1485$ observed $(\mathrm{I}>2 \sigma(\mathrm{I})), \mu=1.34 \mathrm{~mm}^{-1}, T_{\min }=0.60, T_{\max }=1.46,520$ parameters refined, hydrogen atoms were treated using appropriate riding models, goodness of fit 0.91 for observed reflections, final residual values $\mathrm{R} 1(\mathrm{~F})=0.068, \mathrm{wR}\left(\mathrm{F}^{2}\right)=0.129$ for observed reflections, residual electron density -0.26 to $0.44 \mathrm{e}^{-3}$.

2,2,11,11-Tetramethyl-1,2,6,7,11,12-hexahydrobenzo[cd] indeno[7,1-hi]azulene-4,5,8,9-tetrol (S7) 

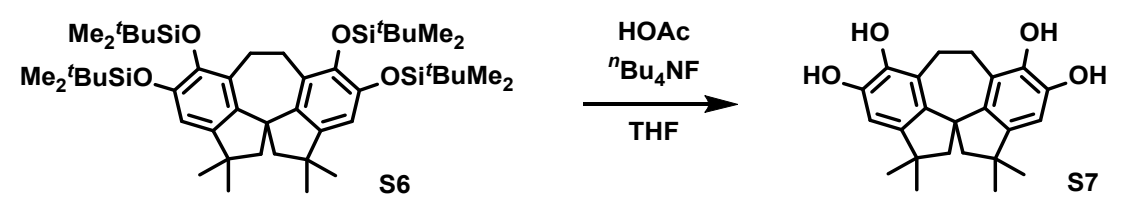

In a Schlenk tube under an atmosphere of argon was dissolved S6 (1.50 g, $1.82 \mathrm{mmol}, 1.00$ equiv.) in $15.0 \mathrm{~mL}$ anhydrous tetrahydrofuran. Acetic acid (438 $\mu \mathrm{L}, 459 \mathrm{mg}, 7.65 \mathrm{mmol}, 4.20$ equiv.) and thereafter tetra- $n$ butylammonium fluoride $\left(1.0 \mathrm{~mol} \mathrm{~L}^{-1}\right.$ in tetrahydrofuran, $7.65 \mathrm{~mL}, 2.00 \mathrm{~g}, 7.65 \mathrm{mmol}, 4.20$ equiv.) were added dropwise at $0{ }^{\circ} \mathrm{C}$ and the reaction mixture was stirred at $0{ }^{\circ} \mathrm{C}$ for $2 \mathrm{~h}$. The solvent was removed under reduced pressure, the crude product was dissolved in $30 \mathrm{~mL}$ ethyl acetate and washed with water $(5 \times 20 \mathrm{~mL})$. The organic phases were dried over magnesium sulfate and filtrated. The solvent was removed under reduced pressure and the crude product was absorbed on Celite. After flash column chromatography (dichloromethane/methanol 100:1 v/v -> 50:1) the product $\mathbf{S 7}$ was isolated as a colorless solid (538 $\mathrm{mg}$, $1.47 \mathrm{mmol}, 81 \%)$.

$\mathbf{R}_{f}=0.43\left(\mathrm{SiO}_{2} ;\right.$ dichloromethane/methanol 10:1 v/v).

Mp: $133^{\circ} \mathrm{C}$.

${ }^{1}$ H NMR (DMSO-d6, $\left.300 \mathrm{MHz}, \mathrm{rt}\right): \delta=8.82(\mathrm{~s}, 2 \mathrm{H}), 7.73(\mathrm{~s}, 2 \mathrm{H}), 3.15-3.24(\mathrm{~m}, 2 \mathrm{H}), 2.33(\mathrm{~d}, J=11.6 \mathrm{~Hz}$, 2H), $1.98-2.07(\mathrm{~m}, 2 \mathrm{H}), 1.67(\mathrm{~d}, J=11.6 \mathrm{~Hz}, 2 \mathrm{H}), 1.46(\mathrm{~s}, 6 \mathrm{H}), 1.05(\mathrm{~s}, 6 \mathrm{H}) \mathrm{ppm}$.

${ }^{13} \mathbf{C}\left\{{ }^{1} \mathbf{H}\right\}$ NMR $\left(\mathrm{CDCl}_{3}, 151 \mathrm{MHz}, \mathrm{rt}\right): \delta=145.0,144.4,141.5,137.7,129.1,107.0,60.2,58.2,42.3,34.4$, 29.9, 28.4 ppm.

IR (ATR): $\tilde{\boldsymbol{v}}=3252,2964,2917,2858,1593,1455,1325,1302,1231,1041,946,853 \mathrm{~cm}^{-1}$.

HRMS $\left(\mathrm{MALDI}^{+}\right) \mathrm{m} / z:[\mathrm{M}-\mathrm{H}]^{+}$: calcd. for $\left[\mathrm{C}_{23} \mathrm{H}_{25} \mathrm{O}_{4}\right]^{+}: 365.1747$; found 365.1753 ; correct isotope distribution.

2,2,11,11-Tetramethyl-1,2,6,7,11,12-hexahydrobenzo[cd]indeno[7,1-hi]azulene-4,5,8,9-tetrone (6)
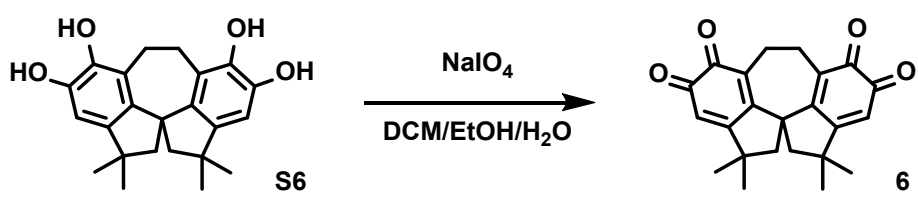
GP1 was applied to racemic biscatechol S7 $(25.0 \mathrm{mg}, 68.2 \mu \mathrm{mol}, 1.00$ equiv.) and sodium periodate (32.1 mg, $150 \mu \mathrm{mol}, 2.20$ equiv.) in $500 \mu \mathrm{L}$ ethanol, $1 \mathrm{~mL}$ dichloromethane and $1 \mathrm{~mL}$ distilled water. After workup, racemic bis-ortho-quinone 6 was isolated as a red solid (23.7 mg, $65.4 \mu \mathrm{mol}, 96 \%)$.

$\mathbf{R}_{\boldsymbol{f}}=0.19\left(\mathrm{SiO}_{2} ;\right.$ petroleum ether/ethyl acetate $\left.2: 1, \mathrm{v} / \mathrm{v}\right)$.

Mp: $\geq 247^{\circ} \mathrm{C}$ (decomposition).

${ }^{1} \mathbf{H}$ NMR $\left(\mathrm{CDCl}_{3}, 400 \mathrm{MHz}, \mathrm{rt}\right): \delta=6.20(\mathrm{~s}, 2 \mathrm{H}), 3.18-3.29(\mathrm{~m}, 2 \mathrm{H}), 2.54(\mathrm{~d}, J=12.9 \mathrm{~Hz}, 2 \mathrm{H}), 2.05-2.16$ (m, 2H), 1.89 (d, J=12.9 Hz, 2H), 1.58 (s, 6H), 1.31 (s, 6H) ppm.

${ }^{13} \mathbf{C}\left\{{ }^{1} \mathbf{H}\right\}$ NMR $\left(\mathrm{CDCl}_{3}, 101 \mathrm{MHz}, \mathrm{rt}\right): \delta=179.0,179.0,170.1,155.6,138.1,120.3,58.4,50.8,41.6,33.2$, 29.8, $23.2 \mathrm{ppm}$.

IR (ATR): $\tilde{\boldsymbol{v}}=2978,2925,2863,1660,1594,1337,1267,1243,876,749 \mathrm{~cm}^{-1}$.

HRMS $\left(\mathrm{ESI}^{+}\right) \mathrm{m} / z$ : $[\mathrm{M}]^{++}$: calcd. for $\left[\mathrm{C}_{23} \mathrm{H}_{22} \mathrm{NaO}_{4}\right]^{++}$: 385.1410; found 385.1419; correct isotope distribution.

\section{Crystal data}

Single crystalline specimen were obtained by slow diffusion of methanol into a chloroform solution of $\mathbf{6}$ :

Red crystal (brick), dimensions $0.152 \times 0.091 \times 0.060 \mathrm{~mm}^{3}$, crystal system monoclinic, space group C2/c, $Z=4, \quad a=19.4498(10) \AA, \quad b=9.0182(3) \AA, \quad c=13.1804(13) \AA, \quad \alpha=90^{\circ}, \quad \beta=127.754(3)^{\circ}, \quad \gamma=90^{\circ}$, $V=1827.9(2) \AA^{3}, \rho=1.317 \mathrm{~g} / \mathrm{cm}^{3}, T=200(2) \mathrm{K}, \Theta_{\max }=71.460^{\circ}, 5759$ reflections measured, 1741 unique $\left(R_{\text {int }}=0.0126\right), 1581$ observed $(\mathrm{I}>2 \sigma(\mathrm{I})), \mu=0.72 \mathrm{~mm}^{-1}, T_{\min }=0.67, T_{\max }=1.54,125$ parameters refined, hydrogen atoms were treated using appropriate riding models, goodness of fit 1.08 for observed reflections, final residual values $\mathrm{R} 1(\mathrm{~F})=0.040, \mathrm{wR}\left(\mathrm{F}^{2}\right)=0.104$ for observed reflections, residual electron density -0.22 to $0.22 \mathrm{e}^{-3}$.

\subsubsection{Homodimer Synthesis}

3,3,3',3',15,15'-Hexamethyl-6,6',13,13'-tetrakis \{[triisopropylsilyl]ethynyl\}-2,2',3,3'-tetrahydro-1,1'-spirobi[cyclopenta[b]naphtho[2,3-i]phenazine] (1a) 

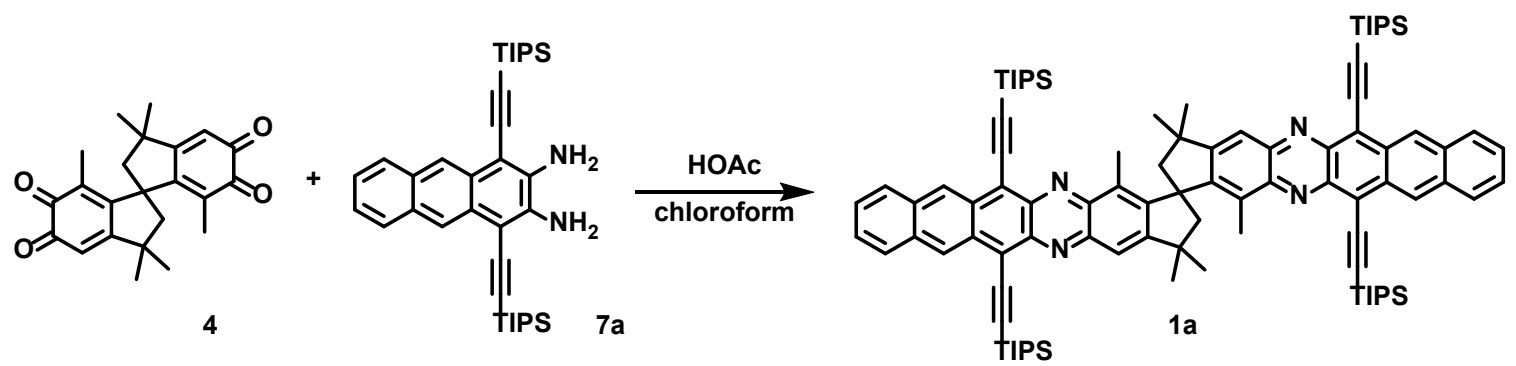

GP2 was applied to racemic bis-ortho-quinone 4 (40 mg, $110 \mu \mathrm{mol}, 1.00$ equiv.) and diamine 7a (125 mg, $220 \mu \mathrm{mol}, 2.00$ equiv.) in $1.50 \mathrm{~mL}$ chloroform and $1.50 \mathrm{~mL}$ acetic acid. Flash column chromatography $\left(\mathrm{SiO}_{2}\right.$; petroleum ether/diethyl ether 500:1 v/v -> 250:1) and gel permeation chromatography (toluene) yielded green solid 1a $(86.0 \mathrm{mg}, 60.1 \mu \mathrm{mol}, 55 \%)$.

$\mathbf{R}_{\boldsymbol{f}}=0.61\left(\mathrm{SiO}_{2} ;\right.$ petroleum ether/dichloromethane $\left.2: 1, \mathrm{v} / \mathrm{v}\right)$.

Mp: $\geq 350{ }^{\circ} \mathrm{C}$.

${ }^{1} \mathbf{H}$ NMR $\left(\mathrm{CDCl}_{3}, 300 \mathrm{MHz}, \mathrm{rt}\right): \delta=9.42(\mathrm{~s}, 4 \mathrm{H}), 7.93$ - $8.05(\mathrm{~m}, 4 \mathrm{H}), 7.90(\mathrm{~s}, 2 \mathrm{H}), 7.41-7.50(\mathrm{~m}, 4 \mathrm{H}), 2.77$ $(\mathrm{d}, J=13.1 \mathrm{~Hz}, 2 \mathrm{H}), 2.68(\mathrm{~d}, J=13.1 \mathrm{~Hz}, 2 \mathrm{H}), 2.36(\mathrm{~s}, 6 \mathrm{H}), 1.64(\mathrm{~s}, 6 \mathrm{H}), 1.61(\mathrm{~s}, 6 \mathrm{H}), 1.38-1.45(\mathrm{~m}, 42 \mathrm{H})$, $1.18-1.26(\mathrm{~m}, 42 \mathrm{H}) \mathrm{ppm}$.

${ }^{13} \mathbf{C}\left\{{ }^{1} \mathbf{H}\right\}$ NMR $\left(\mathrm{CDCl}_{3}, 151 \mathrm{MHz}, \mathrm{rt}\right): \delta=159.4,152.1,145.5,145.0,140.6,139.8,132.9,132.8,132.7$, $132.6,132.5,128.8,128.8,127.0,126.8,126.7,126.7,120.8,120.5,120.0,108.9,108.9,104.2,103.9,57.8$, $56.7,43.8,33.2,29.6,19.2,19.1,19.1,13.4,11.9,11.7 \mathrm{ppm}$.

IR (ATR): $\tilde{\boldsymbol{v}}=2940,2862,1459,1374,1012,879,751,740,673,659,584,460 \mathrm{~cm}^{-1}$.

HRMS $\left(\mathrm{MALDI}^{+}\right.$) $m / z:[\mathrm{M}+\mathrm{H}]^{+}$: calcd. for $\left[\mathrm{C}_{95} \mathrm{H}_{121} \mathrm{~N}_{4} \mathrm{Si}_{4}\right]^{+}:$1429.8663; found 1429.8675; correct isotope distribution.

\section{Crystal data}

Single crystalline specimen were obtained by slow diffusion of methanol into a chloroform solution of 1a:

Green crystal (plate), dimensions $0.170 \times 0.095 \times 0.022 \mathrm{~mm}^{3}$, crystal system monoclinic, space group Cc, $Z=24, \quad a=50.7427(18) \AA, \quad b=31.5332(8) \AA, c=41.0801(15) \AA, \quad \alpha=90^{\circ}, \beta=118.233(3)^{\circ}, \gamma=90^{\circ}$, $V=57911(4) \AA^{3}, \rho=1.053 \mathrm{~g} / \mathrm{cm}^{3}, T=200(2) \mathrm{K}, \Theta_{\max }=50.434^{\circ}, 124802$ reflections measured, 49327 unique $\left(R_{\text {int }}=0.1907\right), 27191$ observed $(\mathrm{I}>2 \sigma(\mathrm{I})), \mu=1.53 \mathrm{~mm}^{-1}, T_{\min }=0.64, T_{\max }=1.50,5756$ parameters 
refined, hydrogen atoms were treated using appropriate riding models, goodness of fit 1.10 for observed reflections, final residual values $\mathrm{R} 1(\mathrm{~F})=0.108, \mathrm{wR}\left(\mathrm{F}^{2}\right)=0.213$ for observed reflections, residual electron density -0.30 to $0.55 \mathrm{e}^{-3}$.

3,3,3',3',15,15'-Hexamethyl-6,6',13,13'-tetrakis \{[triisopropylsilyl]ethynyl $\}-2,2$ ',3,3'-tetrahydro-1,1'-spirobi[cyclopenta $[b]$ quinoxalino[2,3-i]phenazine] (1b)

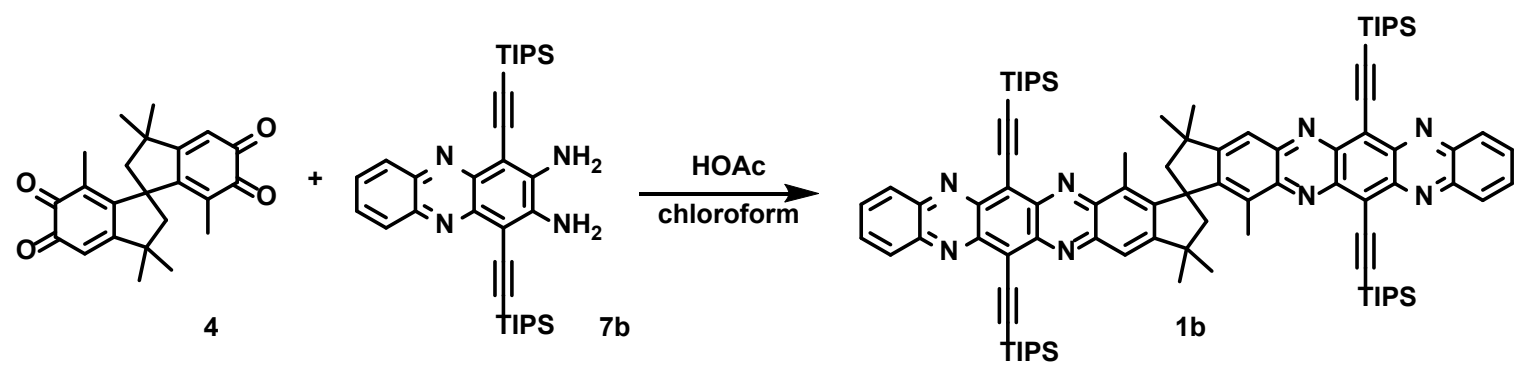

GP2 was applied to racemic bis-ortho-quinone 4 (40.0 mg, $110 \mu \mathrm{mol}, 1.00$ equiv.) and diamine $7 \mathbf{b}$ (125 mg, $220 \mu \mathrm{mol}, 2.00$ equiv.) in $1.50 \mathrm{~mL}$ chloroform and $1.50 \mathrm{~mL}$ acetic acid. Flash column chromatography $\left(\mathrm{SiO}_{2}\right.$; petroleum ether/diethyl ether 250:1 v/v -> 100:1 -> 50:1) and gel permeation chromatography (toluene) yielded green solid $\mathbf{1 b}$ (69.3 mg, $48.3 \mu \mathrm{mol}, 44 \%)$.

$\mathbf{R}_{\boldsymbol{f}}=0.13\left(\mathrm{SiO}_{2}\right.$; petroleum ether/dichloromethane $\left.2: 1, \mathrm{v} / \mathrm{v}\right)$.

Mp: $\geq 350^{\circ} \mathrm{C}$.

${ }^{1} \mathbf{H}$ NMR $\left(\mathrm{CDCl}_{3}, 600 \mathrm{MHz}, \mathrm{rt}\right): \delta=8.20$ - $8.24(\mathrm{~m}, 2 \mathrm{H}), 8.15-8.19(\mathrm{~m}, 2 \mathrm{H}), 7.96(\mathrm{~s}, 2 \mathrm{H}), 7.78-7.84(\mathrm{~m}$, 4H), $2.78(\mathrm{~d}, J=13.2 \mathrm{~Hz}, 2 \mathrm{H}), 2.70(\mathrm{~d}, J=13.2 \mathrm{~Hz}, 2 \mathrm{H}), 2.39(\mathrm{~s}, 6 \mathrm{H}), 1.76(\mathrm{~s}, 6 \mathrm{H}), 1.63(\mathrm{~s}, 6 \mathrm{H}), 1.39-1.44$ (m, 42H), 1.19 - $1.24(\mathrm{~m}, 42 \mathrm{H}) \mathrm{ppm}$.

${ }^{13} \mathbf{C}\left\{{ }^{1} \mathbf{H}\right\}$ NMR $\left(\mathrm{CDCl}_{3}, 151 \mathrm{MHz}, \mathrm{rt}\right): \delta=160.4,152.9,146.0,145.5,145.4,145.4,143.1,143.0,142.5$, $141.7,132.7,131.9,131.9,130.6,130.6,123.0,122.1,120.7,111.9,111.7,103.3,103.1,57.9,56.7,44.0$, $33.2,29.5,19.2,19.0,13.4,12.0,11.7 \mathrm{ppm}$.

IR (ATR): $\tilde{\boldsymbol{v}}=2940,2862,1456,1383,1084,1030,1012,880,753,738,673,588 \mathrm{~cm}^{-1}$.

HRMS $\left(\mathrm{MALDI}^{+}\right) m / z:[\mathrm{M}+2 \mathrm{H}]^{++}$: calcd. for $\left[\mathrm{C}_{91} \mathrm{H}_{118} \mathrm{~N}_{8} \mathrm{Si}_{4}\right]^{++}: 1434.8551$; found 1434.8567; correct isotope distribution. 
6,6',9,9,9',9'-Hexamethyl-4,4',12,12'-tetrakis \{[triisopropylsilyl]ethynyl $\}-8,8$ ',9,9'-tetrahydro-7,7'-spirobi[cyclopenta $[b][1,2,5]$ thiadiazolo[3,4-i]phenazine $](\mathbf{1 c})$
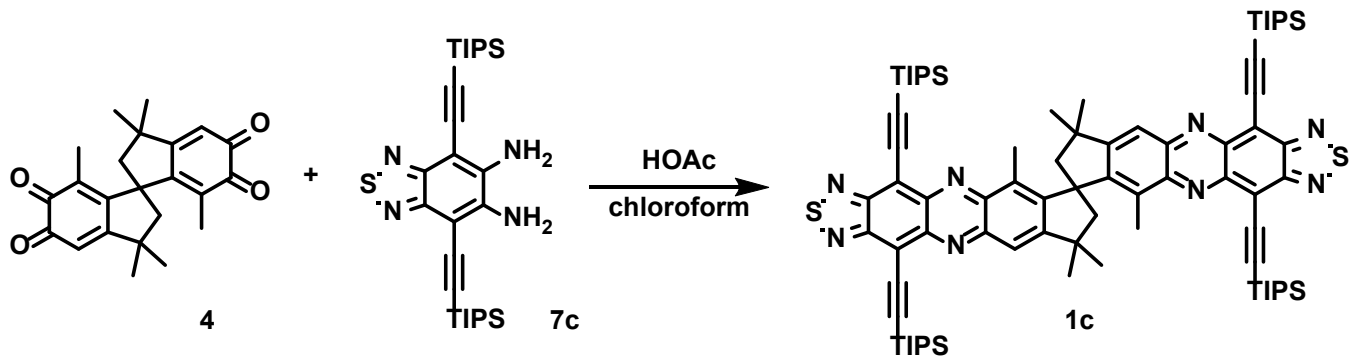

GP2 was applied to racemic bis-ortho-quinone 4 (50.0 mg, $137 \mu \mathrm{mol}, 1.00$ equiv.) and diamine 7c (145 mg, $274 \mu \mathrm{mol}, 2.00$ equiv.) in $1.50 \mathrm{~mL}$ chloroform and $1.50 \mathrm{~mL}$ acetic acid. Flash column chromatography $\left(\mathrm{SiO}_{2}\right.$; petroleum ether/diethyl ether 250:1 v/v -> 100:1 -> 50:1) and gel permeation chromatography (toluene) yielded green solid 1c (87.3 mg, $137 \mu \mathrm{mol}, 47 \%)$.

$\mathbf{R}_{\boldsymbol{f}}=0.39\left(\mathrm{SiO}_{2} ;\right.$ petroleum ether/dichloromethane $\left.2: 1, \mathrm{v} / \mathrm{v}\right)$.

Mp: $334^{\circ} \mathrm{C}$.

${ }^{1} \mathbf{H}$ NMR $\left(\mathrm{CDCl}_{3}, 600 \mathrm{MHz}, \mathrm{rt}\right): \delta=7.86(\mathrm{~s}, 2 \mathrm{H}), 2.74(\mathrm{~d}, J=13.6 \mathrm{~Hz}, 2 \mathrm{H}), 2.68(\mathrm{~d}, J=13.6 \mathrm{~Hz}, 2 \mathrm{H}), 2.32$ (s, 6H), $1.73(\mathrm{~s}, 6 \mathrm{H}), 1.61(\mathrm{~s}, 6 \mathrm{H}), 1.32$ - $1.42(\mathrm{~m}, 42 \mathrm{H}), 1.13$ - $1.22(\mathrm{~m}, 42 \mathrm{H}) \mathrm{ppm}$.

${ }^{13} \mathbf{C}\left\{{ }^{1} \mathbf{H}\right\} \mathbf{N M R}\left(\mathrm{CDCl}_{3}, 151 \mathrm{MHz}, \mathrm{rt}\right): \delta=160.5,155.0,154.7,153.0,146.0,145.5,142.3,141.4,132.5$, $120.5,114.6,113.6,111.0,111.0,102.3,102.0,57.8,56.6,43.9,33.1,29.5,19.1,18.9,13.3,11.8,11.5$ ppm.

IR (ATR): $\tilde{\boldsymbol{v}}=2957,2942,2865,2358,1461,1365,1027,882,741,669 \mathrm{~cm}^{-1}$.

HRMS $\left(\mathrm{MALDI}^{+}\right) \mathrm{m} / z$ : $[\mathrm{M}+2 \mathrm{H}]^{+}$: calcd. for $\left[\mathrm{C}_{79} \mathrm{H}_{110} \mathrm{~N}_{8} \mathrm{~S}_{2} \mathrm{Si}_{4}\right]^{+}$: 1346.7366; found 1346.7369; correct isotope distribution. 
9,9,12,12-Tetramethyl-6,15,22,28-tetrakis $\{[$ triisopropylsilyl] ethynyl $\}-9,10,11,12$-tetrahydro-24H,26H-25oxa-7,14,23,27-tetraazatetraceno[2"',3"':5",6"]indeno[1",7":1',8',7']cycloocta[1',-2',3':3,4]indeno[5,6-b]tetracene $(\mathbf{2 a})$

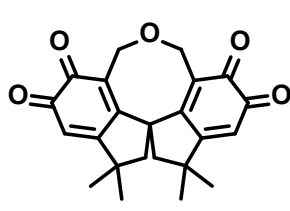

5

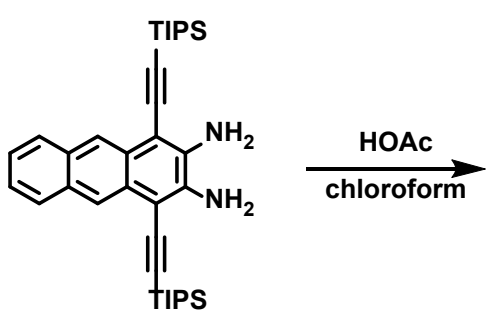

$7 a$

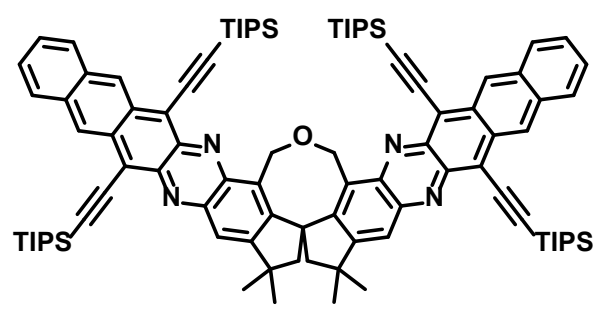

2a

GP2 was applied to racemic bis-ortho-quinone $5(50.0 \mathrm{mg}, 132 \mu \mathrm{mol}, 1.00$ equiv.) and diamine $7 \mathbf{a}(150 \mathrm{mg}$, $264 \mu \mathrm{mol}, 2.00$ equiv.) in $1.50 \mathrm{~mL}$ chloroform and $1.50 \mathrm{~mL}$ acetic acid. Flash column chromatography $\left(\mathrm{SiO}_{2}\right.$; petroleum ether/diethyl ether 250:1 v/v) and gel permeation chromatography (toluene) yielded green solid 2a (105 mg, $73.0 \mu \mathrm{mol}, 55 \%)$.

$\mathbf{R}_{\boldsymbol{f}}=0.65\left(\mathrm{SiO}_{2}\right.$; petroleum ether/dichloromethane $\left.2: 1, \mathrm{v} / \mathrm{v}\right)$.

Mp: $\geq 350{ }^{\circ} \mathrm{C}$.

${ }^{1} \mathbf{H}$ NMR $\left(\mathrm{CDCl}_{3}, 300 \mathrm{MHz}, \mathrm{rt}\right): \delta=9.47$ (s, 2H), 9.43 (s, 2H), 7.97 - 8.07 (m, 4H), 7.96 (s, 2H), 7.44 - 7.51 $(\mathrm{m}, 4 \mathrm{H}), 6.23(\mathrm{~d}, J=11.9 \mathrm{~Hz}, 2 \mathrm{H}), 4.58(\mathrm{~d}, J=11.9 \mathrm{~Hz}, 2 \mathrm{H}), 2.80(\mathrm{~d}, J=13.0 \mathrm{~Hz}, 2 \mathrm{H}), 2.34(\mathrm{~d}, J=13.0 \mathrm{~Hz}$, 2H), $1.80(\mathrm{~s}, 6 \mathrm{H}), 1.60(\mathrm{~s}, 6 \mathrm{H}), 1.39$ - $1.43(\mathrm{~m}, 42 \mathrm{H}), 1.22$ - $1.27(\mathrm{~m}, 42 \mathrm{H}) \mathrm{ppm}$.

${ }^{13} \mathbf{C}\left\{{ }^{1} \mathbf{H}\right\}$ NMR $\left(\mathrm{CDCl}_{3}, 101 \mathrm{MHz}, \mathrm{rt}\right): \delta=157.5,156.3,145.6,144.3,140.7,140.2,133.0(2 \mathrm{C}), 132.9,132.6$, $128.9,128.8,127.1,126.8,126.8,126.7,125.7,123.0,121.1,120.3,109.3,109.0,104.2,104.0,59.3,57.1$, $56.7,42.8,32.6,31.3,19.2,19.1,11.9,11.7 \mathrm{ppm}$.

IR (ATR): $\tilde{\boldsymbol{v}}=2940,2863,1460,1375,1013,880,751,670,661,585,460 \mathrm{~cm}^{-1}$.

HRMS $\left(\mathrm{MALDI}^{+}\right.$) $m / z:[\mathrm{M}+\mathrm{H}]^{+}$: calcd. for $\left[\mathrm{C}_{95} \mathrm{H}_{119} \mathrm{~N}_{4} \mathrm{OSi}\right]^{+}:$1443.8455; found 1443.8406; correct isotope distribution. 
9,9,12,12-Tetramethyl-6,15,22,28-tetrakis \{[triisopropylsilyl] ethynyl $\}-9,10,11,12$-tetrahydro-24H,26H-25oxa-5,7,14,16,21,23,27,29-octaazatetraceno[2"',3"':5",6"]indeno[1",7"::1',8',7']-cycloocta[1',2',3':3,4]indeno$[5,6-b]$ tetracene $(\mathbf{2 b})$

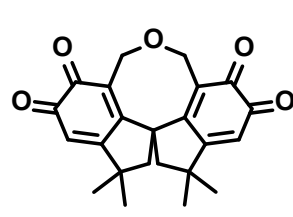

5

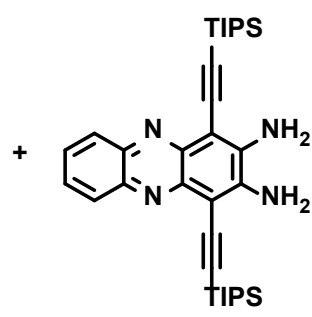

$7 \mathbf{b}$

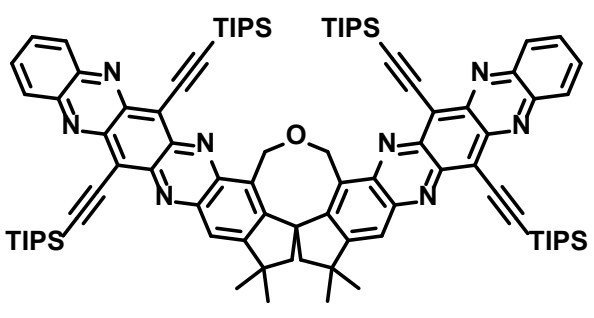

2b

GP2 was applied to racemic bis-ortho-quinone 5 (50.0 mg, $132 \mu \mathrm{mol}, 1.00$ equiv.) and diamine $7 \mathbf{b}$ (151 mg, $264 \mu \mathrm{mol}, 2.00$ equiv.) in $1.50 \mathrm{~mL}$ chloroform and $1.50 \mathrm{~mL}$ acetic acid. Flash column chromatography $\left(\mathrm{SiO}_{2}\right.$; petroleum ether/diethyl ether 250:1 v/v $->100: 1->50: 1$ ) and gel permeation chromatography (toluene) yielded green solid $\mathbf{2 b}(101 \mathrm{mg}, 69.7 \mu \mathrm{mol}, 53 \%)$.

$\mathbf{R}_{\boldsymbol{f}}=0.20\left(\mathrm{SiO}_{2}\right.$; petroleum ether/dichloromethane 2:1, v/v).

Mp: $\geq 350{ }^{\circ} \mathrm{C}$.

${ }^{1} \mathbf{H}$ NMR $\left(\mathrm{CDCl}_{3}, 400 \mathrm{MHz}, \mathrm{rt}\right): \delta=8.17$ - $8.26(\mathrm{~m}, 4 \mathrm{H}), 8.02(\mathrm{~s}, 2 \mathrm{H}), 7.78-7.86(\mathrm{~m}, 4 \mathrm{H}), 6.27(\mathrm{~d}, J=$ $12.1 \mathrm{~Hz}, 2 \mathrm{H}), 4.60(\mathrm{~d}, J=12.1 \mathrm{~Hz}, 2 \mathrm{H}), 2.82(\mathrm{~d}, J=13.1 \mathrm{~Hz}, 2 \mathrm{H}), 2.37(\mathrm{~d}, J=13.1 \mathrm{~Hz}, 2 \mathrm{H}), 1.82(\mathrm{~s}, 6 \mathrm{H})$, $1.62(\mathrm{~s}, 6 \mathrm{H}), 1.38-1.43(\mathrm{~m}, 42 \mathrm{H}), 1.23-1.27(\mathrm{~m}, 42 \mathrm{H}) \mathrm{ppm}$.

${ }^{13} \mathbf{C}\left\{{ }^{1} \mathbf{H}\right\}$ NMR $\left(\mathrm{CDCl}_{3}, 101 \mathrm{MHz}, \mathrm{rt}\right): \delta=158.5,157.0,146.1,145.5,145.5,144.8,143.3,143.0,142.6$, $142.1,132.0,131.9,130.7,130.7,125.8,123.2$, 123.2, 122.4, 112.5, 111.9, 103.4, 103.2, 59.5, 57.0, 56.7, $43.0,32.6,31.3,19.2,19.1,12.0,11.8 \mathrm{ppm}$.

IR (ATR): $\tilde{\boldsymbol{v}}=2940,2862,1455,1415,1383,1363,1224,1029,998,879,752,663,589 \mathrm{~cm}^{-1}$.

HRMS $\left(\mathrm{ESI}^{+}\right) \mathrm{m} / z$ : $[\mathrm{M}+\mathrm{H}]^{+}$: calcd. for $\left[\mathrm{C}_{91} \mathrm{H}_{115} \mathrm{~N}_{8} \mathrm{OSi}\right]^{+}:$1447.8265; found 1447.8283; correct isotope distribution. 
7,7,10,10-tetramethyl-4,13,17,23-tetrakis $\{[$ triisopropylsilyl]ethynyl $\}-7,8,9,10$-tetrahydro-19H,-21H-20oxa-2,15-dithia-1,3,5,12,14,16,18,22-octaazacyclopenta[b]cyclopenta-[6"',7"']naphtho[2"',3"':5",6"]indeno[1", 7": 1', 8',7']cycloocta[1',2',3':3,4]indeno[5,6-g]-naphthalene (2c)

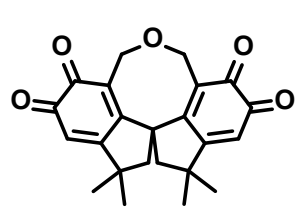

5

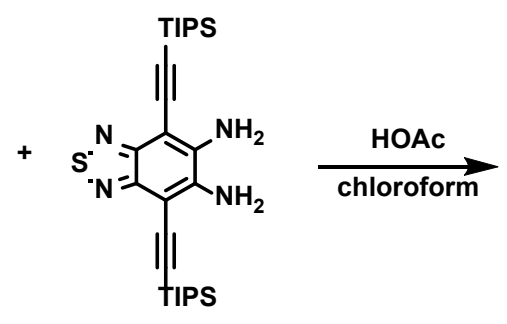

7c

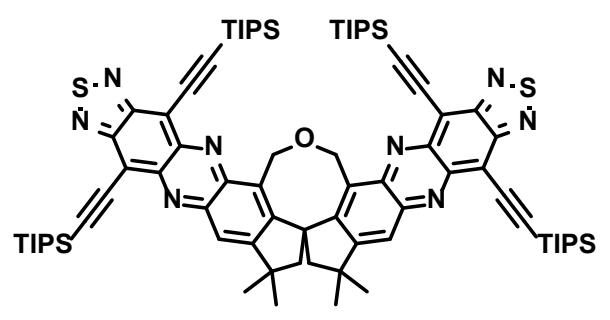

2c

GP2 was applied to racemic bis-ortho-quinone 5 (50.0 mg, $132 \mu \mathrm{mol}, 1.00$ equiv.) and diamine 7c (139 mg, $264 \mu \mathrm{mol}, 2.00$ equiv.) in $1.50 \mathrm{~mL}$ chloroform and $1.50 \mathrm{~mL}$ acetic acid. Flash column chromatography $\left(\mathrm{SiO}_{2}\right.$; petroleum ether/diethyl ether 250:1 v/v -> 100:1 -> 50:1) and gel permeation chromatography (toluene) yielded green solid $2 \mathrm{c}(112 \mathrm{mg}, 82.0 \mu \mathrm{mol}, 62 \%)$.

$\mathbf{R}_{\boldsymbol{f}}=0.32\left(\mathrm{SiO}_{2}\right.$; petroleum ether/dichloromethane $\left.2: 1, \mathrm{v} / \mathrm{v}\right)$.

Mp: $\geq 350{ }^{\circ} \mathrm{C}$.

${ }^{1} \mathbf{H}$ NMR $\left(\mathrm{CDCl}_{3}, 400 \mathrm{MHz}, \mathrm{rt}\right): \delta=7.93(\mathrm{~s}, 2 \mathrm{H}), 6.17(\mathrm{~d}, J=12.3 \mathrm{~Hz}, 2 \mathrm{H}), 4.54(\mathrm{~d}, J=12.3 \mathrm{~Hz}, 2 \mathrm{H}), 2.80$ $(\mathrm{d}, J=13.1 \mathrm{~Hz}, 2 \mathrm{H}), 2.33(\mathrm{~d}, J=13.1 \mathrm{~Hz}, 2 \mathrm{H}), 1.80(\mathrm{~s}, 6 \mathrm{H}), 1.59(\mathrm{~s}, 6 \mathrm{H}), 1.33-1.37(\mathrm{~m}, 42 \mathrm{H}), 1.16-1.22$ (m, 42H) ppm.

${ }^{13} \mathbf{C}\left\{{ }^{1} \mathbf{H}\right\}$ NMR $\left(\mathrm{CDCl}_{3}, 101 \mathrm{MHz}, \mathrm{rt}\right): \delta=158.6,157.1,155.2,154.7,146.1,144.7,142.5,141.8,125.6$, 123.0, 114.8, 113.9, 111.6, 111.1, 102.4, 102.1, 59.4, 56.9, 56.6, 42.9, 32.6, 31.3, 19.1, 19.0, 11.8, 11.6 ppm. IR (ATR): $\tilde{\boldsymbol{v}}=2940,2863,1453,1414,1364,1231,1026,996,918,879,741,659,622,578,464 \mathrm{~cm}^{-1}$.

HRMS $\left(\mathrm{ESI}^{+}\right) \mathrm{m} / z:[\mathrm{M}+\mathrm{H}]^{+}$: calcd. for $\left[\mathrm{C}_{79} \mathrm{H}_{107} \mathrm{~N}_{8} \mathrm{OSS}_{2} \mathrm{Si}_{4}\right]^{+}: 1359.7081$; found 1359.7096; correct isotope distribution.

\section{Crystal data}

Single crystalline specimen were obtained by slow diffusion of methanol into a chloroform solution of 2c: 
Dark green crystal (brick), dimensions 0.080 × 0.070 × $0.020 \mathrm{~mm}^{3}$, crystal system triclinic, space group P $\overline{1}$, $Z=2, \quad a=13.9273(10) \AA, \quad b=15.3829(11) \AA, \quad c=20.0465(14) \AA, \quad \alpha=96.310(5)^{\circ}, \quad \beta=108.019(5)^{\circ}$, $\gamma=89.662(6)^{\circ}, \quad V=4057.6(5) \AA^{3}, \rho=1.113 \mathrm{~g} / \mathrm{cm}^{3}, T=200(2) \mathrm{K}, \Theta_{\max }=60.005^{\circ}, 43002$ reflections measured, 11578 unique $\left(R_{\text {int }}=0.0936\right), 6110$ observed $(\mathrm{I}>2 \sigma(\mathrm{I})), \mu=1.51 \mathrm{~mm}^{-1}, T_{\min }=0.63, T_{\max }=1.23$, 876 parameters refined, hydrogen atoms were treated using appropriate riding models, goodness of fit 0.87 for observed reflections, final residual values $\mathrm{R} 1(\mathrm{~F})=0.070, \mathrm{wR}\left(\mathrm{F}^{2}\right)=0.153$ for observed reflections, residual electron density -0.50 to $0.50 \mathrm{e}^{-3}$.

9,9,12,12-Tetramethyl-6,15,22,27-tetrakis \{[triisopropylsilyl]ethynyl\}-9,10,11,12,24,25-hexahydronaphtho[2,3-i]naphtho[2"',3"':6",7"]quinoxalino[2",3":5',6']indeno-[1',7':3a,4,5]azuleno[8,1-ab]phenazine (3a)

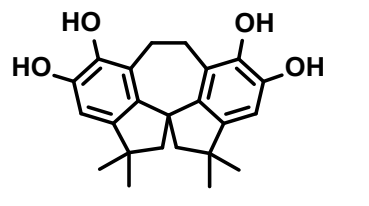

56
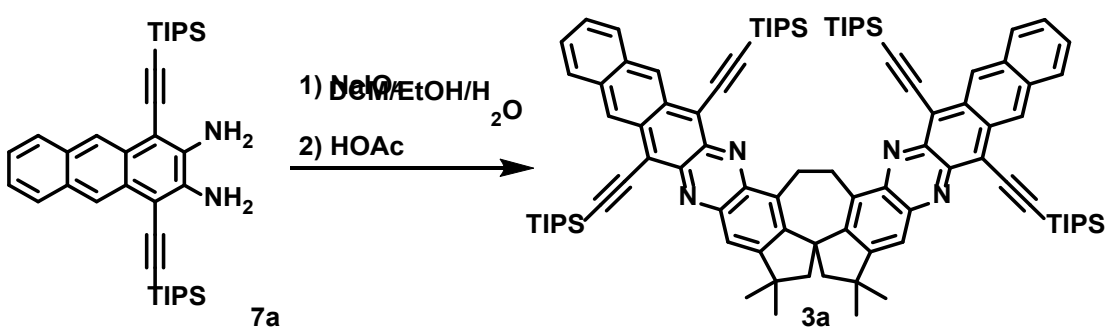

Following GP3, racemic bis-ortho-quinone was generated in situ from biscatechol S6 (50.0 mg, $136 \mu \mathrm{mol}$, 1.00 equiv.) and sodium periodate $(64.2 \mathrm{mg}, 300 \mu \mathrm{mol}, 2.20$ equiv.) in $500 \mu \mathrm{L}$ ethanol, $1.50 \mathrm{~mL}$ dichloromethane and $1.00 \mathrm{~mL}$ distilled water. After extraction, the organic layer and $4.00 \mathrm{~mL}$ acetic acid were reacted with ortho-diamine 7a (155 mg, $273 \mu \mathrm{mol}, 2.00$ equiv.) Flash column chromatography $\left(\mathrm{SiO}_{2}\right.$; petroleum ether/diethyl ether 250:1 v/v) and gel permeation chromatography (toluene) yielded green solid 3a (104 mg, $72.5 \mu \mathrm{mol}, 53 \%)$.

$\mathbf{R}_{\boldsymbol{f}}=0.67\left(\mathrm{SiO}_{2} ;\right.$ petroleum ether/dichloromethane $\left.2: 1, \mathrm{v} / \mathrm{v}\right)$.

Mp: $\geq 350{ }^{\circ} \mathrm{C}$.

${ }^{1} \mathbf{H}$ NMR $\left(\mathrm{CDCl}_{3}, 400 \mathrm{MHz}, \mathrm{rt}\right): \delta=9.49(\mathrm{~s}, 2 \mathrm{H}), 9.44(\mathrm{~s}, 2 \mathrm{H}), 8.00$ - 8.09 (m, 4H), 7.79 (s, 2H), $7.44-8.52$ $(\mathrm{m}, 4 \mathrm{H}), 4.84(\mathrm{~d}, J=14.8 \mathrm{~Hz}, 2 \mathrm{H}), 2.92(\mathrm{~d}, J=14.8 \mathrm{~Hz}, 2 \mathrm{H}), 2.81(\mathrm{~d}, J=12.8 \mathrm{~Hz}, 2 \mathrm{H}), 2.19(\mathrm{~d}, J=12.8 \mathrm{~Hz}$, 2H), 1.89 (s, 6H), 1.44 (s, 6H), 1.38 - $1.48(\mathrm{~m}, 42 \mathrm{H}), 1.28$ - $1.32(\mathrm{~m}, 42 \mathrm{H}) \mathrm{ppm}$. 
${ }^{13} \mathbf{C}\left\{{ }^{1} \mathbf{H}\right\}$ NMR $\left(\mathrm{CDCl}_{3}, 101 \mathrm{MHz}, \mathrm{rt}\right): \delta=159.6,148.4,145.8,144.5,140.6,139.9,139.7,132.9,132.8$, $132.7,132.5,128.9,128.8,127.0,126.8,126.7,126.6,120.8,120.3,120.1,108.9,108.6,104.3,104.1,60.6$, $54.9,42.4,34.2,30.5,27.7,19.2,19.1,11.9,11.8 \mathrm{ppm}$.

IR (ATR): $\tilde{\boldsymbol{v}}=2940,2862,1460,1374,1139,1011,994,879,750,740,675,660,582,459 \mathrm{~cm}^{-1}$.

HRMS (MALDI $\left.{ }^{+}\right) m / z:[\mathrm{M}]^{++}$: calcd. for $\left[\mathrm{C}_{95} \mathrm{H}_{118} \mathrm{~N}_{4} \mathrm{Si}_{4}\right]^{++}:$1426.8428; found 1426.8428; correct isotope distribution.

9,9,12,12-Tetramethyl-6,15,22,27-tetrakis $\{$ [triisopropylsilyl]ethynyl $\}-9,10,11,12,24,25$-hexahydroquinoxalino[2,3-i]quinoxalino[2"',3"':6",7"]quinoxalino[2",3":5',6']indeno-[1',7':3a,4,5]azule-no[8,1-ab]phenazine (3b)
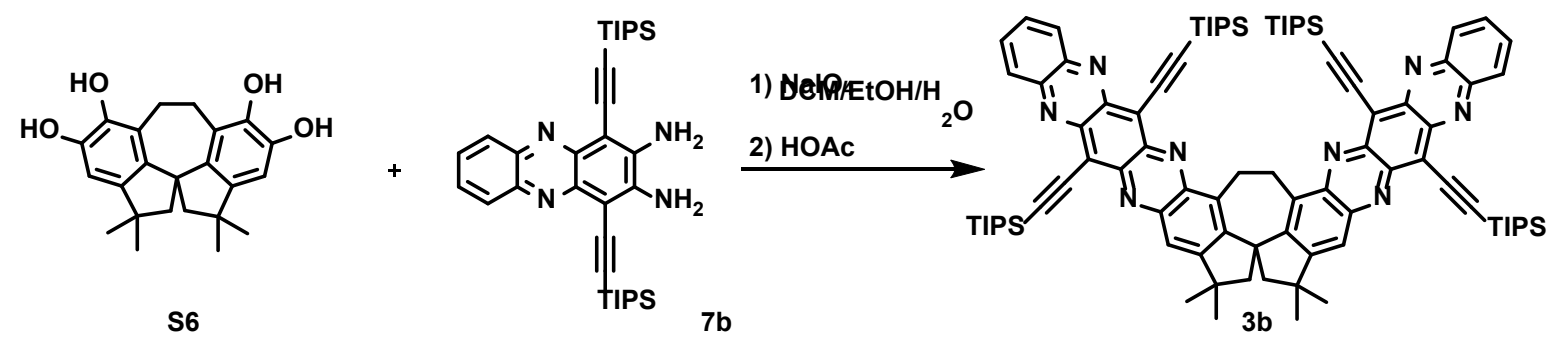

Following GP3, racemic bis-ortho-quinone was generated in situ from biscatechol S6 (50.0 mg, $136 \mu \mathrm{mol}$, 1.00 equiv.) and sodium periodate $(64.2 \mathrm{mg}, 300 \mu \mathrm{mol}, 2.20$ equiv.) in $500 \mu \mathrm{L}$ ethanol, $1.50 \mathrm{~mL}$ dichloromethane and $1.00 \mathrm{~mL}$ distilled water. After extraction, the organic layer and $4.00 \mathrm{~mL}$ acetic acid were reacted with ortho-diamine $7 \mathbf{b}\left(156 \mathrm{mg}, 273 \mu \mathrm{mol}, 2.00\right.$ equiv.) Flash column chromatography $\left(\mathrm{SiO}_{2}\right.$; petroleum ether/diethyl ether 250:1 v/v -> 100:1 -> 50:1) and gel permeation chromatography (toluene) yielded green solid $3 \mathbf{b}(97.0 \mathrm{mg}, 67.7 \mu \mathrm{mol}, 50 \%)$.

$\mathbf{R}_{\boldsymbol{f}}=0.11\left(\mathrm{SiO}_{2}\right.$; petroleum ether/dichloromethane 2:1, v/v).

Mp: $\geq 350{ }^{\circ} \mathrm{C}$. 
${ }^{1} \mathbf{H}$ NMR $\left(\mathrm{CDCl}_{3}, 400 \mathrm{MHz}, \mathrm{rt}\right): \delta=8.28-8.19(\mathrm{~m}, 4 \mathrm{H}), 7.86-8.80(\mathrm{~m}, 6 \mathrm{H}), 4.87(\mathrm{~d}, J=14.9 \mathrm{~Hz}, 2 \mathrm{H}), 2.96$ $(\mathrm{d}, J=14.9 \mathrm{~Hz}, 2 \mathrm{H}), 2.83(\mathrm{~d}, J=12.6 \mathrm{~Hz}, 2 \mathrm{H}), 2.21$ (d, $J=12.6 \mathrm{~Hz}, 2 \mathrm{H}), 1.91(\mathrm{~s}, 6 \mathrm{H}), 1.46(\mathrm{~s}, 6 \mathrm{H}), 1.38$ $1.43(\mathrm{~m}, 42 \mathrm{H}), 1.27-1.32(\mathrm{~m}, 42 \mathrm{H}) \mathrm{ppm}$.

${ }^{13} \mathbf{C}\left\{{ }^{1} \mathbf{H}\right\}$ NMR $\left(\mathrm{CDCl}_{3}, 101 \mathrm{MHz}, \mathrm{rt}\right): \delta=160.6,149.2,146.3,145.4,145.3,145.0,143.2,142.9,142.6$, $141.8,139.8,131.9,131.8,130.7,130.7,123.0,122.3,120.3,111.7,111.7,103.5,103.2,60.7,54.8,42.5$, $34.2,30.5,27.6,19.2,19.1,12.0,11.8 \mathrm{ppm}$.

IR (ATR): $\tilde{\boldsymbol{v}}=2957,2941,2864,1456,1384,1052,1028,881,755,676,591 \mathrm{~cm}^{-1}$.

HRMS $\left(\mathrm{MALDI}^{+}\right) \mathrm{m} / z$ : $[\mathrm{M}]^{++}$: calcd. for $\left[\mathrm{C}_{91} \mathrm{H}_{114} \mathrm{~N}_{8} \mathrm{Si}_{4}\right]^{++}: 1430.8238$; found 1430.8249; correct isotope distribution.

7,7,10,10-Tetramethyl-4,13,17,22-tetrakis $\{[$ triisopropylsilyl]ethynyl $\}-7,8,9,10,19,20$-hexahydro[1,2,5]thiadiazolo[3,4-i][1,2,5]thiadiazolo[3"',4"':6",7"]quinoxalino[2",3":5',6']indeno[1',-7':3a,4,5]azuleno[8,1-ab]phenazine (3c)
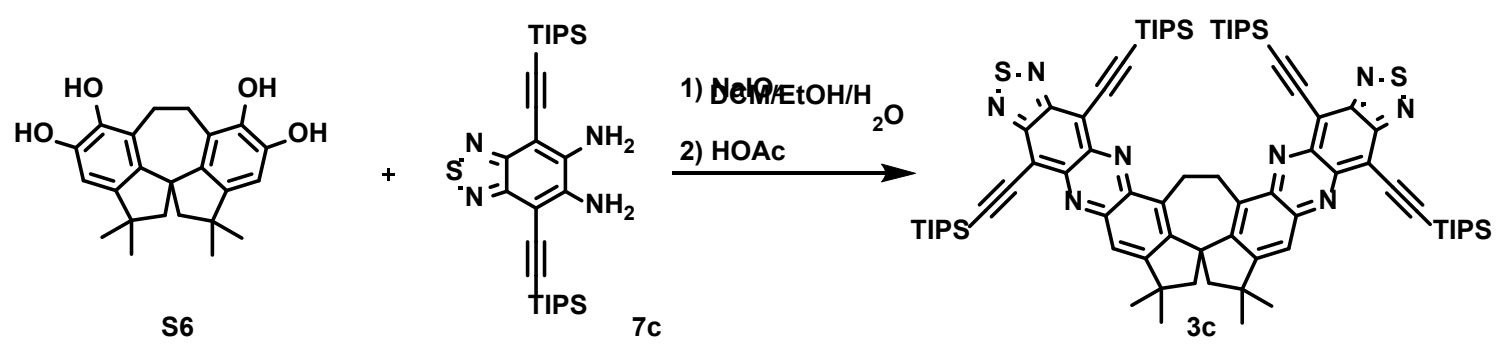

Following GP3, racemic bis-ortho-quinone was generated in situ from biscatechol S6 (40.0 mg, $109 \mu \mathrm{mol}$, 1.00 equiv.) and sodium periodate $(51.4 \mathrm{mg}, 240 \mu \mathrm{mol}, 2.20$ equiv.) in $500 \mu \mathrm{L}$ ethanol, $1.50 \mathrm{~mL}$ dichloromethane and $1.00 \mathrm{~mL}$ distilled water. After extraction, the organic layer and $4.00 \mathrm{~mL}$ acetic acid were reacted with ortho-diamine 7c (115 mg, $218 \mu \mathrm{mol}, 2.00$ equiv.) Flash column chromatography ( $\mathrm{SiO}_{2}$; petroleum ether/diethyl ether 250:1 v/v $->100: 1->50: 1)$ and gel permeation chromatography (toluene) yielded green solid 3c (70.3 mg, $52.3 \mu \mathrm{mol}, 48 \%)$.

$\mathbf{R}_{\boldsymbol{f}}=0.26\left(\mathrm{SiO}_{2} ;\right.$ petroleum ether/dichloromethane $\left.2: 1, \mathrm{v} / \mathrm{v}\right)$.

Mp: $\geq 350{ }^{\circ} \mathrm{C}$. 
${ }^{1} \mathbf{H}$ NMR $\left(\mathrm{CDCl}_{3}, 400 \mathrm{MHz}, \mathrm{rt}\right): \delta=7.75(\mathrm{~s}, 2 \mathrm{H}), 4.76(\mathrm{~d}, J=14.9 \mathrm{~Hz}, 2 \mathrm{H}), 2.87(\mathrm{~d}, J=14.9 \mathrm{~Hz}, 2 \mathrm{H}), 2.81$ $(\mathrm{d}, J=12.5 \mathrm{~Hz}, 2 \mathrm{H}), 2.17(\mathrm{~d}, J=12.5 \mathrm{~Hz}, 2 \mathrm{H}), 1.89$ (s, 6H), $1.43(\mathrm{~s}, 6 \mathrm{H}), 1.31-1.37$ (m, 42H), $1.20-1.26$ $(\mathrm{m}, 42 \mathrm{H}) \mathrm{ppm}$.

${ }^{13} \mathbf{C}\left\{{ }^{1} \mathbf{H}\right\}$ NMR $\left(\mathrm{CDCl}_{3}, 101 \mathrm{MHz}, \mathrm{rt}\right): \delta=160.7,155.1,154.6,149.3,146.3,145.0,142.4,141.6,139.6$, $120.1,114.4$, 113.8, 110.9, 110.7, 102.4, 102.2, 60.6, 54.7, 42.4, 34.2, 30.5, 27.5, 19.1, 19.0, 11.8, 11.6 ppm.

IR (ATR): $\tilde{\boldsymbol{v}}=2941,2863,1454,1364,1024,995,919,880,741,674,659,575 \mathrm{~cm}^{-1}$.

HRMS $\left(\mathrm{MALDI}^{+}\right.$) $m / z:[\mathrm{M}]^{++}$: calcd. for $\left[\mathrm{C}_{79} \mathrm{H}_{106} \mathrm{~N}_{8} \mathrm{~S}_{2} \mathrm{Si}_{4}\right]^{++}:$1342.7053; found 1342.7057; correct isotope distribution.

\section{Crystal data}

Single crystalline specimen were obtained by slow diffusion of methanol into a chloroform solution of 3c:

Green crystal (plate), dimensions $0.126 \times 0.111 \times 0.024 \mathrm{~mm}^{3}$, crystal system monoclinic, space group C2/c, $Z=4, \quad a=37.1165(18) \AA, \quad b=13.9744(4) \AA, \quad c=15.4894(6) \AA, \quad \alpha=90^{\circ}, \quad \beta=94.818(4)^{\circ}, \quad \gamma=90^{\circ}$, $V=8005.7(5) \AA^{3}, \rho=1.115 \mathrm{~g} / \mathrm{cm}^{3}, T=200(2) \mathrm{K}, \Theta_{\max }=63.681^{\circ}, 35963$ reflections measured, 6577 unique $\left(\mathrm{R}_{\text {int }}=0.0559\right), 3714$ observed $(\mathrm{I}>2 \sigma(\mathrm{I})), \mu=1.52 \mathrm{~mm}^{-1}, T_{\min }=0.61, T_{\max }=1.63,524$ parameters refined, hydrogen atoms were treated using appropriate riding models, goodness of fit 1.04 for observed reflections, final residual values $\mathrm{R} 1(\mathrm{~F})=0.071, \mathrm{wR}\left(\mathrm{F}^{2}\right)=0.194$ for observed reflections, residual electron density -0.39 to $0.31 \mathrm{e}^{-3}$. 


\section{NMR Spectra}

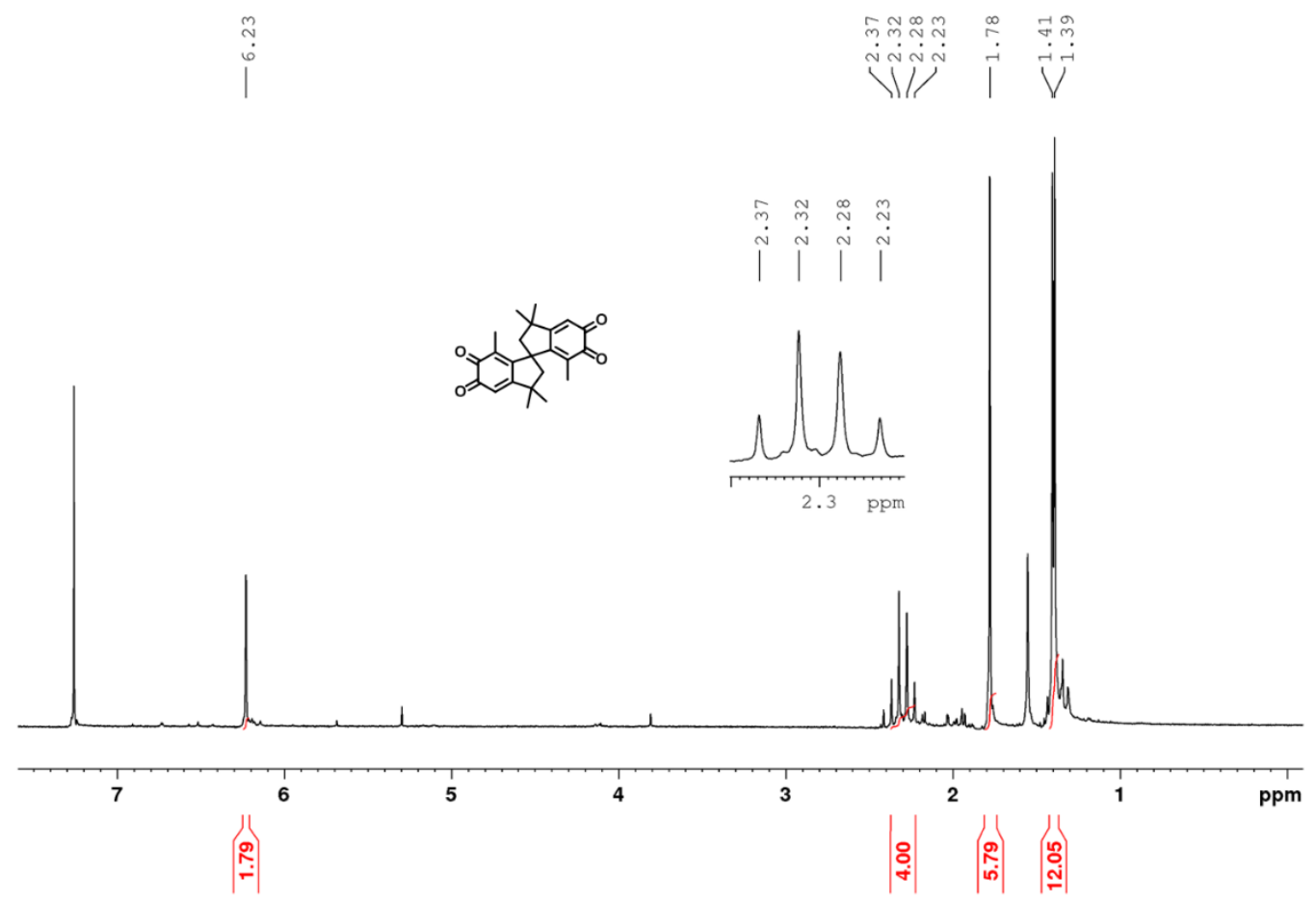

Figure S1: ${ }^{1} \mathrm{H} N M R$ spectrum (300 $\left.\mathrm{MHz}\right)$ of 4 in $\mathrm{CDCl}_{3}$.
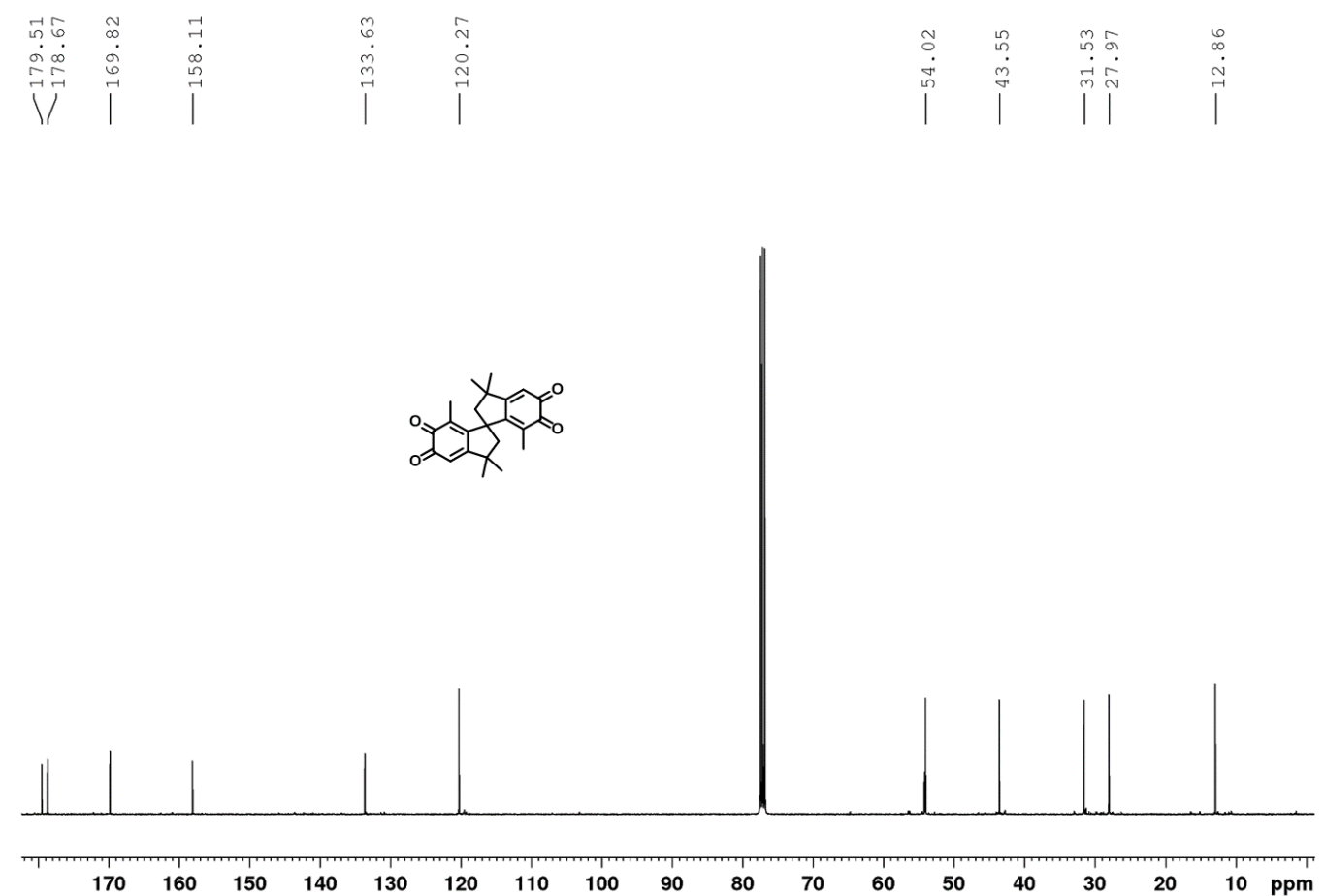

Figure S2: $\left.{ }^{13} C_{\{}{ }^{1} \mathrm{H}\right\}$ NMR spectrum $(101 \mathrm{MHz})$ of 4 in $\mathrm{CDCl}_{3}$. 

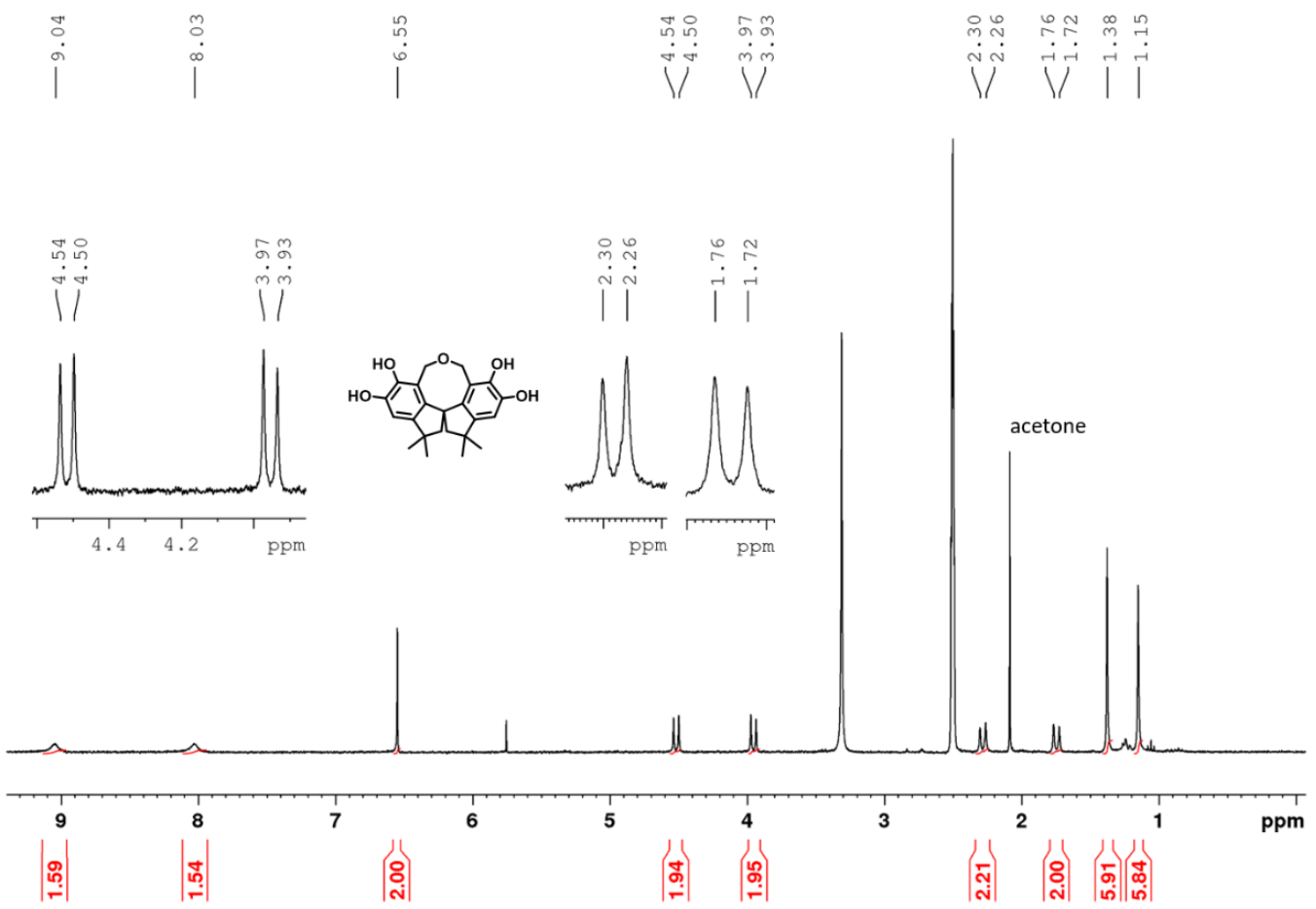

Figure S3: ${ }^{1} H$ NMR spectrum (300 MHz) of $\mathbf{S 5}$ in DMSO-d .

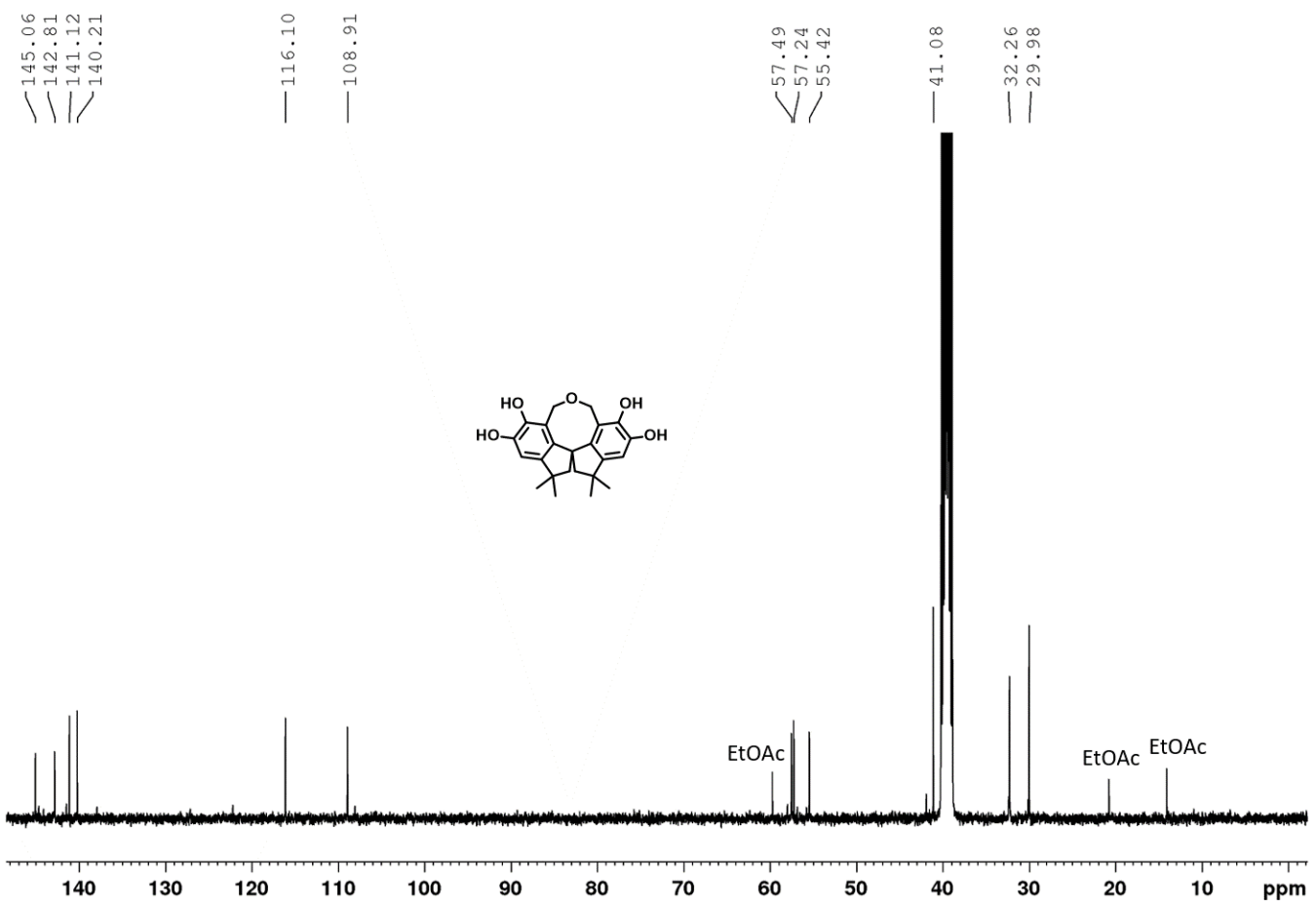

Figure S4: $\left.{ }^{13} C_{\{}{ }^{1} H\right\}$ NMR spectrum (101 MHz) of $\boldsymbol{S 5}$ in DMSO-d6. 


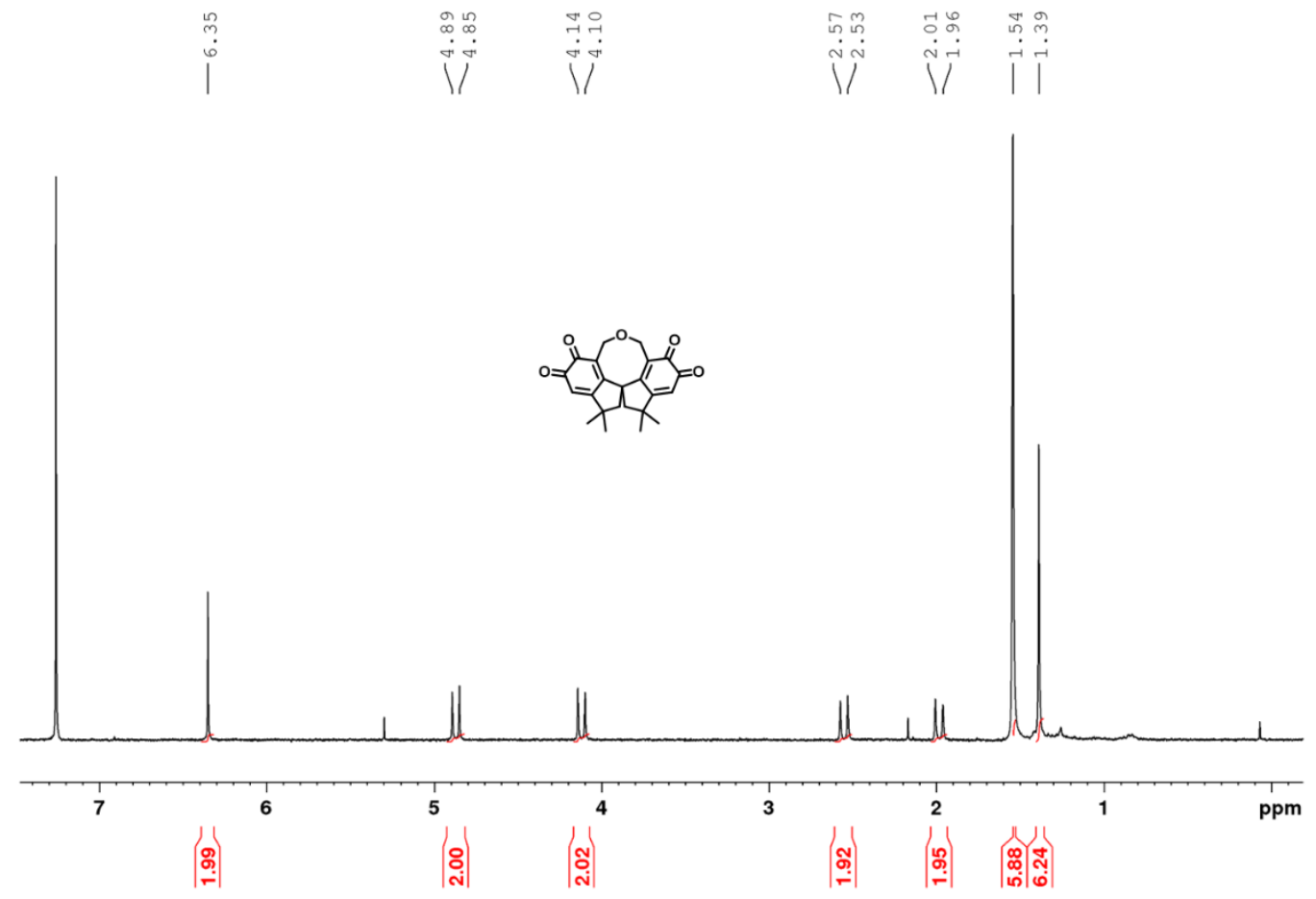

Figure S5: ${ }^{1} \mathrm{H} N \mathrm{NR}$ spectrum $(300 \mathrm{MHz})$ of 5 in $\mathrm{CDCl}_{3}$.

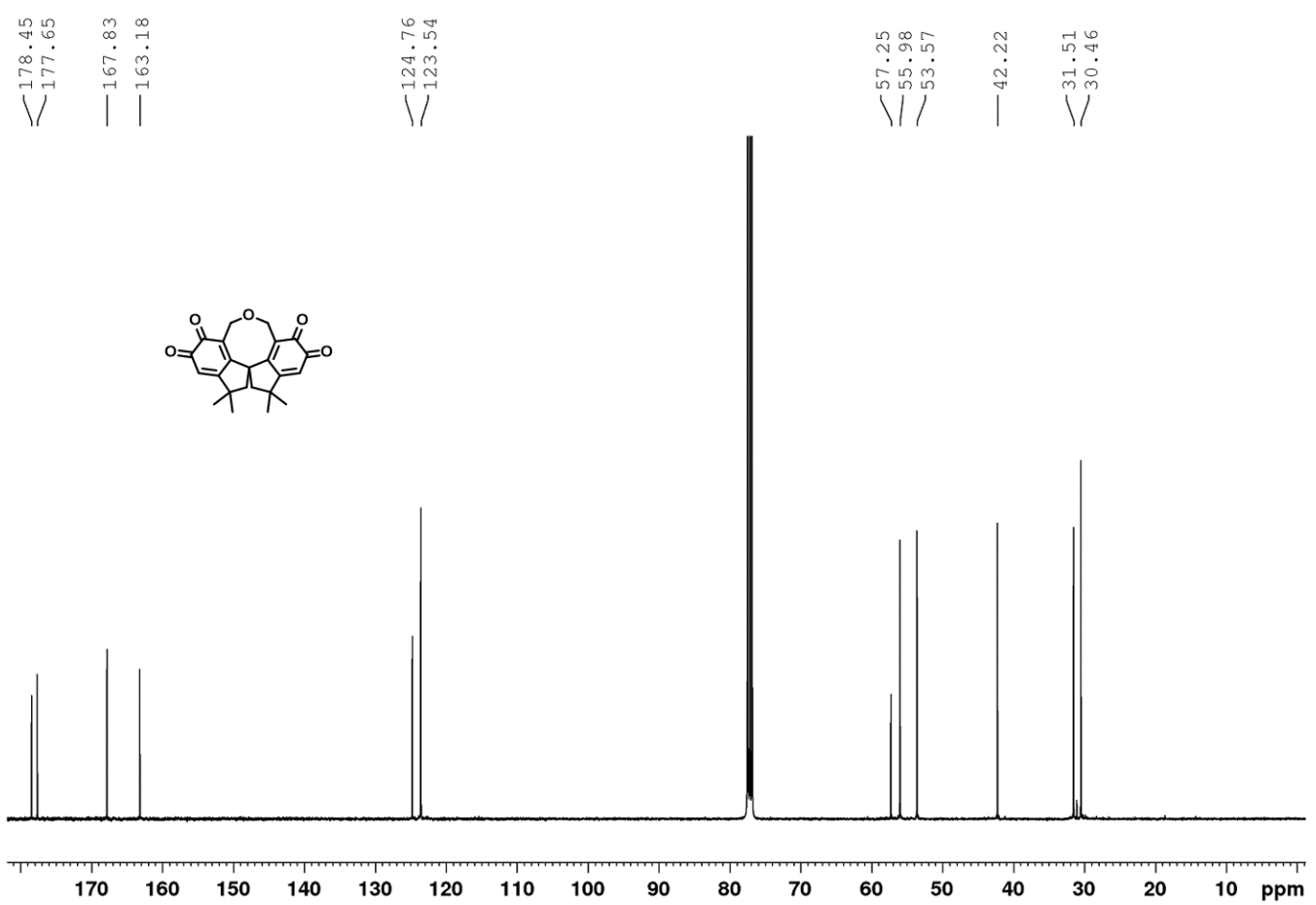

Figure S6: ${ }^{13} C_{\left\{{ }^{1} \mathrm{H}\right\}} \mathrm{NMR}$ spectrum $(101 \mathrm{MHz})$ of 5 in $\mathrm{CDCl}_{3}$. 


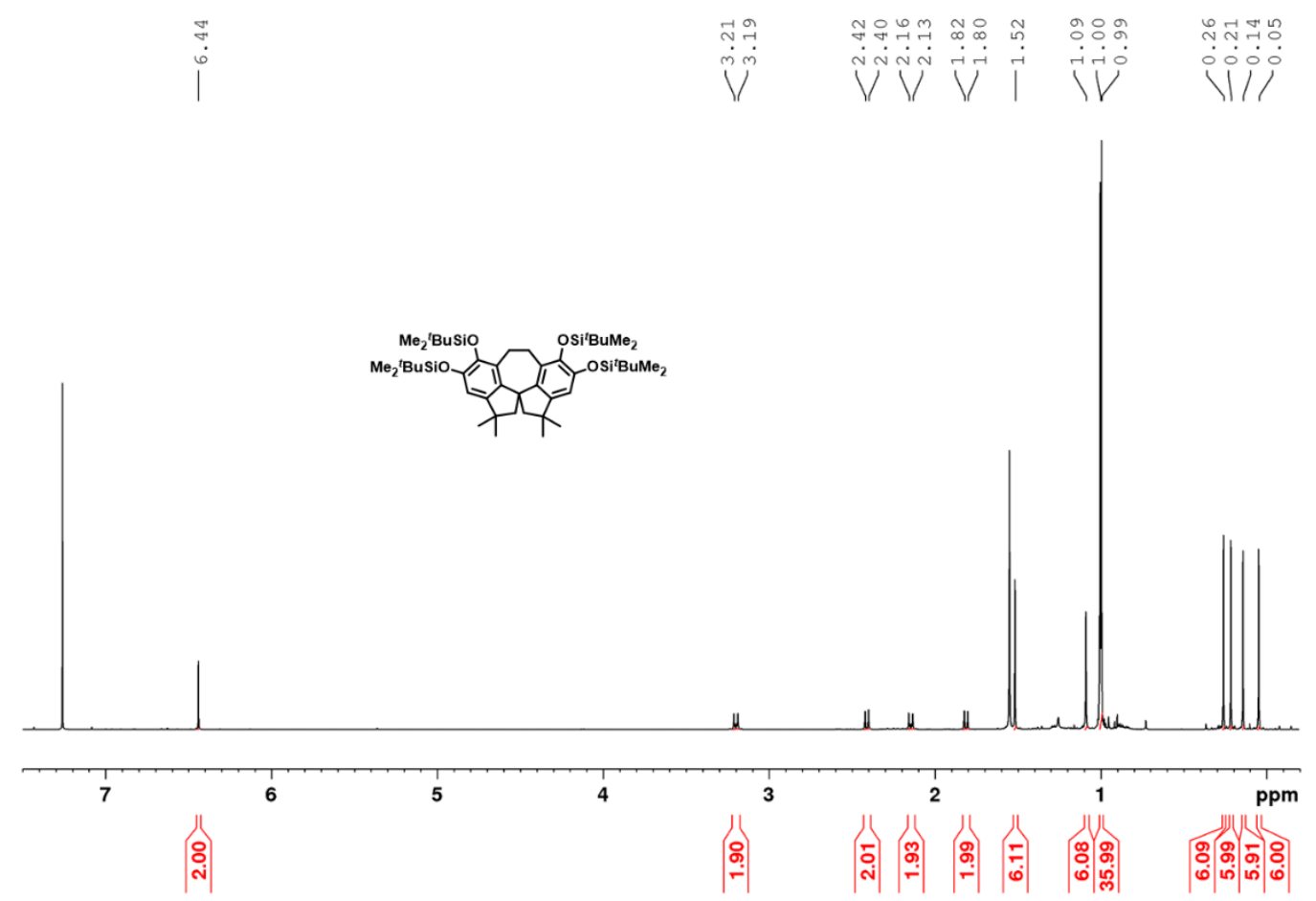

Figure S7: ${ }^{1} \mathrm{H} N \mathrm{MR}$ spectrum $(600 \mathrm{MHz})$ of $\mathbf{S 6}$ in $\mathrm{CDCl}_{3}$.

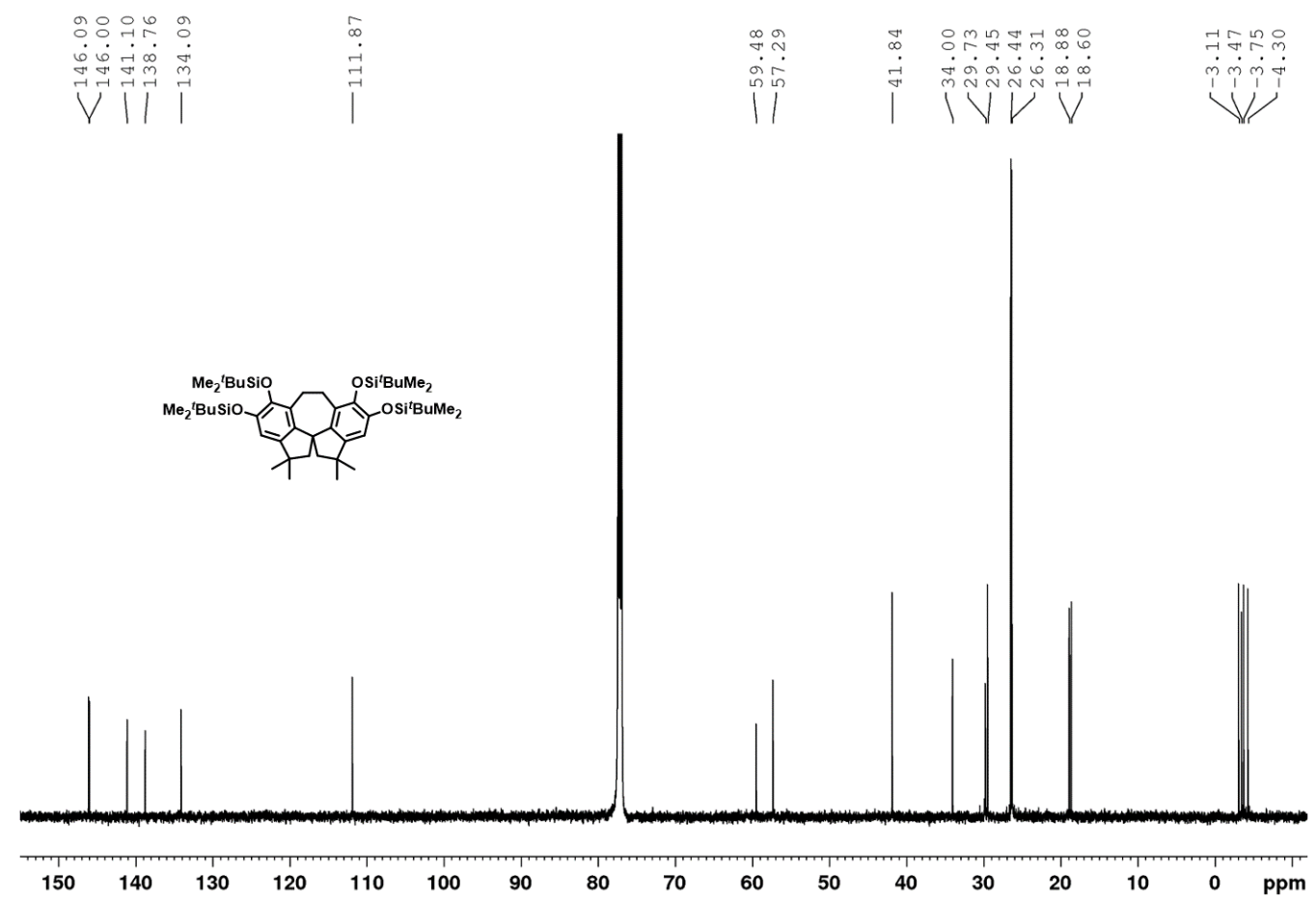

Figure S8: $\left.{ }^{13} C_{\{1}^{1} \mathrm{H}\right\}$ NMR spectrum $(151 \mathrm{MHz})$ of $\mathbf{S 6}$ in $\mathrm{CDCl}_{3}$. 


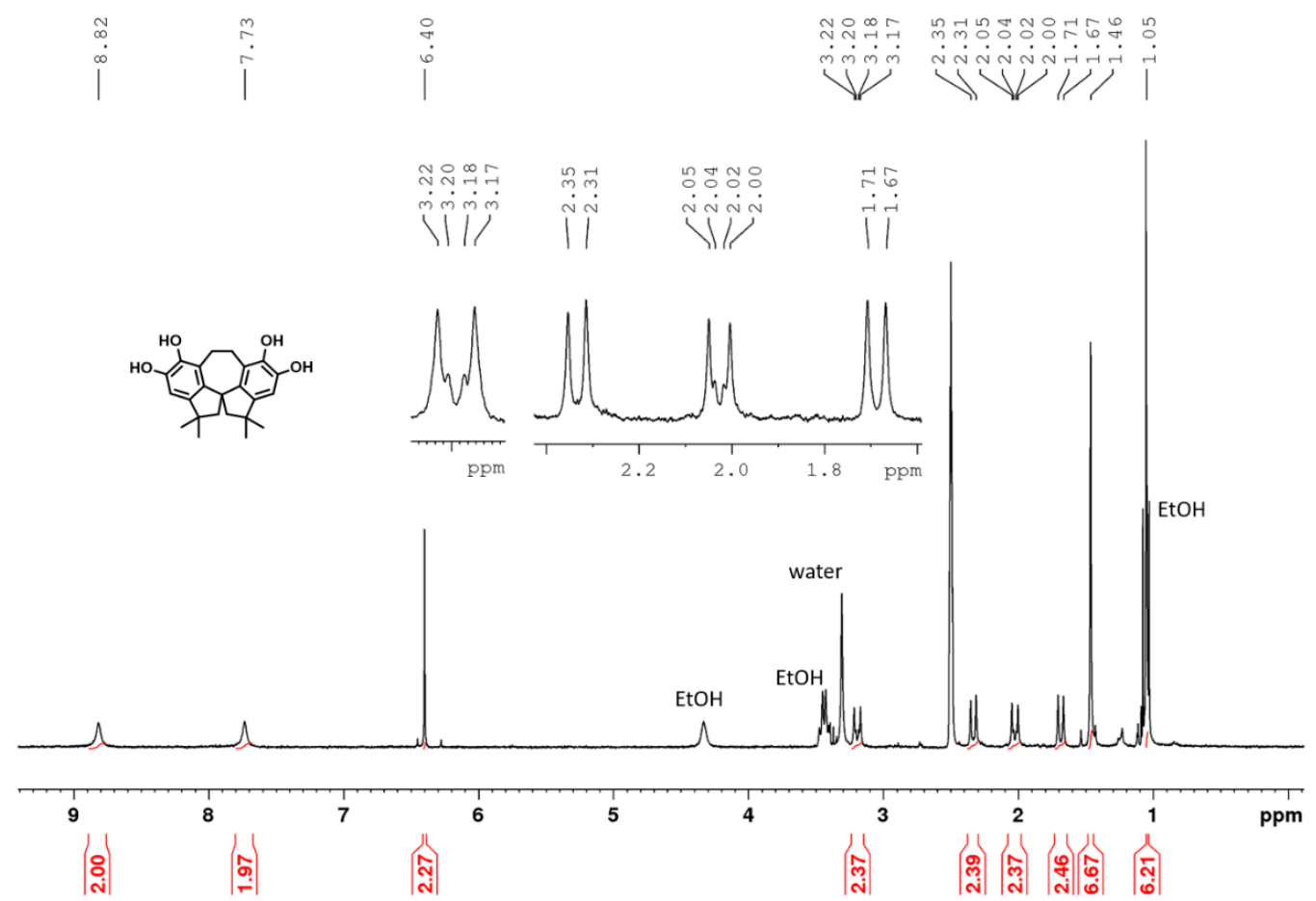

Figure S9: ${ }^{1} \mathrm{H} N M R$ spectrum $(300 \mathrm{MHz})$ of $\mathbf{S} 7$ in DMSO-d6.

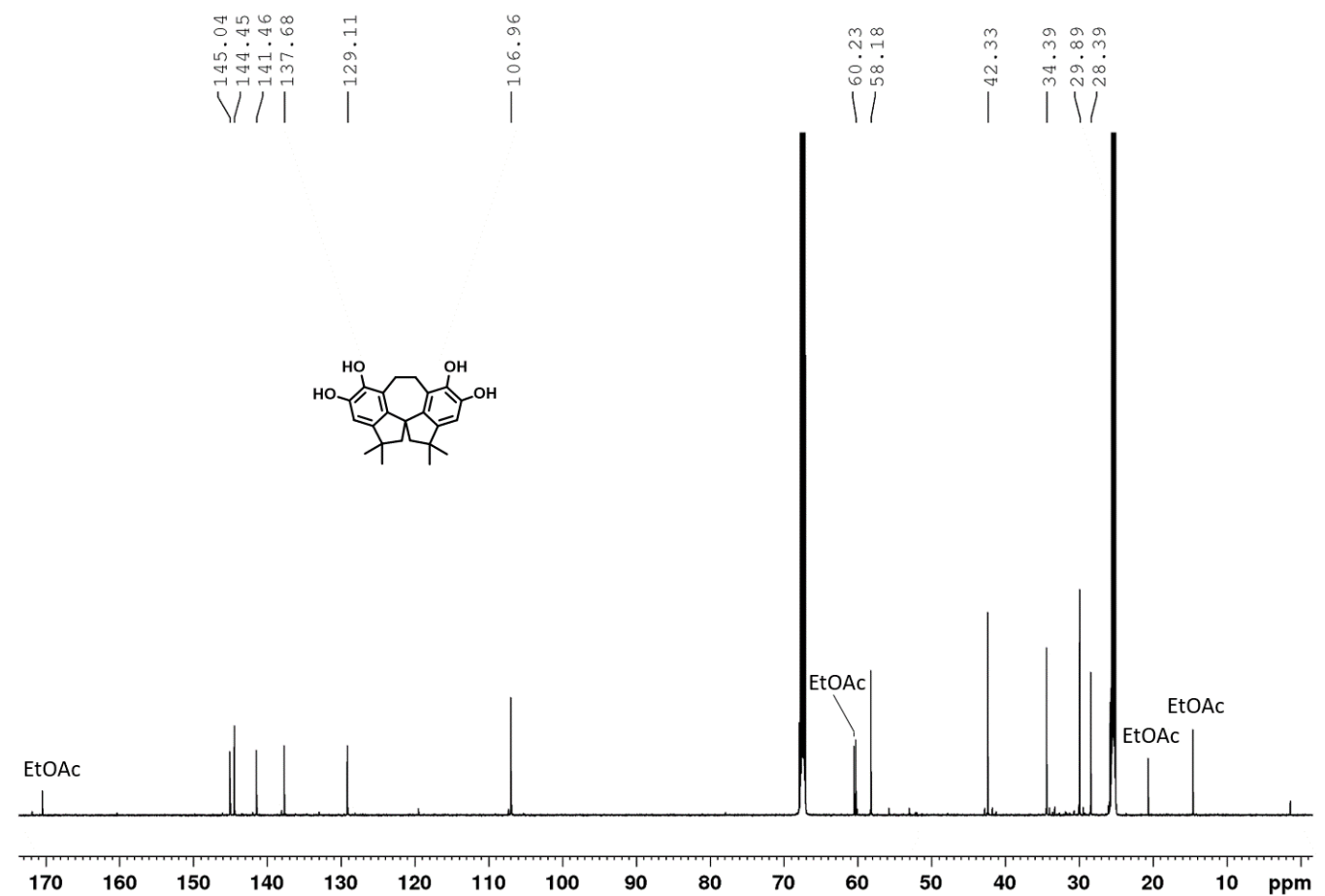

Figure S10: ${ }^{13} C_{\left\{{ }^{1} H\right\}} \mathrm{NMR}$ spectrum $(151 \mathrm{MHz})$ of $\boldsymbol{S} 7$ in $\mathrm{THF}-\mathrm{d}_{8}$. 


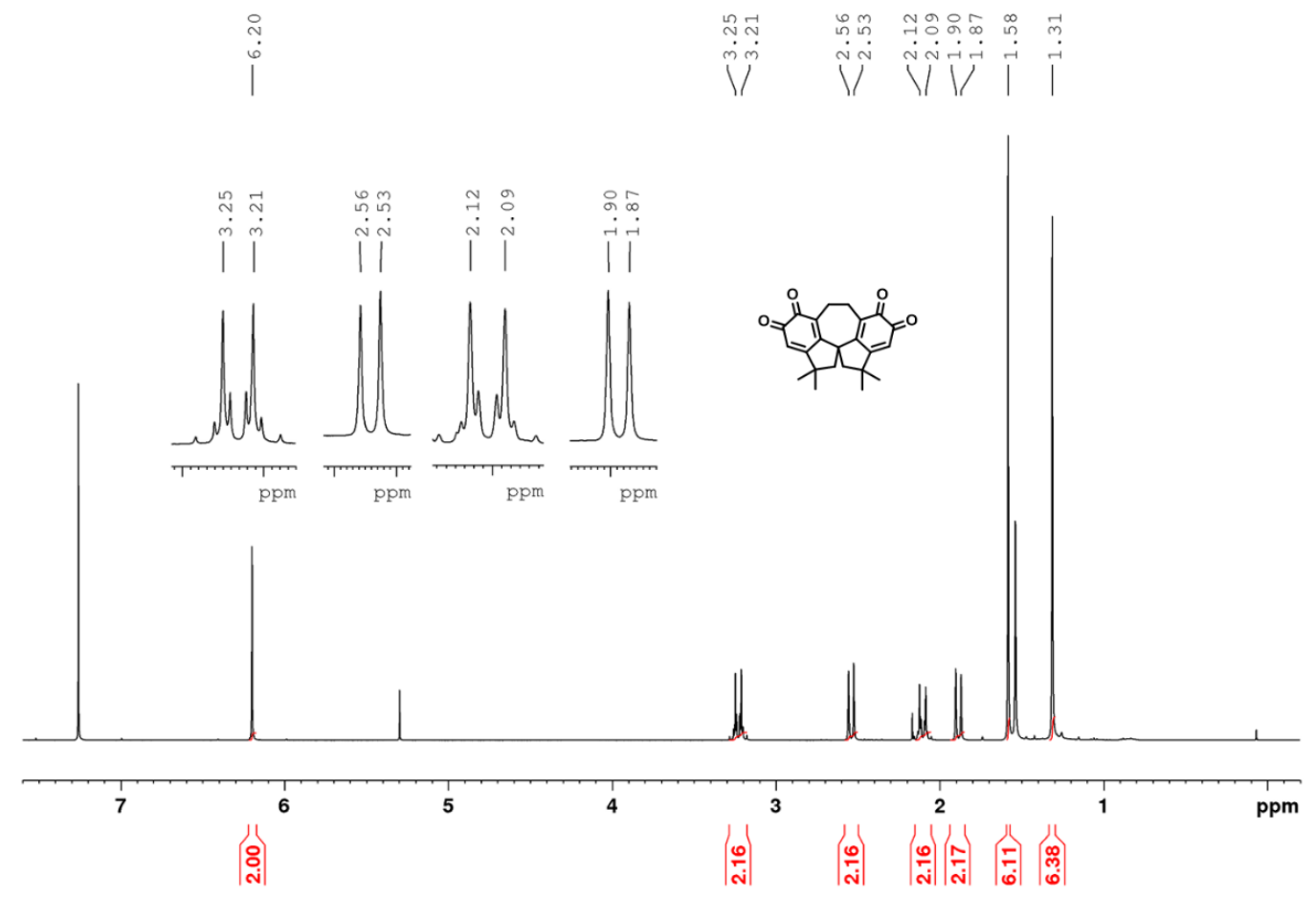

Figure S11: ${ }^{1} \mathrm{H} N \mathrm{MR}$ spectrum $(400 \mathrm{MHz})$ of 6 in $\mathrm{CDCl}_{3}$.

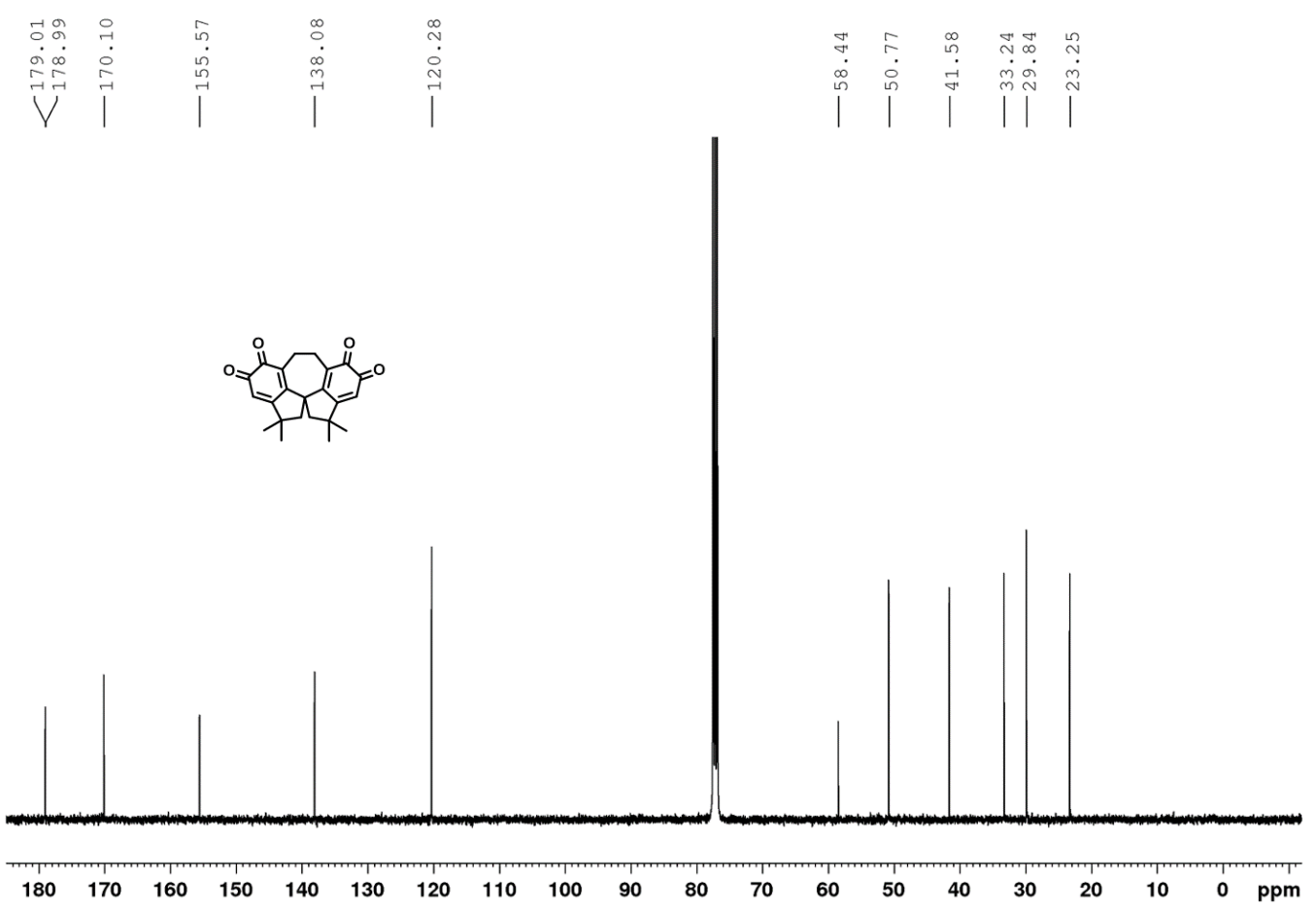

Figure S12: ${ }^{13} \mathrm{C}_{\left\{{ }^{1} \mathrm{H}\right\}} \mathrm{NMR}$ spectrum (101 MHz) of $\mathbf{6}$ in $\mathrm{CDCl}_{3}$. 


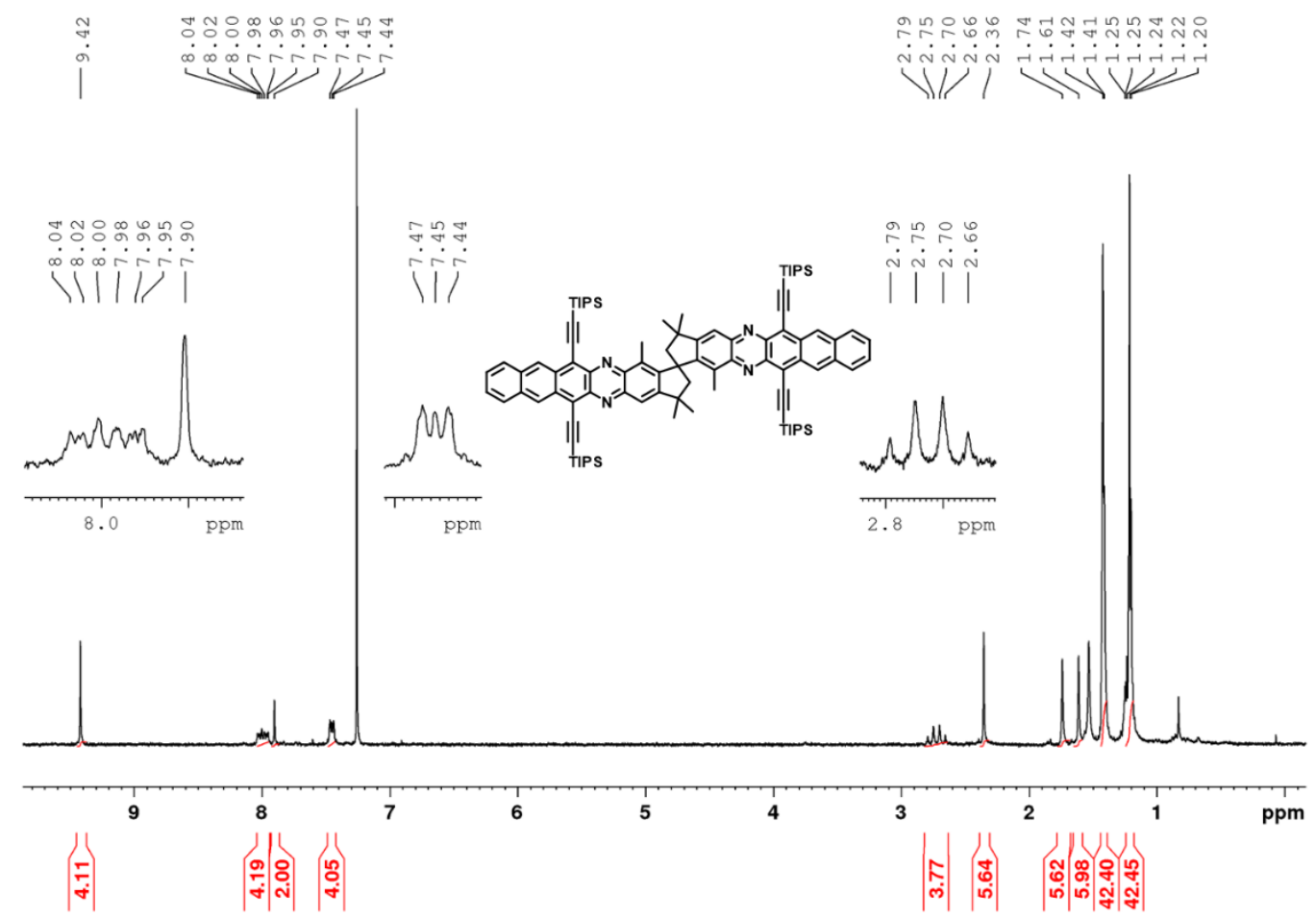

Figure S13: ${ }^{1} \mathrm{H} N \mathrm{NR}$ spectrum $(300 \mathrm{MHz})$ of 1 a in $\mathrm{CDCl}_{3}$.

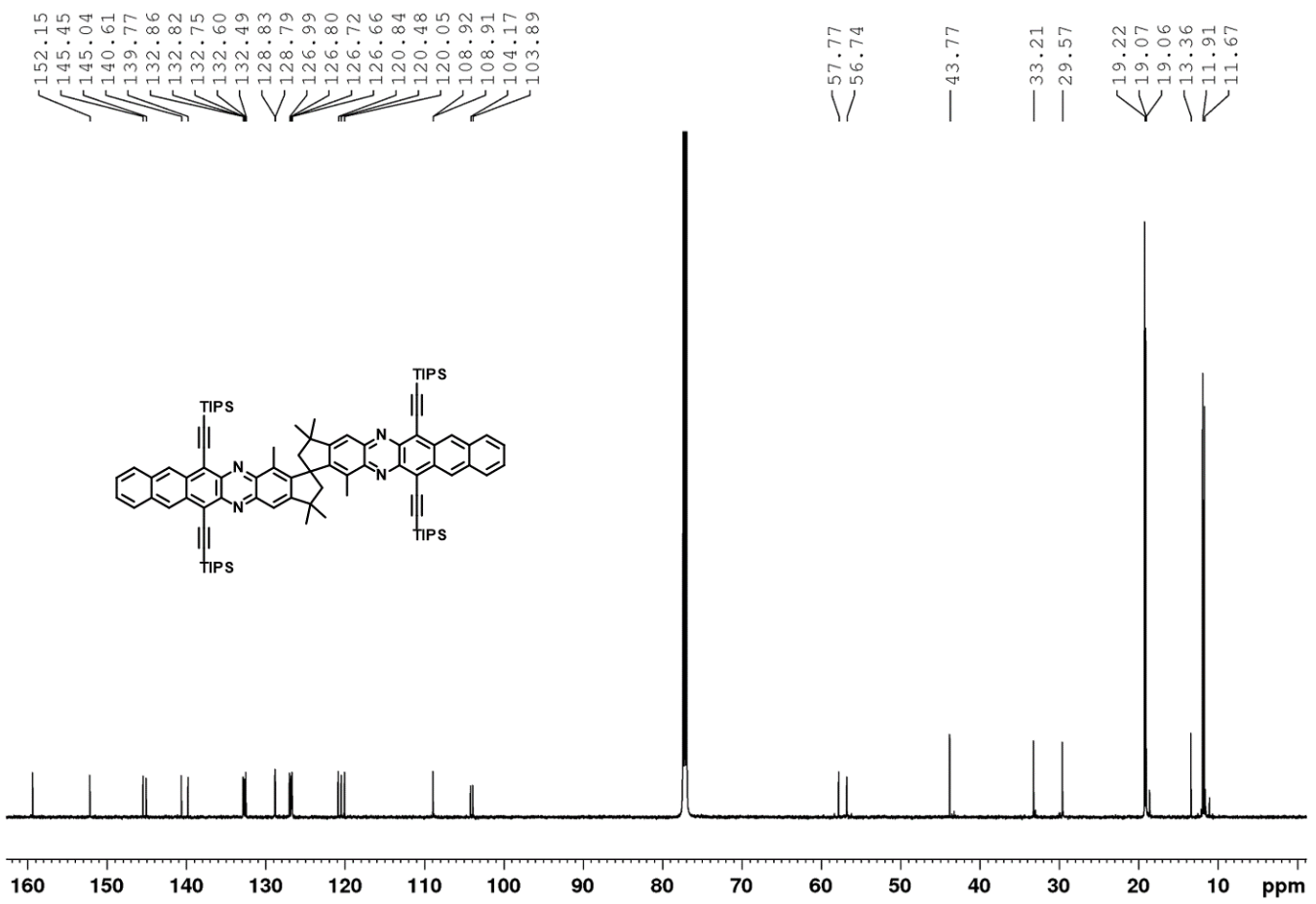

Figure S14: $\left.{ }^{13} C_{\{}^{\{1} H\right\}$ NMR spectrum $(151 \mathrm{MHz})$ of $\mathbf{1 a}$ in $\mathrm{CDCl}_{3}$. 


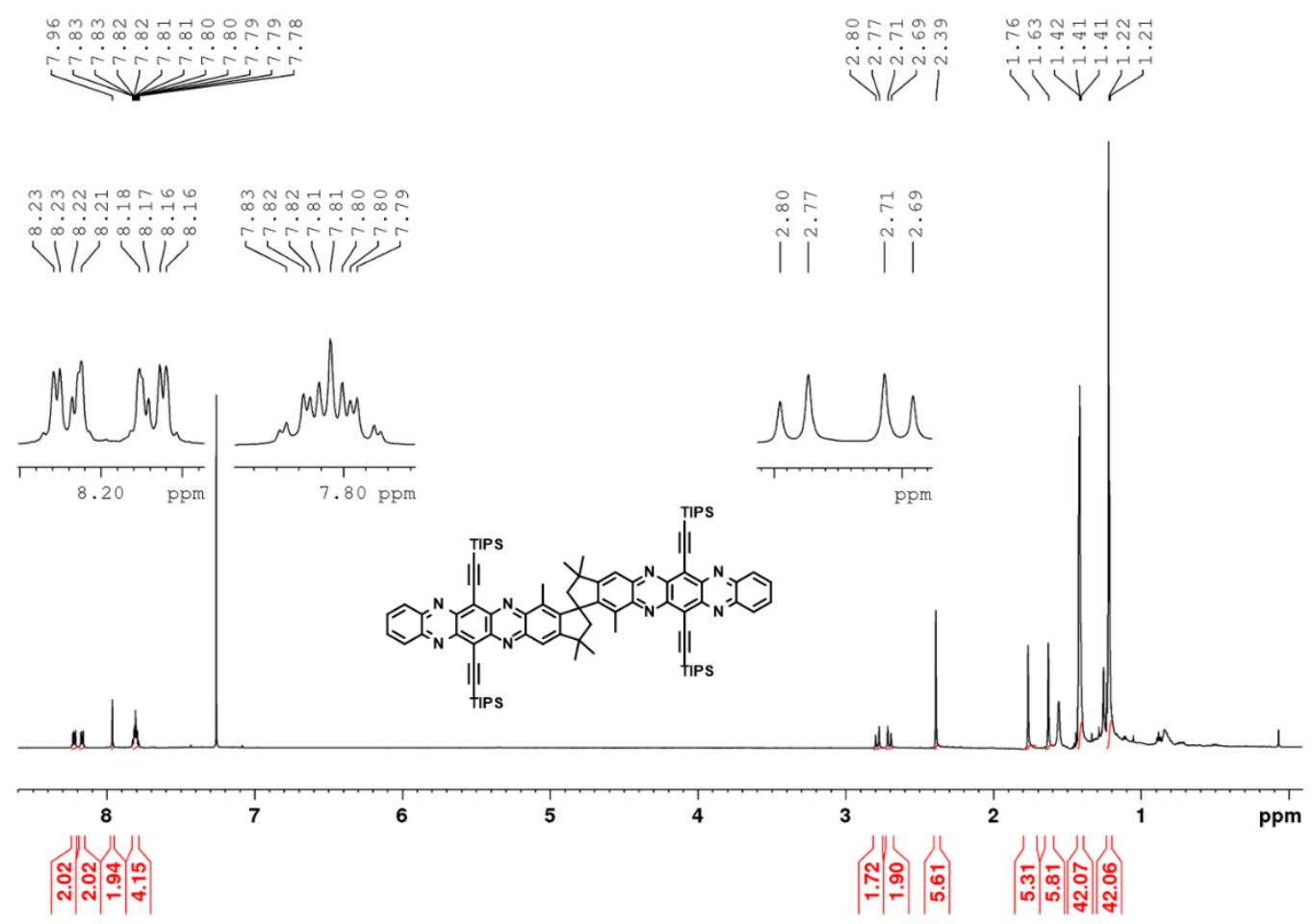

Figure S15: ${ }^{1} \mathrm{H} N \mathrm{MR}$ spectrum $(600 \mathrm{MHz})$ of $\mathbf{1 b}$ in $\mathrm{CDCl}_{3}$.

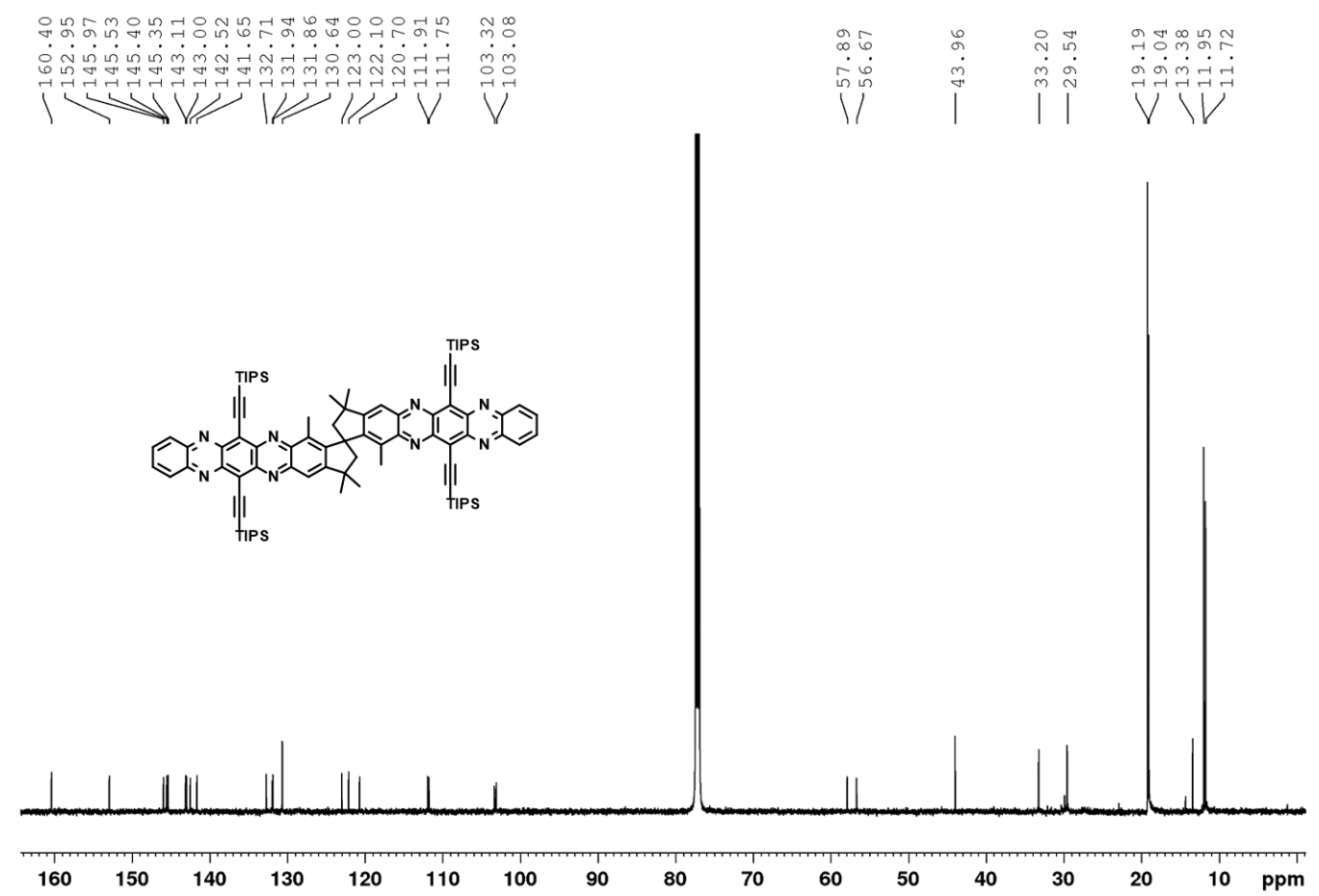

Figure S16: $\left.{ }^{13} C_{\{}^{\{1} H\right\}$ NMR spectrum $(151 \mathrm{MHz})$ of $\mathbf{1 b}$ in $\mathrm{CDCl}_{3}$. 


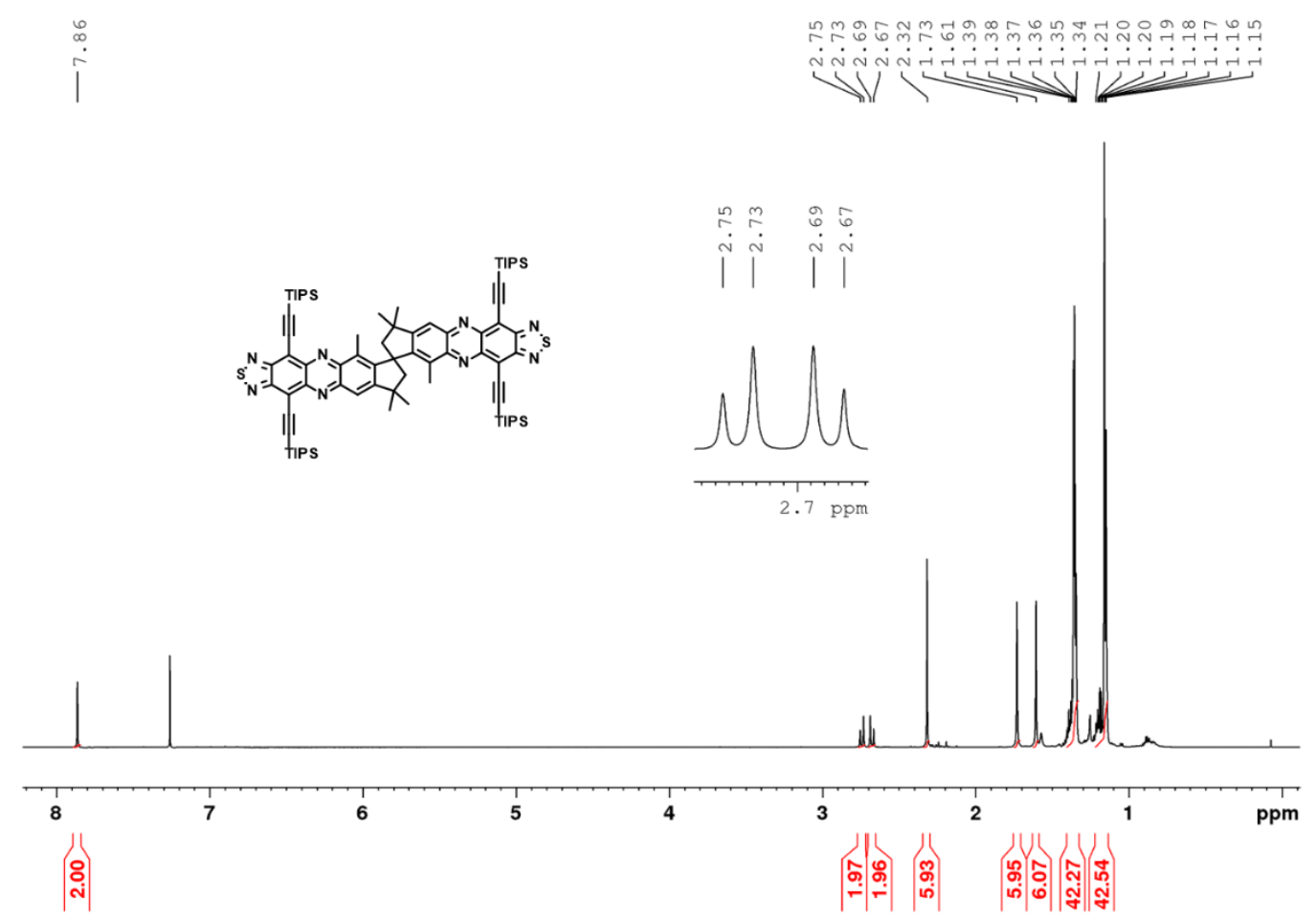

Figure S17: ${ }^{1} \mathrm{H} N \mathrm{MR}$ spectrum $(600 \mathrm{MHz})$ of $1 \mathrm{c}$ in $\mathrm{CDCl}_{3}$.

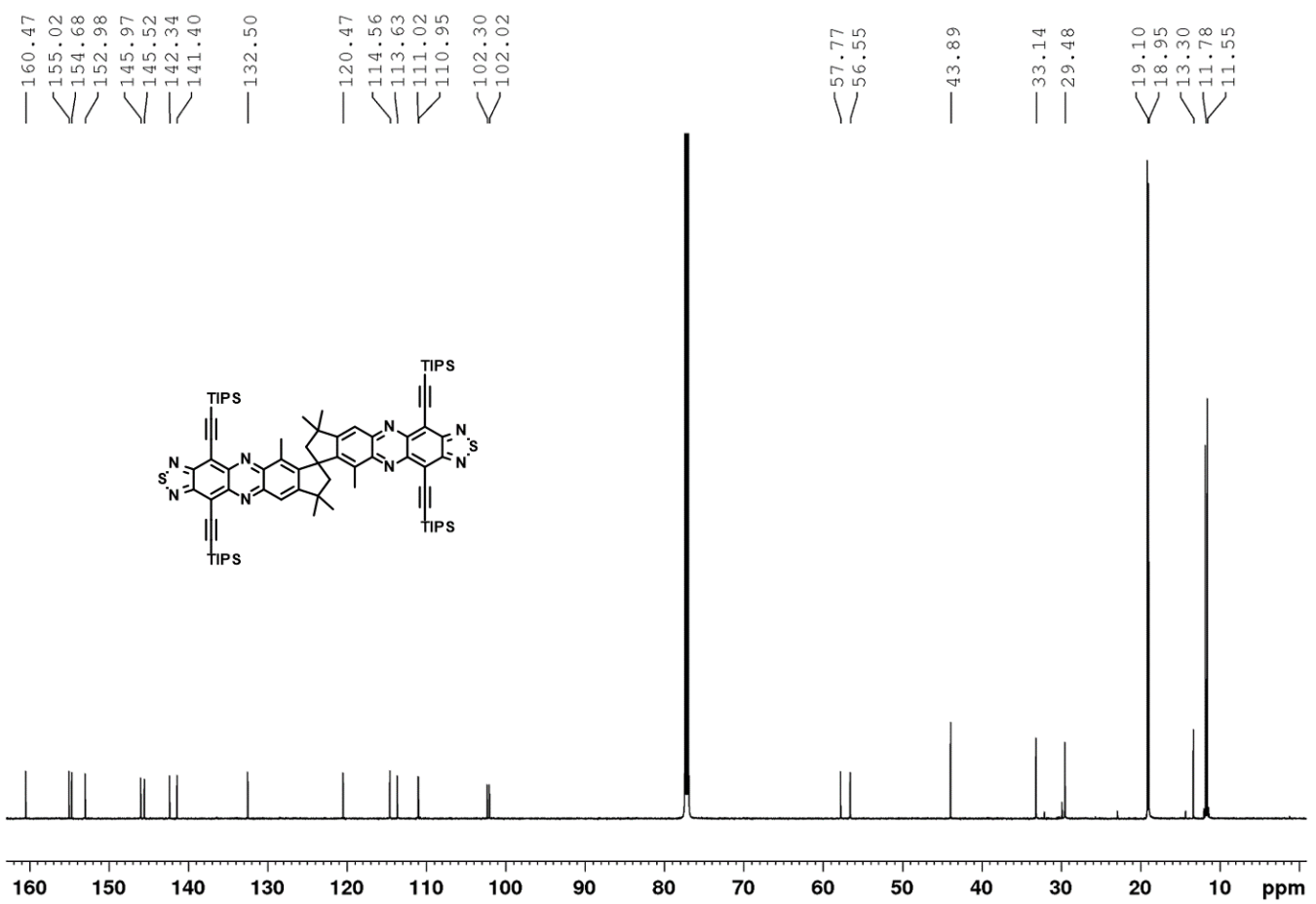

Figure S18: $\left.{ }^{13} C_{\{}^{\{1} H\right\}$ NMR spectrum $(151 \mathrm{MHz})$ of $1 \mathrm{c}$ in $\mathrm{CDCl}_{3}$. 


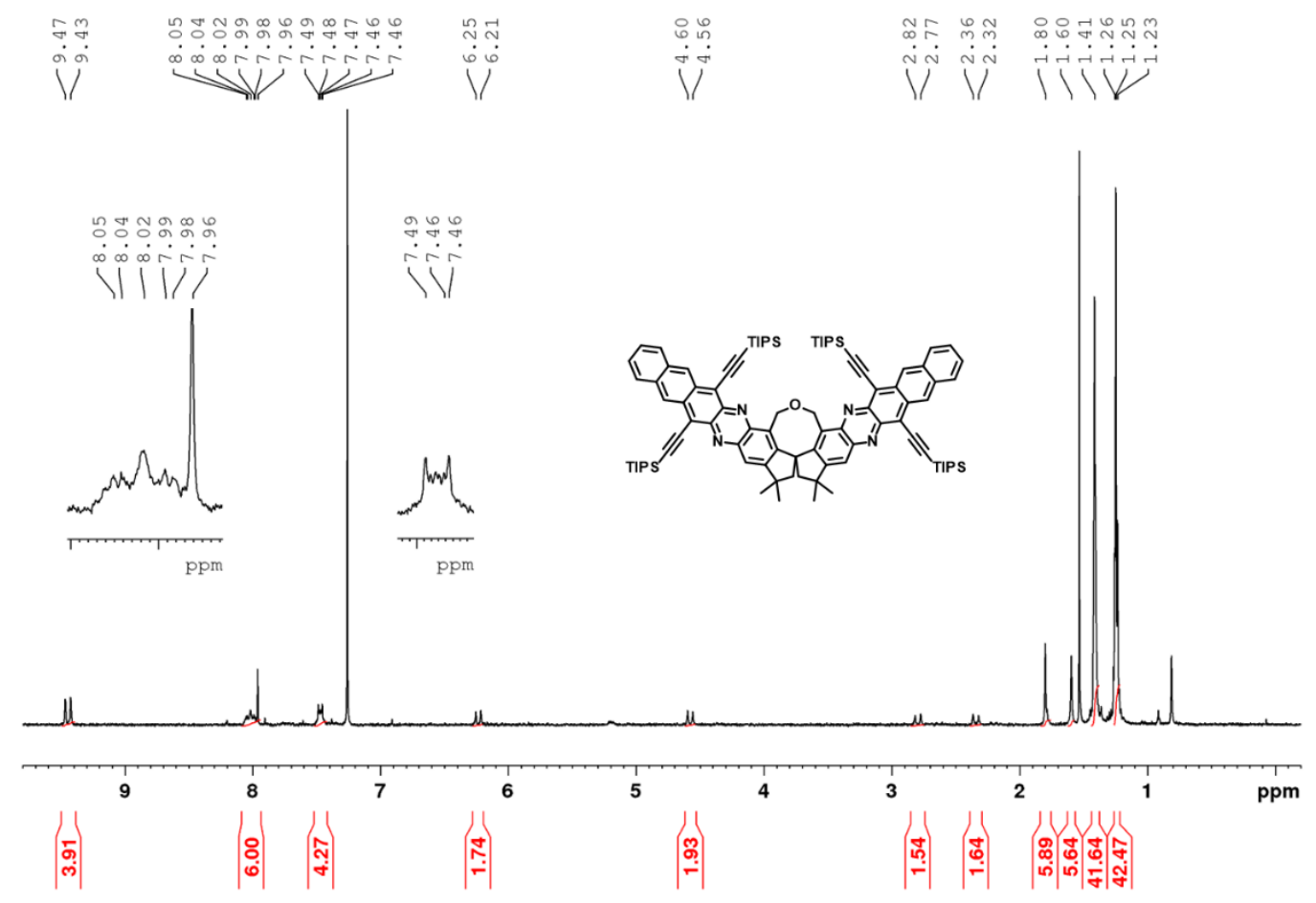

Figure S19: ${ }^{1} \mathrm{H}$ NMR spectrum (300 $\mathrm{MHz}$ ) of 2 a in $\mathrm{CDCl}_{3}$.

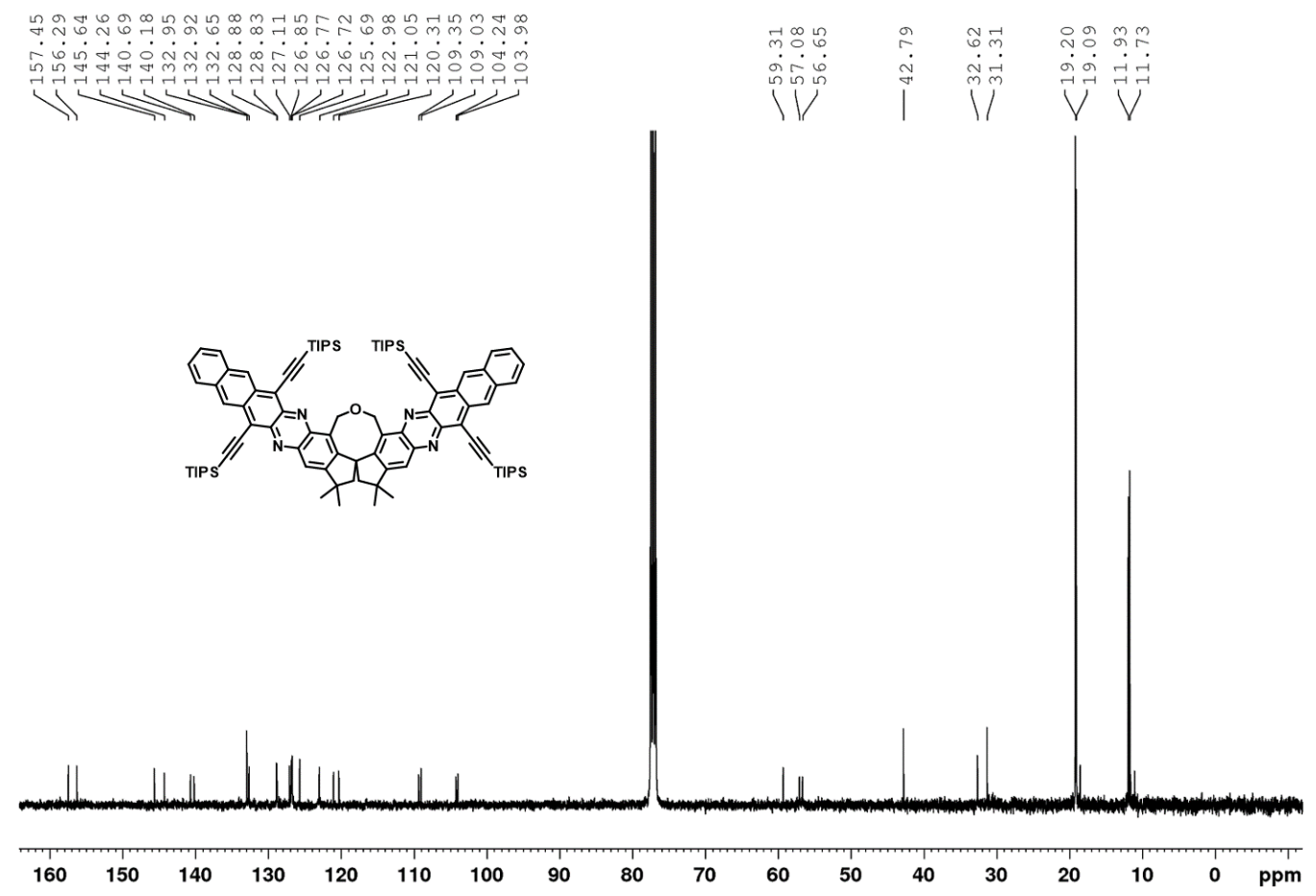

Figure S20: $\left.{ }^{13} C_{\{}{ }^{1} H\right\} N M R$ spectrum $(101 \mathrm{MHz})$ of $\mathbf{2 a}$ in $\mathrm{CDCl}_{3}$. 


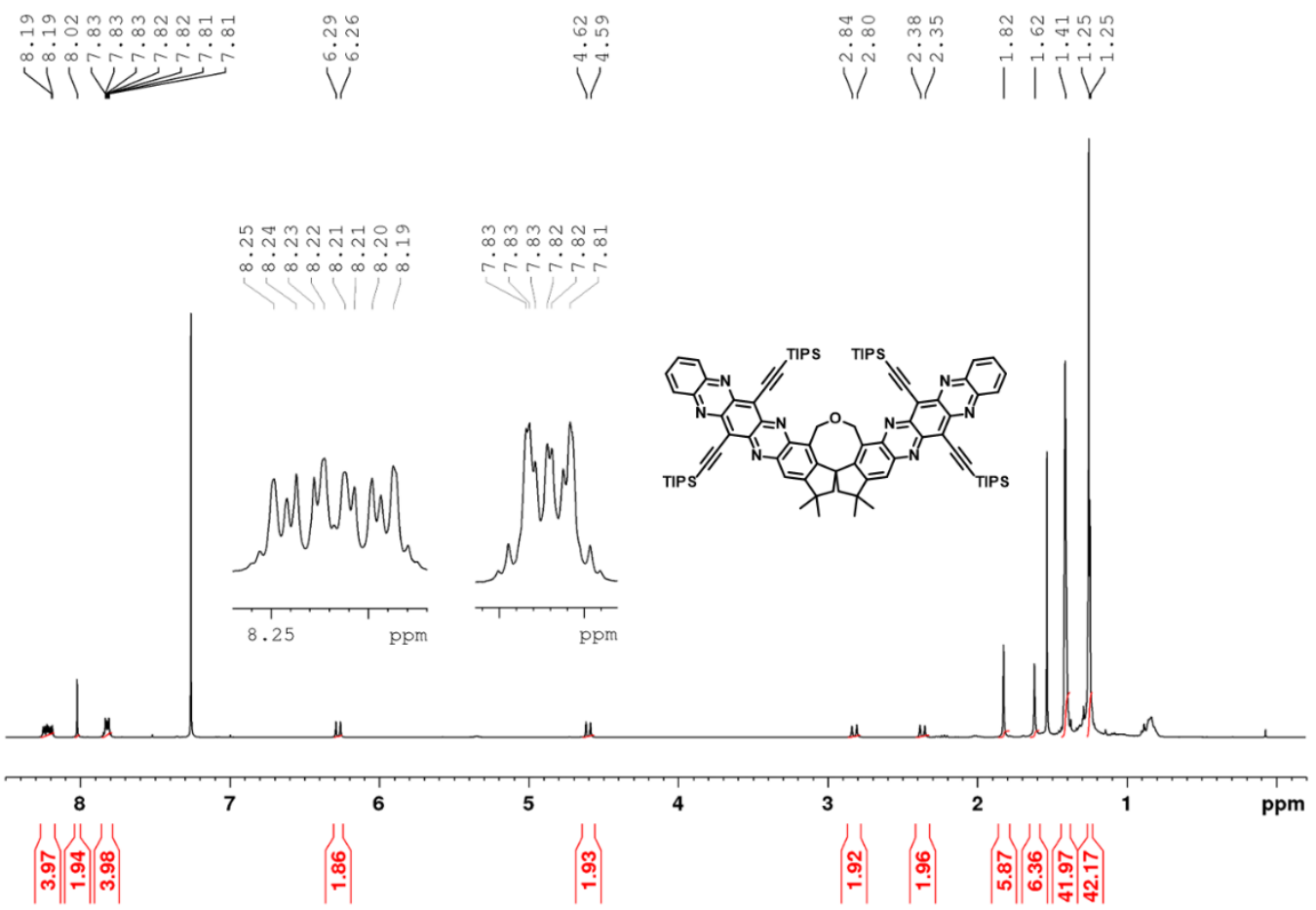

Figure S21: ${ }^{1} \mathrm{H} N \mathrm{NR}$ spectrum $(400 \mathrm{MHz})$ of $\mathbf{2} \boldsymbol{b}$ in $\mathrm{CDCl}_{3}$.

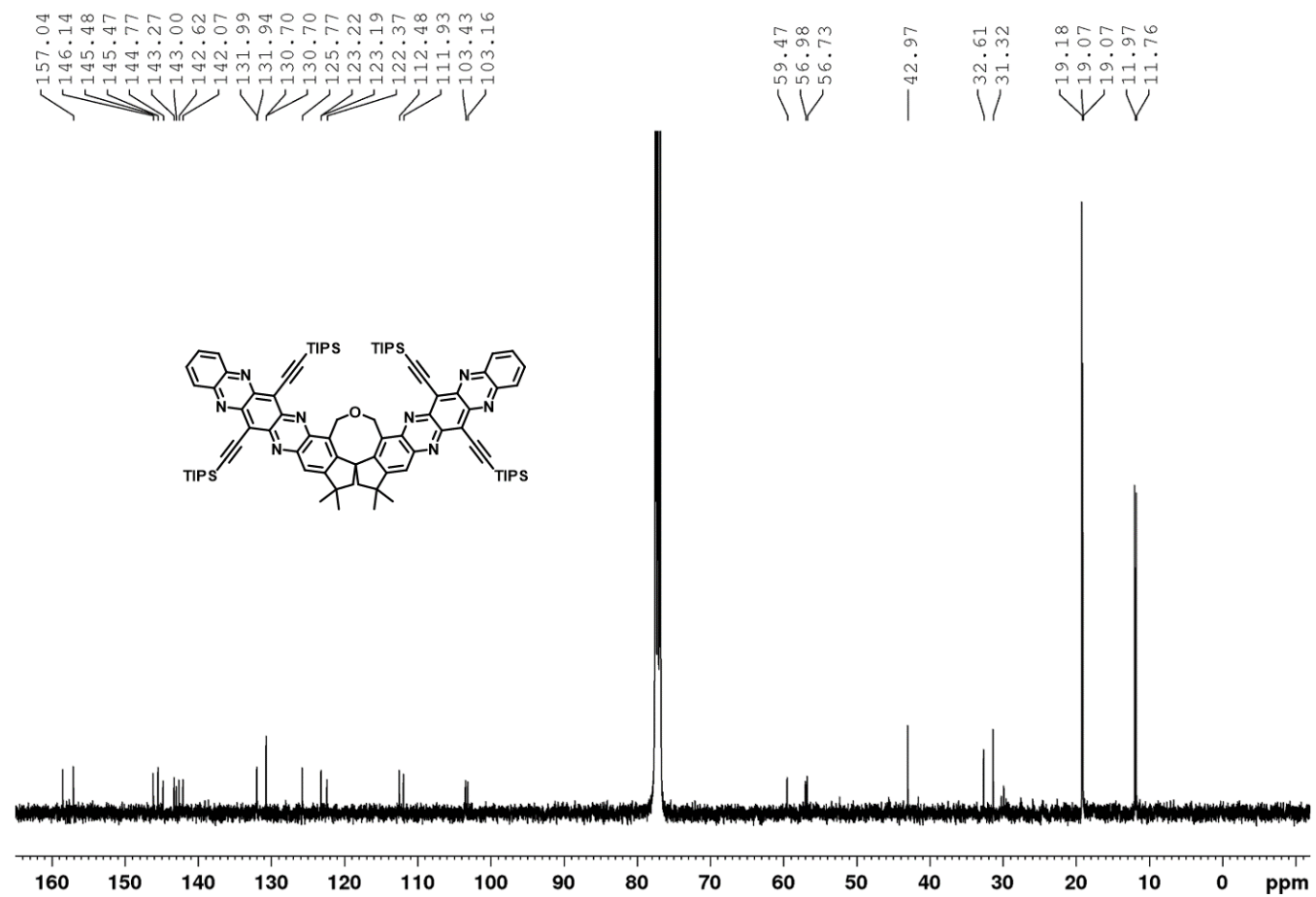

Figure S22: $\left.{ }^{13} C_{\{}{ }^{1} \mathrm{H}\right\} \mathrm{NMR}$ spectrum $(101 \mathrm{MHz})$ of $\mathbf{2 b}$ in $\mathrm{CDCl}_{3}$. 


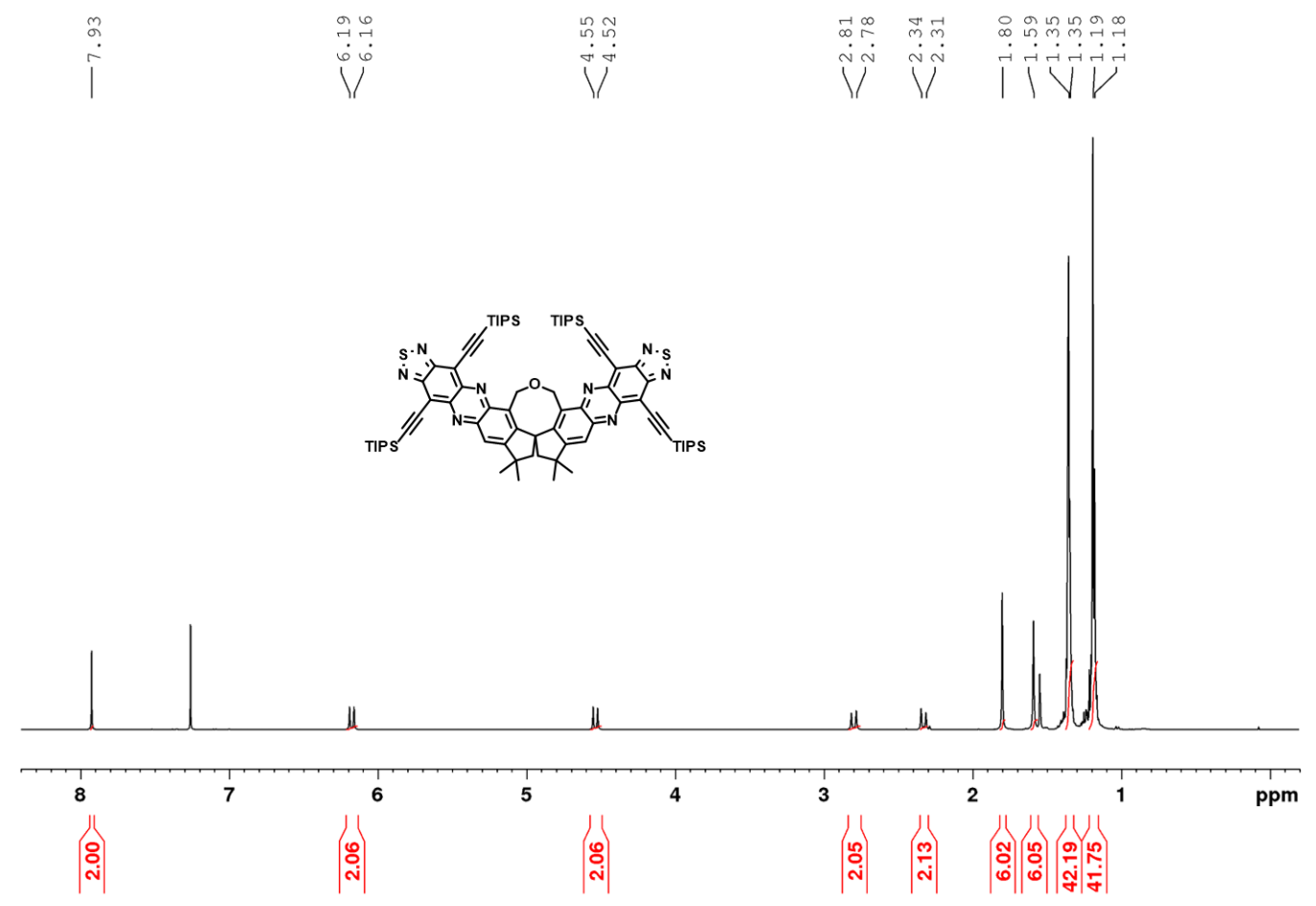

Figure S23: ${ }^{1} \mathrm{H} N \mathrm{MR}$ spectrum $(400 \mathrm{MHz})$ of $2 \mathrm{c}$ in $\mathrm{CDCl}_{3}$.

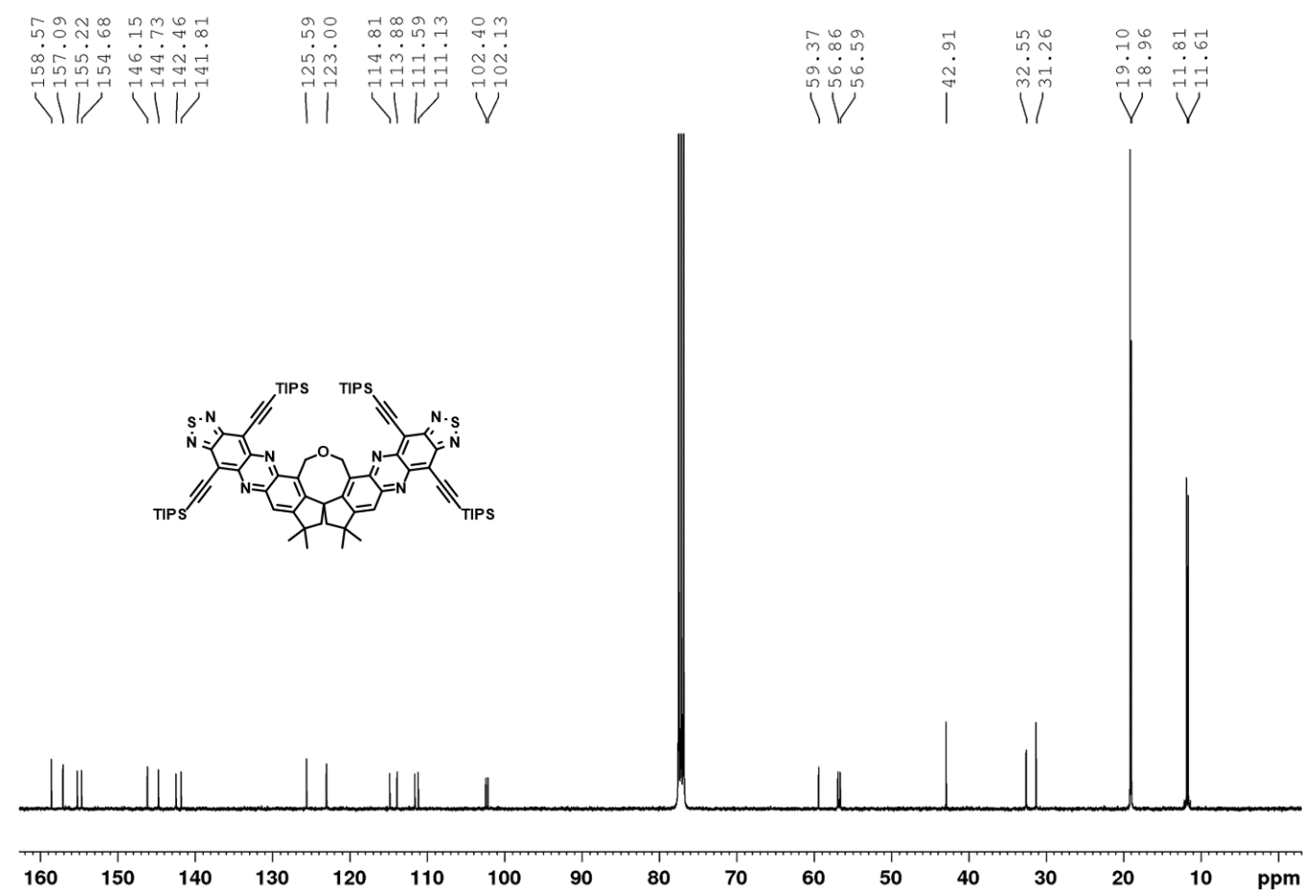

Figure S24: ${ }^{13} C_{\left\{{ }^{1} H\right\}} \mathrm{NMR}$ spectrum $(101 \mathrm{MHz})$ of $2 \mathrm{c}$ in $\mathrm{CDCl}_{3}$. 

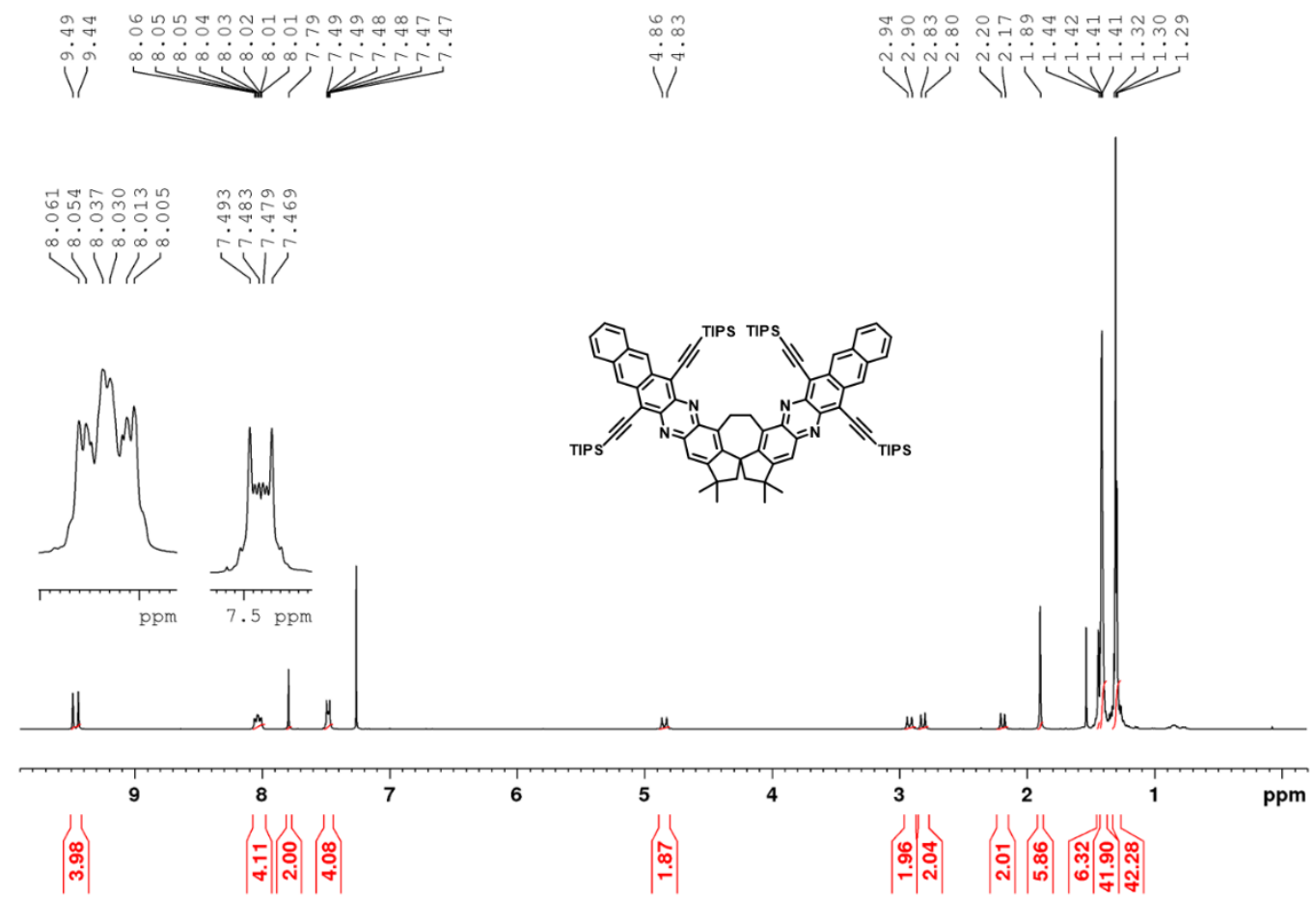

Figure S25: ${ }^{l} \mathrm{H} N \mathrm{NR}$ spectrum $\left(400 \mathrm{MHz}\right.$ ) of 3 a in $\mathrm{CDCl}_{3}$.

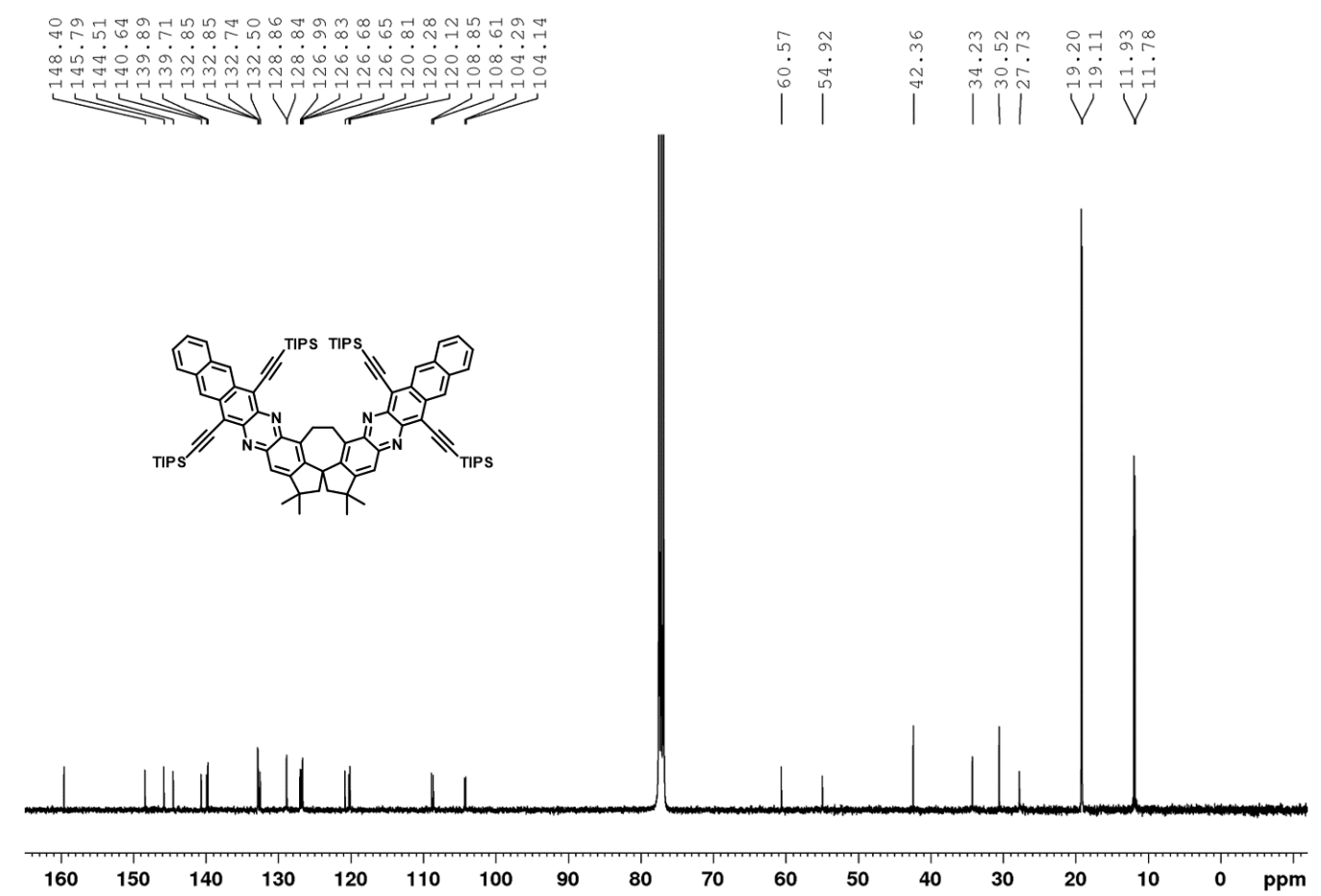

Figure S26: $\left.{ }^{13} C_{\{}{ }^{l} H\right\}$ NMR spectrum (101 MHz) of 3 a in $\mathrm{CDCl}_{3}$. 


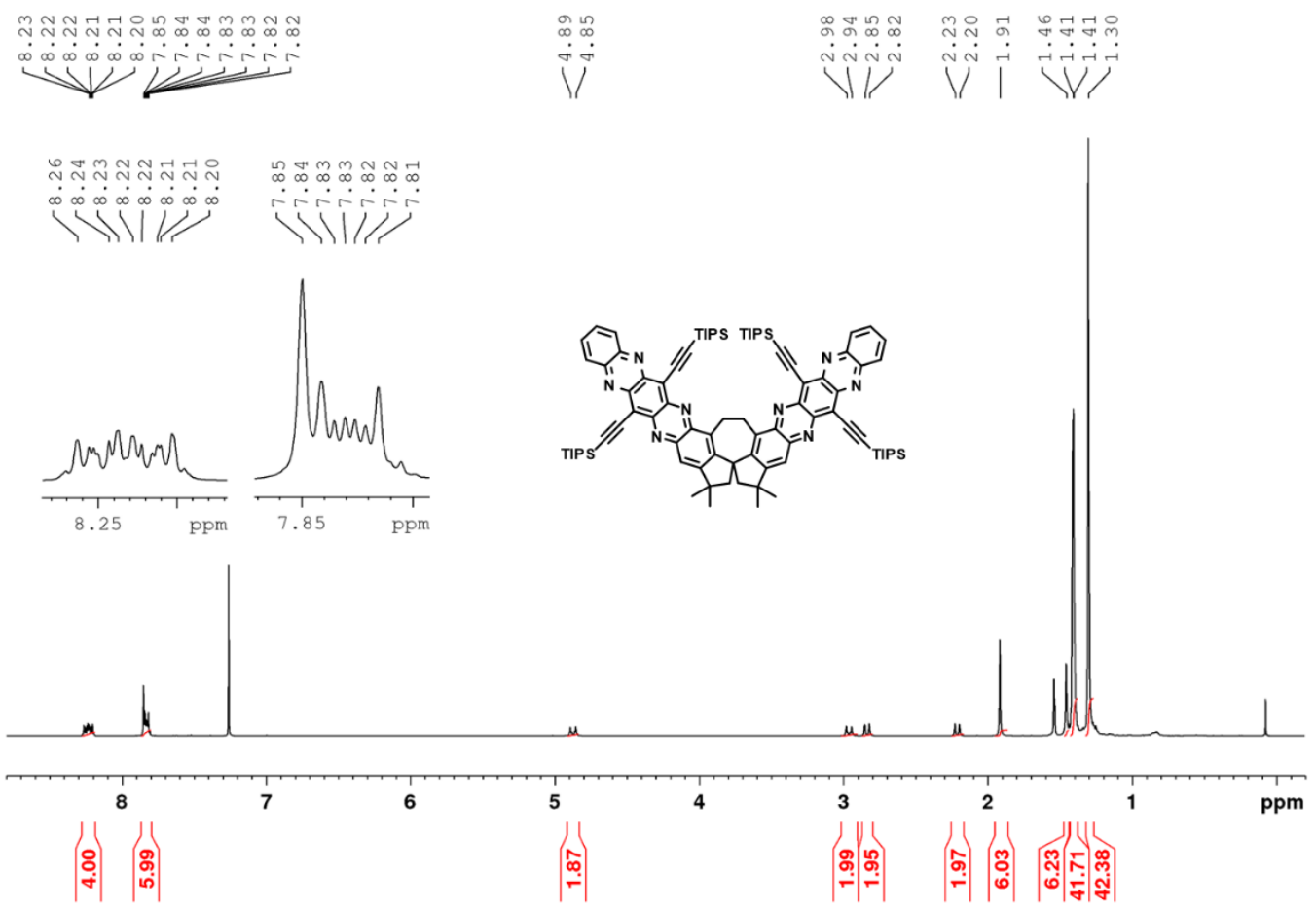

Figure S27: ${ }^{1} \mathrm{H} N \mathrm{NR}$ spectrum (400 $\left.\mathrm{MHz}\right)$ of $3 \boldsymbol{b}$ in $\mathrm{CDCl}_{3}$.

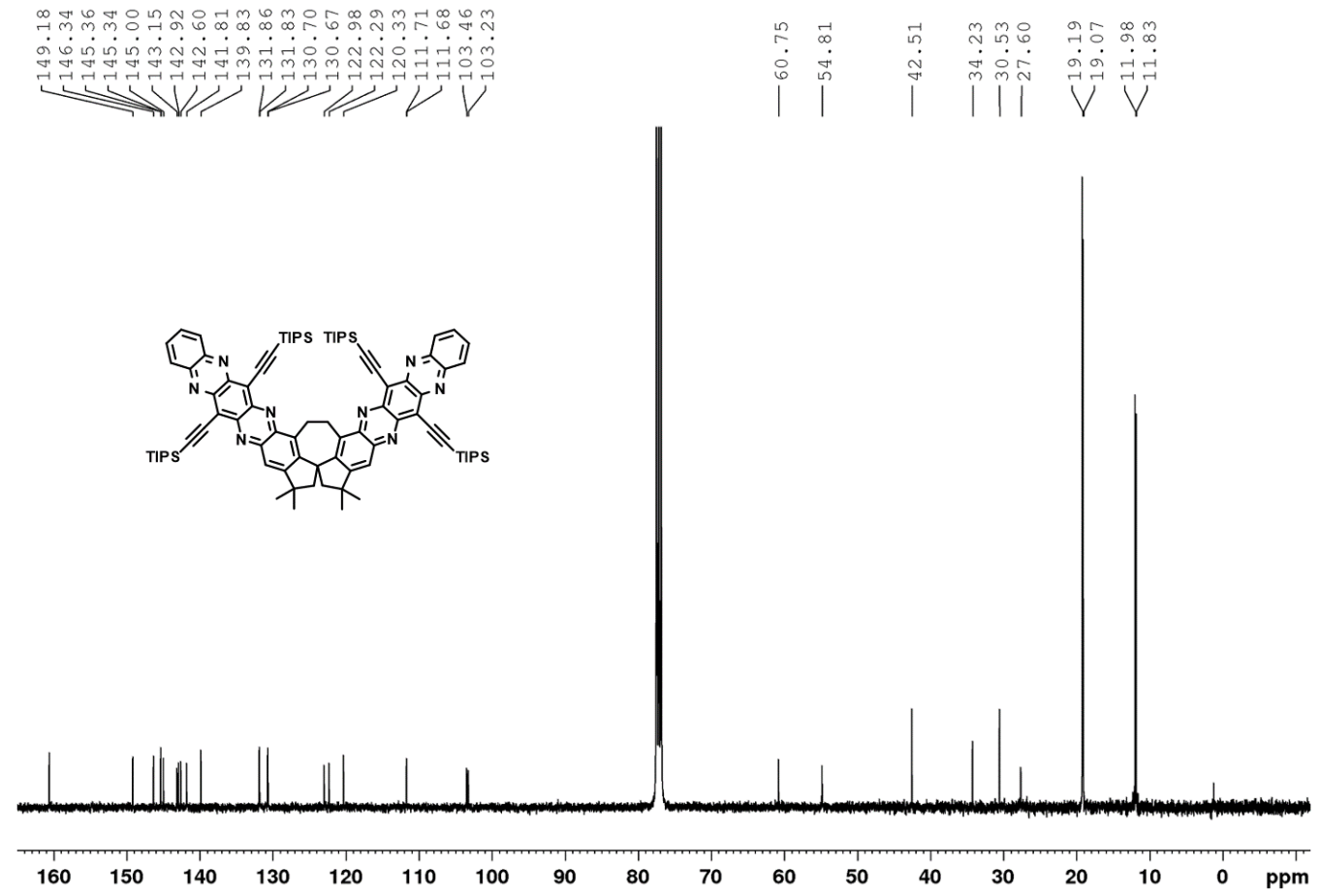

Figure S28: $\left.{ }^{13} C_{\{}^{\{l} \mathrm{H}\right\}$ NMR spectrum $(101 \mathrm{MHz})$ of $\mathbf{3} \boldsymbol{b}$ in $\mathrm{CDCl}_{3}$. 


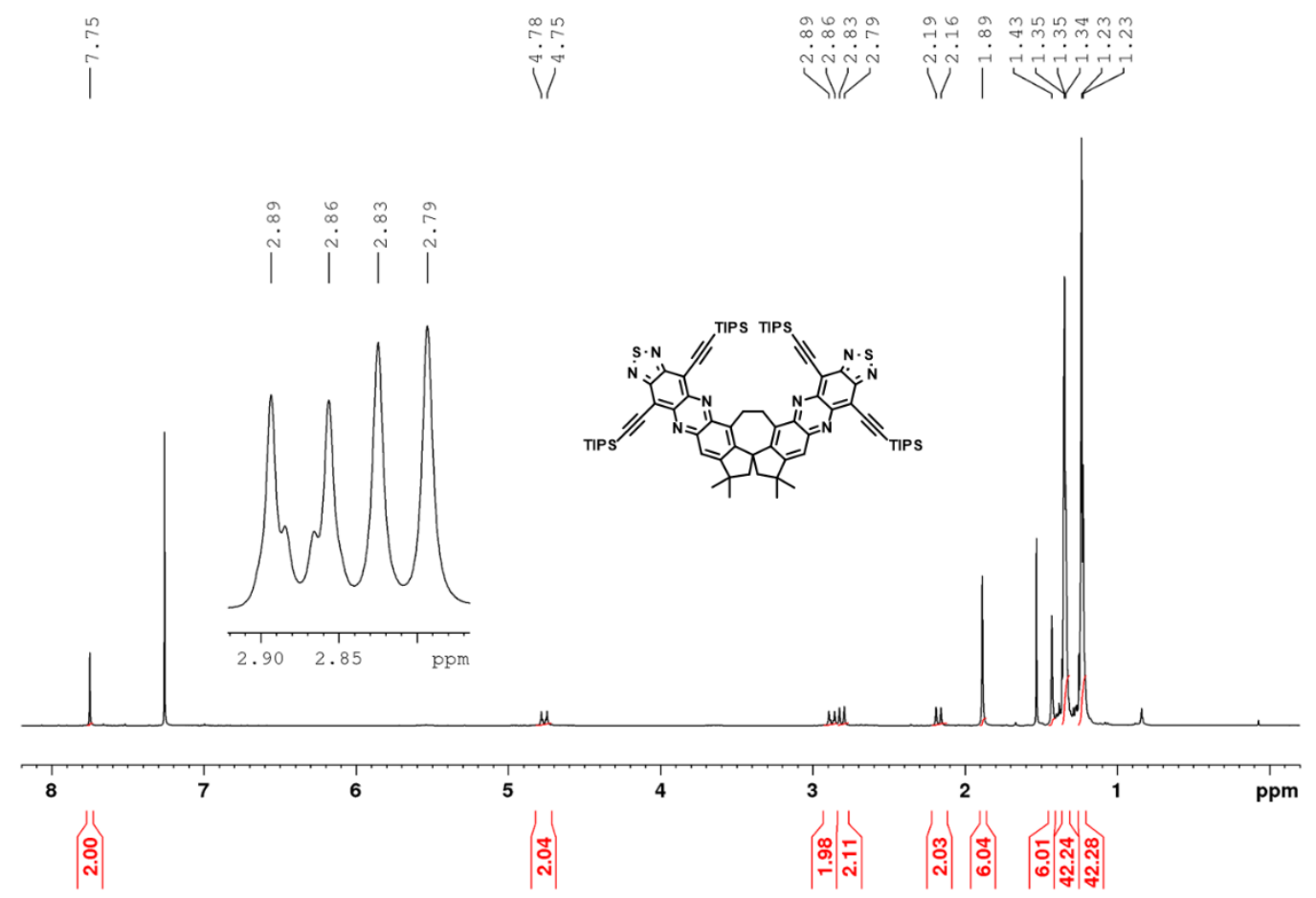

Figure S29: ${ }^{1} \mathrm{H} N \mathrm{NR}$ spectrum $(400 \mathrm{MHz})$ of $3 \mathrm{c}$ in $\mathrm{CDCl}_{3}$.

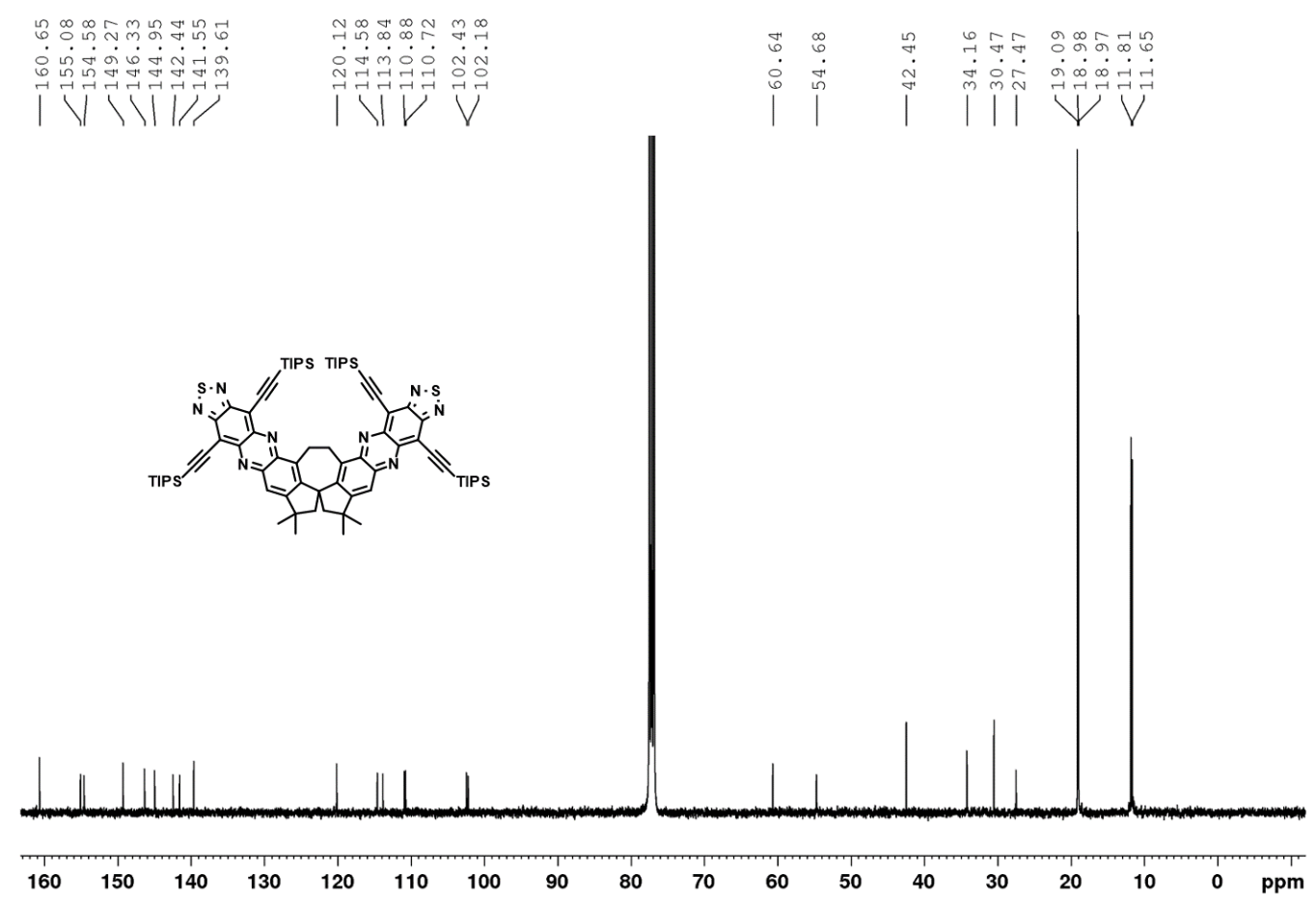

Figure S30: $\left.{ }^{13} C_{\{}^{\{1} H\right\}$ NMR spectrum (101 MHz) of $3 \boldsymbol{c}$ in $\mathrm{CDCl}_{3}$. 


\section{Cyclic Voltammetry}

Cyclic voltammetry experiments were carried out using PGSTAT101 Potentiostat Galvanostat with a platinum working electrode (Metrohm 6.120.4190, diameter $1 \mathrm{~mm}$ ), a platinum wire auxiliary electrode (Metrohm 3.109.0790), a silver wire reference electrode (Metrohm 6.1241.060), a $0.1 \mathrm{~mol} \mathrm{~L}^{-1} \mathrm{NBu} 4 \mathrm{PF}_{6}$ solution in degassed, dry dichloromethane, and ferrocene/ferrocenium as the reference redox system and internal standard $(-5.1 \mathrm{eV})$ at room temperature and $0.2 \mathrm{~V} \mathrm{~s}^{-1}$. To determine the reduction and oxidation potentials, the half-wave potentials were used. ${ }^{8}$
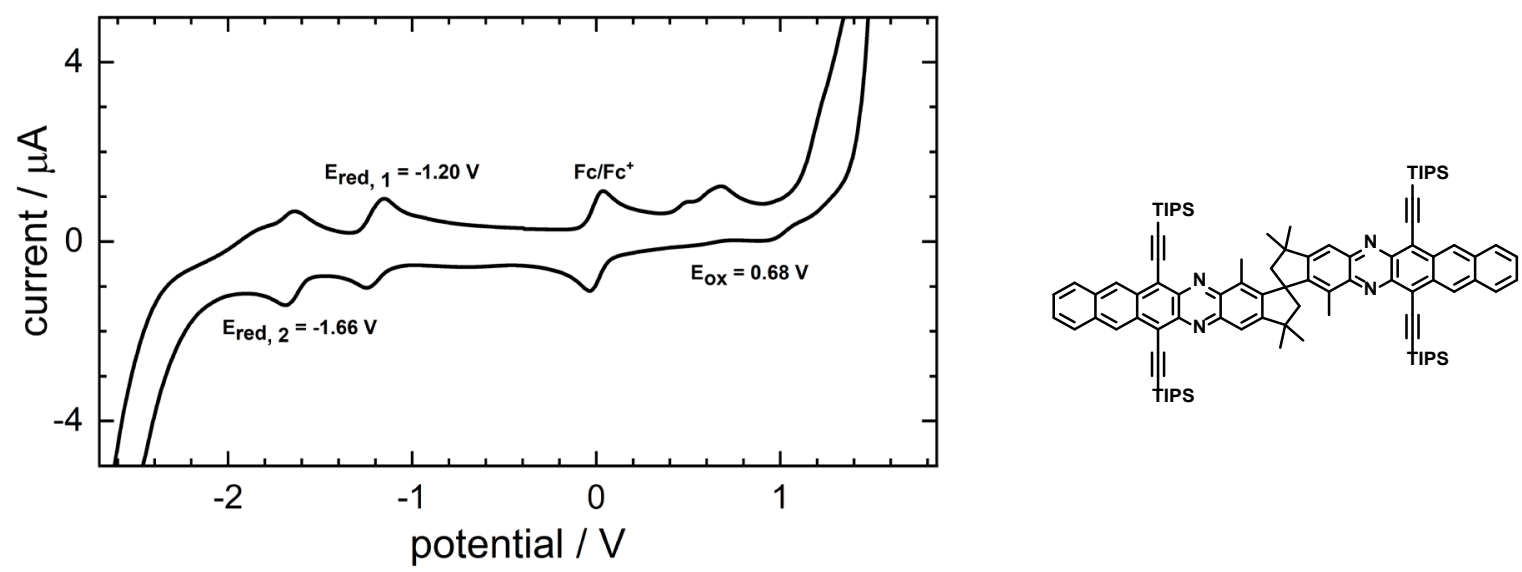

Figure S31: CV spectrum of 1a.
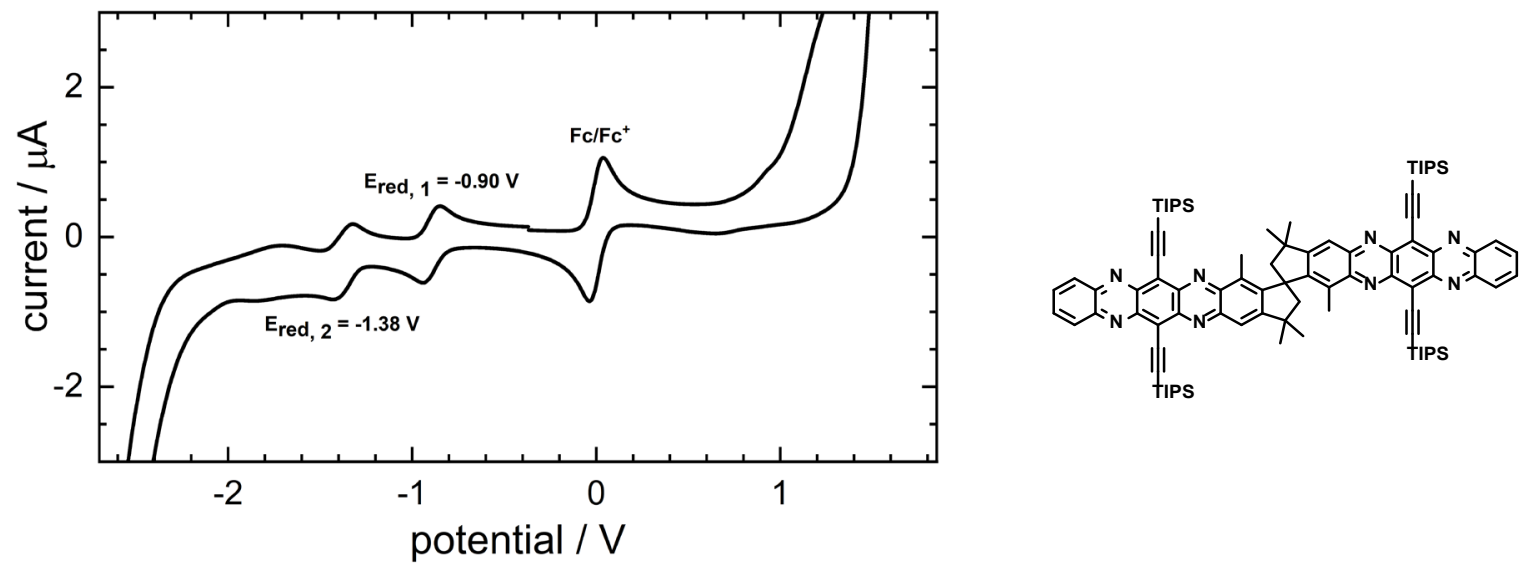

Figure S32: CV spectrum of $\mathbf{1 b}$. 

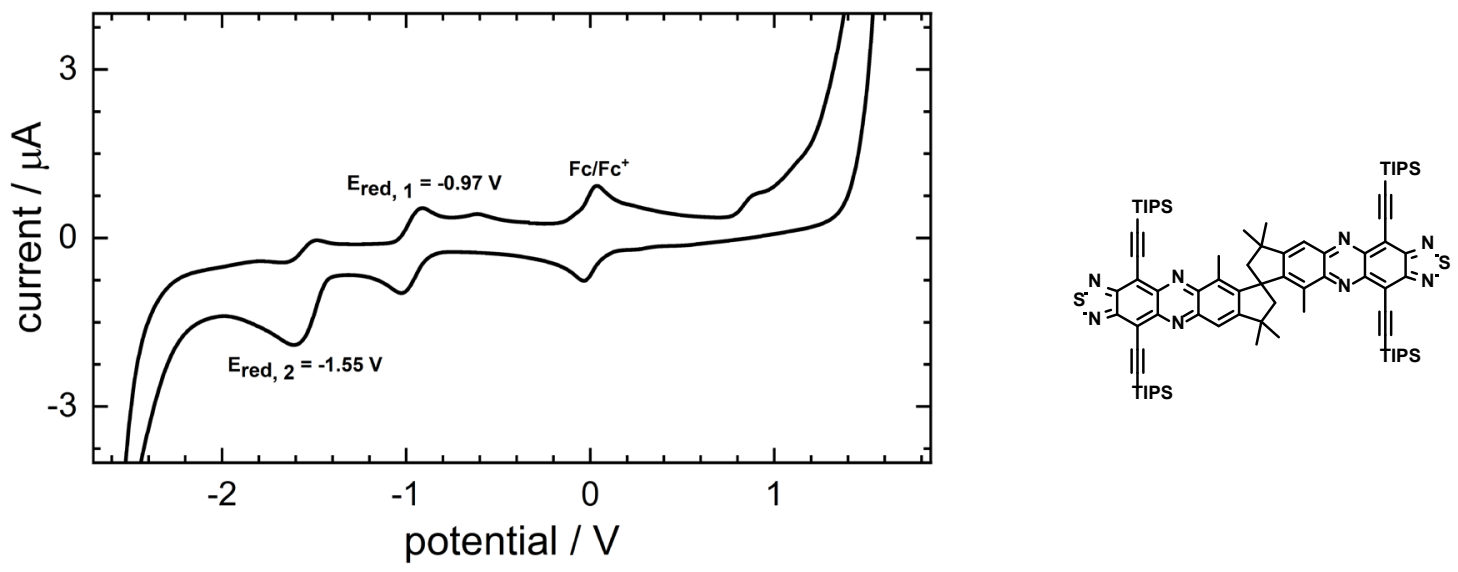

Figure S33: $C V$ spectrum of $1 c$.
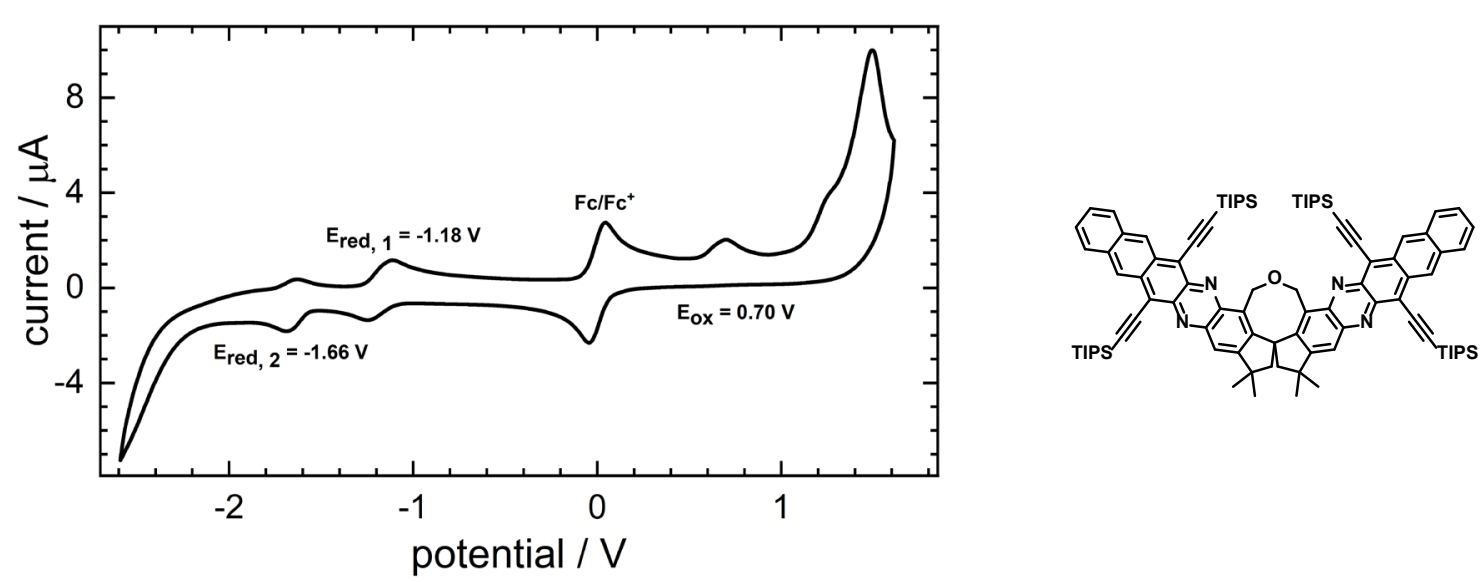

Figure S34: CV spectrum of $2 a$.
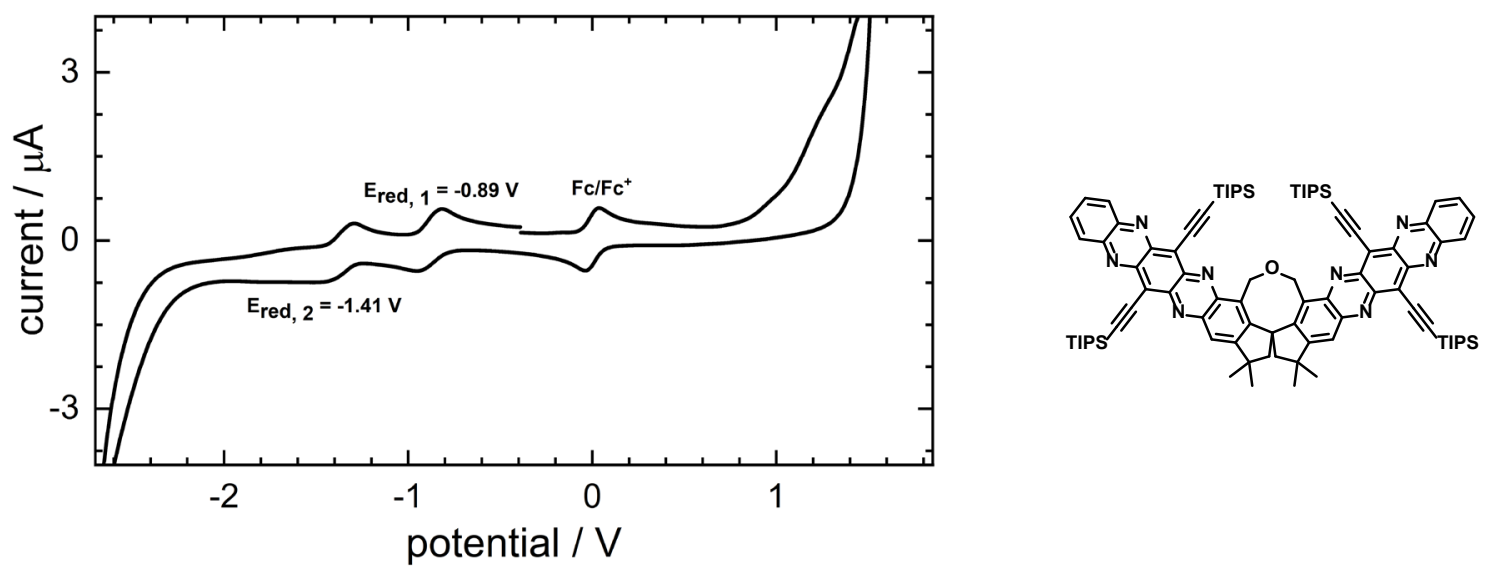

Figure S35: CV spectrum of $\mathbf{2 b}$. 

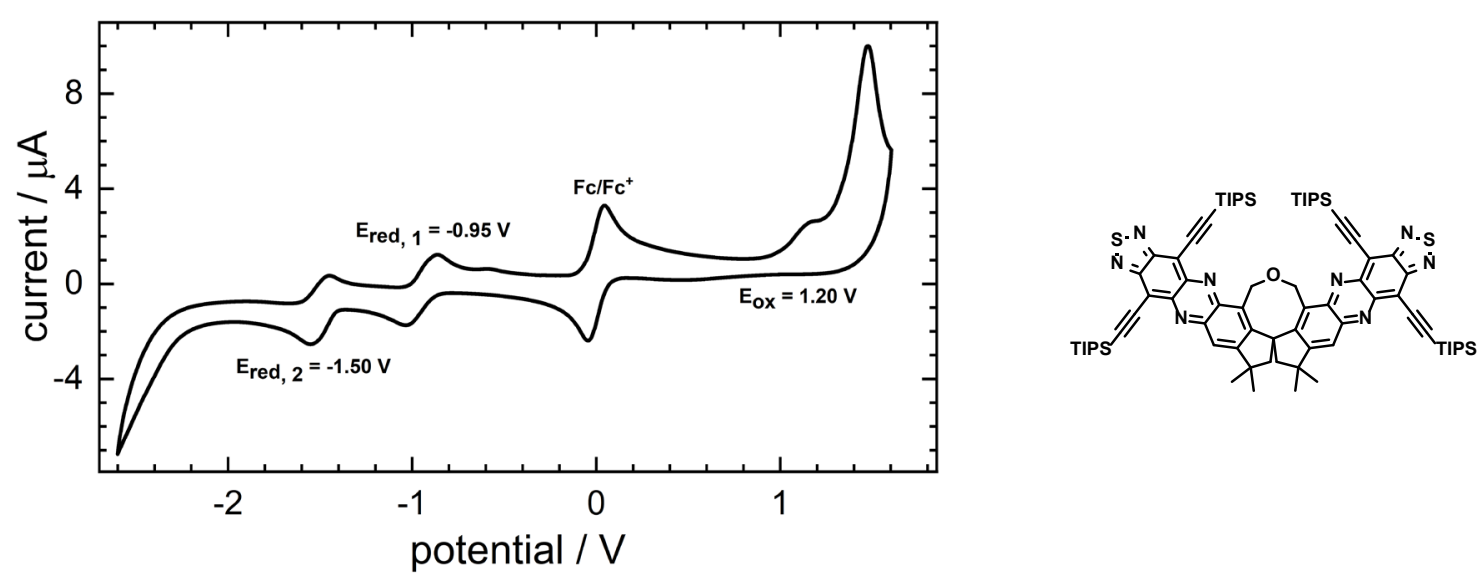

Figure S36: $C V$ spectrum of $2 c$.
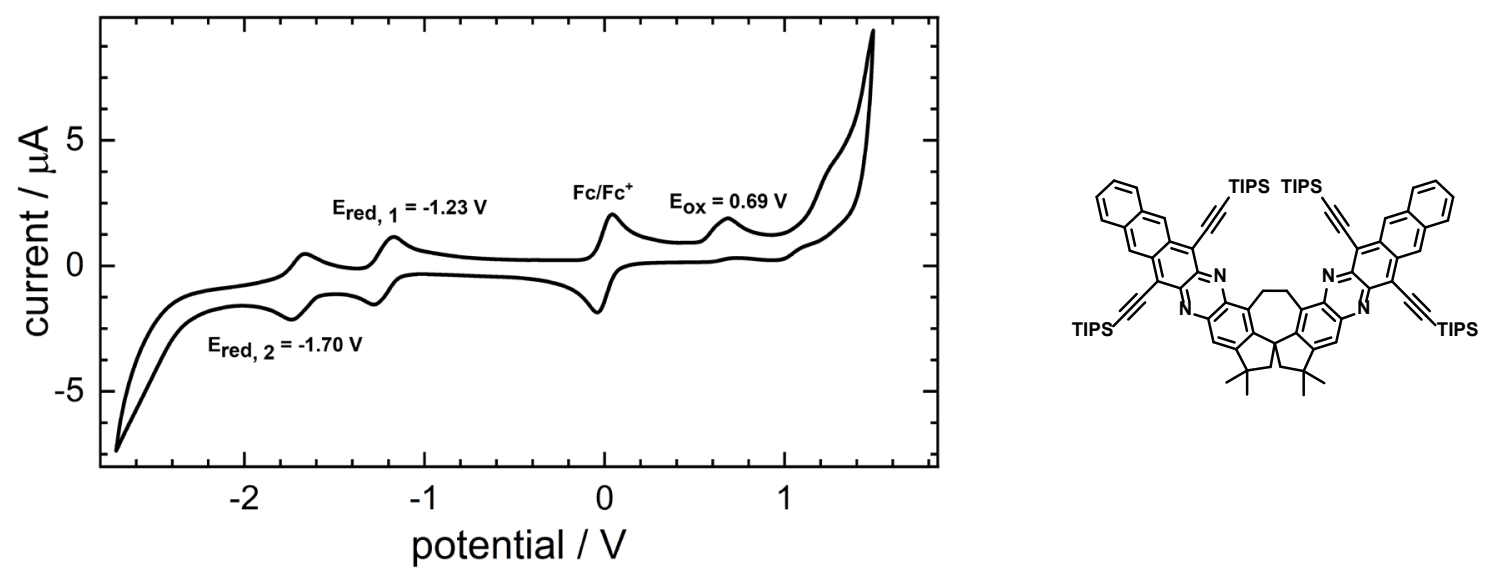

Figure S37: CV spectrum of $3 a$.
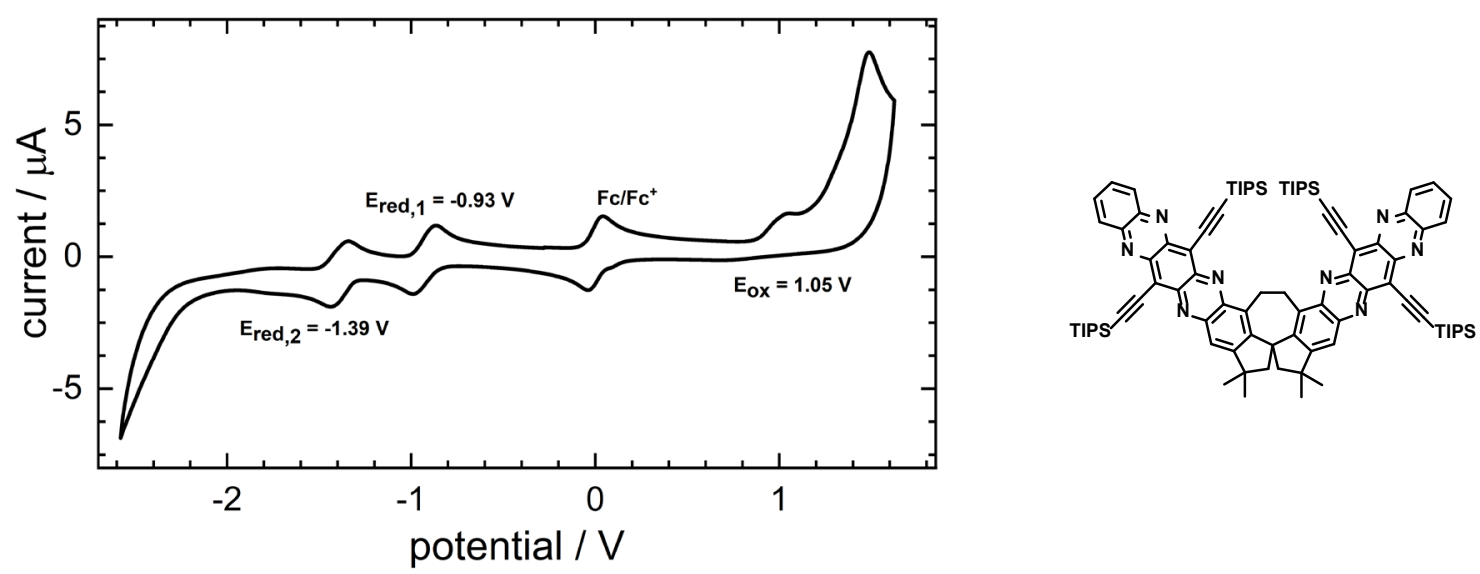

Figure S38: CV spectrum of $\mathbf{3 b}$. 

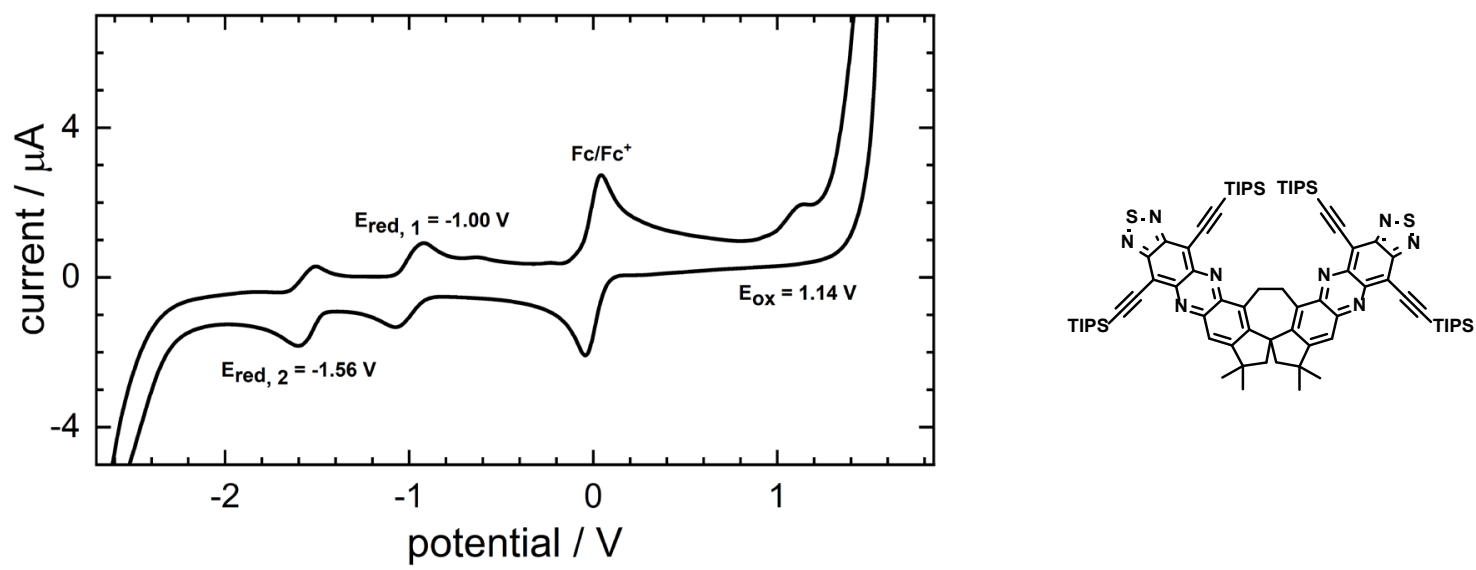

Figure S39: $C V$ spectrum of $3 c$.

Table S1: Experimental electrochemical properties of azaacene dimers $1 \boldsymbol{a}-3 \boldsymbol{c}$ in solution (dichloromethane).

\begin{tabular}{ccccc}
\hline Compd & $\begin{array}{c}E_{1 / 2}^{\text {red }} \\
{[\mathrm{V}]^{[\mathrm{a}]}}\end{array}$ & $\begin{array}{c}E A_{\mathrm{CV}} \\
{[\mathrm{eV}]^{[\mathrm{b}]}}\end{array}$ & $\begin{array}{c}E_{\text {gap, opt. }} \\
{[\mathrm{eV}]^{[\mathrm{c}]}}\end{array}$ & $\begin{array}{c}I P_{\text {opt. }} \\
{[\mathrm{eV}]^{[\mathrm{d}]}}\end{array}$ \\
\hline $\mathbf{1 a}$ & -1.20 & -3.90 & 1.71 & 5.61 \\
$\mathbf{2 a}$ & -1.18 & -3.92 & 1.69 & 5.61 \\
$\mathbf{3 a}$ & -1.23 & -3.87 & 1.72 & 5.59 \\
$\mathbf{1 b}$ & -0.90 & -4.20 & 1.73 & 5.93 \\
$\mathbf{2 b}$ & -0.89 & -4.21 & 1.74 & 5.95 \\
$\mathbf{3 b}$ & -0.93 & -4.17 & 1.74 & 5.91 \\
$\mathbf{1 c}$ & -0.97 & -4.13 & 1.85 & 5.98 \\
$\mathbf{2 c}$ & -0.95 & -4.15 & 1.85 & 6.00 \\
$\mathbf{3 c}$ & -1.00 & -4.10 & 1.85 & 5.95 \\
\hline
\end{tabular}

[a] First reduction potentials from cyclic voltammetry $(\mathrm{CV})$ in $\mathrm{DCM}$ at room temperature with $\mathrm{Bu}_{4} \mathrm{NPF}_{6}$ as electrolyte against $\mathrm{Fc} / \mathrm{Fc}^{+}$as internal standard $(-5.10 \mathrm{eV})$ at $0.2 \mathrm{Vs}^{-1} .{ }^{8}[\mathrm{~b}] E A_{\mathrm{CV}}=-\mathrm{e}\left(5.1 \mathrm{~V}+E_{1 / 2}^{r e d}\right)$. [c] $E_{\mathrm{gap}, \text { opt. }}$ calculated from $\lambda_{\text {onset, abs }}$ in $T H F$. [d] $I P_{\mathrm{opt} .}=E A_{\mathrm{CV}}-E_{\text {gap, opt. }}$ 


\section{Crystal Structures and Optimized Geometries}

Table S2: Crystal structure, crystal data and structure refinement of S6 (CCDC 2085737).

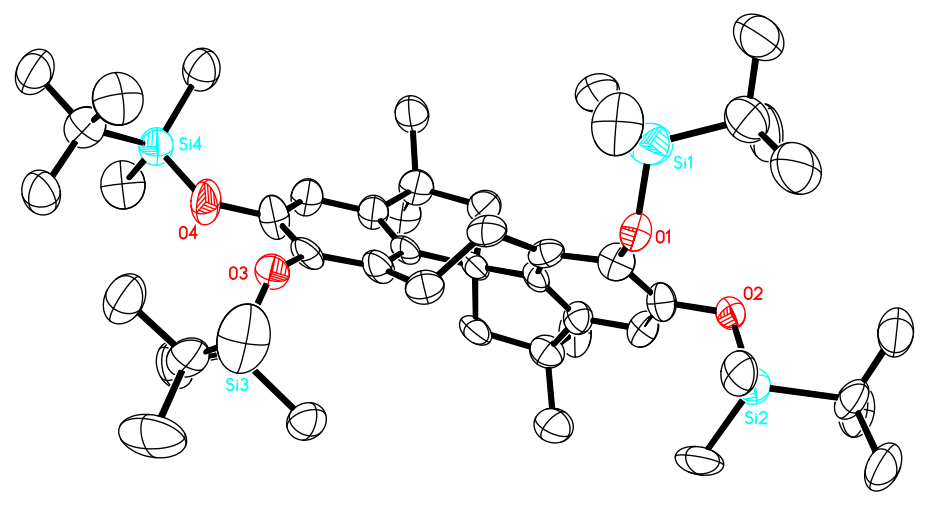

Identification code

Empirical formula

Formula weight

Temperature

Wavelength

Crystal system

Space group

Z

Unit cell dimensions

\section{Volume}

Density (calculated)

Absorption coefficient

Crystal shape

Crystal size

Crystal colour

Theta range for data collection

Index ranges

Reflections collected

Independent reflections

Observed reflections

Absorption correction

Max. and min. transmission

Refinement method

Data/restraints/parameters

Goodness-of-fit on $\mathrm{F}^{2}$

Final $\mathrm{R}$ indices (I>2sigma(I))

Largest diff. peak and hole
CCDC 2085737

$\mathrm{C}_{47} \mathrm{H}_{82} \mathrm{O}_{4} \mathrm{Si}_{4}$

823.48

200(2) K

$1.54178 \AA$

monoclinic

$\mathrm{P} 21 / \mathrm{n}$

4

$\mathrm{a}=10.3973(11) \AA \quad \mathrm{a}=90 \mathrm{deg}$.

$\mathrm{b}=34.436(5) \AA \quad \mathrm{b}=95.946(9) \mathrm{deg}$.

$\mathrm{c}=14.5834(16) \AA \quad \mathrm{g}=90 \mathrm{deg}$.

5193.3(10) $\AA^{3}$

$1.05 \mathrm{~g} / \mathrm{cm}^{3}$

$1.34 \mathrm{~mm}^{-1}$

plate

$0.098 \times 0.067 \times 0.012 \mathrm{~mm}^{3}$

colourless

3.3 to 42.5 deg.

$-8 \leq \mathrm{h} \leq 9,-29 \leq \mathrm{k} \leq 30,-12 \leq \mathrm{l} \leq 12$

15540

$3583(\mathrm{R}(\mathrm{int})=0.2178)$

$1485(\mathrm{I}>2 \sigma(\mathrm{I}))$

Semi-empirical from equivalents

1.46 and 0.60

Full-matrix least-squares on $\mathrm{F}^{2}$

3583 / 656 / 520

0.91

$\mathrm{R} 1=0.068, \mathrm{wR} 2=0.129$

0.44 and $-0.26 \mathrm{e}^{-3}$ 
Table S3: Crystal structure, crystal data and structure refinement of 5 (CCDC 2085738).

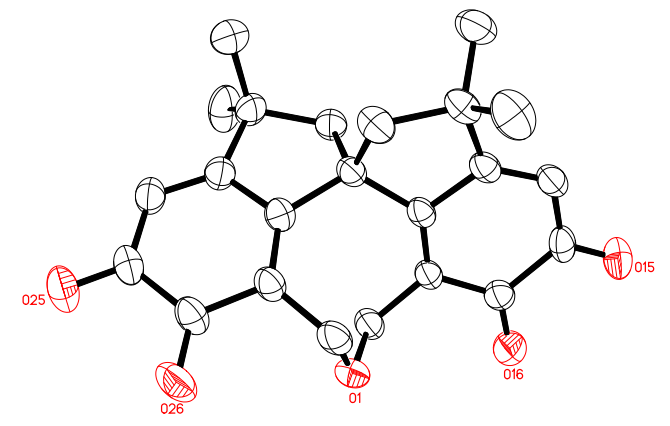

Identification code

Empirical formula

Formula weight

Temperature

Wavelength

Crystal system

Space group

Z

Unit cell dimensions

Volume

Density (calculated)

Absorption coefficient

Crystal shape

Crystal size

Crystal colour

Theta range for data collection

Index ranges

Reflections collected

Independent reflections

Observed reflections

Absorption correction

Max. and min. transmission

Refinement method

Data/restraints/parameters

Goodness-of-fit on $\mathrm{F}^{2}$

Final R indices (I>2sigma(I))

Largest diff. peak and hole
CCDC 2085738

$\mathrm{C}_{23.40} \mathrm{H}_{23.17} \mathrm{Cl}_{0.02} \mathrm{O}_{5.40}$

391.42

200(2) K

$1.54178 \AA$

monoclinic

$\mathrm{C} 2 / \mathrm{c}$

20

$\mathrm{a}=42.861(2) \AA \quad \mathrm{a}=90 \mathrm{deg}$.

$\mathrm{b}=9.1509(3) \AA \quad \mathrm{b}=96.835(4) \mathrm{deg}$.

$\mathrm{c}=25.3723(12) \AA \quad \mathrm{g}=90 \mathrm{deg}$.

9880.6(7) $\AA^{3}$

$1.32 \mathrm{~g} / \mathrm{cm}^{3}$

$0.78 \mathrm{~mm}^{-1}$

plank

$0.124 \times 0.113 \times 0.023 \mathrm{~mm}^{3}$

orange

2.1 to $66.6 \mathrm{deg}$.

$-38 \leq \mathrm{h} \leq 50,-10 \leq \mathrm{k} \leq 10,-25 \leq \mathrm{l} \leq 30$

40195

$8667(\mathrm{R}($ int $)=0.0561)$

$5420(\mathrm{I}>2 \sigma(\mathrm{I}))$

Semi-empirical from equivalents

1.24 and 0.72

Full-matrix least-squares on $\mathrm{F}^{2}$

8667 / 0 / 667

1.02

$\mathrm{R} 1=0.058, \mathrm{wR} 2=0.136$

0.88 and -0.68 e $\AA^{-3}$ 
Table S4: Crystal structure, crystal data and structure refinement of 6 (CCDC 2085739).

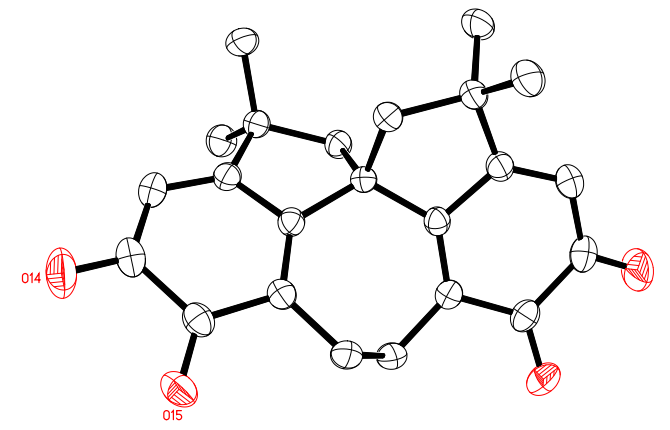

Identification code

Empirical formula

Formula weight

Temperature

Wavelength

Crystal system

Space group

$\mathrm{Z}$

Unit cell dimensions

Volume

Density (calculated)

Absorption coefficient

Crystal shape

Crystal size

Crystal colour

Theta range for data collection

Index ranges

Reflections collected

Independent reflections

Observed reflections

Absorption correction

Max. and min. transmission

Refinement method

Data/restraints/parameters

Goodness-of-fit on $\mathrm{F}^{2}$

Final $\mathrm{R}$ indices $(\mathrm{I}>2 \operatorname{sigma}(\mathrm{I}))$

Largest diff. peak and hole
CCDC 2085739

$\mathrm{C}_{23} \mathrm{H}_{22} \mathrm{O}_{4}$

362.40

200(2) K

$1.54178 \AA$

monoclinic

$\mathrm{C} 2 / \mathrm{c}$

4

$\mathrm{a}=19.4498(10) \AA \quad \mathrm{a}=90 \mathrm{deg}$.

$\mathrm{b}=9.0182(3) \AA \quad \mathrm{b}=127.754(3) \mathrm{deg}$.

$\mathrm{c}=13.1804(13) \AA \quad \mathrm{g}=90 \mathrm{deg}$.

$1827.9(2) \AA^{3}$

$1.32 \mathrm{~g} / \mathrm{cm}^{3}$

$0.72 \mathrm{~mm}^{-1}$

brick

$0.152 \times 0.091 \times 0.060 \mathrm{~mm}^{3}$

red

5.7 to $71.5 \mathrm{deg}$.

$-20 \leq \mathrm{h} \leq 23,-4 \leq \mathrm{k} \leq 10,-15 \leq \mathrm{l} \leq 14$

5759

$1741(\mathrm{R}($ int $)=0.0126)$

$1581(\mathrm{I}>2 \sigma(\mathrm{I}))$

Semi-empirical from equivalents

1.54 and 0.67

Full-matrix least-squares on $\mathrm{F}^{2}$

1741 / 0 / 125

1.08

$\mathrm{R} 1=0.040, \mathrm{wR} 2=0.104$

0.22 and $-0.22 \mathrm{e}^{-3}$ 
Table S5: Crystal structure, crystal data and structure refinement of 1a (CCDC 2085740).

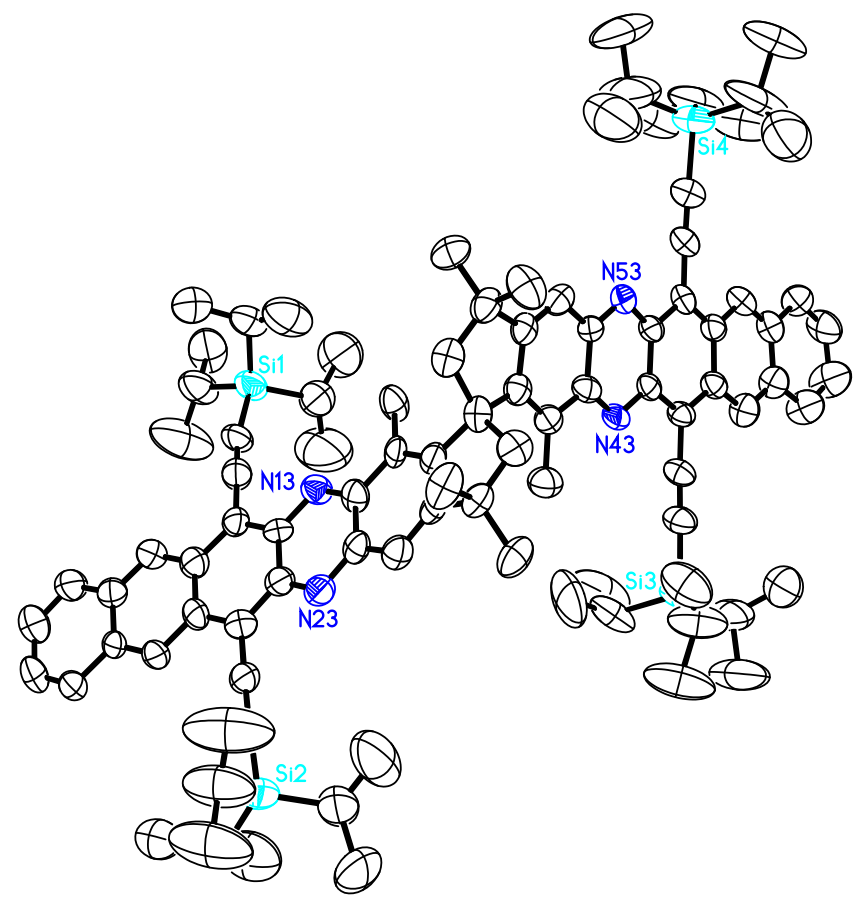

Identification code

Empirical formula

Formula weight

Temperature

Crystal system

Space group

Z

Unit cell dimensions

Volume

Density (calculated)

Absorption coefficient

Crystal shape

Crystal size

Crystal colour

Theta range for data collection

Index ranges

Reflections collected

Independent reflections

Observed reflections

Absorption correction

Max. and min. transmission

Refinement method

Data/restraints/parameters

Goodness-of-fit on $\mathrm{F}^{2}$

Final R indices ( $\mathrm{I}>2 \operatorname{sigma}(\mathrm{I})$ )

Absolute structure parameter

Largest diff. peak and hole
CCDC 2085740

$\mathrm{C}_{95.83} \mathrm{H}_{120.83} \mathrm{Cl}_{2.50} \mathrm{~N}_{4} \mathrm{Si}_{4}$

1529.78

200(2) K

monoclinic

$\mathrm{Cc}$

24

$\mathrm{a}=50.7427(18) \AA \quad \mathrm{a}=90 \mathrm{deg}$.

$\mathrm{b}=31.5332(8) \AA \quad \mathrm{b}=118.233(3) \mathrm{deg}$.

$\mathrm{c}=41.0801(15) \AA \quad \mathrm{g}=90 \mathrm{deg}$.

57911(4) $\AA^{3}$

$1.05 \mathrm{~g} / \mathrm{cm}^{3}$

$1.53 \mathrm{~mm}^{-1}$

plate

$0.170 \times 0.095 \times 0.022 \mathrm{~mm}^{3}$

green

3.0 to $50.4 \mathrm{deg}$.

$-50 \leq \mathrm{h} \leq 50,-31 \leq \mathrm{k} \leq 22,-39 \leq \mathrm{l} \leq 41$

124802

$49327(\mathrm{R}($ int $)=0.1907)$

$27191(\mathrm{I}>2 \sigma(\mathrm{I}))$

Semi-empirical from equivalents

1.50 and 0.64

Full-matrix least-squares on $\mathrm{F}^{2}$

49327 / 27287 / 5756

1.10

$\mathrm{R} 1=0.108, \mathrm{wR} 2=0.213$

$0.24(4)$

0.55 and $-0.30 \mathrm{e}^{-3}$ 
Table S6: Crystal structure, crystal data and structure refinement of 2c (CCDC 2085741).

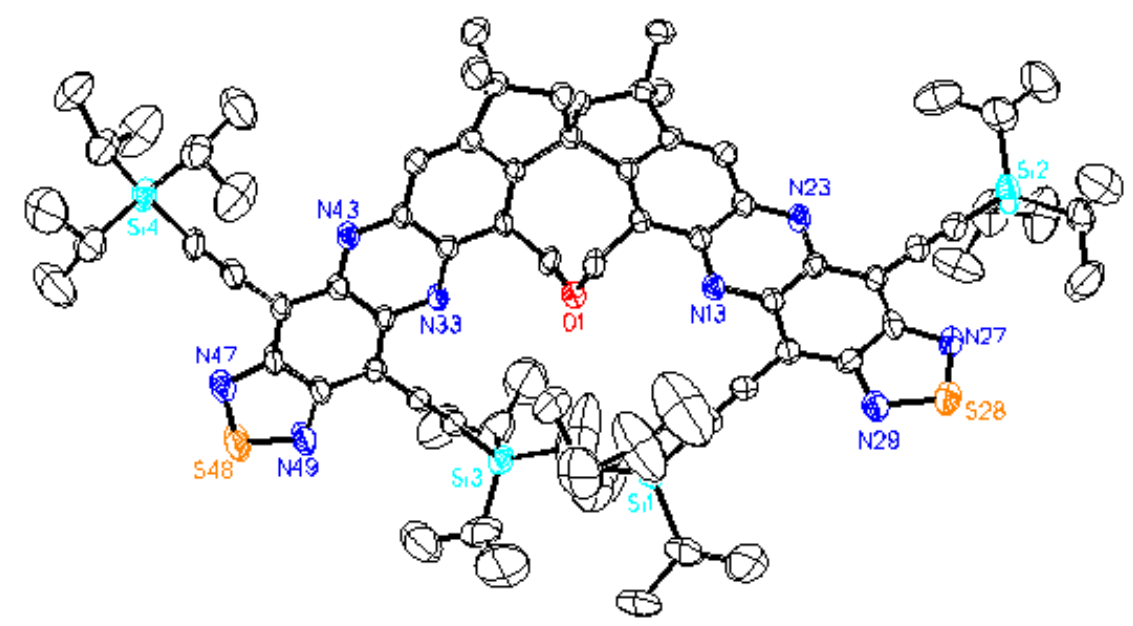

Identification code

Empirical formula

Formula weight

Temperature

Wavelength

Crystal system

Space group

Unit cell dimensions

Volume

Density (calculated)

Absorption coefficient

Crystal shape

Crystal size

Crystal colour

Theta range for data collection

Index ranges

Reflections collected

Independent reflections

Observed reflections

Absorption correction

Max. and min. transmission

Refinement method

Data/restraints/parameters

Goodness-of-fit on $\mathrm{F}^{2}$

Final $\mathrm{R}$ indices $(\mathrm{I}>2 \operatorname{sigma}(\mathrm{I}))$

Largest diff. peak and hole
CCDC 2085741

$\mathrm{C}_{79} \mathrm{H}_{106} \mathrm{~N}_{8} \mathrm{OS}_{2} \mathrm{Si}_{4}$

1360.19

$200(2) \mathrm{K}$

$1.54178 \AA$

triclinic

$\mathrm{P} \overline{1}$

$\mathrm{a}=96.310(5) \mathrm{deg}$.

$\mathrm{b}=15.3829(11) \AA \quad \mathrm{b}=108.019(5) \mathrm{deg}$.

$\mathrm{c}=20.0465(14) \AA \quad \mathrm{g}=89.662(6) \mathrm{deg}$.

4057.6(5) $\AA^{3}$

$1.11 \mathrm{~g} / \mathrm{cm}^{3}$

$1.51 \mathrm{~mm}^{-1}$

brick

$0.080 \times 0.070 \times 0.020 \mathrm{~mm}^{3}$

dark green

2.3 to $60.0 \mathrm{deg}$.

$-15 \leq \mathrm{h} \leq 15,-17 \leq \mathrm{k} \leq 17,-22 \leq \mathrm{l} \leq 22$

43002

$11578(\mathrm{R}(\mathrm{int})=0.0936)$

$6110(\mathrm{I}>2 \sigma(\mathrm{I}))$

Semi-empirical from equivalents

1.23 and 0.63

Full-matrix least-squares on $\mathrm{F}^{2}$

43002 / 471 / 876

0.87

$\mathrm{R} 1=0.070, \mathrm{wR} 2=0.153$

0.50 and $-0.50 \mathrm{e}^{-3}$ 
Table S7: Crystal structure, crystal data and structure refinement of 3c (CCDC 2085742).

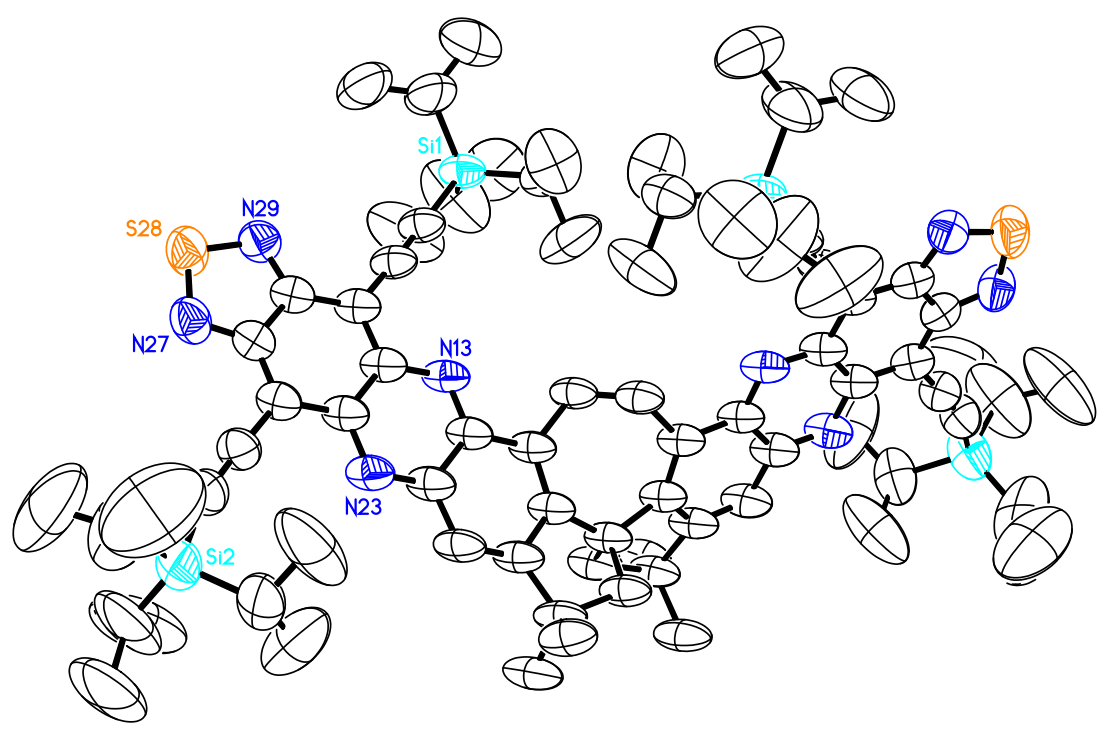

Identification code Empirical formula

Formula weight

Temperature

Wavelength

Crystal system

Space group

Z

Unit cell dimensions

Density (calculated)

Absorption coefficient

Crystal shape

Crystal size

Crystal colour

Theta range for data collection

Index ranges

Reflections collected

Independent reflections

Observed reflections

Absorption correction

Max. and min. transmission

Refinement method

Data/restraints/parameters

Goodness-of-fit on $\mathrm{F}^{2}$

Final $\mathrm{R}$ indices (I>2sigma $(\mathrm{I}))$

Largest diff. peak and hole
CCDC 2085742

$\mathrm{C}_{79} \mathrm{H}_{106} \mathrm{~N}_{8} \mathrm{~S}_{2} \mathrm{Si}_{4}$

1344.19

200(2) K

$1.54178 \AA$

monoclinic

$\mathrm{C} 2 / \mathrm{c}$

4

$\mathrm{a}=37.1165(18) \AA \quad \mathrm{a}=90 \mathrm{deg}$.

$\mathrm{b}=13.9744(4) \AA \quad \mathrm{b}=94.818(4) \mathrm{deg}$.

$\mathrm{c}=15.4894(6) \AA \quad \mathrm{g}=90 \mathrm{deg}$.

$1.12 \mathrm{~g} / \mathrm{cm}^{3}$

$1.52 \mathrm{~mm}^{-1}$

plate

$0.126 \times 0.111 \times 0.024 \mathrm{~mm}^{3}$

green

3.4 to $63.7 \mathrm{deg}$.

$-43 \leq \mathrm{h} \leq 42,-12 \leq \mathrm{k} \leq 16,-16 \leq \mathrm{l} \leq 18$

35963

$6577(\mathrm{R}(\mathrm{int})=0.0559)$

$3714(\mathrm{I}>2 \sigma(\mathrm{I}))$

Semi-empirical from equivalents

1.63 and 0.61

Full-matrix least-squares on $\mathrm{F}^{2}$

6577 / 1269 / 524

1.04

$\mathrm{R} 1=0.071, \mathrm{wR} 2=0.194$

0.31 and $-0.39 \mathrm{e}^{-3}$ 
a

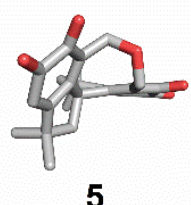

5 b

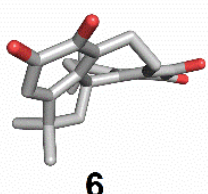

Figure S40: Crystal structures of quinones (a) 5 and (b) 6.

\section{Unlinked}

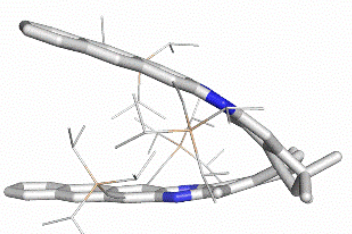

$1 a$

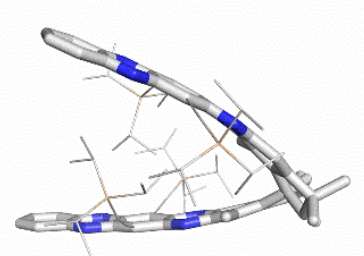

$1 b$

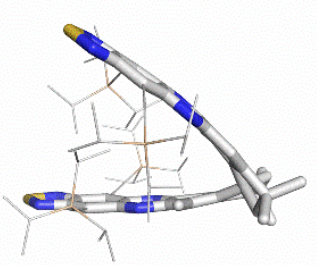

$1 c$
Ether-bridged

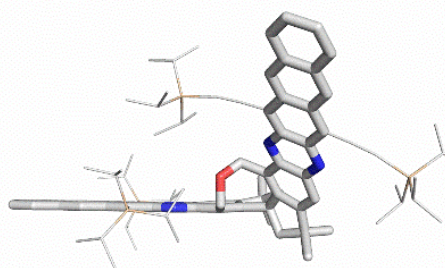

$2 a$

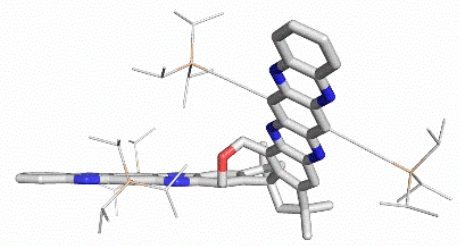

$2 b$

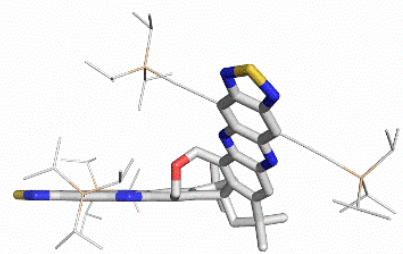

2c
Alkylene-bridged

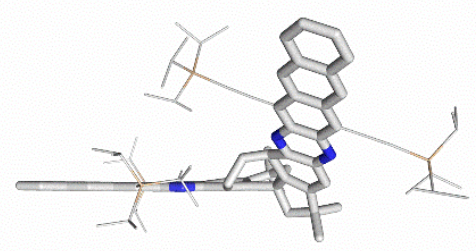

$3 a$

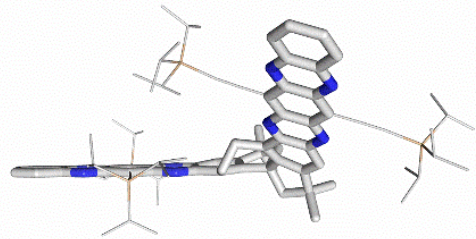

$3 b$

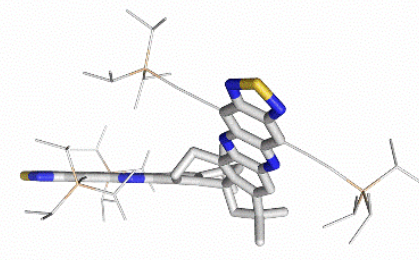

$3 c$

Figure S41: Optimized geometries obtained by quantum chemical calculations.. 


\section{Comparison of Experimental and Calculated Spectra}

5.1 Comparison of Steady-State Absorption Spectra and Calculated GroundState Transitions

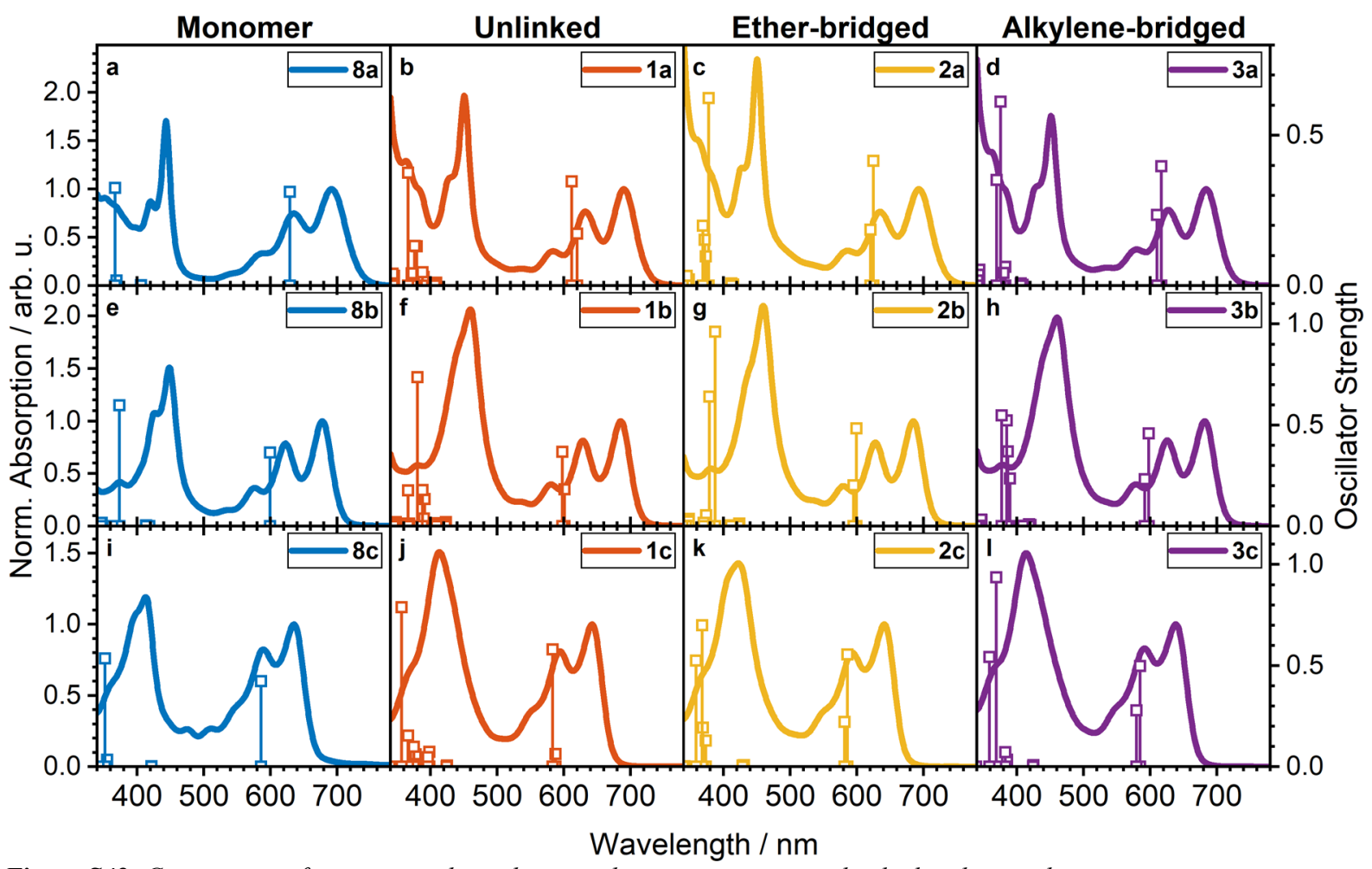

Figure S42: Comparison of experimental steady-state absorption spectra and calculated ground-state transitions. 
5.2 Comparison of SADS and Calculated Excited-State Transitions

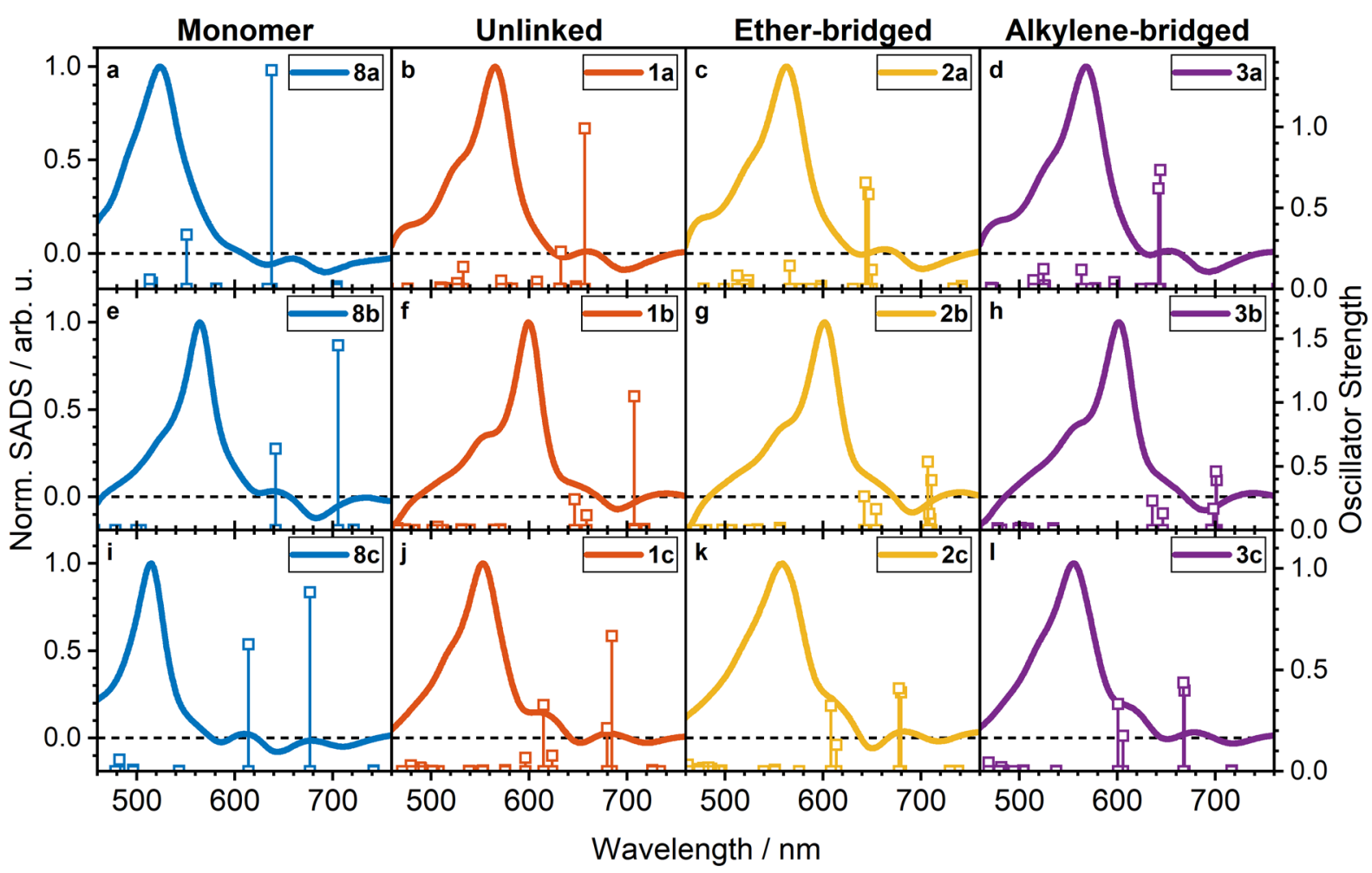

Figure S43: Comparison of $S_{1} S_{0} S A D S$ and calculated $S_{1} S_{0}$ ESA transitions.

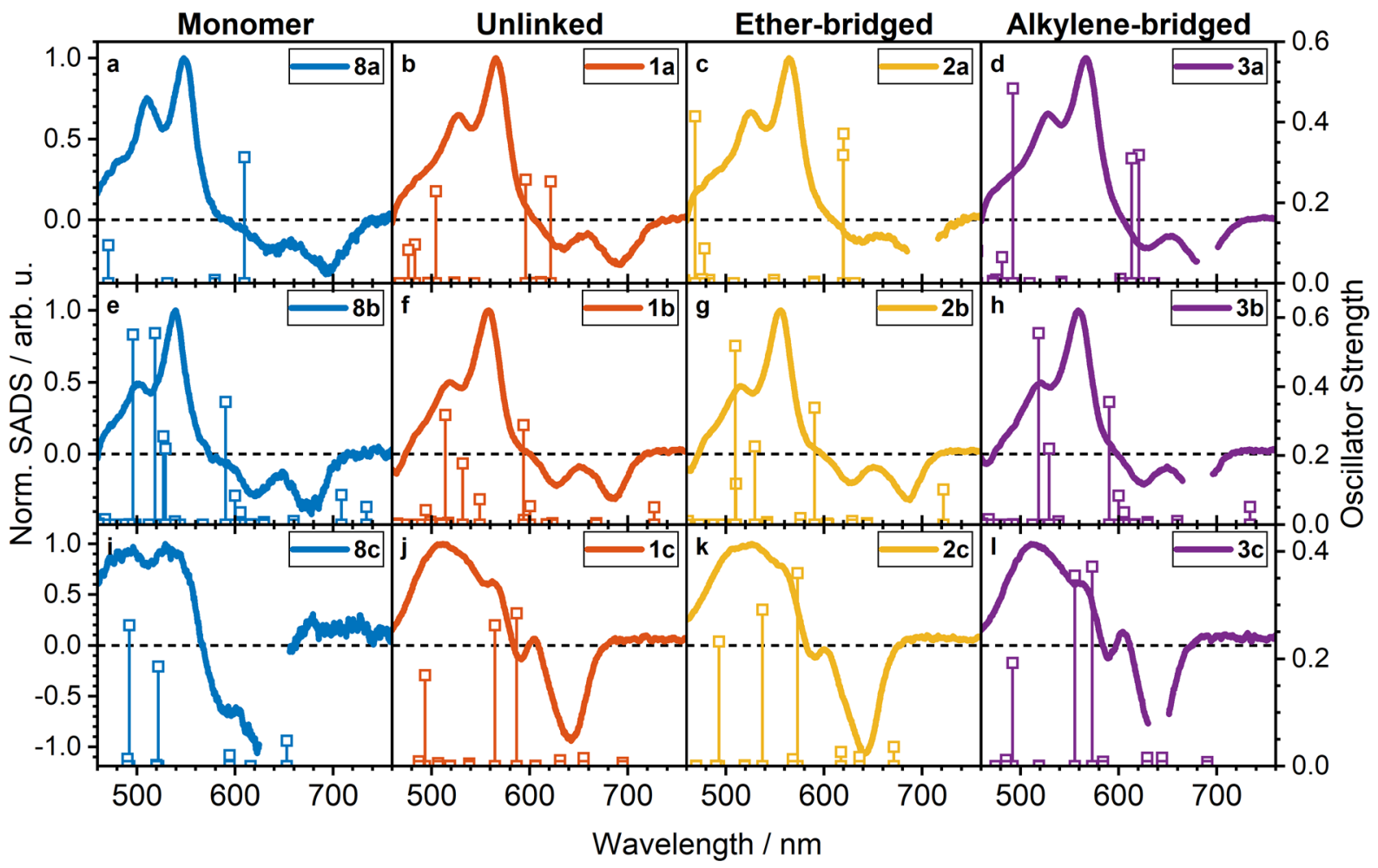

Figure S44: Comparison of $T_{1} S_{0} S A D S$ and calculated $T_{1} S_{0}$ ESA transitions. 


\section{Time-Resolved Spectroscopy}

\subsection{Monomers}
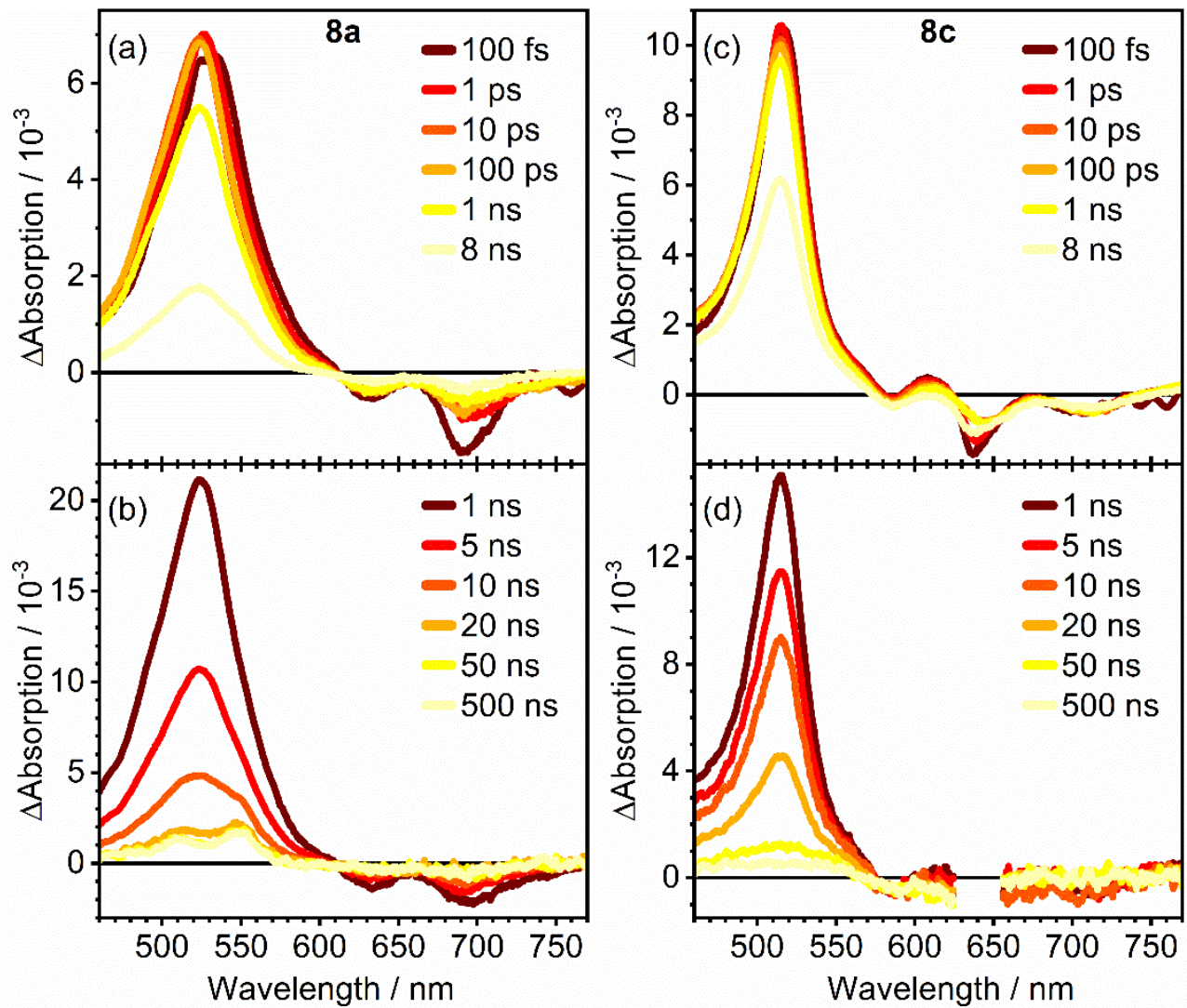

Figure S45: Transient absorption spectra at selected probe-delays. (a,b) Spectra of DAP-monomer 8a and (c,d) spectra of $P T D$-monomer $8 \mathrm{c}$ in the short- and long-time measurements, respectively.

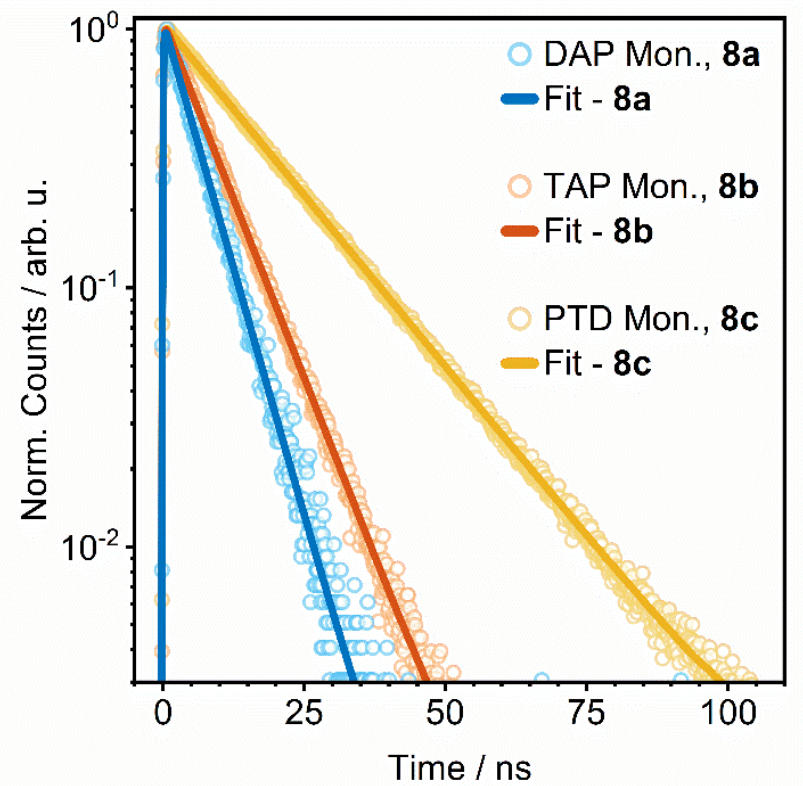

Figure S46: Time-resolved emission traces of monomers 8a-c. Experimental data points are displayed as circles and the fit curves are shown as solid lines. 
Table S8: Lifetimes obtained by global analysis of short- and long-time TA measurements $\left(\tau_{1}-\tau_{4}\right)$ and time-resolved emission $\left(\tau_{E m}\right)$ for monomers $\boldsymbol{8 a}, \boldsymbol{8 b}$ and $\boldsymbol{8 c}$.

\begin{tabular}{|c|c|c|c|c|}
\hline \multicolumn{2}{|c|}{ Lifetime } & 8a (DAP) & 8b (TAP) & 8c (PTD) \\
\hline \multirow{3}{*}{ Short-Time } & $\boldsymbol{\tau}_{\mathbf{1}} / \mathbf{p s}$ & $1.38 \pm 0.01$ & $1.47 \pm 0.01$ & $2.05 \pm 0.01$ \\
\cline { 2 - 5 } & $\boldsymbol{\tau}_{\mathbf{2}} / \mathbf{n s}$ & $5.3 \pm 0.1$ & $6.6 \pm 0.1$ & $10.1 \pm 0.1$ \\
\hline \multirow{3}{*}{ Long-Time } & $\boldsymbol{\tau}_{\mathbf{2}}^{\prime} / \mathbf{n s}$ & $5.5 \pm 0.1$ & $7.5 \pm 0.1$ & $15.6 \pm 0.1$ \\
\cline { 2 - 5 } & $\boldsymbol{\tau}_{\mathbf{3}} / \mathbf{n s}$ & $418 \pm 2$ & $400^{\mathrm{a}}$ & $250^{\mathrm{a}}$ \\
\cline { 2 - 5 } & $\boldsymbol{\tau}_{\mathbf{4}} / \mathbf{n s}$ & $950 \pm 10$ & $1010 \pm 20$ & $1130 \pm 20$ \\
\cline { 2 - 5 } & $\boldsymbol{\tau}_{\boldsymbol{E}} / \mathbf{n s}$ & $5.84 \pm 0.01$ & $7.97 \pm 0.01$ & $16.47 \pm 0.01$ \\
\hline
\end{tabular}

${ }^{a}$ Time constants were fixed during the fitting.
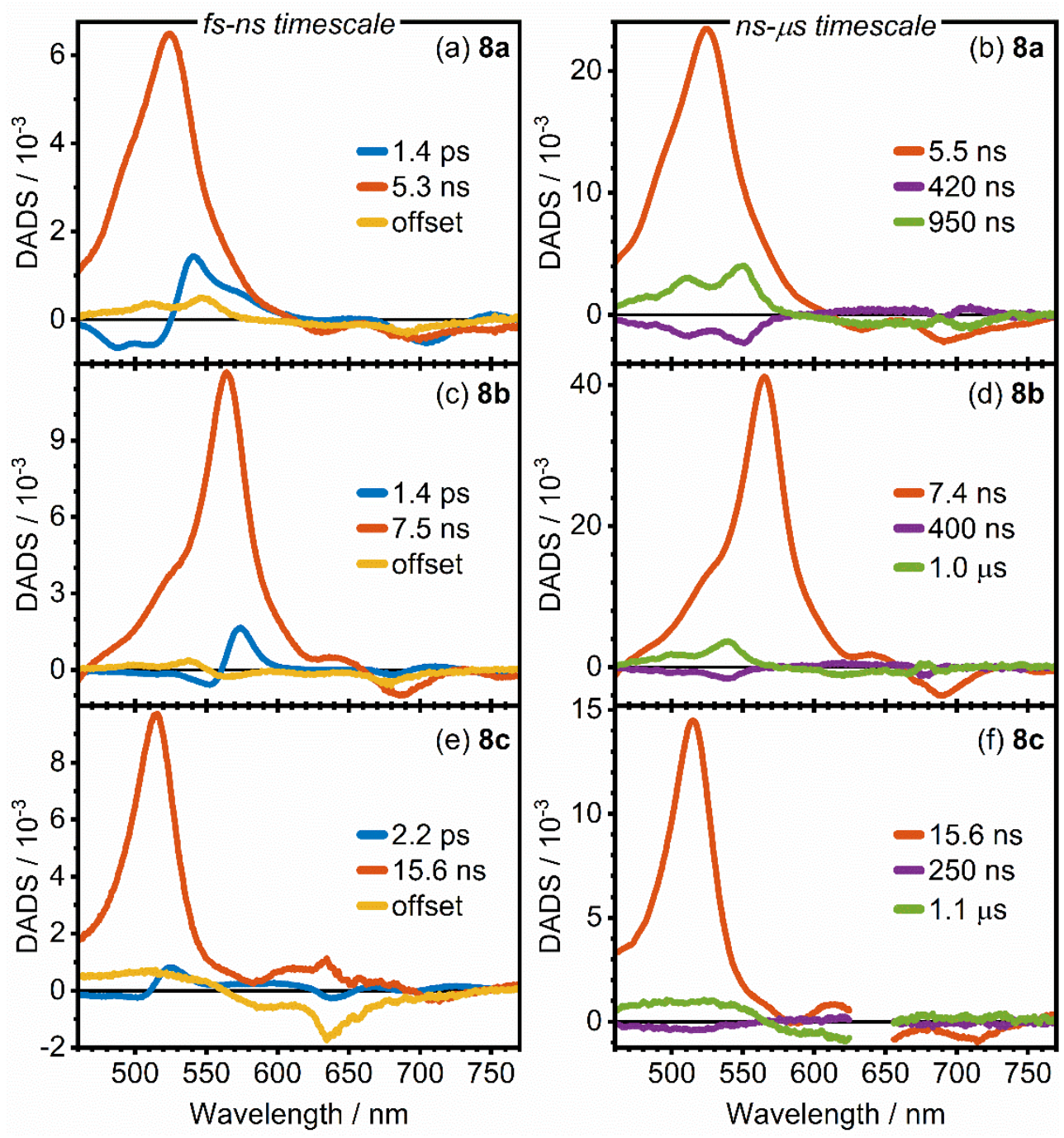

Figure S47: Decay-associated difference spectra (DADS) obtained by global analysis of TA measurements of monomers 8a$c$. The left column $(a, c, e)$ shows the DADS of the short-time measurements whereas the right column $(b, d, f)$ shows the DADS of the long-time measurements for the DAP $(a, b), \operatorname{TAP}(c, d)$ and PTD $(e, f)$ monomers. The corresponding time constants (c.f. Table S8) are given in the plot. 

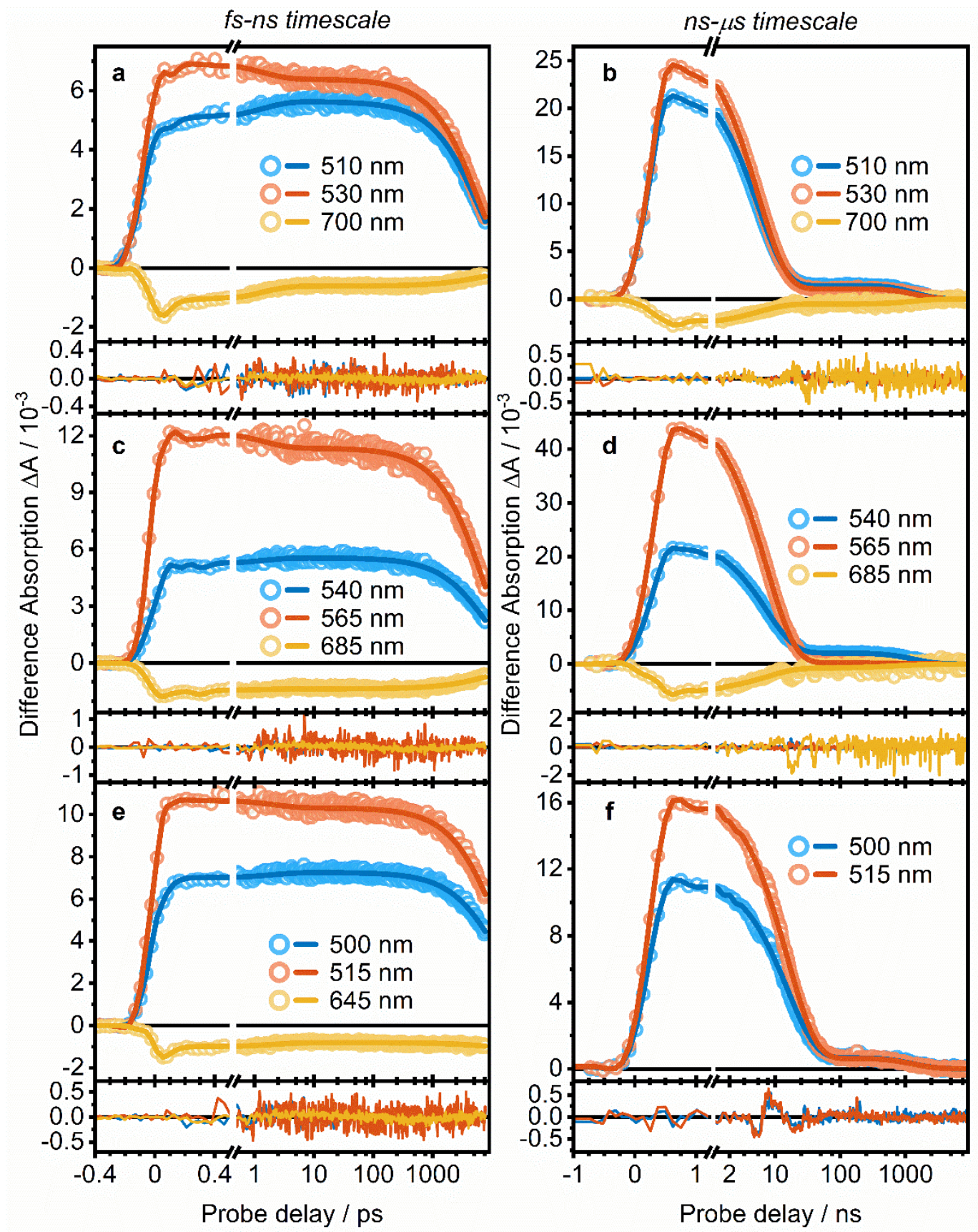

Figure S48: Fit of TA measurements of monomers 8a-c. Kinetic traces at selected wavelengths and fit curves are shown in (a) and (b) for $8 \boldsymbol{a}$, (c) and (d) for $\mathbf{8 b}$ and (e) and (f) for $\mathbf{8 c}$ for the $f s-n s-$ and $n s-\mu$ s time scale experiments, respectively. Residuals after subtraction of the fit are shown below the respective fit curves. Experimental data is shown as hollow circles, fit curves and residuals are shown as lines with the color coding for each selected wavelength shown in the legend. Notice the break after $0.5 \mathrm{ps}$ and $1.2 \mathrm{~ns}$ for the short-and long-timescale experiments, respectively, after which the probe delay is shown in a logarithmic scale. 

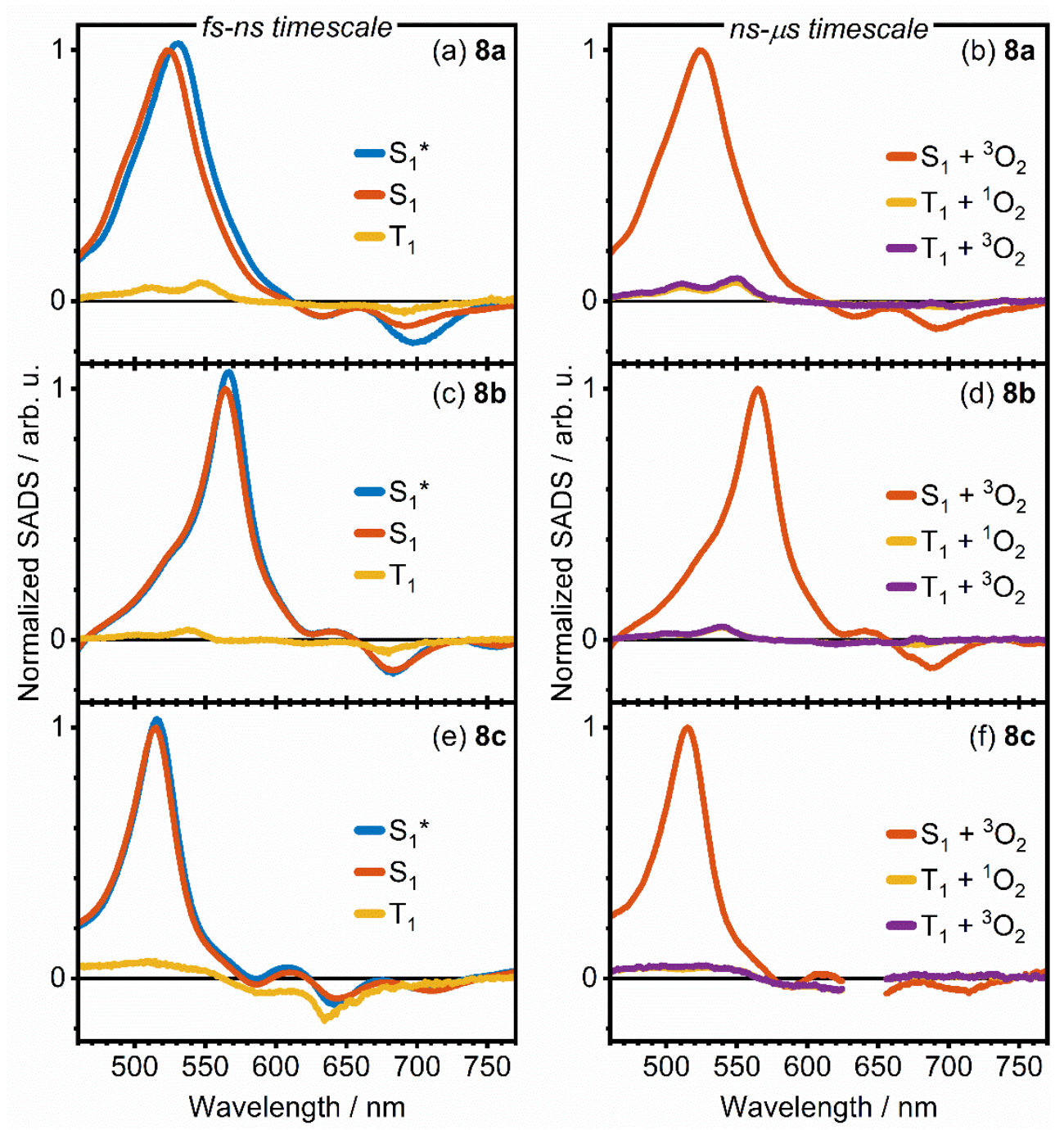

Figure S49: Species-associated difference spectra (SADS) obtained after applying a sequential kinetic model for the TA datasets in the short-time ( $a, c, e)$ and long-time measurements $(b, d, f)$ for the monomers $\boldsymbol{8} \boldsymbol{a}(a-b), \boldsymbol{8} \boldsymbol{b}(c-d)$ and $\boldsymbol{8 c}(e-f)$. See the Text for more information. The involved species shown in the legend are labelled in accordance with an oxygen-catalyzed sequential singlet fission model. ${ }^{9}$ 


\subsection{Dimers}

\subsubsection{Transient Absorption Spectra}
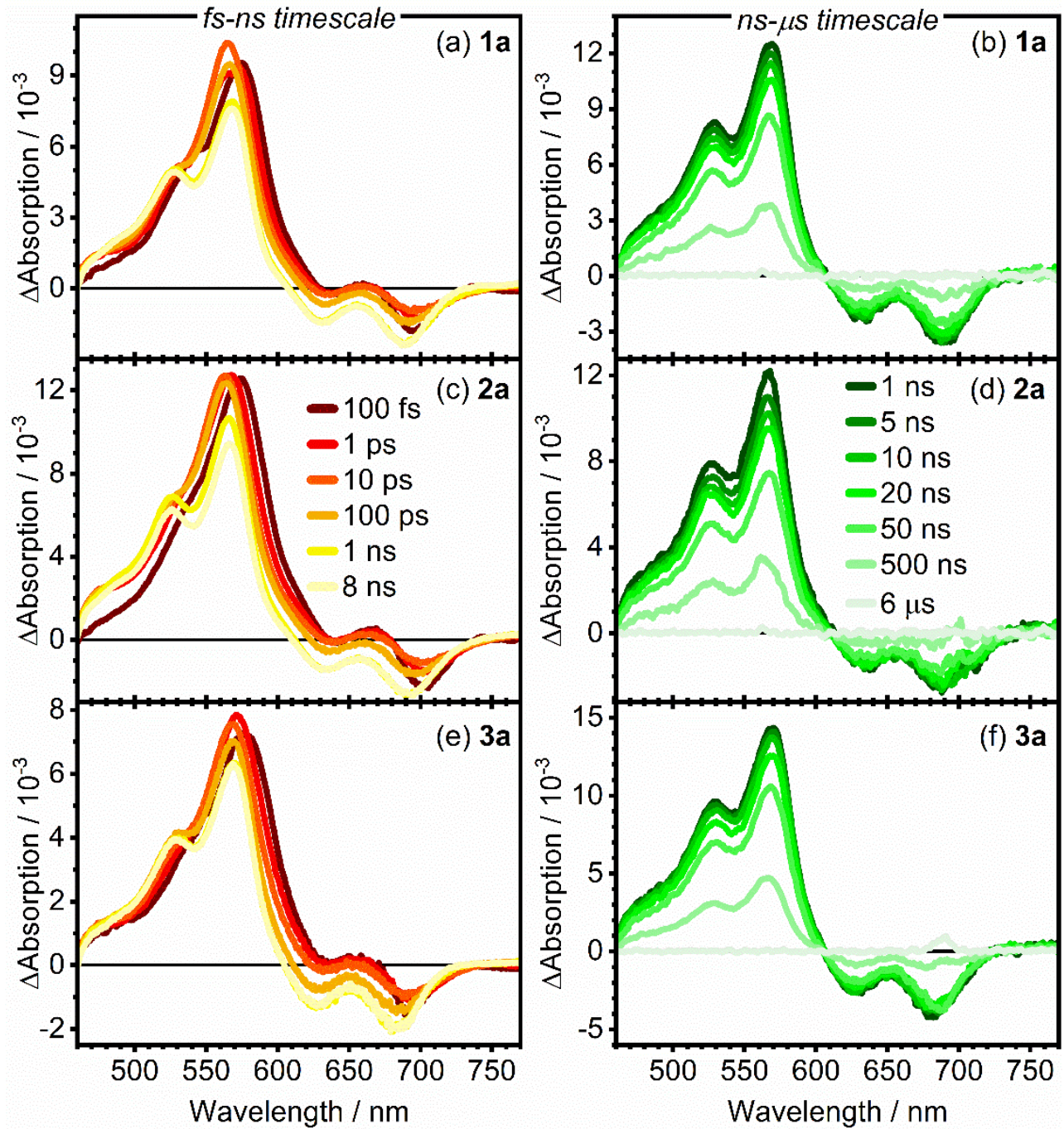

Figure S50: Transient absorption spectra of DAP-Dimers 1-3a at selected probe-delays. The short-time measurements are shown on the left $(a, c, e)$ and the long-time measurements on the right $(b, d, f)$ for $(a, b)$ unlinked 1a, (c,d) ether-bridged $2 a$ and $(e, f)$ alkylene-bridged $3 a$ DAP dimers. 

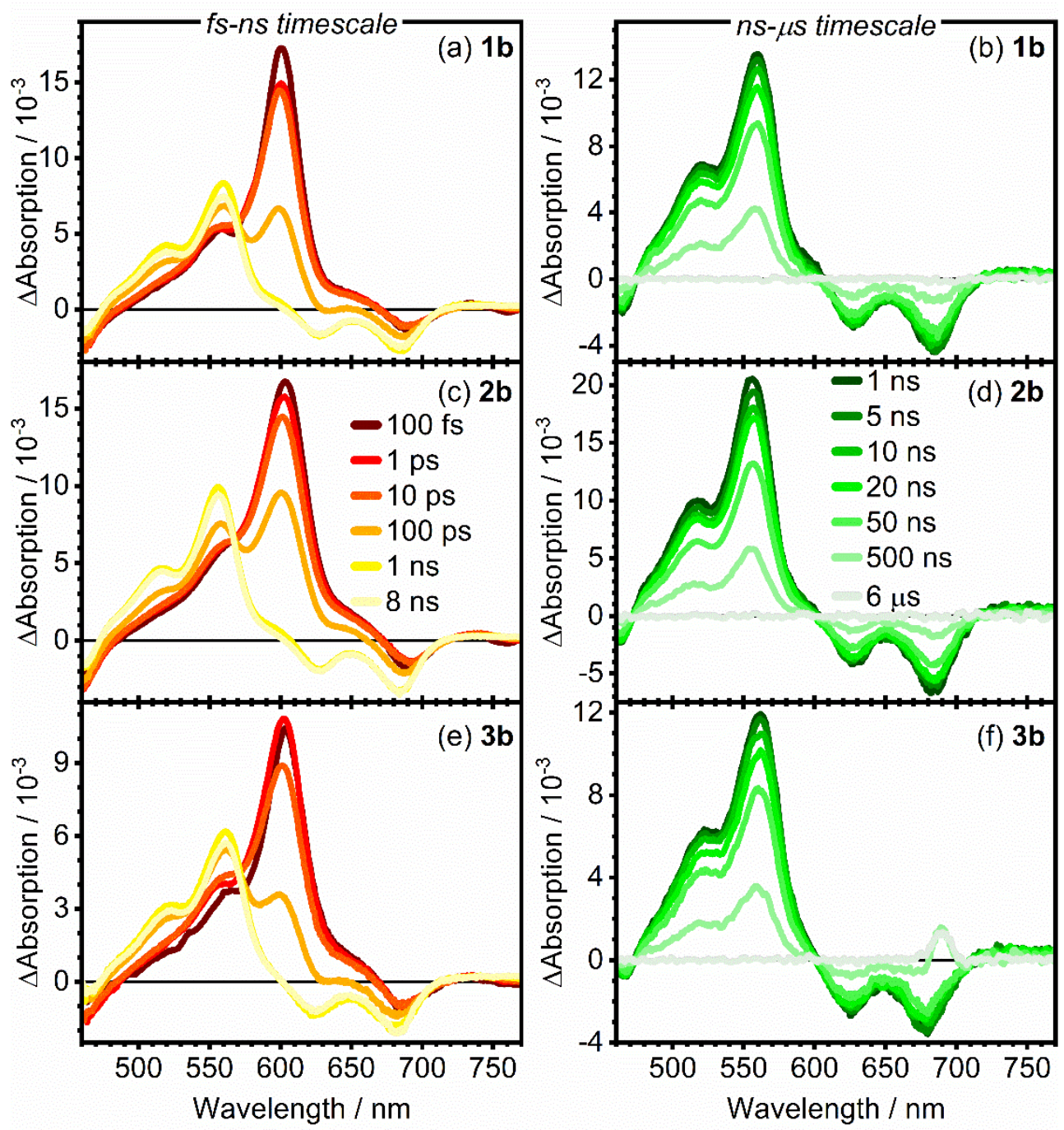

Figure S51: Transient absorption spectra of TAP-Dimers 1-3b at selected probe-delays. The short-time measurements are shown on the left $(a, c, e)$ and the long-time measurements on the right $(b, d, f)$ for $(a, b)$ unlinked $\mathbf{1 b},(c, d)$ ether-bridged $\mathbf{2 b}$ and (e,f) alkylene-bridged $\mathbf{3 b}$ TAP dimers. 

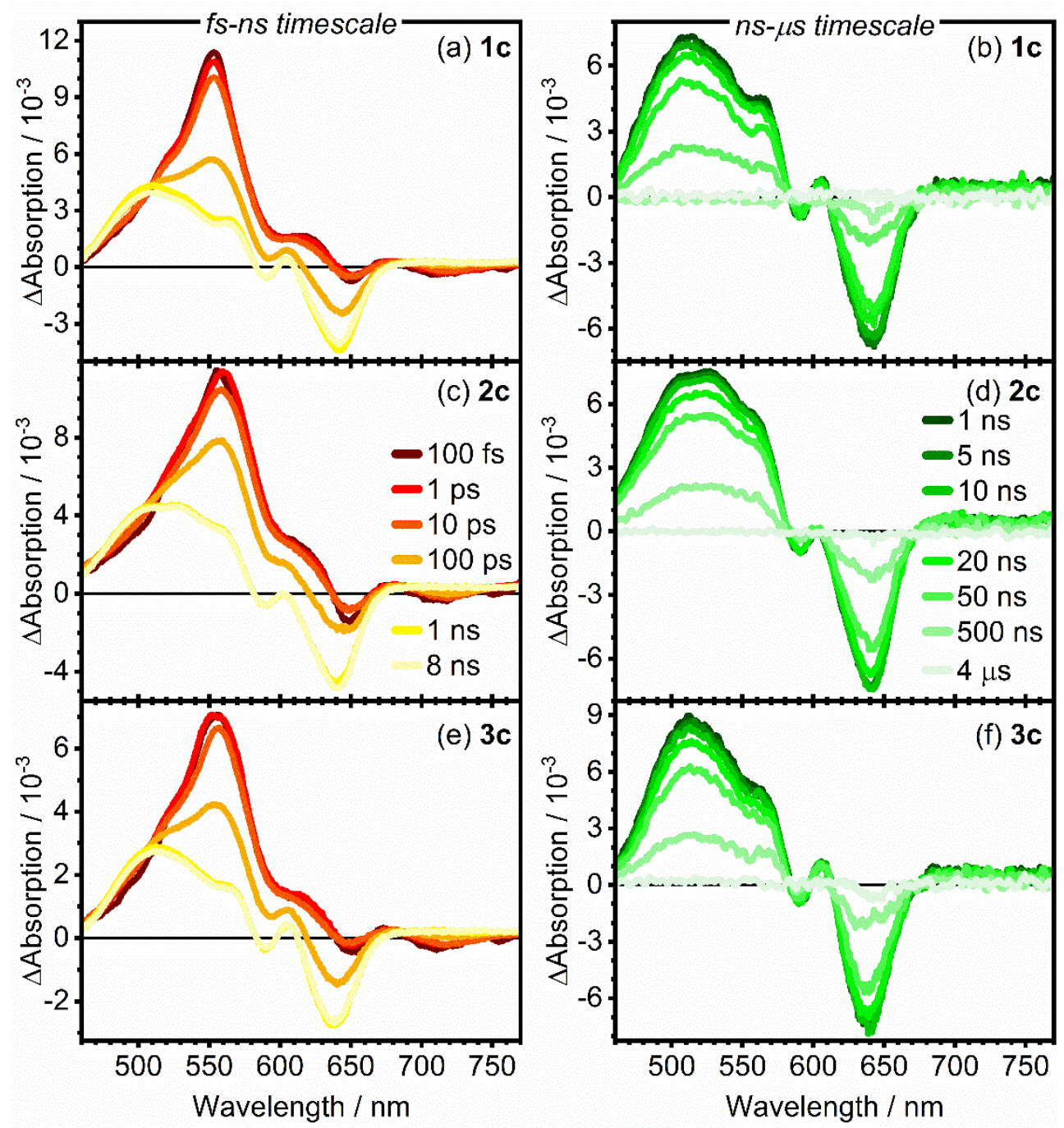

Figure S52: Transient absorption spectra of PTD-Dimers 1-3c at selected probe-delays. The short-time measurements are shown on the left $(a, c, e)$ and the long-time measurements on the right $(b, d, f)$ for $(a, b)$ unlinked $1 c$, (c,d) ether-bridged $2 c$ and (e,f) alkylene-bridged $3 c$ PTD dimers. 


\subsubsection{Global Analysis}

Table S2 summarizes the time constants obtained from global multiexponential fits. Long-time measurements were fitted with a three-exponential decay. Short-time measurements were fitted with three-exponential decay and an offset. As $\tau_{3}$ was typically on a similar time-scale compared to the highest possible probe delay in the short-time measurements ( $8 \mathrm{~ns})$, it was fixed to the value obtained from long-time measurements as typically being on for the global analysis of the latter. The only exception is $\mathbf{3 a}$, for which $\tau_{3}$ was obtained from short-time measurements and fixed in the global analysis of the long-time measurement. Using the branching ratios $\alpha$ displayed in table 4 in the main text, $\tau_{3 \mathrm{a}}$ and $\tau_{3 \mathrm{~b}}$ were calculated as $\tau_{3}^{a}=\left((1-\alpha) \cdot \frac{1}{\tau_{3}}\right)^{-1}$ and $\tau_{3}^{b}=\left(\alpha \cdot \frac{1}{\tau_{3}}\right)^{-1}$, respectively.

Table S9: Time constants obtained from global analysis of TA measurements $\left(\tau_{1}-\tau_{5}\right)$ and derived constants $\tau_{3}^{a}$ and $\tau_{3}^{b}$.

\begin{tabular}{|c|c|c|c|c|c|c|c|}
\hline \multirow{2}{*}{ Molecule } & \multicolumn{2}{|c|}{ Short-time (fs-ns) } & \multicolumn{3}{c|}{ Long-time (ps- $\mu$ s) } & \multicolumn{2}{c|}{ Derived Constants } \\
\cline { 2 - 8 } & $\tau_{\mathbf{1}} / \mathbf{p s}$ & $\tau_{\mathbf{2}} / \mathbf{p s}$ & $\tau_{\mathbf{3}} / \mathbf{n s}$ & $\tau_{\mathbf{4}} / \mathbf{n s}$ & $\tau_{\mathbf{5}} / \mathbf{n s}$ & $\boldsymbol{\tau}_{\mathbf{3}}^{\boldsymbol{a}} / \mathbf{n s}$ & $\boldsymbol{\tau}_{\mathbf{3}}^{\boldsymbol{b}} / \mathbf{n s}$ \\
\hline $\mathbf{1 a}$ & $1.68 \pm 0.01$ & $228 \pm 1$ & $10.2 \pm 0.3$ & $60 \pm 1$ & $970 \pm 10$ & $39 \pm 1$ & $13.8 \pm 0.4$ \\
\hline $\mathbf{2 a}$ & $1.83 \pm 0.01$ & $230 \pm 1$ & $6.6 \pm 0.1$ & $64 \pm 1$ & $930 \pm 10$ & $21 \pm 1$ & $9.6 \pm 0.4$ \\
\hline 3a & $1.72 \pm 0.01$ & $88.2 \pm 0.1$ & $2.24 \pm 0.04^{\mathrm{a}}$ & $58 \pm 1$ & $950 \pm 10$ & $224 \pm 4$ & $2.26 \pm 0.4$ \\
\hline $\mathbf{1 b}$ & $1.31 \pm 0.01$ & $121.0 \pm 0.1$ & $9.8 \pm 0.3$ & $52 \pm 1$ & $850 \pm 10$ & $42 \pm 1$ & $12.7 \pm 0.4$ \\
\hline 2b & $2.31 \pm 0.01$ & $198.3 \pm 0.1$ & $18.1 \pm 0.4$ & $55 \pm 1$ & $780 \pm 10$ & $36.2 \pm 0.8$ & $36.2 \pm 0.8$ \\
\hline 3b & $2.05 \pm 0.01$ & $93.5 \pm 0.1$ & $9.0 \pm 0.3$ & $67 \pm 1$ & $840 \pm 10$ & $30 \pm 1$ & $12.9 \pm 0.4$ \\
\hline $\mathbf{1 c}$ & $1.61 \pm 0.01$ & $102.8 \pm 0.1$ & $3.3 \pm 0.3$ & $57 \pm 1$ & $850 \pm 10$ & $\mathrm{n} . \mathrm{a}$. & $3.3 \pm 0.3$ \\
\hline 2c & $1.93 \pm 0.01$ & $176.5 \pm 0.1$ & $5.2 \pm 0.3$ & $59 \pm 1$ & $790 \pm 10$ & $87 \pm 5$ & $5.5 \pm 0.3$ \\
\hline 3c & $1.52 \pm 0.01$ & $122.0 \pm 0.1$ & $3.4 \pm 0.3$ & $62 \pm 1$ & $910 \pm 10$ & $85 \pm 8$ & $3.5 \pm 0.3$ \\
\hline
\end{tabular}

${ }^{a}$ This time constant was obtained from short-time measurements and fixed in the global analysis of the long-time measurement. 

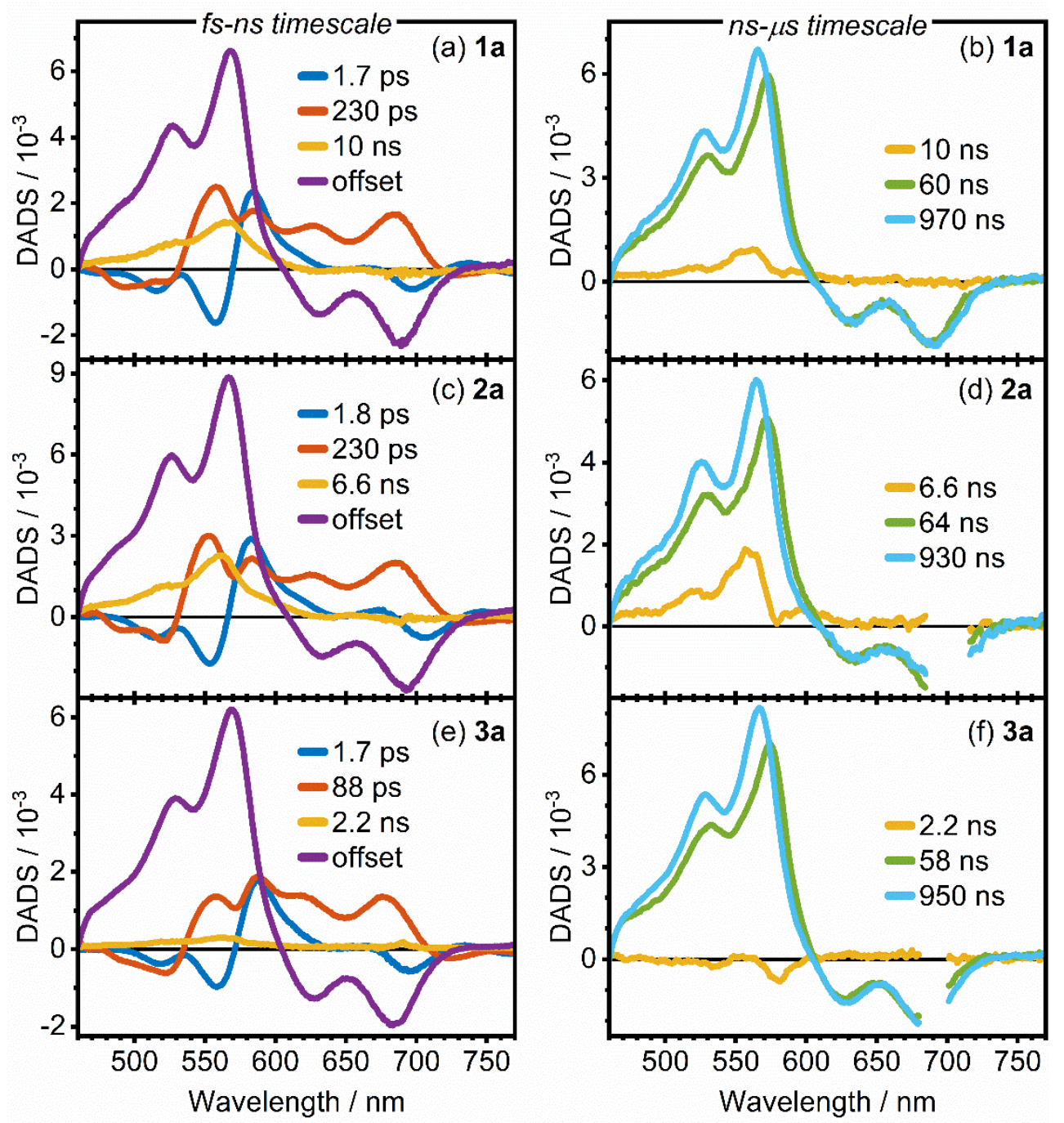

Figure S53: Decay-associated difference spectra (DADS) obtained by global analysis of TA measurements of DAP-dimers 1$3 a$. The left column $(a, c, e)$ shows the DADS of the short-time measurements whereas the right column $(b, d, f)$ shows the $D A D S$ of the long-time measurements for $(a, b) \mathbf{1 a},(c, d) \mathbf{2 a}$ and $(e, f) \mathbf{3 a}$, respectively. The corresponding time constants (c.f. Table S9) are given in the plot. 

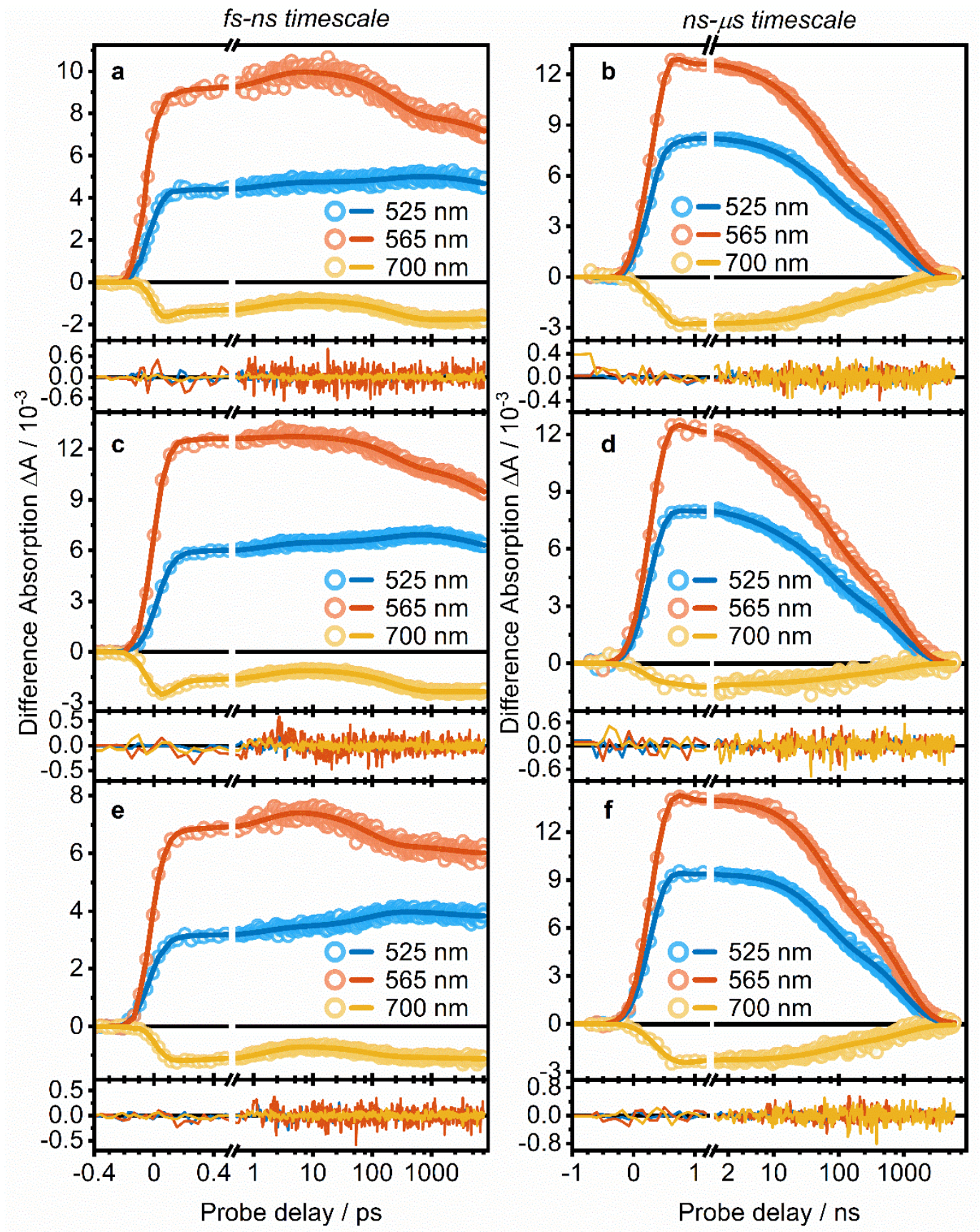

Figure S54: Fit of TA measurements of DAP-Dimers 1-3a. Kinetic traces at selected wavelengths and fit curves are shown in (a) and (b) for 1a, (c) and (d) for $2 \boldsymbol{a}$ and (e) and (f) for $3 \boldsymbol{a}$ for the $f s-n s-$ and $n s-\mu s$ time scale experiments, respectively. Residuals after subtraction of the fit are shown below the respective fit curves. Experimental data is shown as hollow circles, fit curves and residuals are shown as lines with the color coding for each selected wavelength shown in the legend. Notice the break after $0.5 \mathrm{ps}$ and $1.2 \mathrm{~ns}$ for the short-and long-timescale experiments, respectively, after which the probe delay is shown in a logarithmic scale. 

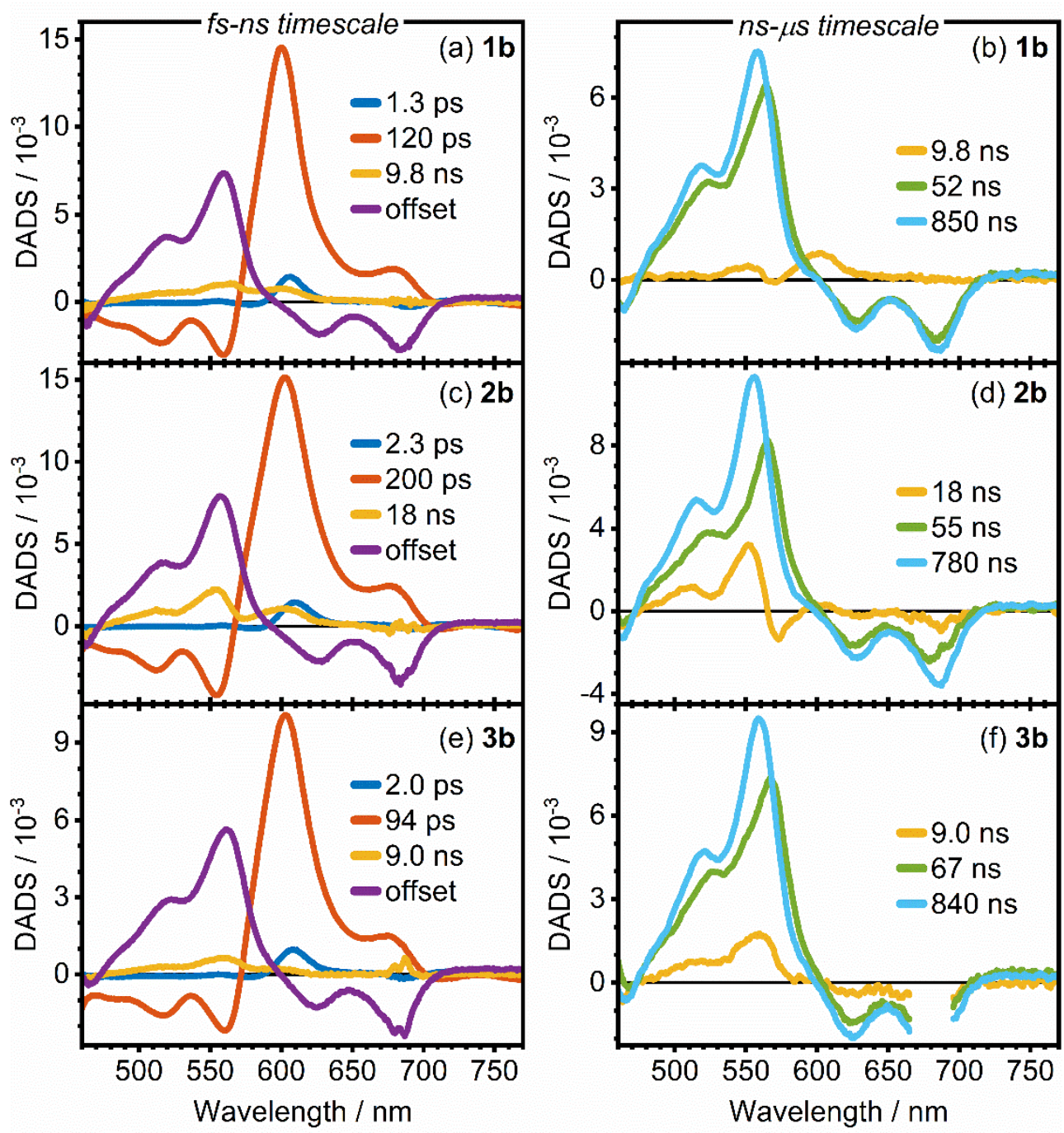

Figure S55: Decay-associated difference spectra (DADS) obtained by global analysis of TA measurements of DAP-dimers 1$3 \boldsymbol{b}$. The left column ( $a, c, e)$ shows the DADS of the short-time measurements whereas the right column ( $b, d, f)$ shows the $D A D S$ of the long-time measurements for $(a, b) \mathbf{1} \boldsymbol{b},(c, d) \mathbf{2} \boldsymbol{b}$ and $(e, f) \mathbf{3} \boldsymbol{b}$, respectively. The corresponding time constants (c.f. Table S9) are given in the plot. 

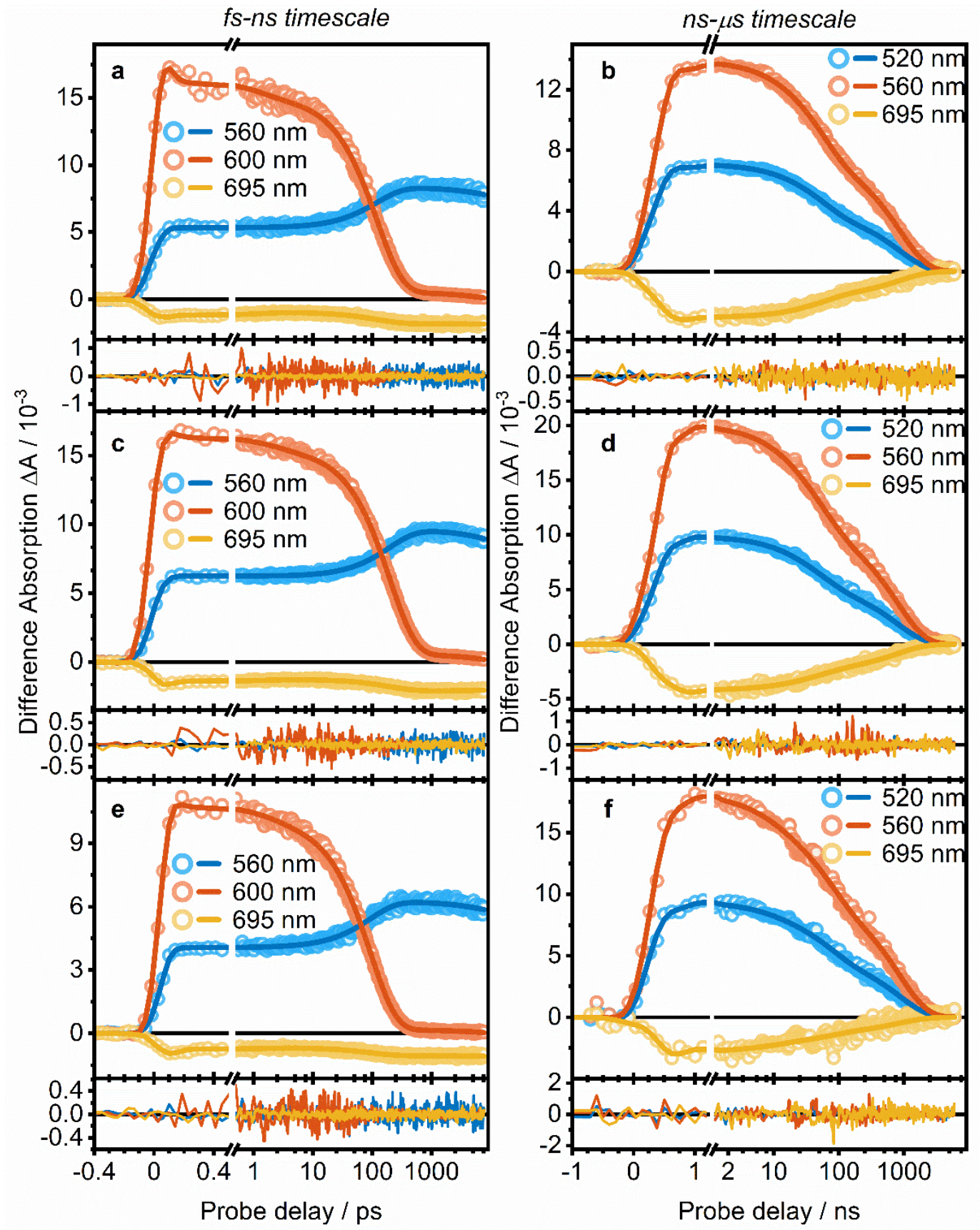

Figure S56: Fit of TA measurements of TAP-Dimers 1-3b. Kinetic traces at selected wavelengths and fit curves are shown in

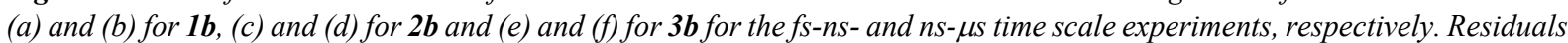
after subtraction of the fit are shown below the respective fit curves. Experimental data is shown as hollow circles, fit curves and residuals are shown as lines with the color coding for each selected wavelength shown in the legend. Notice the break after $0.5 \mathrm{ps}$ and $1.2 \mathrm{~ns}$ for the short- and long-timescale experiments, respectively, after which the probe delay is shown in a logarithmic scale. 

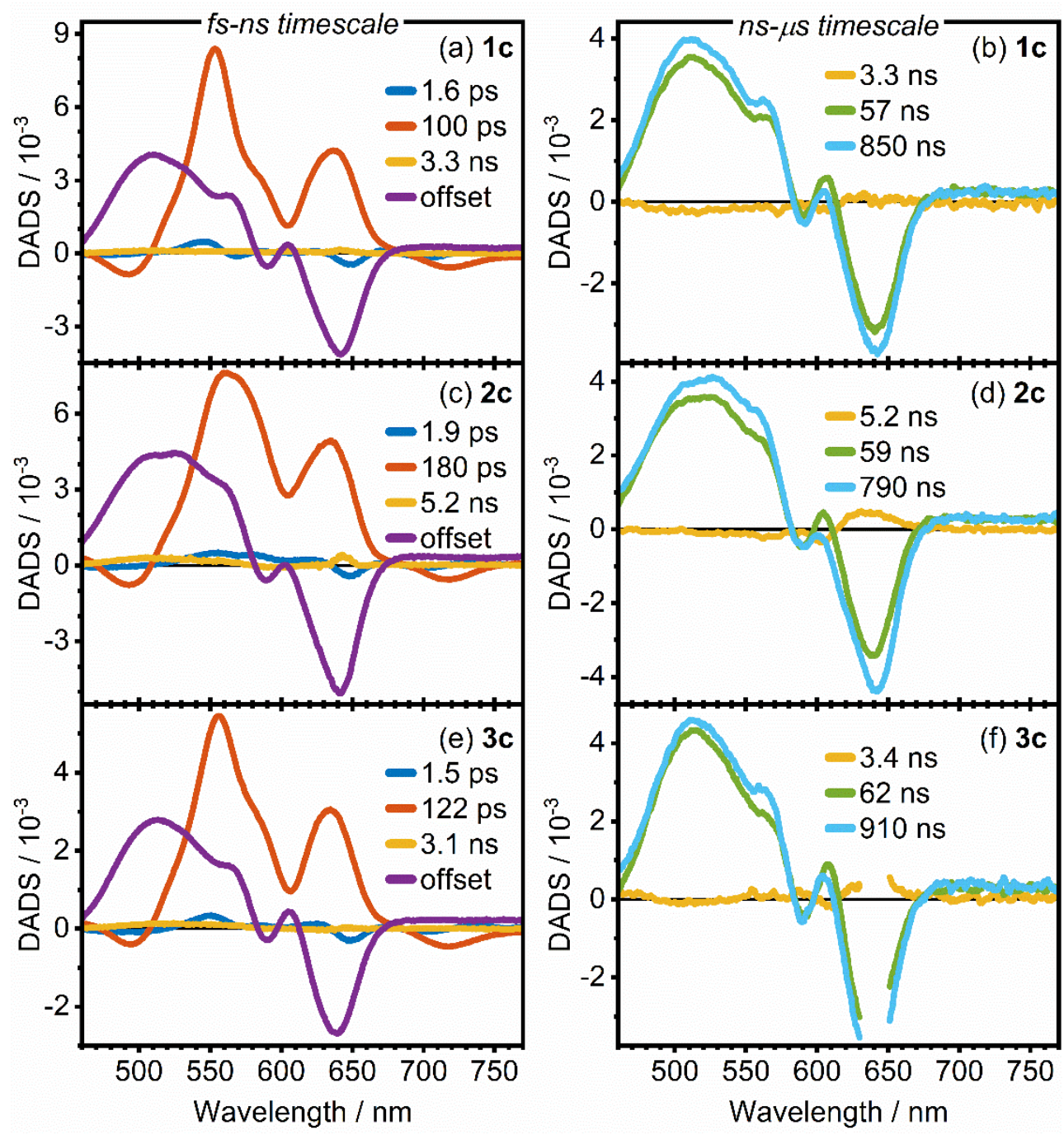

Figure S57: Decay-associated difference spectra (DADS) obtained by global analysis of TA measurements of DAP-dimers 1$3 c$. The left column $(a, c, e)$ shows the DADS of the short-time measurements whereas the right column $(b, d, f)$ shows the $D A D S$ of the long-time measurements for $(a, b) \mathbf{1 c},(c, d) 2 c$ and $(e, f) 3 c$, respectively. The corresponding time constants (c.f. Table S9) are given in the plot. 

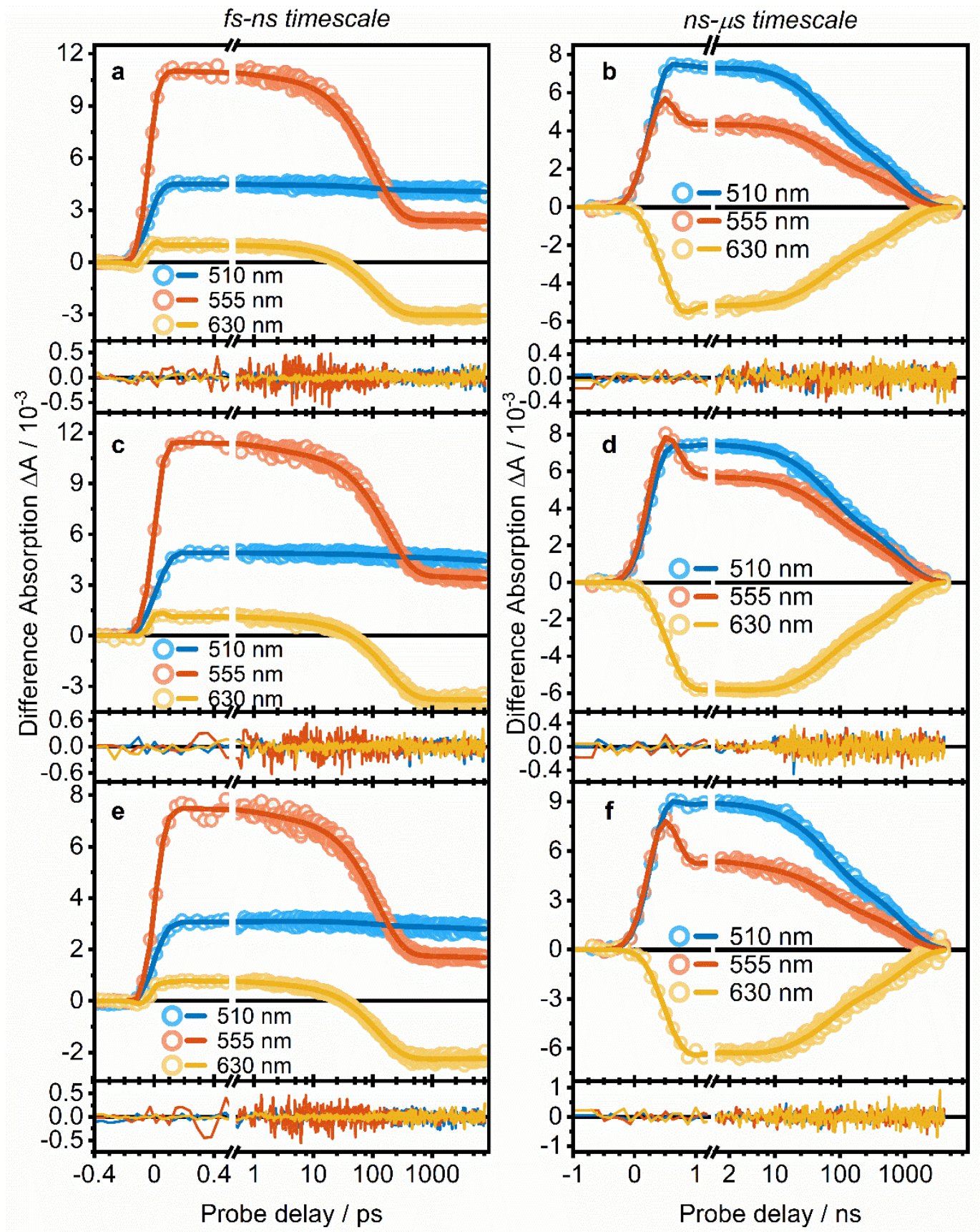

Figure S58: Fit of TA measurements of PTD-Dimers 1-3c. Kinetic traces at selected wavelengths and fit curves are shown in (a) and (b) for 1c, (c) and (d) for $2 c$ and (e) and (f) for $3 c$ for the $f s-n s-$ and $n s-\mu s$ time scale experiments, respectively. Residuals after subtraction of the fit are shown below the respective fit curves. Experimental data is shown as hollow circles, fit curves and residuals are shown as lines with the color coding for each selected wavelength shown in the legend. Notice the break after $0.5 \mathrm{ps}$ and $1.2 \mathrm{~ns}$ for the short-and long-timescale experiments, respectively, after which the probe delay is shown in a logarithmic scale. 


\subsubsection{Kinetic Models}

As stated in the main text, a sequential model adequately describes the evolution of singlet species until the formation of the correlated triplet pair denoted as ${ }^{1}(\mathrm{TT})$. Thus, we will focus on the SADS of the three proposed triplet species ${ }^{1}(\mathrm{TT}), \mathrm{T}_{1} \mathrm{~T}_{1}$ and $\mathrm{T}_{1} \mathrm{~S}_{0}$. Applying a sequential model (Figure S59a) results an identical spectral shape of all three triplet species, allowing for directly comparing their respective amplitudes and thus their populations. This is best illustrated in the case of 1c (PTD-unlinked, Figure S59h). Here, a sequential kinetic model results in identical SADS for ${ }^{1}(\mathrm{TT})$ and $\mathrm{T}_{1} \mathrm{~T}_{1}$, with $\mathrm{T}_{1} \mathrm{~S}_{0}$ exhibiting exactly half amplitude. This further indicates that within the triplet pair states, the constituting triplets are probed individually. The other PTD-containing homodimers exhibit deviations from this behaviour. Here, the $\mathrm{T}_{1} \mathrm{~S}_{0}$ SADS still possess half of the amplitude of the ${ }^{1}(\mathrm{TT})$, indicating the loss of half of the triplet population. However, the amplitude of $\mathrm{T}_{1} \mathrm{~T}_{1}$ is lower compared to ${ }^{1}(\mathrm{TT})$ and $2 \times \mathrm{T}_{1} \mathrm{~S}_{0}$. This would represent a loss of triplet population going from ${ }^{1}(\mathrm{TT})$ to $\mathrm{T}_{1} \mathrm{~T}_{1}$. Subsequently, TTA would have to result in an overall increase of triplet population. This is not physically feasible. Thus, a simple sequential model can be discarded.
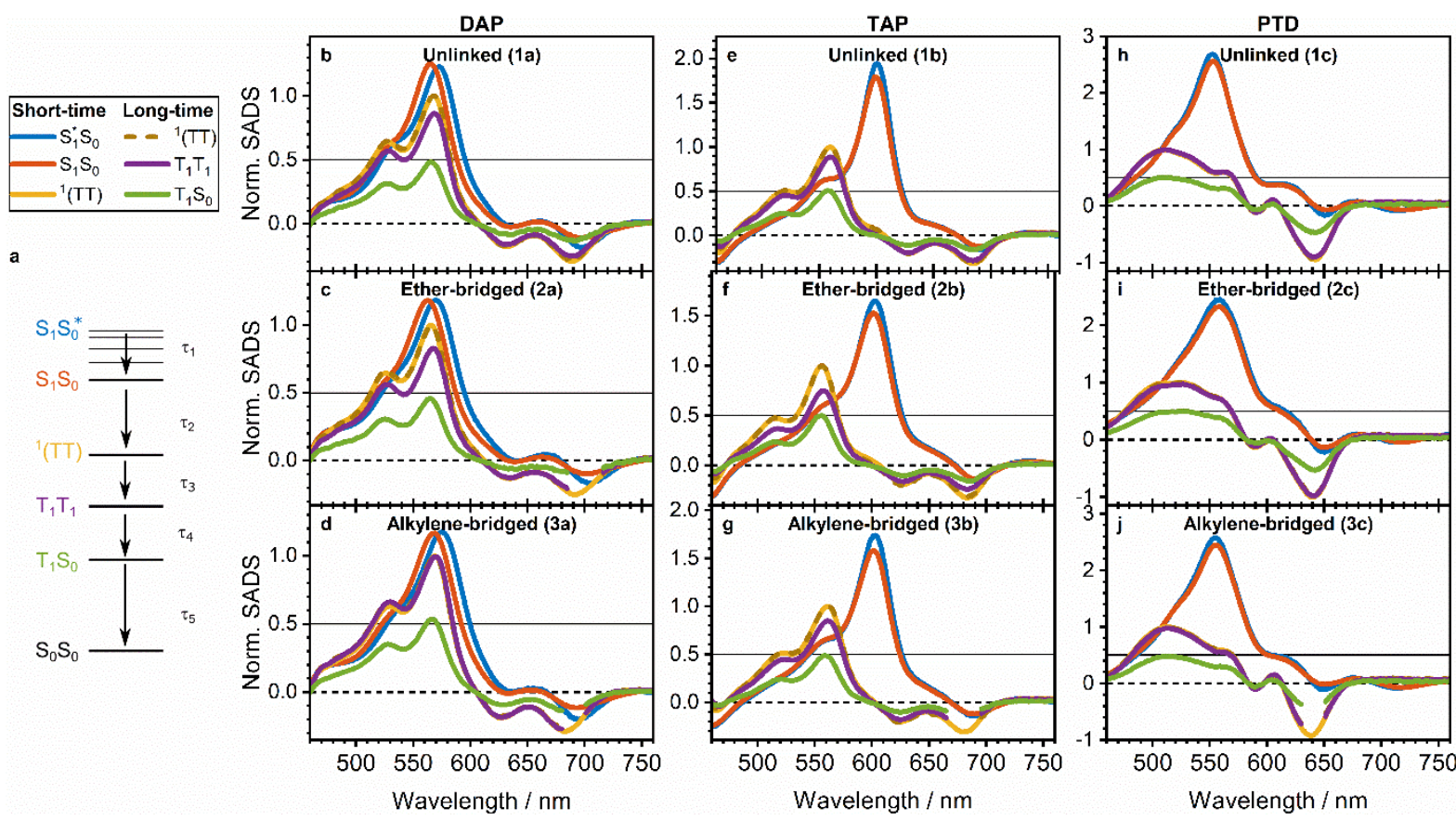

Figure S59: Combined SADS (b-j) of short-and long-time measurements obtained after applying a sequential kinetic model shown in (a). SADS of differently linked DAP dimers 1-3a are shown on the left (b-d), for the TAP Dimers $\mathbf{1 - 3 b}$ in the center (e-g) and for the PTD Dimers $1-3 c$ on the right $(h-j)$ with unlinked $1 \#(b, e, h)$, ether-bridged $2 \#(c, f, i)$ or alkylene-bridged $3 \#$ $(d, g, j)$ linker motif. 
Two other models including parasitic decay channels leading to $\mathrm{S}_{0} \mathrm{~S}_{0}$ are considered. The first possibility includes an additional decay pathway starting from ${ }^{1}(\mathrm{TT})$, which may include triplet fusion to $\mathrm{S}_{1} \mathrm{~S}_{0}$ (Model 1, Figure S60a). Both models are discussed using molecules $\mathbf{2 b}$ (TAP Ether bridged) and 3b (TAP - Alkylene bridged) as examples, since they exhibit the most pronounced deviation in a sequential model. As shown in Figure S60 b) and c), this does not result in the desired relation of amplitudes, that is ${ }^{1}(\mathrm{TT})=\mathrm{T}_{1} \mathrm{~T}_{1}=2 \mathrm{~T}_{1} \mathrm{~S}_{0}$. In this case, the amplitudes of $\mathrm{T}_{1} \mathrm{~S}_{0}$ do not match with their predecessors, despite the good agreement of the SADS of ${ }^{1}(\mathrm{TT})$ and $\mathrm{T}_{1} \mathrm{~T}_{1}$. On the other hand, a model with an additional decay channel of $\mathrm{T}_{1} \mathrm{~T}_{1}$ can also be considered (Model 2, Figure S60d). Here, the amplitudes of the resulting SADS do not match as well.
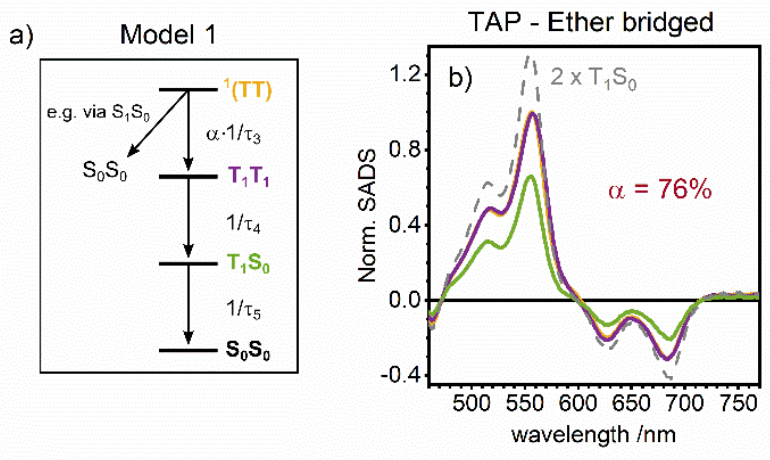

d)
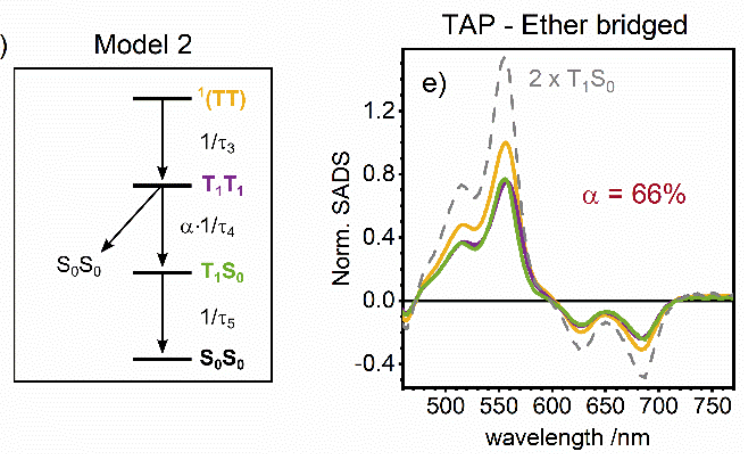
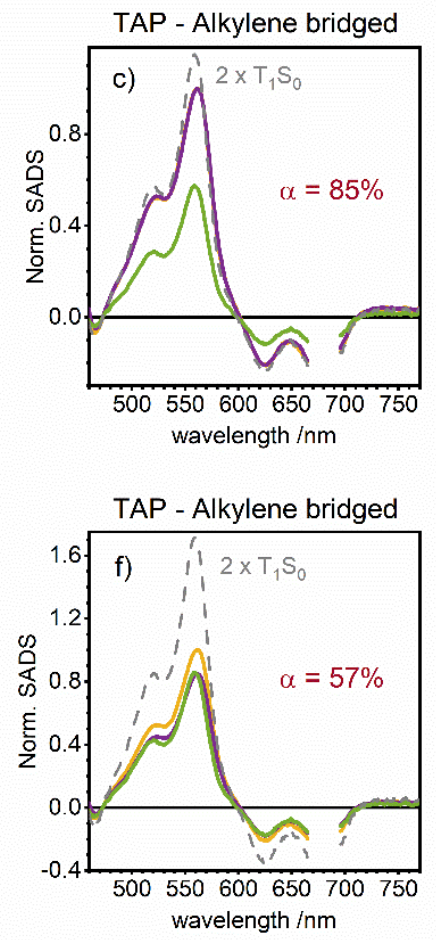

Figure S60 - Demonstration of different kinetic models, which may describe the dynamics of the spiroconjugated homodimers, representative for $2 b$ (TAP - Ether bridged, $b$ and e) and $3 b$ (TAP Alkylene bridged, $c$ and $f$ ). The first model in a includes a parasitic decay starting from ${ }^{1}(T T)$ with the respective normalized SADS shown in $b-c$. The second model includes a decay starting from $T_{1} T_{1}$ and the SADS are shown in e and $f$. The color scheme of the graphs is indicated in the kinetic models, where the colors of the different species in a and d correspond to the different spectra in $b-c$ and $e-f$, respectively. As reference, the grey dashed lines represent the spectra of $T_{1} S_{0}$ after multiplication of 2 . 
In contrast, the branching mechanism put forward in the main text and shown in Figure S61a yields SADS with physically meaningful amplitudes for all spiro-conjugated homodimers. Thus, we conclude that parasitic decay processes are not occurring or at least only by a negligible amount.
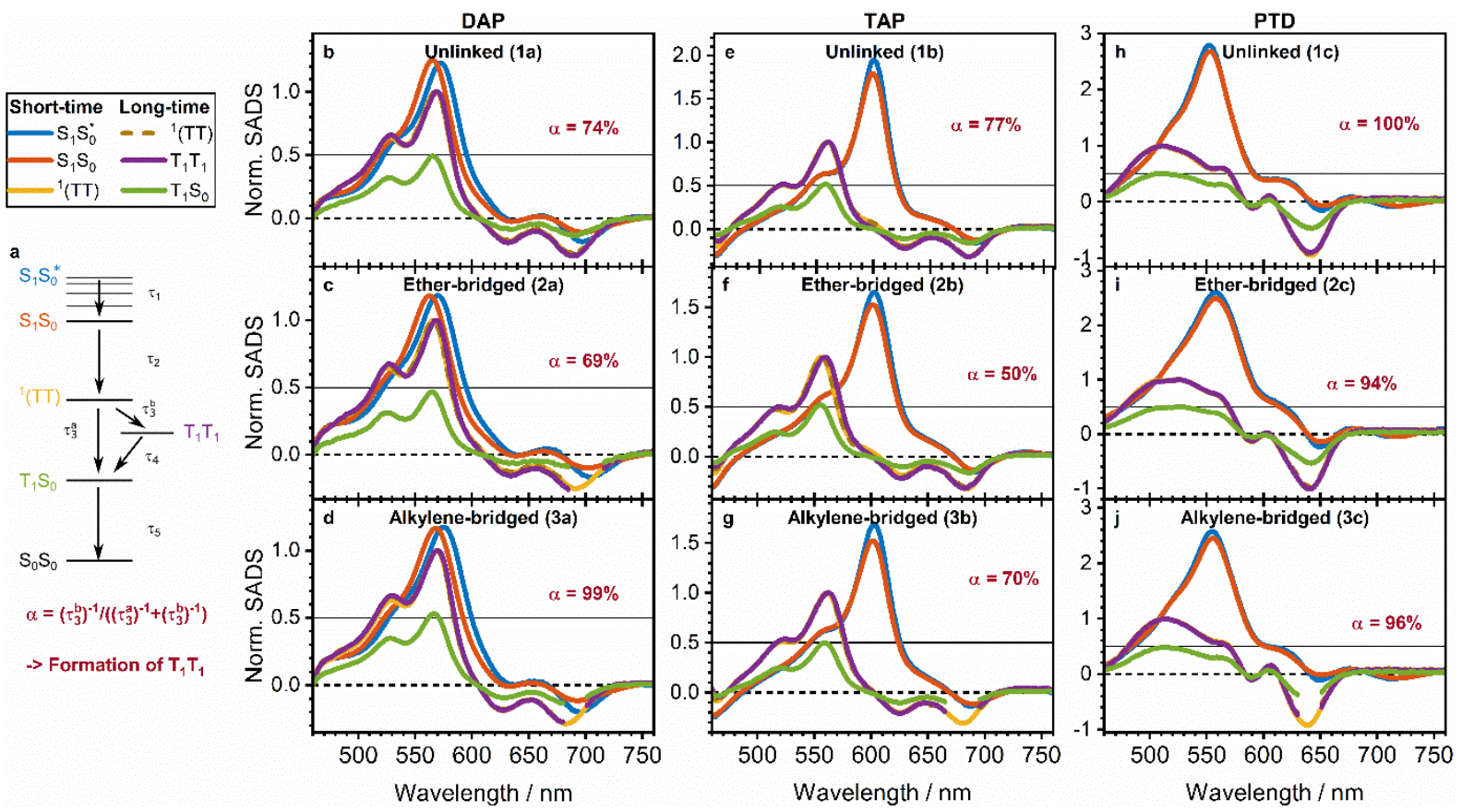

Figure S61: Combined SADS (b-j) of short- and long-time measurements obtained after applying a branched kinetic model shown in (a). SADS of differently linked DAP dimers 1-3a are shown on the left (b-d), for the TAP Dimers 1-3b in the center (e-g) and for the PTD Dimers 1-3c on the right (h-j) with unlinked $1 \#(b, e, h)$, ether-bridged $2 \#(c, f, i)$ or alkylene-bridged $3 \#$ $(d, g, j)$ linker motif. The values for the branching ratio $\alpha$ as depicted in (a) is shown in the respective plots. 


\subsubsection{Time-Resolved Emission}

Time-resolved emission traces of the dimers without a molecular brace $(1 \mathrm{x})$ and the corresponding monomers (8x) in Figure S62 show no delayed fluorescence signal, i.e. lifetime of ${ }^{1}(T T)$ is not observed. Fluorescence with slow decay constants contribute with a small amplitude and possess a lifetime similar to the monomeric compounds. This minor contribution is here assigned to residual amounts of contamination by e.g. unreacted compounds or (photo) degradation.
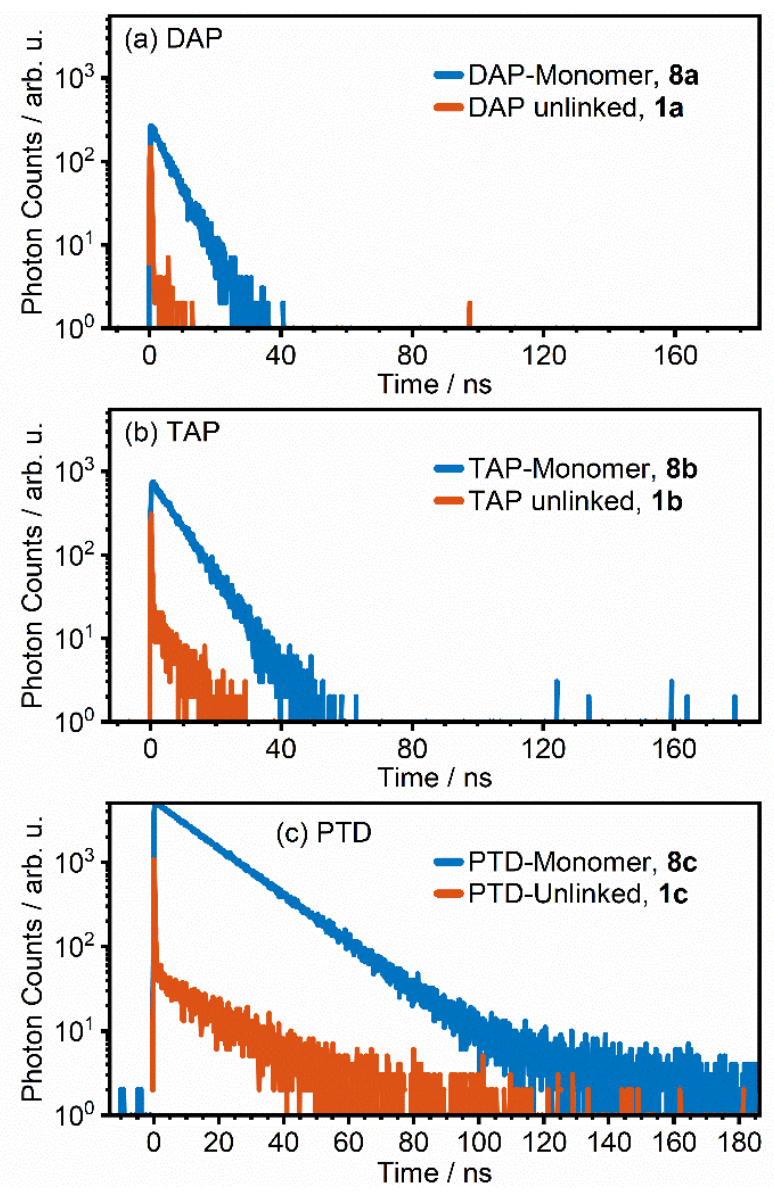

Figure S62: Comparison of Time-correlated single photon counting traces of Spiro-homodimers without a molecular brace $(\mathbf{1 x})$ and corresponding monomers (8x). Excitation wavelength was $635 \mathrm{~nm}$ for all compounds. Detection wavelength was $720 \mathrm{~nm}, 690 \mathrm{~nm}$ and $660 \mathrm{~nm}$ for (a) DAP-, (b) TAP- and (c) PTD-compounds, respectively. 


\section{Cartesian Coordinates of Optimized Geometries}

98

8a DAP-Monomer

H 8.544007000000

C 8.326040000000

C 7.196418000000

$\mathrm{H} \quad 8.059405000000$

H $\quad 9.251417000000$

H $\quad 6.283317000000$

C 6.924379000000

Si 7.467027000000

H 7.792095000000

H $\quad 6.072136000000$

H 6.701027000000

C 7.251832000000

C $\quad 6.098223000000$

C 9.185692000000

C 7.019954000000

H 6.202996000000

C 4.706603000000

C 6.235307000000

H 9.085716000000

C $\quad 10.300055000000$

C $\quad 9.565647000000$

C $\quad 6.754508000000$

H 4.563415000000

H 4.549995000000

H 3.919773000000

H 5.422566000000

H 7.180887000000

H 6.182118000000

H 10.479453000000

H $\quad 10.056527000000$

H 11.243705000000

H 10.488726000000

H 8.785054000000

H 9.744536000000

C $\quad 7.576972000000$

C $\quad 5.654890000000$

C $\quad 7.287674000000$

C 8.682618000000

C 5.365828000000

N 4.885093000000

C 8.120586000000

C $\quad 6.182925000000$

C 9.490027000000

N 4.313984000000

C $\quad 3.863291000000$

C $\quad 9.200089000000$

C 5.878949000000

C 10.616569000000

C 3.569831000000

C 3.018354000000

C 10.049154000000

C 5.622883000000

H 10.831618000000

C 11.392205000000

C 2.442330000000

C 1.964640000000

H 3.256479000000

C 11.103488000000

$\mathrm{H} \quad 9.826773000000$

Si $\quad 5.097587000000$

H 12.241075000000

H 2.239731000000

C $\quad 1.672790000000$

H 1.325229000000

H 11.739571000000

C $\quad 6.281966000000$

C $\quad 5.287023000000$

C 3.273322000000

$\mathrm{H} \quad 0.820976000000$

H 7.282987000000
$6.975372000000 \quad 2.521328000000$

$7.420731000000 \quad 1.543643000000$

$\begin{array}{ll}8.457290000000 & 1.622374000000\end{array}$

$6.606888000000 \quad 0.857508000000$

$7.872597000000 \quad 1.167248000000$

$7.927309000000 \quad 1.931645000000$

$9.077656000000 \quad 0.242938000000$

$9.751436000000 \quad 2.985996000000$

$9.634547000000 \quad-0.128098000000$

$9.765776000000 \quad 0.260745000000$

$8.294426000000-0.492627000000$

$8.889186000000 \quad 4.603446000000$

$11.064864000000 \quad 2.912553000000$

$10.558578000000 \quad 3.005936000000$

$8.394930000000 \quad 5.691535000000$

$11.577653000000 \quad 1.944544000000$

$10.414816000000 \quad 2.964140000000$

$12.113096000000 \quad 4.026068000000$

$11.393510000000 \quad 3.714882000000$

$9.646800000000 \quad 3.540635000000$

$1.152094000000 \quad 1.640728000000$

$7.811216000000 \quad 6.960403000000$

$9.880705000000 \quad 3.911527000000$

$9.698700000000 \quad 2.149575000000$

$11.176573000000 \quad 2.890723000000$

$12.848560000000 \quad 3.966840000000$

$12.662551000000 \quad 3.964717000000$

$11.641789000000 \quad 5.015157000000$

$8.795011000000 \quad 2.875366000000$

$9.249989000000 \quad 4.532078000000$

$10.201289000000 \quad 3.622585000000$

$11.740500000000 \quad 1.716205000000$

$11.810539000000 \quad 1.241617000000$

$10.361230000000 \quad 0.902551000000$

$6.776653000000 \quad 7.467783000000$

$8.275567000000 \quad 7.713912000000$

$6.196852000000 \quad 8.757858000000$

$6.293029000000 \quad 6.736851000000$

$7.696905000000 \quad 8.998528000000$

$9.267095000000 \quad 7.207988000000$

$5.166103000000 \quad 9.241569000000$

$6.665385000000 \quad 9.509603000000$

$5.281096000000 \quad 7.223682000000$

$8.126595000000 \quad 9.733920000000$

$9.679360000000 \quad 7.935534000000$

$4.700286000000 \quad 8.513252000000$

$6.106317000000 \quad 10.781307000000$

$4.784181000000 \quad 6.482210000000$

$9.093850000000 \quad 9.229717000000$

$10.731016000000 \quad 7.440700000000$

$3.648381000000 \quad 9.001595000000$

$5.621894000000 \quad 11.868478000000$

$5.224056000000 \quad 5.512293000000$

$3.787713000000 \quad 6.984057000000$

$9.583261000000 \quad 9.973957000000$

$11.160295000000 \quad 8.181381000000$

$11.155804000000 \quad 6.470901000000$

$3.209910000000 \quad 8.265042000000$

$3.213121000000 \quad 9.972005000000$

$4.972089000000 \quad 13.514155000000$

$3.418197000000 \quad 6.417173000000$

$9.131174000000 \quad 10.939446000000$

$10.578755000000 \quad 9.464054000000$

$11.954502000000 \quad 7.809558000000$

$2.414728000000 \quad 8.641242000000$

$5.700363000000 \quad 14.808841000000$

$3.084712000000 \quad 13.466688000000$

$5.462521000000 \quad 13.710821000000$

$10.949927000000 \quad 10.024907000000$

$5.548734000000 \quad 14.378175000000$ 


\begin{tabular}{|c|c|c|c|}
\hline $\mathrm{C}$ & 6.091651000000 & 7.209918000000 & 15.013583000000 \\
\hline $\mathrm{C}$ & 6.240203000000 & 4.956819000000 & 16.153178000000 \\
\hline $\mathrm{H}$ & 5.000880000000 & 2.716832000000 & 14.463421000000 \\
\hline $\mathrm{C}$ & 6.747577000000 & 2.681344000000 & 13.212769000000 \\
\hline $\mathrm{C}$ & 4.359173000000 & 2.428501000000 & 12.434708000000 \\
\hline $\mathrm{H}$ & 2.743565000000 & 4.812087000000 & 12.999260000000 \\
\hline $\mathrm{C}$ & 2.964254000000 & 6.912443000000 & 13.306900000000 \\
\hline $\mathrm{C}$ & 2.734933000000 & 5.150022000000 & 15.115253000000 \\
\hline $\mathrm{H}$ & 6.889794000000 & 7.619653000000 & 15.645544000000 \\
\hline $\mathrm{H}$ & 6.100144000000 & 7.758084000000 & 14.064736000000 \\
\hline $\mathrm{H}$ & 5.139676000000 & 7.422261000000 & 15.514423000000 \\
\hline $\mathrm{H}$ & 5.259591000000 & 5.046767000000 & 16.634193000000 \\
\hline $\mathrm{H}$ & 6.457995000000 & 3.888968000000 & 16.042008000000 \\
\hline $\mathrm{H}$ & 6.982158000000 & 5.374510000000 & 16.845637000000 \\
\hline $\mathrm{H}$ & 7.085586000000 & 3.037360000000 & 12.231308000000 \\
\hline $\mathrm{H}$ & 7.429293000000 & 3.089864000000 & 13.967157000000 \\
\hline $\mathrm{H}$ & 6.858042000000 & 1.589611000000 & 13.221938000000 \\
\hline $\mathrm{H}$ & 4.513252000000 & 1.342173000000 & 12.409272000000 \\
\hline $\mathrm{H}$ & 3.302169000000 & 2.606158000000 & 12.659838000000 \\
\hline $\mathrm{H}$ & 4.556241000000 & 2.812906000000 & 11.426184000000 \\
\hline $\mathrm{H}$ & 3.426323000000 & 7.629026000000 & 13.994970000000 \\
\hline $\mathrm{H}$ & 3.326382000000 & 7.138290000000 & 12.297953000000 \\
\hline $\mathrm{H}$ & 1.881066000000 & 7.091595000000 & 13.329452000000 \\
\hline $\mathrm{H}$ & 1.649137000000 & 5.302762000000 & 15.158481000000 \\
\hline $\mathrm{H}$ & 2.935792000000 & 4.115114000000 & 15.417094000000 \\
\hline $\mathrm{H}$ & 3.185378000000 & 5.809938000000 & 15.866359000000 \\
\hline $\mathrm{H}$ & 8.897763000000 & 6.731501000000 & 5.766625000000 \\
\hline $\mathrm{H}$ & 7.901383000000 & 4.732460000000 & 10.212855000000 \\
\hline \multicolumn{4}{|c|}{96} \\
\hline \multicolumn{4}{|c|}{ 8b_TAP-Monomer } \\
\hline $\mathrm{H}$ & 7.254728000000 & 6.799503000000 & 2.366919000000 \\
\hline $\mathrm{C}$ & 7.144856000000 & 7.352819000000 & 1.427416000000 \\
\hline $\mathrm{C}$ & 6.594862000000 & 8.768246000000 & 1.652443000000 \\
\hline $\mathrm{H}$ & 6.477252000000 & 6.776439000000 & 0.774409000000 \\
\hline $\mathrm{H}$ & 8.126785000000 & 7.384824000000 & 0.940628000000 \\
\hline $\mathrm{H}$ & 5.580406000000 & 8.667349000000 & 2.065746000000 \\
\hline $\mathrm{C}$ & 6.477974000000 & 9.527431000000 & 0.321317000000 \\
\hline $\mathrm{Si}$ & 7.548634000000 & 9.736067000000 & 2.980347000000 \\
\hline $\mathrm{H}$ & 7.459107000000 & 9.687997000000 & -0.139987000000 \\
\hline $\mathrm{H}$ & 6.005562000000 & 10.507982000000 & 0.445289000000 \\
\hline $\mathrm{H}$ & 5.871345000000 & 8.956917000000 & -0.393542000000 \\
\hline $\mathrm{C}$ & 7.143581000000 & 8.971506000000 & 4.609490000000 \\
\hline $\mathrm{C}$ & 6.913241000000 & 11.522822000000 & 3.048682000000 \\
\hline $\mathrm{C}$ & 9.439958000000 & 9.688497000000 & 2.819646000000 \\
\hline $\mathrm{C}$ & 6.899504000000 & 8.485415000000 & 5.697689000000 \\
\hline $\mathrm{H}$ & 7.107505000000 & 11.967095000000 & 2.061031000000 \\
\hline $\mathrm{C}$ & 5.396761000000 & 11.552892000000 & 3.296919000000 \\
\hline $\mathrm{C}$ & 7.648908000000 & 12.364031000000 & 4.101042000000 \\
\hline $\mathrm{H}$ & 9.790572000000 & 10.428249000000 & 3.554435000000 \\
\hline $\mathrm{C}$ & 10.069545000000 & 8.340733000000 & 3.204809000000 \\
\hline $\mathrm{C}$ & 9.917814000000 & 10.147489000000 & 1.433732000000 \\
\hline $\mathrm{C}$ & 6.621039000000 & 7.913180000000 & 6.966345000000 \\
\hline $\mathrm{H}$ & 5.150196000000 & 11.091862000000 & 4.261484000000 \\
\hline $\mathrm{H}$ & 4.839454000000 & 11.016749000000 & 2.520353000000 \\
\hline $\mathrm{H}$ & 5.026217000000 & 12.585751000000 & 3.317510000000 \\
\hline $\mathrm{H}$ & 7.239402000000 & 13.381553000000 & 4.140217000000 \\
\hline $\mathrm{H}$ & 8.719707000000 & 12.447743000000 & 3.886141000000 \\
\hline $\mathrm{H}$ & 7.540106000000 & 11.926730000000 & 5.101308000000 \\
\hline $\mathrm{H}$ & 9.805541000000 & 7.554419000000 & 2.489075000000 \\
\hline $\mathrm{H}$ & 9.744744000000 & 8.009169000000 & 4.197021000000 \\
\hline $\mathrm{H}$ & 11.164518000000 & 8.419188000000 & 3.213818000000 \\
\hline $\mathrm{H}$ & 11.010238000000 & 10.248901000000 & 1.412163000000 \\
\hline $\mathrm{H}$ & 9.489694000000 & 11.115149000000 & 1.146104000000 \\
\hline $\mathrm{H}$ & 9.644296000000 & 9.418963000000 & 0.661031000000 \\
\hline $\mathrm{C}$ & 7.489344000000 & 6.943578000000 & 7.516460000000 \\
\hline $\mathrm{C}$ & 5.475613000000 & 8.307319000000 & 7.693535000000 \\
\hline $\mathrm{C}$ & 7.208539000000 & 6.360843000000 & 8.802657000000 \\
\hline $\mathrm{N}$ & 8.584421000000 & 6.578006000000 & 6.811449000000 \\
\hline $\mathrm{C}$ & 5.194800000000 & 7.724566000000 & 8.979725000000 \\
\hline $\mathrm{N}$ & 4.654370000000 & 9.239243000000 & 7.157471000000 \\
\hline $\mathrm{N}$ & 8.029805000000 & 5.428958000000 & 9.338741000000 \\
\hline $\mathrm{C}$ & 6.063107000000 & 6.754966000000 & 9.529837000000 \\
\hline $\mathrm{C}$ & 9.379266000000 & 5.669088000000 & 7.345359000000 \\
\hline $\mathrm{N}$ & 4.099714000000 & 8.090134000000 & 9.684731000000 \\
\hline $\mathrm{C}$ & 3.590193000000 & 9.589600000000 & 7.856237000000 \\
\hline $\mathrm{C}$ & 9.093985000000 & 5.078598000000 & 8.639978000000 \\
\hline $\mathrm{C}$ & 5.784690000000 & 6.182780000000 & 10.798525000000 \\
\hline
\end{tabular}




\begin{tabular}{|c|c|c|c|}
\hline $\mathrm{C}$ & 10.554739000000 & 5.250123000000 & 6.633762000000 \\
\hline $\mathrm{C}$ & 3.304881000000 & 8.999063000000 & 9.150829000000 \\
\hline $\mathrm{C}$ & 2.689005000000 & 10.576621000000 & 7.329415000000 \\
\hline $\mathrm{C}$ & 9.995198000000 & 4.091626000000 & 9.166841000000 \\
\hline $\mathrm{C}$ & 5.540591000000 & 5.696735000000 & 11.886739000000 \\
\hline $\mathrm{H}$ & 10.749328000000 & 5.705168000000 & 5.668273000000 \\
\hline $\mathrm{C}$ & 11.376702000000 & 4.314520000000 & 7.173928000000 \\
\hline $\mathrm{C}$ & 2.129389000000 & 9.417997000000 & 9.862419000000 \\
\hline $\mathrm{C}$ & 1.591142000000 & 10.940583000000 & 8.040148000000 \\
\hline $\mathrm{H}$ & 2.920795000000 & 11.006926000000 & 6.360851000000 \\
\hline $\mathrm{C}$ & 11.093051000000 & 3.727639000000 & 8.456103000000 \\
\hline $\mathrm{H}$ & 9.763422000000 & 3.661378000000 & 10.135435000000 \\
\hline $\mathrm{Si}$ & 5.135688000000 & 4.932198000000 & 13.515923000000 \\
\hline $\mathrm{H}$ & 12.265236000000 & 3.996180000000 & 6.638320000000 \\
\hline $\mathrm{H}$ & 1.934772000000 & 8.962898000000 & 10.827875000000 \\
\hline $\mathrm{C}$ & 1.307446000000 & 10.353633000000 & 9.322282000000 \\
\hline $\mathrm{H}$ & 0.908844000000 & 11.685753000000 & 7.644022000000 \\
\hline $\mathrm{H}$ & 11.775371000000 & 2.982502000000 & 8.852252000000 \\
\hline $\mathrm{C}$ & 6.089617000000 & 5.899932000000 & 14.843761000000 \\
\hline $\mathrm{C}$ & 5.771008000000 & 3.145400000000 & 13.447458000000 \\
\hline $\mathrm{C}$ & 3.244395000000 & 4.979681000000 & 13.676890000000 \\
\hline $\mathrm{H}$ & 0.418900000000 & 10.671950000000 & 9.857883000000 \\
\hline $\mathrm{H}$ & 7.104033000000 & 6.000845000000 & 14.430361000000 \\
\hline $\mathrm{C}$ & 5.539671000000 & 7.315358000000 & 15.068939000000 \\
\hline $\mathrm{C}$ & 6.206629000000 & 5.140668000000 & 16.174831000000 \\
\hline $\mathrm{H}$ & 5.576744000000 & 2.701108000000 & 14.435106000000 \\
\hline $\mathrm{C}$ & 7.287472000000 & 3.115234000000 & 13.199144000000 \\
\hline $\mathrm{C}$ & 5.035273000000 & 2.304251000000 & 12.395102000000 \\
\hline $\mathrm{H}$ & 2.893733000000 & 4.239964000000 & 12.942091000000 \\
\hline $\mathrm{C}$ & 2.614666000000 & 6.327418000000 & 13.291889000000 \\
\hline $\mathrm{C}$ & 2.766736000000 & 4.520549000000 & 15.062825000000 \\
\hline $\mathrm{H}$ & 6.207361000000 & 7.891678000000 & 15.721910000000 \\
\hline $\mathrm{H}$ & 5.429702000000 & 7.868747000000 & 14.129490000000 \\
\hline $\mathrm{H}$ & 4.557797000000 & 7.283342000000 & 15.555835000000 \\
\hline $\mathrm{H}$ & 5.225541000000 & 4.980059000000 & 16.636212000000 \\
\hline $\mathrm{H}$ & 6.679053000000 & 4.160137000000 & 16.050768000000 \\
\hline $\mathrm{H}$ & 6.813307000000 & 5.711162000000 & 16.889665000000 \\
\hline $\mathrm{H}$ & 7.533985000000 & 3.576221000000 & 12.234553000000 \\
\hline $\mathrm{H}$ & 7.844871000000 & 3.651360000000 & 13.975657000000 \\
\hline $\mathrm{H}$ & 7.657953000000 & 2.082353000000 & 13.178550000000 \\
\hline $\mathrm{H}$ & 5.444747000000 & 1.286718000000 & 12.355884000000 \\
\hline $\mathrm{H}$ & 3.964478000000 & 2.220560000000 & 12.610033000000 \\
\hline $\mathrm{H}$ & 5.144054000000 & 2.741580000000 & 11.394845000000 \\
\hline $\mathrm{H}$ & 2.878710000000 & 7.113723000000 & 14.007614000000 \\
\hline $\mathrm{H}$ & 2.939287000000 & 6.659038000000 & 12.299640000000 \\
\hline $\mathrm{H}$ & 1.519698000000 & 6.248898000000 & 13.283027000000 \\
\hline $\mathrm{H}$ & 1.674327000000 & 4.419007000000 & 15.084493000000 \\
\hline $\mathrm{H}$ & 3.194999000000 & 3.552922000000 & 15.350347000000 \\
\hline $\mathrm{H}$ & 3.040251000000 & 5.249056000000 & 15.835543000000 \\
\hline \multicolumn{4}{|l|}{98} \\
\hline \multicolumn{4}{|c|}{ 8c_PTD-Monomer } \\
\hline $\mathrm{H}$ & 5.781240000000 & -3.288756000000 & -0.566230000000 \\
\hline $\mathrm{H}$ & 5.194809000000 & 2.571315000000 & -2.128760000000 \\
\hline $\mathrm{Si}$ & 5.898251000000 & 0.501786000000 & -0.029657000000 \\
\hline $\mathrm{Si}$ & -5.946991000000 & 0.407536000000 & 0.011849000000 \\
\hline $\mathrm{C}$ & 2.855153000000 & 0.770953000000 & -0.102509000000 \\
\hline $\mathrm{C}$ & 4.072093000000 & 0.771087000000 & -0.097201000000 \\
\hline $\mathrm{C}$ & -2.886061000000 & 0.518098000000 & -0.008837000000 \\
\hline $\mathrm{C}$ & -4.102097000000 & 0.484226000000 & 0.017786000000 \\
\hline $\mathrm{C}$ & 1.439454000000 & 0.716248000000 & -0.090466000000 \\
\hline $\mathrm{C}$ & 0.645507000000 & 1.866398000000 & -0.269088000000 \\
\hline $\mathrm{N}$ & 1.107377000000 & 3.116145000000 & -0.474855000000 \\
\hline S & -0.189895000000 & 4.059666000000 & -0.614630000000 \\
\hline $\mathrm{N}$ & -1.383819000000 & 2.994893000000 & -0.431078000000 \\
\hline $\mathrm{C}$ & -0.796289000000 & 1.795997000000 & -0.243267000000 \\
\hline $\mathrm{C}$ & -1.470711000000 & 0.574951000000 & -0.038505000000 \\
\hline $\mathrm{C}$ & -0.678896000000 & -0.581043000000 & 0.134784000000 \\
\hline $\mathrm{N}$ & -1.309397000000 & -1.765274000000 & 0.327055000000 \\
\hline $\mathrm{C}$ & -0.563902000000 & -2.840169000000 & 0.490722000000 \\
\hline $\mathrm{C}$ & -1.191644000000 & -4.116852000000 & 0.697226000000 \\
\hline $\mathrm{H}$ & -2.275859000000 & -4.150315000000 & 0.710825000000 \\
\hline $\mathrm{C}$ & -0.429778000000 & -5.226716000000 & 0.867305000000 \\
\hline $\mathrm{H}$ & -0.901301000000 & -6.191561000000 & 1.023626000000 \\
\hline $\mathrm{C}$ & 1.007965000000 & -5.154460000000 & 0.845302000000 \\
\hline $\mathrm{H}$ & 1.578699000000 & -6.066713000000 & 0.985748000000 \\
\hline $\mathrm{C}$ & 1.647835000000 & -3.973593000000 & 0.653240000000 \\
\hline $\mathrm{H}$ & 2.729996000000 & -3.894934000000 & 0.632862000000 \\
\hline
\end{tabular}


C - $2.4572880 .091587-2.591273$ $\mathrm{N}-1.4703381 .012918-2.479967$ C - $1.7862192 .216715-2.045487$ C $-0.7180373 .155677-1.758622$ C - $1.0623164 .310641-1.119592$ C - 1.1168276 .1530760 .443671 H - 1.1782685 .7840561 .472384 H - 0.7782687 .1917830 .500452 C - $2.5088816 .045374-0.229396$ C $-2.4375284 .685375-0.892643$ C - $3.4638613 .864699-1.215716$ H - $4.5040524 .108314-1.023004$ C -3.165349 2.565810 -1.745228 N $-4.1397081 .691262-1.888512$ C $-3.8144500 .436073-2.279700$ C $-4.810480-0.560421-2.257296$ C $-4.500775-1.883309-2.652781$ C $-5.480666-2.895622-2.647600$ H $-6.500741-2.632994-2.380801$ C $-5.173355-4.206503-2.968698$ C $-6.166373-5.244626-2.965438$ H -7.189301 -4.982177 -2.709898 C $-5.832429-6.525627-3.274081$ H $-6.589285-7.303724-3.267643$ C $-4.481337-6.868183-3.610918$ H $-4.242283-7.899807-3.849996$ C $-3.510145-5.917636-3.630888$ H $-2.484708-6.172171-3.884477$ C $-3.814664-4.550224-3.311694$ C $-2.842231-3.565049-3.316281$ H $-1.817579-3.825260-3.565007$ C $-3.140885-2.225847-2.994754$ C $-2.6775337 .120402-1.320348$ H -2.646368 8.120169-0.872931 H $-1.8864927 .057136-2.075047$ H -3.638550 6.998733 - 1.831268 C -3.6419116 .1608100 .792338$ $\mathrm{H}-3.5858687 .1244591 .310281$ H -4.622748 6.0998620 .308136 H -3.581631 5.3634651 .541217 C $0.6863962 .696447-2.030641$ H $1.4026763 .517718-2.033605$ H $0.7379462 .177958-2.990823$ H $1.0069101 .979760-1.267491$ C $2.035027-0.8788402 .651916$ C 2.3617730 .4413452 .284131 N 1.3611451 .3409722 .151499 C 1.6439582 .5086701 .613260 C 0.5398573 .3710071 .243560 C 0.8383464 .4521280 .470211 C $0.8172276 .095072-1.297280$ H $0.9072675 .609057-2.273984$ H $0.4288167 .101478-1.479303$ C $2.2059116 .137106-0.608680$ C 2.1981484 .8543050 .199118 C 3.2581374 .1144760 .603627 H 4.2868324 .3750230 .375008 C 3.0096972 .8628401 .261604 N 4.0076262 .0241281 .463253 C 3.7079570 .7868211 .929591 C $4.711234-0.2010251 .936652$ C $4.395209-1.5335912 .304225$ C $5.366971-2.5537052 .261102$ H $6.385282-2.2928161 .987291$ C $5.050427-3.8726232 .536702$ C $6.029314-4.9206452 .458280$ H $7.050715-4.6567772 .197709$ C $5.682565-6.2130962 .697210$ H $6.427943-6.9994892 .630904$ C $4.332603-6.5570303 .037688$ H 4.083289 -7.597658 3.220545 C $3.375735-5.5962423 .133089$ H $2.351170-5.8512003 .390044$ C $3.694278-4.2174842 .886063$ C $2.734133-3.2236592 .949104$ H $1.709196-3.4826543 .197920$ C $3.040170-1.8780202 .663176$ 
C 2.3021377 .3289740 .363319 H $2.2169758 .273763-0.185212$ H 1.510413 7.298586 1.119085 H 3.2641417 .3179100 .886628 C $3.3433766 .206590-1.628779$ H $3.2443897 .107798-2.243659$ H $4.3196896 .247786-1.133614$ H $3.3339255 .334937-2.292361$ C -0.8425082 .8838461 .573725$ H - 0.8627742 .4659522 .582578 $\mathrm{H}-1.1353512 .0763530 .893801$ H - 1.5944293 .6691691 .499589 C $2.884278-3.217856-4.296672$ H $3.892514-3.576736-4.039625$ C $3.023809-2.142152-5.384241$ H $3.672529-1.318096-5.067778$ H $3.450315-2.567637-6.301507$ H $2.046648-1.715193-5.642227$ C $2.071528-4.412551-4.815987$ H 2.020719 -5.225999 -4.084142 H $1.042990-4.113459-5.054215$ H $2.517144-4.820156-5.732378$ C $2.092364-3.819733-1.335477$ H $1.826604-4.751252-1.857078$ C $3.469448-4.032984-0.686910$ H $4.262909-4.188286-1.428036$ H $3.752473-3.172492-0.070468$ H $3.456298-4.907261-0.023684$ C $1.016398-3.578471-0.265588$ H $0.016216-3.536768-0.708710$ H $1.027788-4.3850950 .479044$ H $1.177702-2.6387950 .274222$ C $3.036766-0.894623-2.217682$ H $2.788840-0.185392-3.021573$ C $4.567060-1.003326-2.140527$ H $5.007942-1.394597-3.064920$ H $5.008094-0.016662-1.949617$ H $4.883851-1.654818-1.319046$ C $2.476266-0.332270-0.908203$ H $1.384622-0.241251-0.927114$ H $2.741136-0.982868-0.068538$ H $2.9000890 .658599-0.701523$ C $-9.283503-0.4064850 .979964$ H -9.8067470 .0343621 .841268$ C $-10.325373-0.695386-0.109665$ $\mathrm{H}-10.8927840 .200693-0.383885$ H -9.849257 -1.081639-1.019763 H $-11.045125-1.4510530 .230134$ C -8.609514 -1.705945 1.448011 H -7.903317 - 1.5345782 .268619 H $-9.356601-2.4296061 .797822$ H -8.053441 -2.175191 0.626798 C -8.819361 $2.411833-0.294500$ H -9.551616 2.018389-1.015334 C -9.5921843 .1971460 .776042$ $\mathrm{H}-10.2872132 .5624101 .338273$ H -8.905349 3.658448 1.495948 $\mathrm{H}-10.1760234 .0067840 .320218$ C -7.868043 3.333881-1.072357 H -7.281601 2.785908 -1.816947 $\mathrm{H}-8.4326394 .118774-1.591817$ H -7.161022 $3.832502-0.400197$ C -6.745064 1.170566 1.826566 $\mathrm{H}-6.1894950 .2222741 .896223$ C - 7.4207711 .4147683 .184582 $\mathrm{H}-8.0767920 .5871543 .475830$ H -6.666806 1.5305283 .973634 H -8.024337 2.329739 3.175378 C -5.7246482 .2743781 .515114$ H -5.250968 2.130318 0.537787 H -6.199331 3.2629681 .522629 H -4.931526 2.292343 2.274946 C $-3.224463-1.5499141 .722451$ H -4.292273 -1.627845 1.979010 C - $2.981568-0.1505341 .142263$ H -3.151457 0.645039 1.875354 H - $1.949493-0.0571000 .782268$ 
H -3.6538000 .0385980 .298599$ C - $2.913188-2.6250880 .672907$ H -3.154037 -3.634060 1.025436 H -3.490749 -2.449104 -0.241412 H - $1.850972-2.6078750 .398696$ C $-2.437671-3.6386873 .888783$ H - $2.033933-4.2222243 .048563$ C -3.898734 -4.062306 4.099932 H $-4.523845-3.8444523 .225916$ $\mathrm{H}-3.969414-5.1394154 .297305$ H -4.338103 -3.546188 4.962327 C $-1.588931-3.9601495 .126293$ H $-0.534403-3.7049374 .973253$ H - $1.940990-3.4084296 .006749$ H - $1.644722-5.0290715 .369003$ C - $2.924840-0.6449974 .709016$ H $-2.740759-1.1659665 .660172$ C -2.1844200 .6989694 .767809$ H -1.1029300 .5695174 .873326$ H -2.3585131 .2843313 .857310$ H -2.5419561 .2992965 .614350$ C $-4.439803-0.4087354 .589844$ H -5.014145 - 1.3414234 .565105 H -4.8072600 .1846185 .436815$ H -4.678068 0.1469973 .675101 C $9.259421-0.809634-0.778592$ H $10.046889-0.560994-1.505721$ C $8.280946-1.786415-1.448101$ H $7.847333-1.375793-2.366459$ H $8.786661-2.724380-1.710707$ H $7.452349-2.035006-0.773026$ C $9.922236-1.4694580 .440094$ H $10.674043-0.8230150 .906134$ H $9.174620-1.7131811 .205567$ H 10.419182 -2.405744 0.156102 C $7.5963021 .623783-1.815953$ H $7.1191190 .777145-2.331166$ C $6.4835962 .630987-1.485875$ H $5.7439132 .214593-0.793504$ H $6.8896913 .538739-1.024922$ H $5.9607342 .936792-2.401728$ C $8.6229562 .231453-2.783678$ H $9.4182681 .523465-3.044948$ H $8.1384402 .544569-3.717148$ H $9.0959713 .120337-2.349556$ C 9.6261271 .9432890 .644794 H 9.7858111 .4323491 .605914 C $10.9944622 .080728-0.040636$ H $11.4734641 .109374-0.206209$ H $10.9096022 .577503-1.013469$ H 11.6733212 .6840040 .575489 C 9.0232403 .3228910 .946882 H 8.038949 3.2448471 .422503 H 9.6765413 .8968481 .616231 H 8.9047543 .9085010 .027550 222

2a_DAP-Ether-bridged

Si - 2.256115 -3.727134 2.322927 Si -7.794329 5.363685 -2.379292 Si $1.604894-0.0180395 .365581$ Si $9.327542-4.119552-2.561285$ O $0.241395-0.274524-0.010013$ C $-4.224789-1.7990901 .001793$ C - $3.534841-2.6699171 .501551$ C -6.976111 2.737466 -1.072139 C $-7.4472903 .757212-1.541641$ C $3.686726-1.1444143 .425482$ C $2.883461-0.7335194 .242961$ C $7.489726-3.109975-0.334124$ C $8.268873-3.529736-1.170266$ C $-0.346296-0.618325-1.260973$ C $1.1620320 .811204-0.041272$ C $1.0602861 .524678-3.106792$ C - $4.972699-0.7201880 .451627$ C $-4.3105280 .189977-0.397959$ N -3.024656 - $0.052422-0.734710$ C - $2.3993120 .831951-1.482547$ 
C $-1.0277560 .576528-1.865414$

C $-0.3665771 .536394-2.568403$

C $1.3709133 .049255-3.157391$

C $0.0312723 .793559-3.411465$

C $-0.0012165 .112052-2.624943$

C $-0.2268144 .099583-4.897376$

C $-0.9857062 .807429-2.869699$

C $-2.2909673 .049010-2.596199$

C $-3.0605872 .050264-1.912169$

$\mathrm{N}-4.3282412 .279680-1.627466$

C $-4.98686661 .358102-0.884190$

C $-6.3439061 .570340-0.562935$

C -7.0298510 .6425520 .258785$

C $-6.332555-0.5085500 .784543$

C $4.649111-1.6383512 .502519$

C $4.523517-1.3266841 .133229$

N $3.485382-0.5594850 .726537$

C $3.374857-0.293613-0.559632$

C $2.2621950 .512401-1.021093$

C $2.1370930 .740454-2.358883$

C $1.1227850 .853315-4.510881$

C $2.5759960 .360711-4.733431$

C $2.577877-0.949901-5.532001$

C $3.4621931 .386526-5.462841$

C $3.0523980 .149889-3.309906$

C $4.127903-0.570881-2.912623$

C $4.339345-0.809384-1.514865$

N $5.365465-1.544421-1.130990$

C $5.485489-1.8221670 .188841$

C $6.562669-2.6201410 .626921$

C $6.699186-2.9335602 .002063$

C $5.731166-2.4364172 .950667$

C $-0.632181-3.2119751 .480032$

C $0.643981-3.4608222 .294791$

C $-0.492586-3.8017230 .068651$

C $-2.291458-3.2457134 .164352$

C $-1.546961-4.2440905 .063159$

C $-3.719844-3.0352724 .687244$

C $-2.736524-5.5557352 .112396$

C $-3.409627-5.8594940 .765384$

C $-1.559574-6.5170512 .351330$

C $-9.2269345 .149955-3.613810$

C $-9.1465926 .170891-4.761192$

C $-9.3555073 .722810-4.166489$

C $-6.1676685 .839973-3.238828$

C $-5.0573706 .137054-2.220069$

C $-5.6973124 .760637-4.225718$

C $-8.2387916 .617923-1.027786$

C - $8.4594978 .028481-1.593630$

C $-9.4438676 .166907-0.190706$

C 2.4710860 .7897296 .859382

C 3.8038720 .0973637 .190330

C 2.6767882 .3061006 .749185

C $0.512656-1.4411115 .983815$

C $-0.738539-0.9183046 .704095$

C $1.273419-2.4453576 .858280$

C 0.5851931 .1441384 .259573

C -0.2236660 .3119893 .249168$

C 1.4493012 .1744913 .515456

C $9.358276-6.014757-2.485697$

C $7.950182-6.620706-2.576831$

C $10.081724-6.513375-1.226126$

C $11.066552-3.417686-2.270581$

C $12.085609-3.923391-3.302259$

C $11.073375-1.884343-2.200088$

C $8.570378-3.468464-4.182725$

C $8.991510-4.321266-5.391251$

C $7.042345-3.311185-4.135918$

C $8.988668-4.8486844 .298290$

C $9.102038-5.1365195 .621721$

C 8.142159-4.638658 6.564005

C $7.100482-3.8698526 .150959$

C $6.938224-3.5394434 .761915$

C $7.904599-4.0393303 .813600$

C $7.764592-3.7288322 .472274$

C $5.886539-2.7600994 .314265$

C - 10.4393730 .1282101 .772200 
C - $-11.074082-0.7552652 .586874$

C $-10.378830-1.8927813 .115539$

C $-9.072060-2.1088662 .811424$

C $-8.358311-1.2029231 .954113$

C $-9.057892-0.0574151 .422371$

C -8.3857260 .8281550 .598141$

C - $7.028049-1.3992361 .627582$

H $-1.079108-1.385936-1.023825$

H $0.398923-1.041441-1.943969$

H 1.5518120 .8802570 .971534

H $0.6549141 .751774-0.283518$

H $1.7684973 .351737-2.181571$

H $2.1233893 .303349-3.906054$

H -0.945199 5.644969-2.783118

H $0.8138045 .766368-2.954351$

H $0.1136574 .932342-1.550947$

H - $1.1764794 .634252-5.009633$

$\mathrm{H}-0.2868653 .195801-5.508293$

H $0.5713174 .734175-5.297974$

H - $2.7722473 .997343-2.817643$

H $0.450785-0.012847-4.506927$

H $0.7909271 .516446-5.311731$

H $2.120975-0.790881-6.515224$

H $2.011972-1.729005-5.010844$

H $3.597424-1.316922-5.692295$

H $3.0409321 .619701-6.446960$

H $4.4667560 .975270-5.609897$

H $3.5702302 .319596-4.904753$

H $4.812821-1.035864-3.615422$

H $-0.732050-2.1230031 .365498$

H $0.606999-2.9761423 .275620$

H $1.513821-3.0452331 .769384$

H $0.827403-4.5307312 .453651$

H $0.363654-3.350675-0.448876$

H - 1.385026 -3.624246 -0.542931

H $-0.322031-4.8838550 .098188$

H $-1.771183-2.2796384 .225101$

$\mathrm{H}-1.521475-3.8824416 .098975$

H - $0.511079-4.4101094 .748322$

H -2.054958 -5.215885 5.074100

H $-3.702652-2.7399425 .744458$

H - $4.309552-3.9586164 .616170$

$\mathrm{H}-4.248411-2.2580504 .126776$

H -3.480883 -5.741042 2.902644

H -3.737107 -6.906469 0.724975

H $-2.717740-5.701127-0.069959$

H -4.285034 -5.224413 0.594683

$\mathrm{H}-0.797750-6.4140631 .570711$

H - $1.905061-7.5585812 .331102$

$\mathrm{H}-1.066743-6.3518173 .314374$

H -10.142195 5.365520 -3.042825

H - $10.0333576 .099921-5.403628$

H -8.270059 $5.988342-5.394066$

H -9.081753 7.203139-4.399992

H -8.465190 3.430746-4.735764

H - $10.2168463 .646961-4.842678$

H $-9.4872602 .987930-3.365936$

H $-6.3729016 .760970-3.805169$

H -4.127319 6.416327 -2.734079

H -4.844963 5.249418 -1.612057

H -5.319478 6.959728 - 1.545392

H -5.496871 3.820103 -3.699423

H $-4.7666745 .070221-4.720530$

H -6.435915 4.562259-5.010313

H - $-7.3651976 .652864-0.361092$

H -7.603894 8.376400 -2.184336

H -8.620438 8.752322 - 0.784709

H $-9.3466198 .061381-2.238065$

H -9.637827 6.8742660 .625652

H -9.2817535 .1785400 .253685$

H - $10.3559126 .115143-0.798362$

H 1.7855020 .6120157 .702305

H $3.691883-0.9842267 .321735$

H 4.2299780 .5039688 .116461

H 4.5343240 .2557746 .388447

H 3.3753382 .5536425 .941259

H 3.1011692 .7042637 .679805 
H 1.7380852 .8373316 .557878 H $0.183050-1.9726905 .082343$ H - $0.474667-0.3495477 .604749$ $\mathrm{H}-1.380651-1.7494777 .023010$ H - $1.341848-0.2652396 .063378$ H $1.600891-1.9867327 .799368$ H $2.160423-2.8381976 .347795$ H 0.632793 -3.297788 7.117278 H -0.120052 1.6876734 .905487 H $0.427678-0.3346892 .648964$ $\mathrm{H}-0.7617130 .9609502 .547221$ H - $0.967534-0.3262503 .737876$ H 0.8369732 .7451782 .805033 H 2.2457501 .6817672 .945272 H 1.9221262 .8902814 .193209 H $9.930893-6.350908-3.362987$ H $7.993727-7.715968-2.520917$ H 7.448159-6.357847 -3.514130 H $7.317233-6.271411-1.752109$ H $10.085428-7.610192-1.188486$ H $9.581691-6.155829-0.316883$ H 11.123887 -6.177494 -1.188986 H $11.369199-3.796798-1.283528$ H $12.107962-5.017785-3.362380$ H $13.097769-3.584585-3.047378$ H $11.858957-3.537636-4.303646$ H $12.074371-1.510804-1.949360$ H $10.375435-1.509042-1.443647$ H $10.792957-1.439999-3.163048$ H $8.999069-2.464532-4.318725$ H $8.626987-3.874265-6.324818$ H $8.571375-5.332269-5.328769$ H $10.079021-4.422169-5.476221$ H $6.544022-4.281164-4.019966$ H $6.674850-2.866850-5.071012$ H $6.720286-2.678286-3.302680$ H $9.714069-5.2240753 .581833$ H $9.924347-5.7487515 .978794$ H $8.257355-4.8837387 .615265$ H $6.370949-3.4900336 .861055$ H $8.490780-4.1030461 .756350$ H $5.157671-2.3828655 .025742$ H - 10.9629800 .9912481 .370360 H -12.117805 -0.606618 2.845860 H $-10.910028-2.5823553 .764263$ H -8.541746 -2.969347 3.209868 H -8.908118 1.6937330 .200266 H -6.498539-2.2611372.023913 221

3a_DAP-Alkylene-bridged C $-1.8289820 .558182-4.614568$ C - $-2.357743-0.450551-3.785737$ $\mathrm{N}-1.732023-0.728284-2.618813$ $\mathrm{N}-4.039585-2.154369-3.384525$ C $-3.536331-1.171977-4.173254$ C $-4.185293-0.857498-5.383711$ C $-3.6719420 .167226-6.219373$ C $-2.4766970 .879130-5.834369$ C $-0.6316461 .226172-4.228645$ C $0.4083131 .792727-3.942147$ Si $2.0345652 .572230-3.536897$ C $3.4110711 .517047-4.319640$ C $4.7055972 .325852-4.506523$ C $3.0040990 .831231-5.632042$ C $2.1653752 .590556-1.644753$ C $2.1567221 .177103-1.048458$ C $3.3577113 .398209-1.114308$ C $2.0126954 .342015-4.220278$ C $1.7807954 .383789-5.737486$ C $0.9808065 .216362-3.494325$ C $-5.352532-1.581891-5.750577$ C $-6.352924-2.203650-6.058197$ Si $-7.846586-3.238519-6.371337$ C $-7.672677-4.748590-5.235421$ C $-7.430105-4.349881-3.772170$ C $-8.868593-5.704133-5.358301$ C $-7.822145-3.750887-8.197669$ 
C $-6.669919-4.719668-8.500423$ C $-7.764962-2.533778-9.132997$ C $-9.401015-2.228460-5.936702$ C $-9.228067-0.714226-6.125295$

C $-10.639095-2.731676-6.697507$ C $-0.517985-1.029974-0.102708$ C $-2.217707-1.689757-1.862588$ C $-1.535099-2.009312-0.622244$ C $-1.985598-3.0825980 .082766$ C - $1.398117-3.7606641 .308866$ C - $2.702790-4.1395142 .066091$ C $-3.775906-4.4654390 .985389$ C $-5.143425-3.9215261 .430519$ C $-3.928733-5.9672060 .689685$ C $-3.267593-3.684007-0.215993$ C $-3.943871-3.405460-1.356177$ C $-3.407359-2.428202-2.259605$ C 2.3011411 .8392354 .280032 C 2.0412630 .4583964 .176375 N 1.0756430 .0467893 .326328 N $2.574744-1.8221324 .804800$ C $2.794316-0.4909024 .943821$ C $3.791229-0.0375525 .831848$ C 4.0564751 .3511865 .952024 C 3.3068172 .3007915 .163097 C 1.5472332 .7368773 .472046 C 0.8933953 .4827212 .764904 Si -0.1615384 .4922301 .629320$ C - 1.3620645 .4513732 .744957 C - 2.5117666 .1434461 .996073 C -0.6282506 .4457113 .657326$ C - 1.0541193 .2111620 .541241 C -2.1735042 .4985461 .314090$ C - $1.5709963 .746724-0.801056$ C 1.0035735 .5859690 .598586 C $0.2991806 .779119-0.065572$ C 2.2483456 .0557731 .365331 C $4.531517-0.9934666 .580670$ C $5.160492-1.8265497 .207349$ Si $6.048390-3.2129668 .039176$ C $5.694522-3.0656559 .897828$ C 4.193046 -3.116002 10.212821 C $6.463285-4.10630910 .725280$ C $7.900594-2.9850457 .698261$ C $8.449051-1.7315248 .395671$ C $8.211376-2.9474626 .194823$ C $5.373523-4.8446267 .326082$ C $4.895133-4.7352605 .869731$ C $6.382221-5.9949967 .481884$ C $-0.798181-0.6737301 .370796$ C $0.856437-1.2440593 .202896$ C - $0.174094-1.6885552 .284824$ C $-0.381335-3.0267742 .165535$ C $-0.567742-5.0237630 .929856$ C $0.351673-5.3494632 .140850$ C $1.700244-5.8905831 .642025$ C $-0.246639-6.3715093 .123667$ C $0.519127-3.9800002 .776008$ C 1.463561 -3.605566 3.673581 C $1.644309-2.2102113 .952003$ C 5.0540131 .8347946 .823143 C 3.5981913 .6742615 .287868 C 4.5816964 .1336706 .145968 C 5.3307633 .1864796 .936236 C 6.3492503 .6820357 .820529 C 6.6018435 .0144977 .911172 C 5.8570675 .9553977 .126017 C 4.8845545 .5322166 .276199 C - $4.4436281 .847033-9.499895$ C $-3.9299732 .822435-10.295072$ C $-2.7387333 .524548-9.915082$ C - $2.1007383 .227911-8.752174$ C $-2.6061612 .204878-7.879158$ C $-3.8043301 .497988-8.261427$ C $-4.3031350 .507573-7.432963$ C $-1.9780661 .882568-6.688975$ H $3.6176250 .723769-3.586372$ 
H $5.5179311 .677679-4.858595$ H $4.5748903 .118802-5.252676$ H $5.0411132 .800210-3.577775$ H $3.8232790 .205208-6.008591$ H $2.1239380 .194410-5.499818$ H $2.7666041 .562683-6.413512$ H $1.2428043 .089396-1.315923$ H $3.1051940 .660996-1.241468$ H 2.0174451 .2107420 .040847 H $1.3536790 .565640-1.471245$ H $4.3086182 .927188-1.390457$ H $3.3704354 .426438-1.494991$ H $3.3294943 .452083-0.018173$ H $3.0113954 .757480-4.018747$ H $2.5571533 .844703-6.290443$ H $0.8138083 .936952-6.000124$ H $1.7737345 .419151-6.101391$ H $0.9869656 .239825-3.890392$ H - $0.0336254 .818967-3.624307$ H $1.1797495 .280081-2.418935$ H -6.776157 -5.279457 -5.586338 H -8.285539 -3.798720 -3.361040 H $-7.288755-5.241538-3.146946$ H $-6.541233-3.717702-3.668935$ H $-8.702952-6.614272-4.767994$ H $-9.787169-5.237112-4.981979$ H $-9.049999-6.010598-6.395504$ H - $8.768776-4.278838-8.385591$ H -6.657214 -4.987288 -9.564740 H $-5.699303-4.266037-8.263770$ H -6.753181 -5.649059-7.926699 H -7.763965 -2.850081 -10.183946 H $-8.618460-1.861804-8.991415$ H $-6.850834-1.951238-8.963324$ H $-9.573523-2.409486-4.865299$ $\mathrm{H}-10.138594-0.181143-5.822700$ H -8.394078 -0.324522 -5.533319 H $-9.033849-0.459658-7.174170$ H - $11.541496-2.210915-6.353205$ H - $10.545818-2.544935-7.774009$ $\mathrm{H}-10.805664-3.806159-6.563325$ H - $0.588302-0.128196-0.713006$ H $0.508745-1.399322-0.204924$ H -3.028907 -3.268547 2.646498 H - $2.571064-4.9654482 .765763$ H -5.448745 -4.399458 2.368429 H -5.102817 -2.839528 1.592731 H -5.914753 -4.126093 0.680268 H - $-4.713466-6.122581-0.058823$ H -3.014324 -6.421251 0.302578 H - $-4.218132-6.5029651 .600432$ H -4.915511 -3.833742 -1.584030 $\mathrm{H}-1.8070184 .6772133 .387894$ H -3.207602 6.605112 2.708318 H - -2.1467216 .9381741 .337111$ H -3.088072 5.4435331 .381971 H - 1.3195316 .8889274 .385360 H 0.1835435 .9659764 .216046 H -0.194151 7.269454 3.077934 H - 0.2788722 .4615610 .318187 $\mathrm{H}-1.8157542 .0831752 .262826$ H -3.000509 3.181976 1.539342 $\mathrm{H}-2.5847021 .6710860 .721930$ H -2.361431 $4.494331-0.663173$ H $-0.7758884 .211775-1.392910$ H - $1.9889762 .930274-1.403755$ H $1.3508654 .920946-0.203782$ H - $0.5928356 .482707-0.628224$ $\mathrm{H}-0.0077547 .5214310 .680214$ H $0.9778577 .283361-0.765385$ H 2.8069105 .2147011 .788315 H 2.9230106 .6083740 .698728 H 1.9810686 .7256002 .191390 H 6.060856 -2.068971 10.184189 H $4.013941-2.94655011 .282231$ H $3.634709-2.3569439 .654070$ H $3.766206-4.0952789 .963524$ 
H $6.318095-3.93389911 .799263$ H $6.108780-5.12180710 .510590$ H $7.541427-4.07960410 .528282$ H $8.405845-3.8626288 .128416$ H $9.518248-1.6046718 .183086$ H $7.935378-0.8277828 .043458$ H $8.331456-1.7804359 .483816$ H $7.911877-3.8714385 .688526$ H 7.688385 -2.116688 5.705819 H $9.286662-2.8097906 .023205$ H $4.495048-5.0874407 .942047$ H $5.726919-4.5082155 .191931$ H $4.456722-5.6866355 .538915$ H $4.146156-3.9469885 .741928$ H $6.716504-6.1238038 .517206$ H $5.937992-6.9444467 .157176$ H 7.274339 -5.825891 6.867058 H - 0.3678700 .3020801 .597747 H - $1.879074-0.5964981 .530227$ H $0.058757-4.7730580 .065800$ H - $1.185074-5.8768040 .646362$ H $2.196131-5.1682440 .985653$ H $2.373249-6.1115262 .477508$ H $1.546275-6.8186041 .079612$ H - $1.161240-6.0164713 .602818$ H $-0.471652-7.3096742 .604439$ H $0.474452-6.5879663 .919392$ H $2.154610-4.3101114 .126956$ H 5.6183041 .1211397 .416646 H 3.0311884 .3832044 .690787 H 6.9120002 .9662878 .413476 H 7.373523 5.379557 8.581841 H 6.0797077 .0139347 .217995 H 4.3181346 .2414535 .678945 H -5.345673 1.312420 -9.784781 H $-4.4194143 .079378-11.229413$ H - $2.3501824 .298893-10.569274$ H - $1.1977513 .757166-8.459894$ H -5.205426 - $0.025718-7.718187$ H - $1.0757052 .412108-6.398170$ 219

2a TAP-Unlinked

Si $1.743601-2.556261-2.217545$ Si -8.2847301 .1284090 .346811$ Si -2.130521 -1.879461 3.065545 Si 8.4115031 .1698480 .001034 C $-0.980460-1.496135-3.058248$ C $0.153885-1.915992-2.912546$ C $-6.2092880 .056871-1.588339$ C $-7.1785360 .413944-0.944506$ C $0.763184-1.0436103 .358060$ C $-0.412314-1.3372193 .482508$ C 6.0542190 .0985891 .598440 C 7.0860440 .4137631 .035634 C $0.0045955 .311536-0.321787$ C $-2.326405-1.045216-2.982897$ C - $2.6040380 .282608-2.596070$ $\mathrm{N}-1.5759271 .154768-2.476911$ C $-1.8233702 .348149-1.975865$ C $-0.7073833 .229577-1.690175$ C $-0.9866504 .388483-1.027937$ C -0.9170056 .2364410 .528494$ $\mathrm{H}-0.9563735 .8758171 .561212$ $\mathrm{H}-0.5357127 .2610630 .566178$ C $-2.3339996 .180170-0.096488$ C $-2.3380034 .818034-0.759057$ C $-3.4063274 .040591-1.052114$ H $-4.4277004 .322456-0.818696$ C $-3.1776782 .743208-1.618121$ $\mathrm{N}-4.1918641 .913689-1.758391$ C $-3.9337200 .663668-2.210254$ C $-4.975308-0.288343-2.199041$ C $-4.712502-1.605910-2.635069$ $\mathrm{N}-5.712126-2.513647-2.590761$ C $-5.421311-3.757600-2.930393$ C $-6.447415-4.761274-2.891533$ H -7.441932 -4.454960 -2.585075 
C $-6.155057-6.044043-3.228118$ H $-6.928594-6.804615-3.197281$ C $-4.827191-6.422157-3.628188$ $\mathrm{H}-4.633943-7.457953-3.888024$ C $-3.824641-5.507774-3.682728$ $\mathrm{H}-2.814813-5.770647-3.979600$ C $-4.082849-4.138901-3.335422$ $\mathrm{N}-3.097964-3.258517-3.383059$ C $-3.383151-1.983800-3.038338$ C $-2.4974917 .257087-1.186413$ $\mathrm{H}-1.7312467 .164215-1.963335$ H -3.476491 7.167559-1.668984 H $-2.4187678 .256552-0.744228$ C -3.428396 6.3399420.960624 H -3.319542 7.300877 1.475093 H -4.4257146 .3164940 .507443$ H -3.374622 5.5413541 .708734 C $0.6716902 .722130-2.002159$ H $0.6634102 .142538-2.927268$ H $1.0180692 .053481-1.208065$ H $1.4017663 .526660-2.093007$ C $2.118618-0.7227713 .078991$ C 2.4539610 .5492152 .568362 N 1.4625461 .4487862 .374792 C 1.7504682 .5635581 .733979 C 0.6591303 .4227721 .321922 C 0.9704954 .4700940 .507419 C $0.9496966 .093697-1.281673$ H $0.9787615 .604722-2.260269$ H $0.5951737 .114551-1.451066$ C $2.3658626 .083480-0.649740$ C 2.3336274 .8280020 .197914 C 3.3822254 .0798620 .615593 H 4.4123264 .3052770 .359115 C 3.1170112 .8724611 .342893 N 4.1026952 .0293991 .579779 C 3.7937240 .8339192 .135791 C $4.782833-0.1722432 .168162$ C $4.442748-1.4594502 .641707$ N $5.375640-2.4363232 .587221$ C $5.016551-3.6448252 .984966$ C $5.963448-4.7223722 .922135$ H $6.957316-4.4999292 .548818$ C $5.599620-5.9696143 .317194$ H $6.312747-6.7860753 .266503$ C $4.274447-6.2366263 .806055$ H 4.022717 -7.247372 4.110544 C $3.346061-5.2491443 .888613$ H $2.339538-5.4283094 .251919$ C $3.680115-3.9145683 .477415$ N $2.762010-2.9667113 .538068$ C $3.110277-1.7315143 .116042$ C 2.5611047 .2975810 .278925 H $2.5103888 .227091-0.299095$ H 1.7931097 .3359681 .058745 H 3.5376327 .2490270 .772286 C $3.4638236 .062543-1.715164$ H $3.3864215 .169591-2.345069$ H $3.3832716 .945412-2.358564$ H $4.4603646 .072858-1.259667$ C -0.7279112 .9888871 .699423$ $\mathrm{H}-0.7579742 .7127932 .755402$ H - 1.0221412 .0986821 .131607 H - 1.4755363 .7601561 .519794 C $2.790294-1.023743-1.815153$ H $2.688179-0.363577-2.688779$ C $4.286341-1.296689-1.605665$ H $4.750783-1.767963-2.479115$ H $4.821774-0.359356-1.408833$ H $4.467489-1.947699-0.742035$ C $2.209170-0.292911-0.601761$ H $1.136824-0.094490-0.707668$ H $2.343611-0.8905430 .306288$ H $2.7211470 .664925-0.445848$ C $2.619660-3.630913-3.505733$ H $3.586018-3.913986-3.062274$ C $1.859339-4.918751-3.850525$ 
H 2.358777 -5.456408 -4.666934 H $1.803960-5.598857-2.994678$ H $0.833754-4.703760-4.175363$ C $2.902533-2.809195-4.773676$ H $3.460483-3.405498-5.506696$ H $1.967291-2.490667-5.250236$ H $3.490361-1.908834-4.562062$ C $1.234297-3.456029-0.618111$ H $0.876034-2.6480260 .037782$ C $2.425334-4.1256230 .084121$ H $2.881749-4.900204-0.544789$ H $3.209090-3.4043570 .343533$ H $2.102925-4.6057921 .016762$ C $0.068243-4.440288-0.787360$ H - $0.259290-4.8125260 .192398$ H $-0.792872-3.971698-1.275906$ H $0.355007-5.312661-1.384441$ C - $8.323584-0.1463441 .750605$ H -7.273817 -0.266622 2.050909 C -9.1092590 .3448872 .974415$ H $-9.022339-0.3681863 .804215$ H -8.749772 1.3164113 .333711 $\mathrm{H}-10.1773440 .4465642 .745762$ C -8.827598 - 1.5173611 .280420 H -9.876518 - 1.4711470 .961647 H $-8.237810-1.8967490 .438581$ H $-8.766882-2.2530942 .092693$ C $-10.0219751 .450348-0.352963$ H $-10.5747790 .510486-0.206767$ C - 10.7587742 .5481200 .432451 $\mathrm{H}-10.2730463 .5227600 .304162$ H - 11.7922122 .6495340 .077693 H - 10.7971942 .3393031 .507248 C - $10.0290741 .758442-1.857608$ H -9.477877 $2.679159-2.082294$ H $-9.5703540 .951712-2.438439$ H -11.056457 1.894987 -2.219249 C -7.4159062 .7216290 .912892$ H -8.064358 3.1787231 .675627 C -7.247407 $3.722970-0.239364$ H -6.7475004 .6358100 .113626$ H -6.631117 3.290201-1.036238 H -8.206147 4.024121 -0.675595 C -6.0518162 .4227871 .554596$ H -5.386135 1.9275020 .837307 H -5.560059 3.352926 1.870230 H -6.138417 1.7792922 .437165 C $-2.414829-3.5816003 .851195$ H -3.437044 -3.889215 3.584121 C $-1.441865-4.6275173 .284947$ H - $1.582299-4.7753592 .208401$ H - $0.399004-4.3270413 .447335$ H - $1.587898-5.5994873 .773446$ C $-2.320880-3.5251065 .382822$ H -2.487852 -4.517761 5.820229 $\mathrm{H}-1.327548-3.1869415 .702935$ H -3.061883 -2.844853 5.816885 C $-3.384530-0.6191663 .724117$ H $-3.357320-0.7371364 .817537$ C $-4.813512-0.9320633 .254175$ H -5.539546 -0.290933 3.771263 H -4.921975 -0.742140 2.179610 H -5.101952 -1.973128 3.440642 C -3.0166800 .8370193 .410506$ H - 2.0026261 .0762423 .746733 $\mathrm{H}-3.0667611 .0396792 .334553$ H -3.712277 1.5284753 .903543 C - $2.020835-2.0433541 .178635$ $\mathrm{H}-1.174106-2.7358001 .062683$ C $-3.235668-2.6985750 .507833$ H - $2.998885-2.969816-0.528408$ H -3.549513 -3.615092 1.021014 H $-4.096581-2.0204640 .479217$ C - $1.622304-0.7368540 .480000$ H - $2.476794-0.0519120 .399845$ H $-0.818933-0.2168541 .014323$ H - $1.262737-0.939521-0.533041$ 
C $9.004362-0.085133-1.301790$ H $9.837271-0.625730-0.828374$ C $9.5566150 .617297-2.553540$ H $8.7609181 .146948-3.091216$ H $10.3366371 .349010-2.316040$ H $9.988899-0.114225-3.247915$ C $7.944244-1.123658-1.695549$ H $7.095551-0.651519-2.203280$ H $8.367580-1.864226-2.386495$ H $7.547600-1.656832-0.825538$ C $7.6097712 .702460-0.788601$ H $8.3379283 .096283-1.513474$ C $6.3164532 .357319-1.542937$ H $6.4764801 .606466-2.325345$ H $5.5610991 .969460-0.848925$ H $5.8976063 .251929-2.023394$ C 7.3312933 .7929250 .255933 H 8.2446564 .1368520 .753875 H $6.8657094 .669053-0.216761$ H 6.6405703 .4233961 .023568 C 9.8293901 .6712531 .156063 H 9.3854942 .3699931 .879627 C 10.3876140 .4782551 .944283 H $10.852879-0.2586611 .277904$ H 11.1584580 .8067082 .653111 H $9.604697-0.0345612 .513776$ C 10.9504962 .4100470 .410419 H 11.7110202 .7749271 .112327 H 11.4579281 .744687 -0.298921 H $10.5757353 .274041-0.151063$ 218

2b_TAP-Ether-bridged

Si $-2.937309-4.8394470 .753436$ Si -8.581681 $5.123552-2.192278$ Si $0.371953-1.4908955 .209668$ Si $10.107478-2.545895-1.419844$ O -0.006131 -0.379544 -0.137486 C $-4.465287-2.327115-0.102914$ C $-3.855145-3.3266280 .228508$ C - $7.2208652 .490027-1.434457$ C $-7.7731433 .537672-1.712686$ C $2.954074-1.7432633 .584076$ C $1.940935-1.6616974 .253158$ C $7.640456-2.2882650 .371539$ C $8.620767-2.413514-0.337956$ C $-0.371675-0.494077-1.507036$ C 0.8039390 .7412080 .197861 C $1.0908862 .041014-2.660182$ C -5.164536-1.145654-0.465373 C $-4.466644-0.046891-1.009730$ $\mathrm{N}-3.138881-0.170004-1.232901$ C - $-2.4892460 .874055-1.703789$ C - $1.0683340 .760700-1.955176$ C $-0.3937401 .875404-2.351482$ C $1.2868093 .560618-2.382016$ C $-0.0570304 .271869-2.690611$ C $-0.2853675 .426301-1.704803$ C $-0.1416644 .822504-4.125479$ C - $1.0581653 .153518-2.477794$ C $-2.3985753 .278701-2.327376$ C $-3.1728252 .132009-1.952533$ $\mathrm{N}-4.4745162 .255356-1.776136$ C $-5.1533701 .177901-1.316240$ C $-6.5471361 .280887-1.120464$ C - $7.2561440 .171215-0.605404$ C $-6.560730-1.043802-0.266370$ C $4.133052-1.8540672 .800546$ C $4.195012-1.2691541 .517647$ N $3.131388-0.5603871 .075311$ C $3.186782-0.045762-0.135884$ C $2.0630830 .726141-0.624933$ C $2.1140681 .200356-1.901324$ C $1.4164601 .654230-4.132280$ C $2.9277751 .310729-4.203941$ C $3.1638220 .157269-5.189323$ C $3.8065042 .503755-4.620584$ C $3.2213610 .888551-2.777591$ 
C $4.3150060 .222991-2.335499$ C $4.350019-0.250387-0.982969$ N $5.407043-0.913492-0.554400$ C $5.365410-1.4266390 .697780$ C $6.485333-2.1355341 .182517$ C $6.439269-2.6948862 .480418$ C $5.255333-2.5623203 .289256$ C $-3.863428-6.3677160 .095070$ C $-4.648002-6.101768-1.198254$ C $-2.923416-7.572188-0.078420$ C $-1.180638-4.7114760 .053049$ C $-0.383257-3.5939090 .738741$ C $-1.191186-4.515735-1.470339$ C $-2.911030-4.8154592 .650255$ C $-4.321622-4.8111863 .253971$ C $-2.061578-5.9546793 .230369$ C $-7.4639095 .919465-3.505091$ C $-7.9282837 .334210-3.882802$ C $-5.9679215 .901179-3.153994$ C $-8.8192596 .137651-0.603318$ C $-7.5004116 .678536-0.033382$ C $-9.8530747 .264367-0.755618$ C $-10.2741554 .667462-2.919009$ C $-10.1539013 .873483-4.227145$ C $-11.1119333 .891302-1.890337$ C $-0.466813-3.1920025 .186954$ C $0.404012-4.2818505 .825626$ C $-1.863948-3.1538305 .821231$ C $-0.674728-0.2092404 .284930$ C - $1.122807-0.7579602 .923819$ C 0.0786651 .1183664 .113745 C $0.788301-0.9466056 .985548$ C $-0.379695-0.1831437 .631792$ C $2.089576-0.1374707 .090664$

C $11.622320-2.438104-0.283025$ C $11.581496-3.5128150 .814292$ C $11.766618-1.0397860 .334705$ C $10.031039-1.068718-2.607577$ C $11.227807-1.036455-3.569326$ C $8.702821-1.011769-3.376388$ C $10.043262-4.193560-2.371730$ C $11.447939-4.669297-2.778597$ C $9.287798-5.307290-1.632074$ C $8.579064-4.5972524 .680345$ C $8.513831-5.1419635 .922407$ C $7.328134-5.0178726 .726176$ C $6.237471-4.3510936 .268250$ C $6.256376-3.7561574 .961150$ C $7.451456-3.8834844 .149835$ N $7.523191-3.3598112 .939021$ N 5.191617 -3.106456 4.525055 C - $10.650096-0.7012290 .266032$ C $-11.313152-1.7698970 .777960$ C $-10.616736-2.9783721 .126852$ C $-9.274560-3.0885350 .953573$ C $-8.527554-1.9869060 .414113$ C $-9.229567-0.7667930 .065084$ $\mathrm{N}-8.5924780 .279491-0.430308$ $\mathrm{N}-7.222408-2.1055840 .245418$ H - $1.046967-1.347206-1.548056$ H $0.501391-0.708627-2.133751$ H 1.0305590 .6172391 .255198 H 0.2547461 .6803960 .067331 H $1.5216963 .688061-1.318880$ H $2.1135943 .991101-2.949536$ H - $0.3025025 .064196-0.671629$ H -1.232958 $5.937980-1.904176$ H $0.5199256 .163458-1.798490$ H - $0.0610744 .038402-4.882010$ H $0.6569105 .552167-4.298465$ H - $1.1027885 .325705-4.276547$ H - $2.9122874 .231373-2.415466$ H $0.8346400 .761687-4.390156$ H $1.1435412 .434866-4.844351$ H $4.225794-0.103943-5.248249$ H $2.8336460 .448583-6.192759$

H $2.607373-0.736774-4.889543$ 
H $4.8565452 .195920-4.669682$ H $3.7431003 .338276-3.918363$ H $3.5098342 .863846-5.611734$ H $5.152468-0.023018-2.981509$ H - $4.594387-6.6271000 .874832$ H $-5.184208-7.005222-1.516622$ H -3.981784 -5.810141 -2.019131 H -5.381111 -5.299268 -1.069219 H -3.491955 -8.468529 -0.356883 H $-2.366343-7.8048860 .835889$ H $-2.189742-7.391785-0.873125$ H $-0.686621-5.6703130 .269780$ H $-0.860912-2.6173830 .606605$ H $0.626818-3.5153940 .316411$ H - $0.273602-3.7659711 .815797$ H $-0.169430-4.405882-1.856152$ H - $1.749805-3.612900-1.746254$ $\mathrm{H}-1.653680-5.361032-1.992295$ H -2.432093 -3.863440 2.920930 H $-4.277506-4.7052654 .345348$ $\mathrm{H}-4.850371-5.7481433 .039560$ H -4.928174 -3.987779 2.860907 H - $1.036151-5.9458092 .842671$ H -2.499835 -6.933515 2.999889 H - $2.000400-5.8792624 .323396$ H - $7.5932685 .279313-4.390300$ H -8.990998 7.364469-4.151531 $\mathrm{H}-7.3587677 .715740-4.739731$ H -7.774365 8.034616 -3.053091 H -5.623217 4.893789-2.896420 $\mathrm{H}-5.7463776 .555869-2.303910$ H -5.371801 6.258114-4.004166 H - -9.2217585 .4156580 .122488$ H -7.658282 7.119725 0.958960 H -7.086349 7.464512 -0.676083 H -6.742410 5.893306 0.065489 H - $10.8313026 .886109-1.071913$ H $-9.5324378 .011054-1.491138$ H -9.9944037 .7871100 .199015$ H - $10.7922875 .612296-3.140724$ H $-9.5829732 .948840-4.076361$ H -9.656481 $4.450308-5.014334$ H - $11.1458193 .590297-4.602000$ H -12.098670 3.641009-2.300427 H - $11.2715914 .464633-0.969945$ H - $10.6174772 .951236-1.614731$ H - $0.584015-3.4430334 .122887$ H $1.395768-4.3316115 .362565$ H -0.066258 -5.267708 5.719092 H $0.544346-4.1030816 .898846$ H - $1.808458-2.9144306 .890279$ H - $2.358687-4.1295265 .735982$ H $-2.515145-2.4123355 .343669$ H - $1.569162-0.0190494 .896125$ H - $0.266001-1.0609842 .312273$ H - $1.670561-0.0000942 .349961$ H - $1.779857-1.6293773 .026266$ $\mathrm{H}-0.5185131 .8323503 .531759$ H 1.0263060 .9660213 .582377 H 0.3122381 .5881635 .075807 H $0.932522-1.8767617 .554439$ H - $1.319073-0.7458627 .596558$ $\mathrm{H}-0.5527380 .7761617 .129624$ H - 0.1631910 .0342978 .685361 H 2.2959160 .1266718 .136000 H 2.0269570 .7985446 .522690 H $2.947466-0.6982256 .706218$ H $12.504535-2.625418-0.912832$ H $12.456969-3.4312731 .471336$ H $11.574529-4.5264230 .398501$ H $10.685262-3.4031931 .437100$ H $11.901771-0.265208-0.428145$ H $12.634355-0.9994591 .005524$ H $10.881104-0.7765590 .926597$ H $10.083587-0.174829-1.969540$ H $12.186885-1.063648-3.038390$ H $11.212977-0.125351-4.180834$ 
H $11.203023-1.889295-4.258893$ H $8.659831-0.116820-4.010694$ H $7.843522-0.987432-2.697184$ H $8.585330-1.881643-4.034885$ H $9.486717-3.974850-3.295128$ H $12.044706-4.940302-1.899492$ H 12.005660 -3.905792 -3.332043 H $11.383631-5.560000-3.416248$ H $8.260547-5.012805-1.395765$ H $9.779828-5.567457-0.687401$ H $9.248925-6.218250-2.243245$ H $9.463224-4.6791614 .056994$ H $9.364530-5.6822486 .325095$ H $7.316068-5.4681847 .713487$ H $5.333158-4.2449686 .857962$ H - $11.1561230 .219744-0.003596$ H -12.386531 -1.722042 0.930769 H -11.183164 -3.809687 1.534061 H -8.728888 -3.990552 1.209400 217

2c TAP-Alkylene-bridged

C - $1.905717-0.459185-4.813600$ C $-2.534165-1.206483-3.796930$ $\mathrm{N}-1.966977-1.229074-2.569679$ $\mathrm{N}-4.351795-2.644037-3.074554$ C $-3.752867-1.922304-4.053204$ C $-4.349635-1.870131-5.330191$ C $-3.736319-1.104624-6.349981$ C $-2.506990-0.399426-6.093132$ C $-0.6762010 .208703-4.563798$ C $0.3929200 .760256-4.375870$ Si $2.0547101 .562683-4.278796$ C $2.4281981 .915409-2.452785$ C $3.8276122 .511122-2.241546$ C $2.1857540 .724523-1.514315$ C $1.9309553 .172596-5.278368$ C $1.4490722 .885641-6.709630$ C $1.0392874 .222411-4.602244$ C $3.2651600 .378669-5.142939$ C $4.5726671 .061070-5.574288$ C $3.555325-0.886982-4.324237$ C $-5.559669-2.569458-5.576650$ C $-6.599344-3.176393-5.751467$ Si $-8.209500-4.061901-5.886567$ C $-7.937469-5.748508-6.726659$ C $-6.529444-6.324170-6.516414$ C $-9.008643-6.769882-6.309083$ C $-9.342828-2.958207-6.932122$ C $-10.735847-3.575350-7.123532$ C $-8.706654-2.598348-8.282726$ C $-8.858774-4.243938-4.111988$ C $-7.840169-4.955085-3.206952$ C $-9.252254-2.885900-3.513136$ C $-0.878769-0.9431410 .007238$ C $-2.551368-1.931350-1.622886$ C $-1.943058-1.961703-0.305974$ C $-2.509653-2.7779690 .625177$ C $-2.033351-3.1464622 .020695$ C $-3.394058-3.2173882 .769642$ C $-4.446568-3.7395331 .747217$ C $-5.777792-2.9960921 .946827$ C $-4.719485-5.2500041 .847102$ C $-3.824418-3.3464710 .416765$ C $-4.429421-3.335187-0.795265$ C $-3.782027-2.664986-1.885015$ C 2.1325952 .7138253 .458466 C 1.7423481 .3901193 .747276 $\mathrm{N} 0.7314730 .8524163 .031810$ N 2.061879 -0.665041 4.997199 C 2.4062080 .6252004 .765960 C 3.4455071 .2090365 .520892 C 3.8337602 .5425345 .246465 C 3.1824693 .2957274 .204924 C 1.4738163 .4414472 .430002 C 0.8972744 .0524681 .548472 Si 0.0302035 .1174550 .312690 C - 1.4071535 .9433361 .233357 
C -0.9129966 .9252692 .304632$ C -2.3524124 .8982191 .845483$ C $-0.5780334 .085716-1.164799$ C $-0.8424202 .608406-0.853682$ C $-1.7922284 .719326-1.862252$ C $1.3109106 .387167-0.276349$ C $2.6101325 .714460-0.741384$ C $0.7478997 .304919-1.371506$ C 4.0980960 .4642156 .538253 C $4.647996-0.1814717 .410705$ Si $5.424324-1.1695198 .760704$ C $4.065752-2.3034359 .447196$ C $2.926630-1.51587710 .109315$ C $3.515141-3.2255158 .347715$ C 6.0054570 .06818110 .077857 C 6.7543671 .2841119 .511403 C $6.812913-0.60811511 .196078$ C $6.795251-2.2216507 .971223$ C $8.006319-1.3925337 .521266$ C $7.236732-3.3995868 .854046$ C $-1.216586-0.2034031 .320363$ C $0.389183-0.3952133 .268776$ C $-0.681141-0.9818302 .487007$ C $-0.986642-2.2878032 .709751$ C - $1.300850-4.5222492 .029788$ C $-0.449978-4.5819743 .328950$ C $0.851918-5.3532343 .065542$ C $-1.170542-5.2381814 .520402$ C $-0.173373-3.1101733 .578078$ C $0.792103-2.5840244 .371354$ C $1.093744-1.1868554 .266060$ N 4.8315903 .0895205 .975667 N 3.5562244 .5631513 .922312 C 4.5356965 .0846074 .640157 C 5.1867134 .3308315 .693618 C 6.2418804 .9532956 .442933 C 6.6159166 .2272966 .158215 C 5.9718476 .9739585 .112106 C 4.9687806 .4283214 .377217

C $-4.325592-0.243031-9.806869$ C $-3.7222660 .492108-10.776374$ C -2.492009 $1.190412-10.521232$ C $-1.8956031 .136704-9.302390$ C $-2.4943560 .373105-8.243975$ C $-3.734303-0.332298-8.501352$ $\mathrm{N}-4.327717-1.051319-7.564011$ $\mathrm{N}-1.9080730 .329185-7.060291$ H $1.6960282 .688444-2.180833$ H $3.9554782 .839172-1.202339$ H $4.6080091 .769133-2.449274$ H $4.0115893 .379098-2.886256$ H $1.2120830 .258401-1.696208$ H $2.952296-0.047843-1.638642$ H $2.2127941 .048653-0.465364$ H $2.9489853 .585091-5.338304$ H $2.1155102 .192480-7.236045$ H $0.4466142 .440465-6.704796$ H $1.4033043 .811714-7.296955$ H $1.4688874 .566970-3.655317$ H $0.9169085 .103920-5.244583$ H $0.0376823 .825474-4.392914$ H $2.7362940 .068153-6.055991$ H $5.2205890 .349459-6.101961$ H $4.3943221 .905848-6.248388$ H $5.1364021 .436987-4.712568$ H 2.636489 -1.372968 -3.977102 H $4.115455-1.616393-4.922990$ H $4.164796-0.653916-3.443233$ H -8.060014 -5.561273 -7.803827 H $-6.328331-6.515822-5.455726$ H $-6.419054-7.277687-7.048921$ H -5.754262 -5.641632 -6.878458 H $-8.900729-7.698476-6.883694$ H -8.915480 -7.030450 -5.248045 H - $10.027060-6.398657-6.468468$ H $-9.459249-2.026100-6.360853$ H - $10.682030-4.493637-7.721384$ 
$\mathrm{H}-11.211964-3.826020-6.168037$ H $-11.400982-2.882154-7.653903$ H -7.731575 -2.114725 -8.155137 H $-8.558407-3.490427-8.904295$ H $-9.353068-1.913690-8.846696$ H $-9.763241-4.867672-4.167389$ H -6.900755 -4.390832 -3.156909 H $-8.229798-5.049452-2.184641$ H -7.604288 -5.964087 -3.563261 H - $10.051220-2.399799-4.083787$ H $-9.607362-3.005315-2.481412$ H $-8.393605-2.203459-3.488207$ H $-0.843123-0.232205-0.819295$ H $0.119679-1.3888570 .079587$ H -3.667979 -2.203674 3.084378 H $-3.360885-3.8382593 .665399$ H -6.533479 -3.336796 1.230987 H -6.160906 -3.181048 2.956866 H -5.648721 -1.916336 1.819871 H -5.094435 -5.499186 2.845803 $\mathrm{H}-5.482151-5.5408941 .116517$ H -3.833450 -5.857946 1.653672 H $-5.423973-3.738239-0.961426$ H - -1.9711226 .5135480 .480245$ H - -0.2833996 .4171633 .045548$ $\mathrm{H}-0.3259917 .7426531 .871715$ H - 1.7590387 .3728932 .841743 $\mathrm{H}-1.8274014 .2787272 .582581$ H -3.192067 5.3850392 .357838 H - 2.7718084 .2263421 .088299 H $0.2543534 .124087-1.879811$ H - $1.0587552 .050673-1.774794$ H $0.0198652 .136834-0.372228$ H - $1.7013802 .487931-0.182275$ H - $2.0363214 .166477-2.778419$ H -2.680187 4.694127 -1.219546 H - $1.6170065 .763437-2.143229$ H 1.5516237 .0063700 .599389 H 2.4334095 .047546 -1.594961 H $3.3450526 .463319-1.063272$ H 3.0631735 .1160420 .056086 H $1.4714268 .086569-1.635350$ H $0.5339366 .740092-2.287724$ H -0.178888 7.802032 -1.061092 H $4.537241-2.93210510 .217359$ H $3.274366-0.93201910 .968390$ H $2.461711-0.8225369 .397494$ H $2.142307-2.19498510 .467513$ H $3.053789-2.6396737 .543211$ H $4.297426-3.8483227 .898987$ H $2.747560-3.8977668 .752104$ H 5.0722010 .44445410 .522347 H 6.9504892 .01652910 .305264 H 7.722481 0.9982269 .085849 H 6.1800211 .7836618 .723990 H $7.784454-0.95675810 .825714$ H 7.011056 0.096055 12.014039 H $6.289139-1.47197811 .622424$ H $6.328266-2.6421497 .068305$ H $8.563654-1.0093518 .384278$ H $8.700144-2.0063656 .932909$ H $7.712060-0.5353096 .905231$ H $6.399899-4.0566319 .114905$ H $7.985380-4.0098908 .332880$ H $7.692004-3.0552689 .789646$ $\mathrm{H}-0.7565570 .7840591 .321477$ H -2.300007 -0.059190 1.391385 H $-0.628406-4.5563451 .164598$ H - $1.979156-5.3720151 .947541$ H $1.433898-4.8835112 .266104$ H $1.476951-5.3969353 .963818$ H $0.622298-6.3821212 .766036$ H - $-0.491311-5.2974095 .377750$ H -2.052306 -4.681336 4.843738 H - $1.481107-6.2563364 .261235$ H 1.417668 -3.193073 5.016682 H 6.7153054 .3723317 .227494 
H 7.4133716 .6993816 .722978 H 6.3006187 .9893414 .915563 H 4.4684886 .9733403 .583796 H -5.254050 -0.777435 -9.978479 H $-4.1674360 .560450-11.763759$ H -2.041645 $1.766899-11.322869$ H - $0.9675101 .656204-9.087667$ 201

3a PTD-Unlinked

Si $1.450933-1.9979323 .426053$ Si $-0.700701-1.886026-7.516347$ Si -4.107123 -2.558556-1.631055 Si -1.494377 2.061471 8.578475 C 2.264413 -1.975178 0.487158 C $2.100680-1.9853631 .694124$ C $1.123729-2.057475-5.088742$ C $0.530696-2.055312-6.151608$ C $-4.300944-1.6203161 .267898$ C $-4.334849-2.0728100 .137990$ C -3.0900400 .7328046 .353100$ C - 2.5817191 .2211207 .345224 C $2.268766-1.955053-0.931648$ C $1.895861-0.785878-1.626254$ N $1.6944610 .337964-0.900277$ C $1.1847551 .390024-1.500518$ C $0.7861882 .514263-0.679332$ C $0.0871403 .507185-1.293631$ C $-1.5838715 .172766-1.806669$ H -2.584372 $4.729193-1.829344$ H - $1.7190516 .253272-1.702129$ C $-0.8281124 .819428-3.113974$ C - $1.7865474 .630106-4.290863$ $\mathrm{H}-2.3592725 .547913-4.462532$ H - $1.2433854 .399414-5.213781$ H -2.493189 $3.815400-4.097897$ C $0.2131825 .903688-3.452822$ H $0.7925655 .615855-4.336397$ H - $0.2879096 .855478-3.661425$ H $0.9157756 .059888-2.627247$ C $-0.0948343 .551147-2.726551$ C $0.3136942 .541989-3.531639$ H $0.1461092 .533684-4.603909$ C $0.9067761 .381117-2.928960$ N $1.1350260 .309974-3.659840$ C $1.594975-0.805269-3.038072$ C $1.660146-2.003168-3.778615$ C $2.103079-3.159822-3.102224$ N $2.214728-4.388368-3.642779$ S $2.665586-5.371111-2.447474$ N $2.738169-4.348374-1.204082$ C 2.407416 -3.136936 -1.689934 C -4.148911 -1.018642 2.542873 C -3.624774 0.286784 2.644142 $\mathrm{N}-3.3326070 .9389291 .494711$ C -2.7669462 .1219941 .567415$ C -2.3166792 .7407030 .336821$ C - 1.6187273 .9041290 .451950 C 0.0397895 .6408910 .181375 H 1.0445065 .2635770 .396259 H $0.1655616 .566236-0.388388$ C - 0.7189305 .8923531 .510343 C 0.2360346 .2375082 .654449 H 0.8106097 .1369912 .408235 H -0.3105236 .4358573 .583007$ H 0.9408295 .4198282 .841437 C - 1.7611257 .0147431 .339622 $\mathrm{H}-1.2604537 .9625641 .112802$ $\mathrm{H}-2.4616336 .7938230 .527160$ H -2.342632 7.140908 2.258982 C - 1.4530244 .5829241 .717064 C -1.8848734 .0451962 .882411$ $\mathrm{H}-1.7329394 .5200843 .846452$ C - 2.5088632 .7525242 .853991 $\mathrm{N}-2.8025222 .1418773 .983249$ C -3.3335760 .8940643 .920766$ C -3.5372420 .1878805 .124441$ C - $-4.071149-1.1148485 .026846$ 
N $-4.305766-1.9441416 .062022$ S -4.863968 -3.316462 5.427576 $\mathrm{N}-4.843974-2.9717363 .853257$ C -4.382535 -1.711052 3.748016 C $-0.7669074 .577954-0.621224$ C $0.003814-3.2272773 .419591$ $\mathrm{H}-0.378655-3.2510944 .451393$ C $-1.127848-2.7417392 .503186$ $\mathrm{H}-0.795469-2.6972391 .458994$ H - $1.990586-3.4189232 .546252$ H - $1.482467-1.7426752 .778358$ C $0.432935-4.6501863 .035670$ H $1.160655-5.0668513 .740357$ $\mathrm{H}-0.433249-5.3241363 .018135$ H $0.886332-4.6679492 .037001$ C $0.802471-0.2473853 .766936$

H $0.130774-0.0226802 .924393$ C $-0.021159-0.1669655 .058267$ H $-0.840333-0.8948075 .081638$ H $0.606176-0.3460915 .939840$ H -0.4674170 .8271075 .176762$ C 1.9169100 .8064173 .773285 H 2.5385480 .7506542 .873057 H 1.4939131 .8182883 .827929 H 2.5737050 .6800014 .642858 C $2.825205-2.4909544 .641274$ H $3.363181-1.5598044 .871910$ C $3.843935-3.4746204 .046605$ H $4.637468-3.6937234 .772622$ H $3.374035-4.4278453 .777981$ H $4.313975-3.0746913 .142326$ C $2.247932-3.0340025 .959505$ H $1.543867-2.3374356 .427984$ H $1.716994-3.9802085 .800605$ H $3.051072-3.2279086 .681631$ C $0.052146-0.890525-8.948972$ H $-0.1268130 .164328-8.692600$ C $1.568971-1.077942-9.098303$ H $2.101904-0.811747-8.179832$ H $1.823934-2.117121-9.337736$ H $1.957572-0.450056-9.910404$ C $-0.666833-1.174672-10.278229$ $\mathrm{H}-1.749162-1.019079-10.210124$ H $-0.286932-0.517632-11.070667$ $\mathrm{H}-0.502883-2.208357-10.605317$ C - $1.221365-3.635233-8.029035$ H $-1.926598-3.522715-8.865751$ C $-1.948226-4.360374-6.885946$ $\mathrm{H}-2.863010-3.839483-6.581256$ H $-2.231579-5.376239-7.189510$ H - $1.304130-4.447703-6.002011$ C $-0.026496-4.465784-8.520638$ H $0.456993-4.017027-9.395269$ Н $0.731996-4.565125-7.734590$ H $-0.345336-5.477459-8.802412$ C - $2.159404-0.970673-6.715899$ H $-2.491531-1.639588-5.910129$ C $-3.335053-0.784229-7.684659$ H -3.654315 - $1.730298-8.137709$ $\mathrm{H}-4.202617-0.357107-7.165515$ H -3.072485 -0.095126 -8.496840 C $-1.7591690 .359900-6.064272$ $\mathrm{H}-0.9420860 .230943-5.346028$ $\mathrm{H}-1.4330611 .090458-6.816157$ $\mathrm{H}-2.6135490 .800709-5.532644$ C $-4.536324-1.060413-2.722171$ H $-3.607081-0.472886-2.776041$ C $-5.626631-0.149579-2.140923$ $\mathrm{H}-5.3768170 .189494-1.130653$ H -5.768758 $0.735929-2.774027$ H -6.591074 -0.668110 -2.082373 C $-4.898891-1.492781-4.152337$ H $-4.152801-2.164611-4.590474$ $\mathrm{H}-5.862251-2.016203-4.174571$ H $-4.984377-0.619617-4.811681$ C -5.197346 -4.062807-1.995204 H -5.086015 -4.275996 -3.068788 
C $-4.724011-5.297083-1.213296$ H $-3.699215-5.581426-1.476298$ H $-4.753428-5.115373-0.131620$ H -5.370881 -6.159216 -1.419835 C $-6.680732-3.777687-1.718104$ H $-7.064078-2.955117-2.331695$ H -7.293704 -4.662687 - 1.931441 H -6.840617 -3.510712 -0.666329 C - $2.267922-2.998906-1.780950$ $\mathrm{H}-2.111674-3.814022-1.059253$ C $-1.882059-3.514111-3.175277$ H -2.513968 -4.347363 -3.504795 H - $1.958676-2.717186-3.925414$ H $-0.840444-3.861026-3.191502$ C $-1.355105-1.837958-1.366715$ H $-1.650415-1.394208-0.409387$ H $-0.323444-2.189403-1.263430$ $\mathrm{H}-1.356147-1.042471-2.124003$ C 0.0591350 .9776848 .734852 H 0.5895571 .1410807 .785598 C $-0.224767-0.5276118 .834598$ H - $0.857807-0.8734068 .010715$ H $0.713168-1.0978718 .802444$ H - $0.730033-0.7826719 .772880$ C 0.9845321 .4446279 .868472 H 1.2091832 .5160719 .806883 H 0.5352001 .25390710 .850297 H 1.9389320 .9039029 .837628 C -2.4759072 .30964110 .182302$ H -3.4455462 .7103059 .851012$ C -1.8459343 .34625911 .125710$ H - 1.7060824 .31725810 .637999 H - 2.4859983 .50612412 .002684 H -0.8677333 .01601511 .492921$ C -2.7478520 .99064510 .919974$ H -3.191212 0.234872 10.261659 H - 1.8227760 .57198111 .333860 H -3.436850 1.149531 11.759181 C -1.0504353 .7411567 .808465$ H -0.3844474 .2460168 .524811$ C -0.3025463 .5981596 .474217$ H 0.6355483 .0415026 .576784 $\mathrm{H}-0.9259553 .0784375 .736603$ H - -0.0544594 .5863466 .063129$ C - 2.3013504 .6126357 .618276 H -2.0325155 .5939537 .206255$ H -2.997726 4.139350 6.915349 H - 2.8387894 .7844658 .557717 C - $2.5374401 .963137-0.930713$ H $-3.5678071 .602683-0.976685$ H -2.331198 2.547402 -1.826045 H - $1.8932641 .076134-0.952151$ C 1.0352812 .3918830 .796140 H 2.0708212 .0932500 .974573 H 0.8419093 .3166901 .337176 H 0.4032951 .6082351 .229930 200

3b PTD-Ether-bridged

Si - 1.927368 -4.507010 3.590580

Si -6.297517 -4.685116 -7.435042

Si - 4.6783721 .7935183 .753898

Si 6.6802554 .9372903 .618243

O - $-1.017191-0.3538500 .199103$

C $-3.181290-4.5977470 .794816$

C $-2.724249-4.5312961 .921081$

C $-5.343364-4.797379-4.530560$

C $-5.778571-4.795769-5.666911$

C -1.8044712 .8559673 .839881$

C -2.9527272 .4558123 .785223$

C 3.7021704 .4916234 .087975

C 4.8902914 .7495644 .033123

C $-0.159339-1.270782-0.470680$

H - $0.298416-2.2176850 .047879$ H $0.890996-0.973616-0.378000$ C - $1.0984430 .946972-0.375377$ $\mathrm{H}-1.7317831 .5156170 .304101$

H - $1.5780470 .917261-1.359565$ 
C $0.9140240 .564045-2.786269$ C $-3.708908-4.666262-0.519491$ C $-3.186155-3.868474-1.558201$ $\mathrm{N}-2.142854-3.056093-1.268464$ C $-1.675672-2.274943-2.216951$ C $-0.575493-1.384802-1.909808$ C $-0.167175-0.509829-2.868769$ C $0.4259991 .563372-3.876703$ H -0.269416 2.270980 -3.410529 H $1.2404612 .146675-4.310034$ C $-0.3341160 .747953-4.955941$ C - $1.4975441 .571868-5.523827$ H -2.052616 $1.007410-6.280685$ H - $1.1147252 .482200-5.998559$ H - $2.1965211 .862426-4.732810$ C $0.5633890 .288999-6.119906$ H $-0.036343-0.246550-6.863690$ H $1.359298-0.386020-5.796379$ H $1.0215481 .154867-6.610237$ C $-0.825040-0.442536-4.156508$ C $-1.808330-1.306974-4.503426$ H $-2.319885-1.260572-5.460173$ C $-2.266901-2.270895-3.544902$ $\mathrm{N}-3.256695-3.082065-3.858535$ C $-3.740266-3.902259-2.892801$ C $-4.807394-4.765550-3.218925$ C $-5.316911-5.580122-2.185895$ $\mathrm{N}-6.329116-6.460563-2.309637$ S - $6.535683-7.120946-0.853757$ $\mathrm{N}-5.399614-6.3660840 .004203$ C $-4.778361-5.526243-0.846403$ C -0.4588193 .2986363 .901078$ C 0.4302423 .0787802 .828934 $\mathrm{N}-0.0482302 .4613911 .723479$ C 0.7785142 .2221730 .729746 C $0.2796451 .546734-0.450980$ C $1.1601591 .264599-1.451562$ C $2.328986-0.008470-3.092278$ H $2.410792-0.996760-2.624969$ H $2.509243-0.139003-4.160600$ C $3.3707070 .943575-2.446956$ C $4.5658590 .141509-1.913480$ H $5.3081650 .798101-1.446794$ H $5.057711-0.389484-2.736103$ H $4.245559-0.595151-1.169552$ C $3.8908242 .024465-3.411843$ H $4.6442312 .641777-2.910416$ H $3.1001932 .693696-3.759634$ H $4.3584641 .558957-4.286292$ C $2.5707721 .565629-1.320132$ C $3.0632842 .238205-0.253175$ H $4.1199172 .457794-0.130915$ C 2.1773472 .6086840 .811318 N 2.6574253 .2290421 .868832 C 1.8147803 .4839602 .899226 C 2.3326234 .1302274 .042261 C 1.4411844 .3642145 .109478 N 1.7525964 .9634126 .275423 S 0.4015084 .9748287 .154093 $\mathrm{N}-0.6350064 .2472286 .157316$ C 0.0581453 .9498745 .040962 C $-0.265269-3.6143403 .382998$ H $0.263053-3.6966024 .344842$ C $0.590853-4.3034832 .309671$ H $1.557956-3.7972632 .196207$ H $0.087506-4.2807851 .335062$ H $0.791068-5.3533992 .553725$ C - $0.448179-2.1236173 .063085$ H $0.517294-1.6504142 .840776$ H - $0.894745-1.5752323 .899785$ H - $1.093301-1.9700962 .190896$ C - $3.056613-3.5390764 .768629$ H $-2.861127-2.4907824 .507771$ C - $2.661989-3.7117076 .242604$ H $-3.229672-3.0169226 .874686$ H - $1.596393-3.5172286 .412145$ H - $2.878200-4.7262956 .597000$ 
C $-4.555950-3.7879234 .560011$ H -5.148431 -3.149805 5.229621 H - $4.829216-4.8268484 .776202$ H $-4.861655-3.5698233 .531110$ C $-1.652664-6.3299264 .047892$ H - $1.209445-6.7732173 .143960$ C $-0.651966-6.5254695 .196836$ H - $-0.480111-7.5940925 .378512$ H - $1.022156-6.0914586 .132265$ H $0.319265-6.0671464 .978928$ C - $2.969594-7.0687274 .325567$ H -3.439493 -6.707210 5.247987 H -2.793807 -8.144806 4.449595 H -3.689564 -6.939134 3.509430 C $-6.517926-6.457081-8.075582$ H -6.928050 -6.373292 -9.092830 C $-5.175520-7.196911-8.164673$ H $-5.321820-8.227696-8.511945$ H $-4.684536-7.246943-7.184717$ H $-4.484313-6.708794-8.860341$ C $-7.519526-7.248888-7.221670$ H - $7.628316-8.273370-7.600010$ H $-8.514421-6.790210-7.220634$ H -7.184165 -7.313987-6.179353 C $-4.872211-3.803096-8.326612$ H $-4.004622-4.471325-8.228679$ C $-5.160505-3.614018-9.823200$ H $-4.287539-3.194429-10.338977$ H -5.993956 - $2.917766-9.977835$ H -5.416531 -4.557647 - 10.319499 C $-4.504645-2.468017-7.661929$ H -3.642370 -2.011255 -8.165743 H $-4.249828-2.599611-6.604389$ H $-5.332409-1.750181-7.723273$ C $-7.907342-3.675612-7.552680$ H $-7.585990-2.627555-7.644727$ C $-8.706902-4.027999-8.818284$ H $-9.566144-3.354850-8.930185$ H $-9.098795-5.050872-8.767475$ H $-8.105730-3.951485-9.730941$ C $-8.797134-3.776661-6.305344$ H $-9.687398-3.144205-6.415824$ $\mathrm{H}-8.267041-3.460001-5.401634$ H $-9.142925-4.803904-6.139754$ C -5.8720183 .2380723 .391042$ H -6.7853463 .0085073 .960834$ C -5.3312404 .5884423 .888841$ H -6.0854965 .3752353 .760154$ $\mathrm{H}-4.4411004 .8823153 .320932$ H -5.048335 4.565629 4.945913 C -6.2599313 .3465581 .909003$ H -6.949861 4.185618 1.752551 H -6.7526442 .4407581 .540281$ $\mathrm{H}-5.3793133 .5263021 .279490$ C -5.028189 1.094442 5.485517 H -6.0646230 .7252495 .452096$ C - 4.9509642 .1801266 .569497 H -5.137214 1.7470307 .560671 H -5.693250 2.9697936 .411438 H -3.958877 2.648138 6.594196 C $-4.115984-0.0883915 .838143$ H - $4.300202-0.4291316 .865428$ $\mathrm{H}-3.0563320 .1860695 .764116$ H -4.286950 - 0.9412735 .175220 C -4.7791990 .4670382 .396628$ H - 4.8582611 .0298861 .455203 C -6.055176 - 0.3765122 .549027 H -6.162323 -1.0738411 .708748$ $\mathrm{H}-6.9623030 .2389762 .588757$ H -6.022803 -0.9770403 .466685$ C $-3.540322-0.4296632 .285189$ H -3.603252 -1.083596 1.406522 H -3.439981 -1.078689 3.160318 H -2.614495 0.1446002 .193535 C 6.8574664 .2195101 .867169 H 7.904160 4.381567 1.568503 C 5.9497844 .9335490 .853799 
H $6.0943254 .516876-0.152295$ H 6.1504426 .0091930 .796686 H 4.8955614 .7969291 .121662 C 6.5766802 .7095821 .845454 H 6.6873552 .3108250 .827629 H 5.5495092 .5047432 .170369 H 7.2596242 .1489472 .493202 C 7.6605863 .9105614 .876462 H 7.3134332 .8752004 .750012 C 9.1697633 .9435754 .593463 H 9.7088033 .2758145 .277281 H 9.5766894 .9518904 .738806 H 9.4069223 .6314593 .569388 C 7.3625184 .3281366 .323165 H 7.8908623 .6782957 .032320 H 6.2920974 .2718396 .549108 H 7.6921915.356063 6.519362 C 7.1784556 .7719383 .702624 H 7.5278326 .9326264 .733257 C 8.3550297 .0835992 .762467 H 8.7061928 .1123492 .912260 H 8.0589556 .9900061 .710980 H 9.2085416 .4159312 .923608 C 6.0125547 .7400683 .455350 H 6.3482158 .7809293 .548594 H 5.1975287 .5812854 .168519 H 5.5961397 .6183592 .448283 199

3c PTD-Alkylene-bridged

C $\overline{2} .344604-0.255201-4.545230$ C $2.3436740 .620381-3.441041$ N $1.2885570 .569125-2.594472$ N $3.4004002 .368941-2.124681$ C $3.4265901 .544722-3.201860$ C $4.5381891 .606488-4.068388$ C $4.5384180 .737458-5.180108$ N $5.5040950 .661794-6.116057$ S $5.032668-0.468429-7.164579$ N $3.624176-0.926617-6.528257$ C $3.450904-0.184097-5.418243$ C $1.268824-1.152520-4.768374$ C $0.330922-1.905542-4.956980$ Si $-1.164023-2.936180-5.312225$ C $-2.545945-2.317442-4.164701$ H $-3.469320-2.826733-4.479066$ C $-2.764011-0.802100-4.295735$ H -3.021812 -0.503050 -5.317637 H $-3.579230-0.472328-3.638982$ $\mathrm{H}-1.860622-0.252953-4.002997$ C $-2.266979-2.679610-2.699903$ H $-2.224993-3.760562-2.529611$ H - $1.307276-2.261064-2.381666$ H $-3.039316-2.270989-2.035404$ C - $0.721236-4.738002-4.910197$ H $-0.276687-4.691903-3.904627$ C -1.946018 -5.662151 -4.832211 H -2.691115 -5.308206 -4.111381 H $-2.443202-5.755037-5.803742$ H $-1.644522-6.671699-4.525035$ C $0.337832-5.316088-5.858742$ H $1.215243-4.665136-5.942270$ H $0.680558-6.297843-5.508277$ H $-0.072335-5.456817-6.865635$ C - $1.621009-2.598805-7.125929$ H - $2.025242-1.575633-7.110903$ C $-0.427853-2.599014-8.093064$ H $0.370410-1.928263-7.758683$ H $0.003312-3.600517-8.197909$ H $-0.746108-2.275198-9.092320$ C $-2.734898-3.529475-7.630073$ H $-3.592938-3.563359-6.948495$ H -3.103720 -3.198483 -8.609005 H $-2.365209-4.554241-7.753412$ C $5.5992452 .507476-3.803077$ C $6.4716243 .299931-3.499587$ Si $7.5996964 .573653-2.781423$ C $6.8383365 .014137-1.089104$ 
H 7.615937 $5.560866-0.534210$ C $5.6089765 .926800-1.224566$ H $5.8468606 .892622-1.680160$ H $4.8323355 .447470-1.833143$ H $5.1726196 .128742-0.237199$ C $6.4401433 .770270-0.275778$ H 7.274745 3.084999-0.105643 H 6.0530714 .0703240 .707677 H $5.6478413 .214619-0.790687$ C $7.6866376 .042511-3.987284$ H $8.3464165 .686818-4.792823$ C $8.3698877 .260120-3.343637$ H $9.3531207 .011691-2.927810$ H $8.5182598 .056986-4.083107$ H 7.764001 7.678741 -2.531537 C $6.3528616 .431823-4.639681$ H $5.8756945 .574000-5.124860$ H $5.6446906 .833819-3.907615$ H $6.5106787 .206419-5.401029$ C $9.3577113 .854755-2.667120$ H $10.0171494 .729901-2.561773$ C $9.6103092 .939174-1.462893$ H $9.4575413 .460138-0.511985$ H $8.9481592 .064950-1.480569$ H $10.6428442 .566901-1.473325$ C $9.7397823 .137408-3.972106$ H 9.620079 3.779114 -4.852077 H $10.7865372 .809939-3.941388$ H $9.1163382 .248467-4.124470$ C $-0.8284280 .177421-0.785695$ $\mathrm{H}-0.445411-0.490309-1.558110$ H - $1.8223910 .502139-1.113528$ C $1.2681091 .377888-1.559479$ C $0.1314941 .328586-0.657593$ C 0.1069472 .2218660 .367395 C -0.9799002 .4775841 .395553$ C -0.1084992 .8102852 .642194$ H 0.1838331 .8668933 .118034 H -0.6356263 .4022663 .391139$ C 1.1670473 .5358772 .125465 C 2.3858583 .0987142 .953852 H 2.2435763 .3780864 .003975 H 2.5294042 .0146072 .902343 H 3.3023423 .5813632 .597621 C 1.0781295 .0712722 .170965 H 0.8975745 .4090513 .197364 H 2.0239905 .5079711 .832001 H 0.2877845 .4731341 .533744 C 1.2744663 .0102350 .704598 C $2.3485963 .093056-0.115743$ H 3.2384703 .6647580 .130692 C $2.3641812 .306187-1.314368$ C -3.975204-4.188347 2.033650 C $-4.053853-2.7925412 .210902$ $\mathrm{N}-3.023225-2.0390491 .760758$ $\mathrm{N}-5.241031-0.8212522 .992922$ C $-5.191142-2.1688062 .844814$ C $-6.278152-2.9369263 .312489$ C $-6.198936-4.3357583 .144750$ $\mathrm{N}-7.130895-5.2263823 .535273$ S -6.568829 -6.680838 3.125784 N $-5.157506-6.2982422 .447196$ C $-5.057215-4.9569272 .512861$ C $-2.846946-4.7848131 .414346$ C $-1.865006-5.2846110 .896400$ Si $-0.307316-6.0405180 .244113$ C $1.004715-4.6667030 .220774$ H $1.962903-5.149039-0.024276$ C $1.145671-3.9856081 .590884$ H $1.417650-4.6914822 .383316$ H $1.922178-3.2106421 .557415$ H $0.205994-3.5000571 .881768$ C $0.701563-3.615825-0.855515$ H $0.712288-4.029937-1.869227$ H - $0.288266-3.179053-0.691310$ H $1.430644-2.795673-0.826773$ C $-0.665281-6.649652-1.518010$ 
H -1.141619 -5.789719 -2.012521 C $0.604081-6.994461-2.311750$ H $1.307345-6.155916-2.359780$ H $1.134399-7.846673-1.873358$ H $0.347704-7.268492-3.343071$ C $-1.663150-7.815238-1.553130$ H - $2.571090-7.597585-0.979688$ H - $1.963450-8.040191-2.584317$ H - $1.216069-8.726954-1.139895$ C $0.184978-7.3930671 .485270$ H $0.533522-6.8310072 .364540$ C $-0.977055-8.2835001 .949776$ H - $1.819576-7.6940112 .326080$ H - $1.350894-8.9126631 .134726$ H $-0.646825-8.9539592 .753570$ C $1.361436-8.2450230 .984074$ H $2.199315-7.6341360 .628118$ H $1.741188-8.8917131 .784995$ H $1.050724-8.8987420 .160586$ C $-7.393178-2.3016873 .913487$ C $-8.315190-1.6796434 .407544$ Si $-9.530416-0.4683815 .090744$ C -8.8448011 .2462814 .613698$ H -9.6669011 .9612734 .769981$ C -7.6626031 .6673135 .501253$ H -7.9380641 .7636036 .555678$ H -6.843407 0.9412485 .430233 H -7.267637 2.638929 5.175608 C -8.405121 1.3207373.141148 H -9.206486 1.0677152 .441934 H -8.064525 2.337010 2.899842 H - 7.5689860 .6362142 .956986 C $-9.659052-0.7461596 .968365$ H - $10.274074-1.6537847 .061576$ C -10.4256320 .3956627 .655593$ $\mathrm{H}-11.4063840 .5702287 .198402$ H - 10.5937910 .1666398 .715357 H -9.8679501 .3384597 .611113$ C $-8.328784-1.0315057 .679079$ H -7.791865 -1.863205 7.211165 H -7.665737 - 0.1601867 .665081 H -8.504599 - 1.2913828 .730796 C - $11.250314-0.8350184 .364094$ H $-11.958273-0.3282345 .037740$ C $-11.489525-0.2915042 .950005$ $\mathrm{H}-11.3931110 .7983832 .907420$ H -10.781517 -0.722688 2.231795 H $-12.499997-0.5473322 .606019$ C - $11.554619-2.3411114 .415297$ H $-11.443151-2.7558355 .423284$ H $-12.582888-2.5393834 .087838$ H $-10.880441-2.8970943 .753120$ C $-0.943303-0.5819430 .553484$ H - $1.266797-1.6072400 .370991$ H $0.041345-0.6417191 .030737$ C $-3.076788-0.7358321 .917255$ C -1.9666100 .0703131 .442538$ C -2.0203291 .4087911 .676503$ C - 1.9047173 .6688441 .008570 H -2.160624 $3.573598-0.053178$ $\mathrm{H}-1.4324794 .6425861 .142871$ C -3.200961 3.533487 1.858233 C -4.4190883 .9516511 .019094$ $\mathrm{H}-4.3233565 .0012450 .718693$ H -4.5032273 .3408560 .114442$ H -5.3488403 .8472961 .588712$ C -3.1951214 .3689853 .150252$ H -3.063633 5.430453 2.913224 $\mathrm{H}-4.1522424 .2540953 .670803$ H - 2.4084684 .0733583 .847369 C -3.2377102 .0417102 .140971$ C -4.2897121 .3258932 .604084$ H -5.215714 1.7842072 .938628 C - $4.227575-0.1056922 .551130$ 


\section{References}

1. Fulmer, G. R.; Miller, A. J. M.; Sherden, N. H.; Gottlieb, H. E.; Nudelman, A.; Stoltz, B. M.; Bercaw, J. E.; Goldberg, K. I., NMR Chemical Shifts of Trace Impurities: Common Laboratory Solvents, Organics, and Gases in Deuterated Solvents Relevant to the Organometallic Chemist. Organometallics 2010, 29 (9), 2176-2179.

2. $\quad$ Zhang, J.; Kang, H.; Martin, J.; Zhang, S.; Thomas, S.; Merkel, T. C.; Jin, J., The Enhancement of Chain Rigidity and Gas Transport Performance of Polymers of Intrinsic Microporosity via Intramolecular Locking of the Spiro-Carbon. Chem. Commun. (Camb.) 2016, 52 (39), 6553-6.

3. Lindner, B. D.; Engelhart, J. U.; Tverskoy, O.; Appleton, A. L.; Rominger, F.; Peters, A.; Himmel, H. J.; Bunz, U. H., Stable Hexacenes Through Nitrogen Substitution. Angew. Chem. Int. Ed. 2011, 50 (37), 8588-91.

4. An, C.; Zhou, S.; Baumgarten, M., Condensed Derivatives of Thiadiazoloquinoxaline as Strong Acceptors. Cryst. Growth Des. 2015, 15 (4), 1934-1938.

5. Appleton, A. L.; Brombosz, S. M.; Barlow, S.; Sears, J. S.; Bredas, J.-L.; Marder, S. R.; Bunz, U. H. F., Effects of Electronegative Substitution on the Optical and Electronic Properties of Acenes and Diazaacenes. Nat. Commun. 2010, 1, 91.

6. Miao, S.; Appleton Anthony , L.; Berger, N.; Barlow, S.; Marder Seth , R.; Hardcastle Kenneth, I.; Bunz Uwe, H. F., 6,13-Diethynyl-5,7,12,14-tetraazapentacene. Chem. Eur. J. 2009, 15 (20), 4990-4993.

7. Ahrens, L.; Butscher, J.; Brosius, V.; Rominger, F.; Freudenberg, J.; Vaynzof, Y.; Bunz, U. H. F., Azaacene Dimers: Acceptor Materials with a Twist. Chem. Eur. J. 2020, 26 (2), 412-418.

8. Cardona, C. M.; Li, W.; Kaifer, A. E.; Stockdale, D.; Bazan, G. C., Electrochemical Considerations for Determining Absolute Frontier Orbital Energy Levels of Conjugated Polymers for Solar Cell Applications. Adv. Mater. 2011, 23 (20), 2367-71.

9. Wollscheid, N.; Perez Lustres, J. L.; Kefer, O.; Hahn, S.; Brosius, V.; Bunz, U. H. F.; Motzkus, M.; Buckup, T., Oxygen-Catalysed Sequential Singlet Fission. Nat. Commun. 2019, $10(1), 5202$. 